\title{
Feldfrüchte für die Biogaserzeugung - Index der relativen Anbauwürdigkeit (IrA)
}

\author{
Dissertation \\ zur Erlangung des Doktorgrades (Dr. sc. agr.) \\ der Fakultät für Agrarwissenschaften \\ der Georg-August-Universität Göttingen
}

\author{
vorgelegt von \\ Katharina Hey \\ geboren am 19. Juni 1981 in Heiligenstadt
}

Göttingen, im August 2020 
1. Gutachter: Prof. Dr. Rolf Rauber

2. Gutachter: Prof. Dr. Klaus Dittert

Tag der mündlichen Prüfung: 2. Oktober 2020 


\section{Inhaltsverzeichnis}

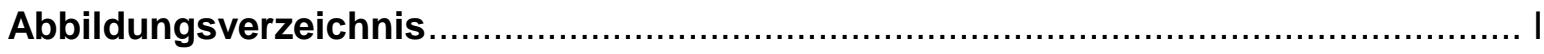

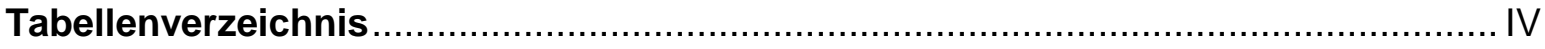

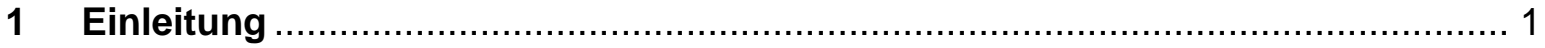

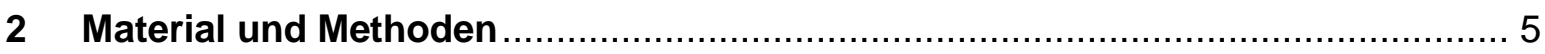

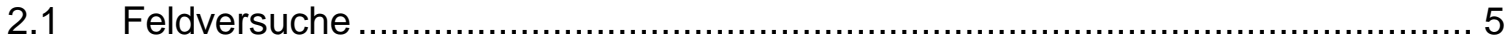

2.1.1 Standorteigenschaften und Witterung .............................................. 5

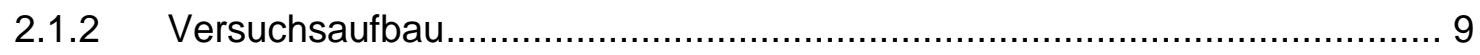

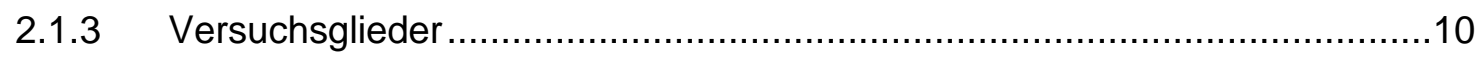

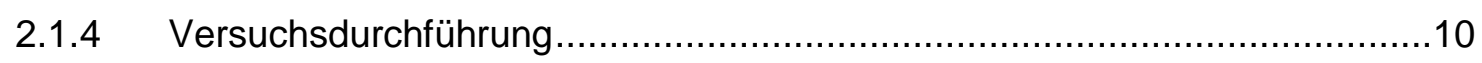

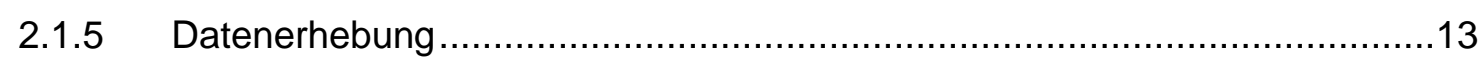

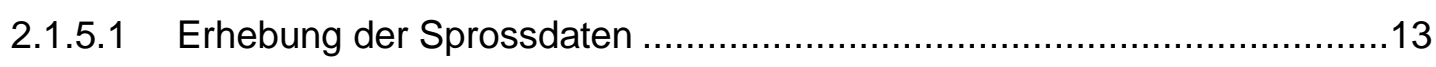

2.1.5.2 Erhebung der Wurzeldaten ....................................................... 14

2.1.5.3 Erhebung der Bodendaten ............................................................ 15

2.2 Umfrage zur Erhebung der Akzeptanz des Energiepflanzenanbaus in der

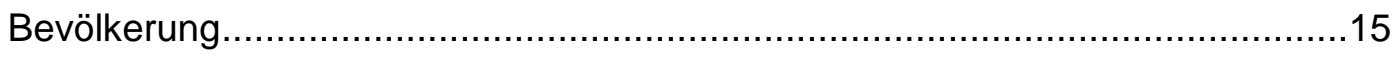

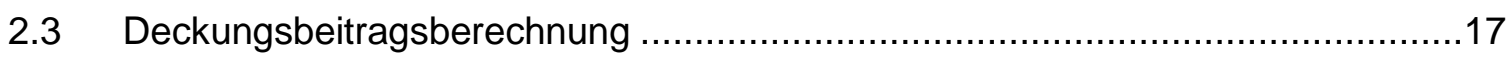

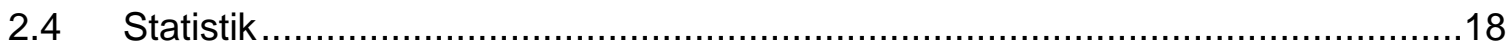

2.5 Berechnung des Index der relativen Anbauwürdigkeit (IrA) .............................18

2.5.1 Berechnung der Teilindizes des Index IrA .............................................18

2.5.2 Berechnung des Gesamtindex IrA ...................................................21

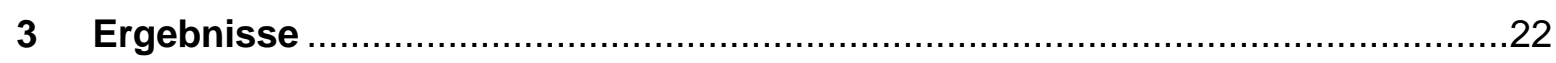

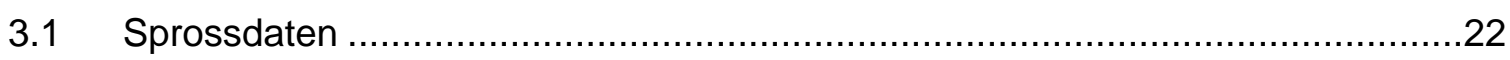

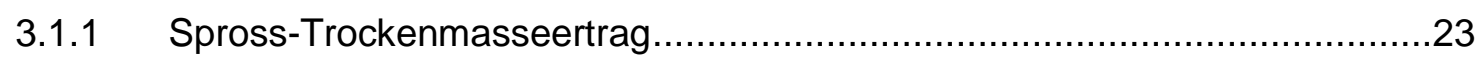

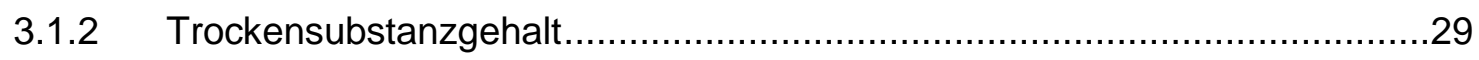

3.1.3 Stickstoff in der Spross-Trockenmasse ..................................................

3.1.4 Spezifischer Methanertrag und Methanflächenertrag...............................34

3.1.5 Spurenelementgehalte Cobalt und Nickel .............................................

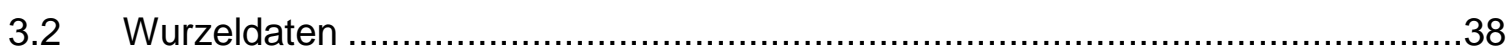

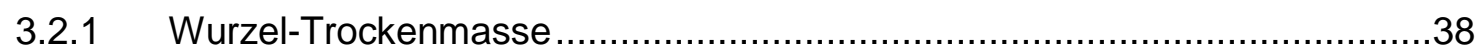

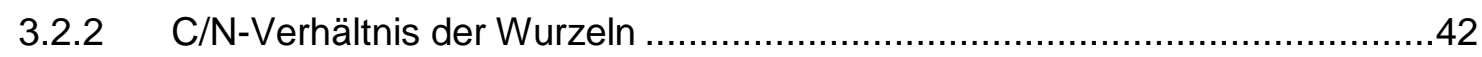

3.2.3 Stickstoff in der Wurzel-Trockenmasse ..................................................4

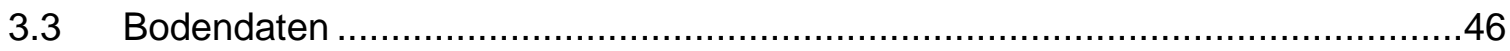

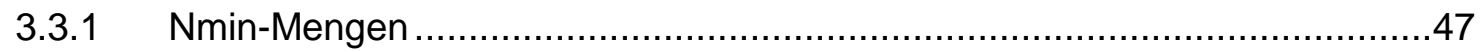

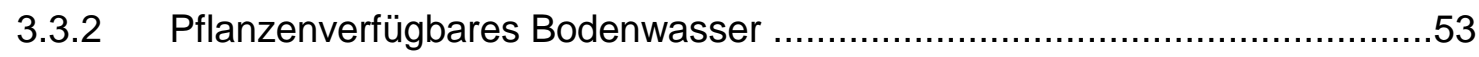

3.4 Akzeptanz des Energiepflanzenanbaus in der Bevölkerung .............................56 


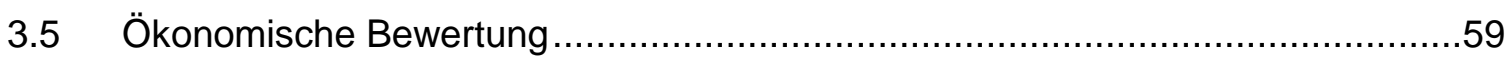

3.6 Zusammenhänge zwischen ausgewählten Parametern der

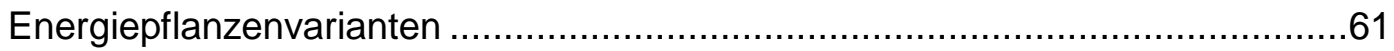

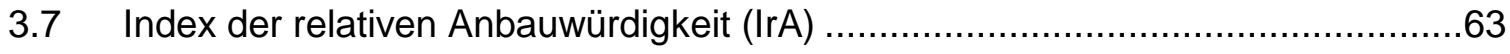

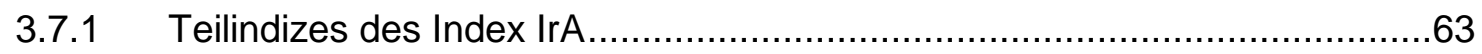

3.7.2 Teilindex Soziale Aspekte (Akzeptanz) ................................................69

3.7.3 Gesamtindex IrA an den Standorten Reinshof und Schoningen..................69

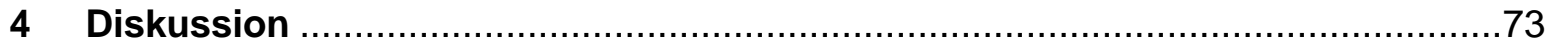

4.1 Teilindizes des Index der relativen Anbauwürdigkeit (IrA) …..........................73

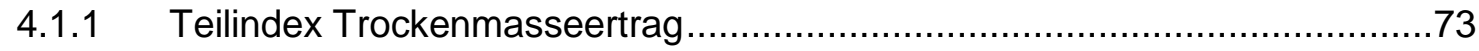

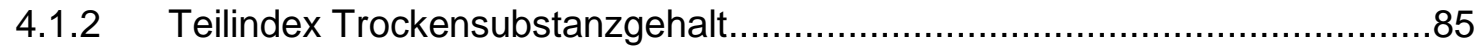

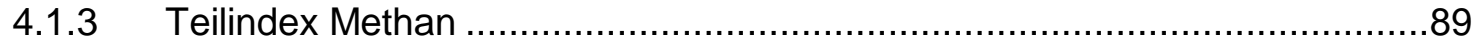

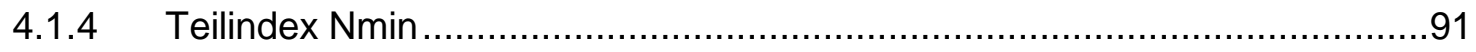

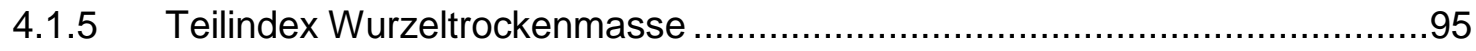

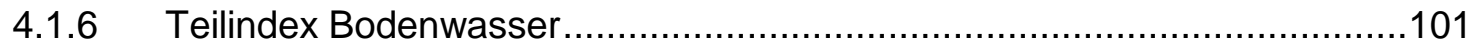

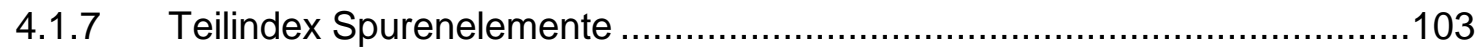

4.1.8 Teilindex Ökonomische Aspekte ......................................................110

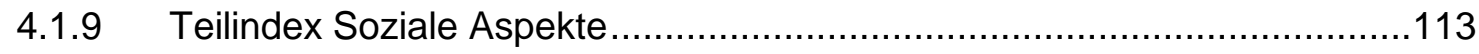

4.2 Gesamtindex der relativen Anbauwürdigkeit - IrA .....................................116

4.2.1 Bewertung der Varianten mit dem Gesamtindex IrA ................................116

4.2.2 Bewertung des Index IrA ............................................................ 119

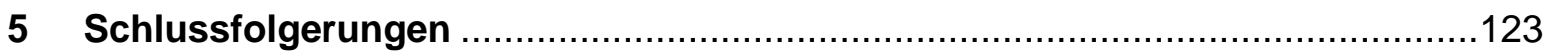

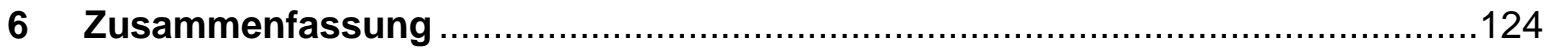

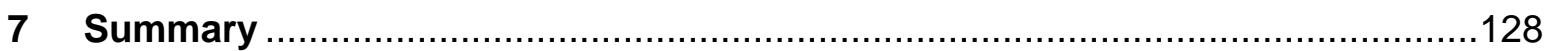

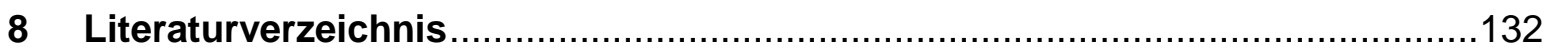

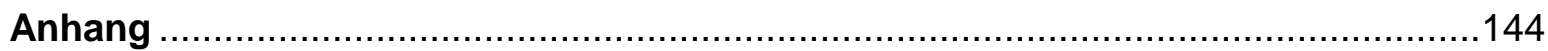




\section{Abbildungsverzeichnis}

Abbildung 1: Beziehung zwischen Wassergehalt und Wasserspannung für den Boden am Standort Reinshof $(A)$ und den Boden am Standort Schoningen (B). Unterteilung in die Bodenabschnitte $0-30 \mathrm{~cm}, 30-60 \mathrm{~cm}$ und $60-80 \mathrm{~cm}$.

Abbildung 2: Monatliche Niederschlagssummen im Versuchszeitraum und im langjährigen Mittel (1981-2010). Standort Reinshof: Wetterstation Gartemühle, Standort Schoningen: Wetterstation Uslar (Deutscher Wetterdienst)....

Abbildung 3: Monatliche Mittelwerte der Lufttemperatur im Versuchszeitraum und im langjährigen Mittel (1981-2010). Standort Reinshof: Wetterstation Gartemühle, Standort Schoningen: Wetterstation Wahlsburg-Lippoldsberg (Deutscher Wetterdienst).

Abbildung 4: Trockenmasseerträge in den Versuchsjahren 2015 und 2016 am Standort Reinshof. Dargestellt sind die Mittelwerte \pm Standardabweichung. Varianten mit gleichen Großbuchstaben (2015) bzw. gleichen Kleinbuchstaben (2016) sind nicht signifikant verschieden, $p<0,05$ (Tukey-Test), * kennzeichnen signifikante Unterschiede zwischen den Versuchsjahren innerhalb einer Variante, $p<0,05$ (t-Test). Bei gestapelten Säulen beziehen sich die Angaben auf die Höhe der Gesamtsäule. n.v. = Wert nicht verfügbar. Keine Angabe der Standardabweichung bei Mais als Zweitfrucht im Versuchsjahr 2016 aufgrund geschätzter Werte (Abschnitt 2.1.5.1) .23

Abbildung 5: Pflanzenbestände des Gemenges aus Amarant und Mais a) am Standort Reinshof im Versuchsjahr 2015 am 23. Juli 2015 (Hauptversuch mit Amarantsorte Bärnkrafft) und b) am Standort Reinshof im Versuchsjahr 2016 am 16. Juli 2016 (Amarant-SortenZusatzversuch mit Amarantsorte Pastewny)....

Abbildung 6: Pflanzenbestände des Gemenges aus Winterackerbohnen und Wintertriticale als Erstfrucht im Zweikulturnutzungssystem mit Mais a) am Standort Reinshof im Versuchsjahr 2015 (Aufnahmedatum 13. April 2015), b) am Standort Reinshof im Versuchsjahr 2016 (Aufnahmedatum 6. April 2016), c) am Standort Schoningen im Versuchsjahr 2015 (Aufnahmedatum 7. April 2015) und d) am Standort Schoningen im Versuchsjahr 2016 (Aufnahmedatum 13. April 2016). .25

Abbildung 7: Trockenmasseerträge in den Versuchsjahren 2015 und 2016 am Standort Schoningen. Dargestellt sind die Mittelwerte \pm Standardabweichung. Varianten mit gleichen Großbuchstaben (2015) bzw. gleichen Kleinbuchstaben (2016) sind nicht signifikant verschieden, $p<0,05$ (Tukey-Test). Bei gestapelten Säulen beziehen sich die Angaben auf die Höhe der Gesamtsäule. n.v. = Werte nicht verfügbar. .26

Abbildung 8: Pflanzenbestände der einjährigen Blühmischung a) am Standort Reinshof im Versuchsjahr 2015 am 23. Juli 2015 und b) am Standort Reinshof im Versuchsjahr 2016 am 21. Juli 2016. Pflanzenbestände der mehrjährigen Blühmischung c) am Standort Reinshof im Versuchsjahr 2015 am 23. Juli 2015 und d) am Standort Reinshof im Versuchsjahr 2016 am 10. Juli 2016. Pflanzenbestände der einjährigen Blühmischung e) am Standort Schoningen im Versuchsjahr 2015 am 28. Juli 2015 und f) am Standort Schoningen im Versuchsjahr 2016 am 18. Juli 2016. Pflanzenbestände der mehrjährigen Blühmischung g) am Standort Schoningen im Versuchsjahr 2015 am 28. Juli 2015 und h) am Standort Schoningen im Versuchsjahr 2016 am 18. Juli 2016. .28

Abbildung 9: Pflanzenbestände der Durchwachsenen Silphie a) am Standort Reinshof im Versuchsjahr 2016 am 30. August 2016 und b) am Standort Schoningen im Versuchsjahr 2016 am 18. Juli 2016. 
Abbildung 10: Stickstoffakkumulation in der oberirdischen Biomasse in den Versuchsjahren 2015 und 2016 am Standort Reinshof. Dargestellt sind die Mittelwerte \pm Standardabweichung. Varianten mit gleichen Großbuchstaben bzw. gleichen Kleinbuchstaben sind nicht signifikant verschieden, $p<0,05$ (Tukey-Test). Bei gestapelten Säulen beziehen sich die Angaben auf die Höhe der Gesamtsäule. n.v. = Wert nicht verfügbar. .32

Abbildung 11: Stickstoffakkumulation in der oberirdischen Biomasse in den Versuchsjahren 2015 und 2016 am Standort Schoningen. Dargestellt sind die Mittelwerte \pm Standardabweichung. Varianten mit gleichen Großbuchstaben bzw. gleichen Kleinbuchstaben sind nicht signifikant verschieden, $p<0,05$ (Tukey-Test). Bei gestapelten Säulen beziehen sich die Angaben auf die Höhe der Gesamtsäule. n.v. = Werte nicht verfügbar.

Abbildung 12: Trockenmasseerträge der Wurzeln in einer Tiefe von $0-60 \mathrm{~cm}$ in den Versuchsjahren 2015 und 2016 am Standort Reinshof. Dargestellt sind die Mittelwerte \pm Standardabweichung. Varianten mit gleichen Großbuchstaben bzw. gleichen Kleinbuchstaben sind nicht signifikant verschieden, $p<0,05$ (Tukey-Test). Bei gestapelten Säulen beziehen sich die Angaben auf die Höhe der Gesamtsäule. n.v. = Werte nicht verfügbar.

Abbildung 13: Trockenmasseerträge der Wurzeln in einer Tiefe von $0-30 \mathrm{~cm}$ in den Versuchsjahren 2015 und 2016 am Standort Schoningen. Dargestellt sind die Mittelwerte \pm Standardabweichung. Varianten mit gleichen Großbuchstaben bzw. gleichen Kleinbuchstaben sind nicht signifikant verschieden, $p<0,05$ (Tukey-Test). Bei gestapelten Säulen beziehen sich die Angaben auf die Höhe der Gesamtsäule. n.v. = Werte nicht verfügbar.

Abbildung 14: Stickstoffakkumulation in der unterirdischen Biomasse $(0-60 \mathrm{~cm})$ in den Versuchsjahren 2015 und 2016 am Standort Reinshof. Dargestellt sind die Mittelwerte \pm Standardabweichung. Varianten mit gleichen Großbuchstaben bzw. gleichen Kleinbuchstaben sind nicht signifikant verschieden, $p<0,05$ (Tukey-Test). Bei gestapelten Säulen beziehen sich die Angaben auf die Höhe der Gesamtsäule. n.v. = Werte nicht verfügbar.

Abbildung 15: Stickstoffakkumulation in der unterirdischen Biomasse $(0-30 \mathrm{~cm})$ in den Versuchsjahren 2015 und 2016 am Standort Schoningen. Dargestellt sind die Mittelwerte \pm Standardabweichung. Varianten mit gleichen Großbuchstaben bzw. gleichen Kleinbuchstaben sind nicht signifikant verschieden, $p<0,05$ (Tukey-Test). Bei gestapelten Säulen beziehen sich die Angaben auf die Höhe der Gesamtsäule. n.v. = Werte nicht verfügbar.

Abbildung 16: Nmin-Mengen in drei Tiefenstufen im Versuchsjahr 2015 am Standort Reinshof unterteilt in die Probenahme im Winter (a) und nach der Ernte im Sommer bzw. Herbst (b). Varianten mit gleichen Großbuchstaben (Winter-Nmin) bzw. gleichen Kleinbuchstaben (NachErnte-Nmin) sind nicht signifikant verschieden, $\mathrm{p}<0,05$ (Tukey-Test). Die statistischen Angaben beziehen sich auf die Höhe der Gesamtsäule.

Abbildung 17: Nmin-Mengen in drei Tiefenstufen im Versuchsjahr 2016 am Standort Reinshof unterteilt in die Probenahme im Winter (a) und nach der Ernte im Sommer bzw. Herbst (b). Varianten mit gleichen Großbuchstaben (Winter-Nmin) bzw. gleichen Kleinbuchstaben (NachErnte-Nmin) sind nicht signifikant verschieden, $\mathrm{p}<0,05$ (Tukey-Test). Die statistischen Angaben beziehen sich auf die Höhe der Gesamtsäule.

Abbildung 18: Nmin-Mengen in drei Tiefenstufen im Versuchsjahr 2015 am Standort Schoningen unterteilt in die Probenahme im Winter (a) und nach der Ernte im Sommer bzw. Herbst (b). Varianten mit gleichen Großbuchstaben (Winter-Nmin) bzw. gleichen Kleinbuchstaben (Nach-Ernte-Nmin) sind nicht signifikant verschieden, $p<0,05$ (Tukey-Test). Die statistischen Angaben beziehen sich auf die Höhe der Gesamtsäule. .51 
Abbildung 19: Nmin-Mengen in drei Tiefenstufen im Versuchsjahr 2016 am Standort Schoningen unterteilt in die Probenahme im Winter (a) und nach der Ernte im Sommer bzw. Herbst (b). Varianten mit gleichen Großbuchstaben (Winter-Nmin) bzw. gleichen Kleinbuchstaben (Nach-Ernte-Nmin) sind nicht signifikant verschieden, $\mathrm{p}<0,05$ (Tukey-Test). Die statistischen Angaben beziehen sich auf die Höhe der Gesamtsäule.

Abbildung 20: Menge an pflanzenverfügbarem Wasser nach der Ernte der Varianten in einer Bodentiefe von $80 \mathrm{~cm}$ unterteilt in drei Tiefenstufen in den Versuchsjahren 2015 und 2016 am Standort Reinshof, n.v. $=$ Werte nicht verfügbar.

Abbildung 21: Menge an pflanzenverfügbarem Wasser nach der Ernte der Varianten in einer Bodentiefe von $80 \mathrm{~cm}$ unterteilt in drei Tiefenstufen in den Versuchsjahren 2015 und $2016 \mathrm{am}$ Standort Schoningen, n.v. = Werte nicht verfügbar. .55

Abbildung 22: Gesellschaftliche Akzeptanz der untersuchten Feldfrüchte als Energiepflanzen für die Biogaserzeugung, $n=446$.

Abbildung 23: Teilindizes und Gesamtindex der relativen Anbauwürdigkeit (IrA). Mittelwerte aus den Versuchsjahren 2015 und 2016 am Standort Reinshof. Bei der mit * gekennzeichneten Variante geht nur das Versuchsjahr 2016 ein. Dargestellt sind die skalierten Werte für die Teilindizes sowie die daraus gebildete Summe (Gesamtindex IrA) .70

Abbildung 24: Teilindizes und Gesamtindex der relativen Anbauwürdigkeit (IrA). Mittelwerte aus den Versuchsjahren 2015 und 2016 am Standort Schoningen. Bei den mit * gekennzeichneten Varianten geht nur das Versuchsjahr 2016 ein. Dargestellt sind die skalierten Werte für die Teilindizes sowie die daraus gebildete Summe (Gesamtindex IrA)..70

Abbildung A1: Versuchsplan Standort Reinshof für die beiden Versuchsjahre 2015 und 2016. 144

Abbildung A2: Versuchsplan Standort Schoningen für die beiden Versuchsjahre 2015 und 2016. 


\section{Tabellenverzeichnis}

Tabelle 1: Korngrößenverteilung des Feinbodens an den Standorten Reinshof und Schoningen, Analyse mittels Siebung und Sedimentation nach DIN ISO 11277:2002-08, Bodenprobenahme zu Versuchsbeginn im Oktober 2014.

Tabelle 2: Bodenkennwerte und Grundnährstoffe im Oberboden $(0-30 \mathrm{~cm})$ an den beiden Versuchsstandorten Reinshof und Schoningen in den Versuchsjahren 2015 und 2016....... 6

Tabelle 3: Geprüfte Varianten in den Feldversuchen. ................................................

Tabelle 4: Termine der Aussaat und Ernte sowie Angaben zu Saatstärken und Reihenweiten der Varianten in den Versuchsjahren 2015 und 2016 an den Standorten Reinshof und Schoningen.

Tabelle 5: Geschlechtsverteilung, Altersverteilung, Bildungsabschluss sowie Angabe zur Naturverbundenheit der Umfrageteilnehmer $(n=446)$.

Tabelle 6: Ergebnisse der statistischen Analyse (ANOVA) der festen Effekte Variante und Jahr sowie deren Interaktion im linearen gemischten Modell für die erfassten Sprossdaten (Trockenmasseertrag, Trockensubstanzgehalt, N-Akkumulation, Methanflächenertrag, Cobalt- und Nickelgehalt) an den Standorten Reinshof und Schoningen.

Tabelle 7: Trockensubstanzgehalte in den Versuchsjahren 2015 und 2016 am Standort Reinshof.

Tabelle 8: Trockensubstanzgehalte in den Versuchsjahren 2015 und 2016 am Standort Schoningen.

Tabelle 9: Spezifischer Methanertrag der untersuchten Varianten am Standort Reinshof im zweiten Versuchsjahr bzw. bei der mehrjährigen Blühmischung zusätzlich im ersten Versuchsjahr. Analysemethode: „Hohenheimer Biogasertragstest“ nach VDI 4630.

Tabelle 10: Methanflächenerträge in den Versuchsjahren 2015 und 2016 an den Standorten Reinshof und Schoningen. .35

Tabelle 11: Cobalt- und Nickelgehalte im oberirdischen Aufwuchs in den Versuchsjahren 2015 und 2016 an den Standorten Reinshof (a) und Schoningen (b).

Tabelle 12: Ergebnisse der statistischen Analyse (ANOVA) der festen Effekte Variante und Jahr sowie deren Interaktion im linearen gemischten Modell für die erfassten Wurzeldaten (Wurzel-Trockenmasse, $\mathrm{C} / \mathrm{N}-$ Verhältnis der Wurzeln und N-Akkumulation in die unterirdische Biomasse) an den Standorten Reinshof und Schoningen. 38

Tabelle 13: C/N-Verhältnis der Wurzeln (0-60 cm Tiefe) in den Versuchsjahren 2015 und 2016 am Standort Reinshof.

Tabelle 14: C/N-Verhältnis der Wurzeln (0-30 cm Tiefe) in den Versuchsjahren 2015 und 2016 am Standort Schoningen.

Tabelle 15: Ergebnisse der statistischen Analyse (ANOVA) der festen Effekts Variante im linearen gemischten Modell für den erfassten Parameter Nmin an den Standorten Reinshof und Schoningen in den Versuchsjahren 2015 und 2016.

Tabelle 16: Bewertung der untersuchten Feldfüchte durch die Umfrageteilnehmer. Ergebnisse der Frequenzanalyse, Häufigkeitsangaben $(n=x)$ stellen das Auftreten der Kategorien im Fragebogenmaterial bei den einzelnen Feldfrüchten dar, Angaben in \%. ......58 
Tabelle 17: Kenngrößen der ökonomischen Bewertung (Leistung, Kosten, Deckungsbeitrag I) der untersuchten Varianten an den Standorten Reinshof (a) und Schoningen (b) in den Versuchsjahren 2015 und 2016.

Tabelle 18: Korrelationskoeffizienten ( $r$ ) ausgewählter Parameter für die vereinigten Werte aus den Versuchsjahren 2015 und 2016 sowie den Standorten Reinshof und Schoningen, $n=174$, Korrelation nach Pearson, Korrelationskoeffizienten signifikant bei $p \leq 0,05$. 62

Tabelle 19: Teilindizes des Index IrA für die erhobenen Parameter Trockenmasseertrag (TMErtrag), Trockensubstanzgehalt (TS-Gehalt), Methanflächenertrag, Nmin, WurzelTrockenmasse, Bodenwasser, Spurenelemente (Co, Ni) und ökonomische Aspekte (Deckungsbeitrag) in den Versuchsjahren 2015 und 2016 am Standort Reinshof

Tabelle 20: Teilindizes des Index IrA für die erhobenen Parameter Trockenmasseertrag (TMErtrag), Trockensubstanzgehalt (TS-Gehalt), Methanflächenertrag, Nmin, WurzelTrockenmasse, Bodenwasser, Spurenelemente (Co, Ni) und ökonomische Aspekte (Deckungsbeitrag) in den Versuchsjahren 2015 und 2016 am Standort Schoningen...........66

Tabelle 21: Teilindex Soziale Aspekte (Akzeptanz) der untersuchten Feldfrüchte. 69

Tabelle A1: Zusammensetzung des Saatgutes der mehrjährigen Blühmischung (BG70) mit ein- und mehrjährigen Arten, sortiert nach Gewichtsanteil der Arten in der Mischung........146

Tabelle A2: Zusammensetzung des Saatgutes der einjährigen Blühmischung (BG80), sortiert nach Gewichtsanteil der Arten in der Mischung.

Tabelle A3: Deckungsbeitragsrechnung am Beispiel von Mais am Standort Reinshof im Versuchsjahr 2016. 148

Tabelle A4: Zusammensetzung des Teilindex Nmin als Mittelwert aus Nmin (w), erhoben im Winter und Nmin (s/h), erhoben im Sommer bzw. Herbst nach der Ernte in den Versuchsjahren 2015 und 2016 a) am Standort Reinshof und b) am Standort Schoningen. Bei den Varianten des Zweikulturnutzungssystems (Wickroggen, Winterackerbohnen, Wintertriticale und Gemenge Winterackerbohnen/Wintertriticale) wurde der Nmin-Wert im Winter bei Winterungen und nach der Ernte nach der Zweitfrucht Mais ermittelt. Bei den Sommerhauptfrüchten (Sommerackerbohnen, Einjährige Blühmischung, Amarant, Mais und Gemenge Amarant/Mais) wurde der Nmin-Wert im Winter bei Schwarzbrache erhoben. Bei den mehrjährigen Arten (Deutsches Weidelgras, Mehrjährige Blühmischung, Durchwachsene Silphie) stellt das erste Versuchsjahr das Ansaat- bzw. Pflanzjahr dar. 149 
Im Jahr 2018 waren in Deutschland 9494 Biogasanlagen in Betrieb. Diese Anlagen haben damit einen wesentlichen Anteil an der Nutzung von Primärenergie, der über erneuerbare Energien bereitgestellt wurde, getragen (FNR 2019). Dabei wird überwiegend Silomais als Substrat eingesetzt. Im Jahr 2016 waren dies $69 \%$ des massebezogenen Substrateinsatzes. Geringere Anteile entfielen auf Grassilage und Ganzpflanzengetreide. Zu nur $1 \%$ wurden z.B. auch Zwischenfrüchte eingesetzt (FNR 2019).

Neben der Stromproduktion und Wärmenutzung sind im Biogasbereich aber auch neue, innovative Nutzungskonzepte in der Entwicklung. Der Landmaschinenhersteller New Holland z.B. sieht vor, Biomethan für den Antrieb landwirtschaftlicher Zugmaschinen zu nutzen (New Holland Agriculture 2020). Ende 2020 soll ein Methan-Schlepper, der laut Hersteller, hinsichtlich der Leistung mit einem konventionell betriebenen gleichauf ist, serienreif sein. So wäre, kombiniert möglicherweise mit der Nutzung der $\mathrm{N}_{2}$-Fixierung von Leguminosen, ein weitgehend geschlossener Energiekreislauf im Ackerbau möglich. Andere innovative Nutzungskonzepte für Biogasanlagen sehen die Kombination stofflicher und energetischer Nutzung von Biomasse vor. So schlagen Diekmann et al. (2018) vor, je nach Art der Feldfrucht zuerst nutzbare Stoffgruppen aus der Biomasse abzutrennen und den verbleibenden Rest energetisch zu verwerten. Abtrennbare Stoffgruppen sind dabei den Autoren zufolge Saccharide, Proteine, Lipide, Nährstoffe, Lignin und Sonderstoffe, z.B. Duft- oder Farbstoffe. Eine Weiterverarbeitung dieser Stoffgruppen ist in vielfältigen Bereichen, z.B. der Nahrungs- und Futtermittelindustrie oder zu kosmetischen und pharmazeutischen Zwecken, möglich.

Sauer (2010) konnte im Zusammenhang mit der Untersuchung einer mit Energiepflanzen betriebenen, instabil laufenden Praxisbiogasanlage, einen Mangel an Cobalt und Nickel identifizieren. Auch Pobeheim et al. (2011) konnten in einem Versuch zeigen, dass eine allein mit Mais als Substrat betriebene Versuchsbiogasanlage eine ungenügende Prozessstabilität aufweist und die Zugabe von Cobalt und Nickel diesem entgegenwirken kann. Neben diesen Spurenelementen sind weiterhin auch Eisen, Molybdän, Selen und Wolfram essenziell für methanogene Mikroorganismen und damit für die Prozessstabilität der Biogasanlage (Demirel \& Scherer 2011). Die Ursache für die Befunde liegt in der unzureichenden Fähigkeit von Mais und Getreide, gerade die zentralen Elemente Cobalt und Nickel aufzunehmen - ein Mangelzustand der methanbildenden Mikroorganismen als Folge (Sauer et al. 2014). Um in hauptsächlich mit den klassischen Energiepflanzen betriebenen Biogasanlagen diesem Mangel zu begegnen und die Biogasausbeute zu stabilisieren, werden in der Praxis Spurenelementpräparate eingesetzt. Der praktische Einsatz erscheint dabei nur wenig transparent, wie allein die weite Spanne der minimalen Grenzwerte vermuten lässt: Dermirel \& Scherer (2011) kommen bei einer Literaturzusammenschau zu einem Bereich von 0,05-0,19 bzw. 0,11-0,25 mg kg-1 Fermentermaterial für die beiden Schwermetalle Cobalt und Nickel. Auch Koch et al. (2011) stellten fest, dass die Dosierung dieser potenziell gefährlichen Substanzen gut begründet sein muss. Zudem wird für die Herstellung der Spurenelementpräparate das Cobalt unter umweltschädlichen und menschenunwürdigen Bedingungen, z.B. in der Demokratischen Republik Kongo, abgebaut (Mau 2019). 
Wie Sauer et al. (2014) zeigen konnten, gibt es Pflanzenarten, auch innerhalb der landwirtschaftlich genutzten, die anders als Mais und Getreide, die prozessrelevanten Spurenelemente Cobalt und Nickel in deutlich höheren Mengen aufnehmen können. In dem, dieser Arbeit zugrundeliegenden Verbundprojekt, „Spurenelemente durch Energiepflanzen - Stoffströme und Handlungsempfehlungen für eine optimierte Prozessbiologie in Biogasanlagen“ wurde daher die Hypothese aufgestellt, dass durch eine Mischung von Mais mit Pflanzen, die Spurenelemente akkumulieren, eine ausreichende Versorgung für die Vergärung gewährleistet ist und auf die Zugabe von Spurenelementpräparaten verzichtet werden kann. Zu diesen Arten zählen u.a. Ackerbohnen, sowohl die Winter- als auch die Sommerform, Amarant, Malve, Buchweizen und Zottelwicke (Sauer et al. 2014).

Ziel der pflanzenbaulichen Untersuchungen ist es, spurenelementreiche aber auch klassische Feldfrüchte auf ihre Anbauwürdigkeit als Energiepflanze für die Biogaserzeugung zu prüfen. Wichtige Kenngrößen für die Bewertung von Energiepflanzen für die Biogaserzeugung ergeben sich aus Problemen des verbreiteten intensiven Maisanbaus aber auch aus den Anforderungen an Energiepflanzen für die Biogaserzeugung:

- Versuchsergebnisse mit Schwerpunkt auf der pflanzenbaulichen, ökologischen und ökonomischen Leistung verschiedener Energiepflanzen-Anbausysteme zeigen, dass Mais an den meisten Standorten in ganz Deutschland das größte Potenzial hinsichtlich der Erzielung hoher Trockenmasseerträge und Methanausbeuten aufweist (Vetter 2009). Die Erträge sind wichtige Größen, da sie letztendlich den ökonomischen Erfolg bestimmen. Es ist fraglich, ob alternative Feldfrüchte die Leistungsfähigkeit von Mais erreichen oder diese sogar übersteigen können.

- Ein wichtiges Kriterium für die Eignung einer Feldfrucht als Energiepflanze für die Biogaserzeugung ist der Trockensubstanzgehalt (Förster et al. 2019). Für eine erfolgreiche Silierung sind Trockensubstanzgehalte im Bereich von 28-35 \% günstig. Bei Trockensubstanzgehalten darunter besteht die Gefahr von unerwünschten Sickersaftverlusten (Herrmann \& Rath 2012).

- Die Nitratverlagerung und -auswaschung stellt zwar unter Silomais selbst, zumindest bei bedarfsgerechter Düngung, kein Problem dar (Büchter et al. 2001). Werden aber Mais und andere Sommerhauptfrüchte nach Schwarzbrache über Winter angebaut, besteht in dieser Zeit die Gefahr der Nitratauswaschung, wenn hohe Mengen mineralischen Stickstoffs im Boden vorliegen (Meisinger \& Ricigliano 2017). Gleiches gilt für hohe Nmin-Mengen im Herbst im Boden, z.B. nach hohen Gaben von Wirtschaftsdüngern zu Mais. Mit der Bewertung der im Boden hinterlassenen Nmin-Mengen können möglicherweise auch Arten und Anbausysteme identifiziert werden, die hier eine günstige Wirkung entfalten.

- Silomais wird als humuszehrende Feldfrucht eingestuft (VDLUFA 2014). Die Humusbilanz ist bei Silomais trotz Gärrestrückführung durch den geringen Gärrestanfall aufgrund hoher Abbauraten negativ (KTBL 2012). Die Wurzeltrockenmasse der Feldfrüchte soll hier Aufschluss geben. 
- Die Spurenelementversorgung der Mikroorganismen im Biogasprozess kommt bei hauptsächlichem Input von Mais und Getreide in Mangel. Die Untersuchungen von Sauer et al. (2014) sind vielversprechend - möglicherweise können alternative Energiepflanzen hier einen Beitrag leisten.

- Für die landwirtschaftliche Praxis ist es bedeutsam, Kenntnis über den Wasserzustand des Bodens zu haben, den eine Feldfrucht nach der Ernte zurücklässt. Dies hat nicht zuletzt Auswirkungen auf die Keimung und Entwicklung der Folgefrucht. So besteht z.B. die Gefahr im Zweikulturnutzungssystem, dass bei ungünstiger Niederschlagsverteilung der Zweitfrucht nicht genügend Wasser zur Verfügung steht.

- Da der insbesondere mit dem Ausbau von Biogasanlagen zusammenfallende Anstieg der Maisanbauflächen von der Bevölkerung als negative Auswirkung auf das Landschaftsbild empfunden wird (Wiehe et al. 2009), kann hier angesetzt werden, um die Haltung gegenüber anderen Feldfrüchten einschätzen zu können.

Menke (2011) entwickelte den Index der relativen Anbauwürdigkeit - IrA - an Winterzwischenfrüchten im Zweikulturnutzungssystem mit Mais. Drei Teilindizes gingen bei diesem Autor in den Index IrA ein: Nmin-Index, Winterzwischenfrucht-Index und Energiemais-Index. Dabei wurden im Teilindex Nmin die Nmin-Mengen im Winter unter Winterzwischenfrüchten berücksichtigt, beim Teilindex Winterzwischenfrucht der Methanertrag der Winterzwischenfrüchte bei der Biomasseernte im Mai und beim Teilindex Energiemais der Trockenmasseertrag von Mais als Zweitfrucht nach den Winterzwischenfrüchten. Unter Einbeziehung der vorgestellten Kenngrößen, die beim Anbau von Energiepflanzen von Bedeutung sind, aber auch mit Blick auf verschiedene Anbausysteme, soll in der vorliegenden Untersuchung der Index IrA weiterentwickelt werden. Mit dem sich daraus ergebenden umfassenden Bewertungsschema soll eine gut begründete Einschätzung der Wirkung der Feldfrüchte als Energiepflanzen für die Biogaserzeugung erfolgen.

Ein Verlust an Biodiversität kann mit einer Verringerung von Ökosystemfunktionen, Stabilität, Widerstandsfähigkeit und Produktivität in Verbindung gebracht werden (Cardinale et al. 2012). Die Herausforderungen im Zuge des Klimawandels, die Variabilität des Klimas und die Zunahme von extremen Wetterereignissen wie Trockenheit bis hin zur Dürre, Hitze und Starkregen können gravierende Folgen für die landwirtschaftliche Produktion haben und erfordern Anpassungsstrategien (IPCC 2019).

Vielfältige Produktionssysteme sowie der Anbau eines vielfältigen Artenspektrums können zur Anpassung an veränderte Bedingungen und zur Erhöhung der Resilienz beitragen: Bei der Untersuchung von Zweikulturnutzungssystemen im Rahmen des Energiepflanzenanbaus fanden Graß et al. (2013) eine höhere Ertragsstabilität beim Anbau von zwei Feldfrüchten innerhalb eines Jahres (Erst- und Zweitfrucht) im Vergleich zu den Hauptkulturen. Im Zweikulturnutzungssystem kann sich, so die Autoren, auch das Risiko von Wetterextremen auf zwei Feldfrüchte (oder entsprechend mehr, wenn Gemenge angebaut werden) verteilen, was zu einer höheren Ertragsstabilität führt. Gensch (2019) stellte fest, dass eine Zwischenfruchtmischung im Vergleich zu einer Reinsaat günstigere Effekte auf den Bodenwasserhaushalt hat. Der Autor begründet dies mit einem durch die unterschiedlichen Stockwerke entstehenden Mikrokli- 
mas, das die Feuchtigkeit nahe der Bodenoberfläche hält. So konnte die Zwischenfruchtmischung effizient das Wasser ausnutzen. Auch das Wasserdefizit durch den Zwischenfruchtanbau konnte bis zur Maisaussaat ausgeglichen und der Bodenwassergehalt im Vergleich zur Schwarzbrache sogar erhöht werden. Anhand einer Meta-Analyse konnten Raseduzzaman \& Jensen (2017) feststellen, dass der Gemengeanbau aus Leguminosen und Nicht-Leguminosen in allen Klimazonen eine höhere Ertragsstabilität zeigt als der Anbau der beiden Reinsaaten. Somit kann auch der Gemengeanbau von Getreide und Körnerleguminosen und die damit zusammenhängende Diversifizierung des Nutzpflanzenspektrums die Resilienz von landwirtschaftlichen Produktionssystemen erhöhen.

Da jede Pflanze Energie speichert, ist auch jede Pflanze eine potenzielle Energiepflanze (Karpenstein-Machan 2005). Vor diesem Hintergrund wäre es gerade im Energiepflanzenanbau möglich, eine hohe Vielfalt an Pflanzenarten in Ackerbausystemen zu realisieren. Für den Anbau von Energiepflanzen zur Biogaserzeugung wurden im Jahr 2019 etwa 1,55 Mio. Hektar Fläche in Deutschland genutzt. Wie die Anbauflächen der einzelnen Feldfrüchte zur Biogaserzeugung zeigen, wird dabei der größte Teil mit nur wenig Vielfalt genutzt. Die Anbaufläche setzt sich zusammen aus einem überwiegenden Anteil Mais und zu kleineren Anteilen aus Getreide und Gräsern (FNR 2020). Andere Energiepflanzen für die Biogaserzeugung haben derzeit nur wenig Bedeutung. Die Durchwachsene Silphie z.B. hatte 2019 einen Flächenanteil von gerade einmal 0,2\% (FNR 2020).

Im Rahmen der vorliegenden Untersuchung sollte folgenden Forschungsfragen nachgegangen werden:

Welche der untersuchten Feldfrüchte eignen sich als Energiepflanzen für die Biogaserzeugung? Können Feldfrüchte empfohlen werden, die das aktuelle Energiepflanzenspektrum erweitern?

Eignet sich der Index IrA als Werkzeug zur Bewertung von Energiepflanzen für die Biogaserzeugung? Welche Kenngrößen sind wichtig für die Bewertung von Pflanzen als Energiepflanzen für die Biogaserzeugung und sollten als Teilindizes im Index IrA abgebildet werden? 


\section{$2.1 \quad$ Feldversuche}

\subsubsection{Standorteigenschaften und Witterung}

Es wurden zweijährige Feldversuche, 2014/2015 und 2015/2016, im Weiteren bezeichnet mit 2015 für das erste und 2016 für das zweite Versuchsjahr an zwei Standorten in Südniedersachsen angelegt: Reinshof und Schoningen. Der Reinshof ist ein Versuchsgut der Universität Göttingen und liegt etwa 4 km südlich vom Zentrum Göttingens entfernt im Leinetal. Die Feldversuche wurden an diesem Standort auf dem Garteschlag (N 51²9'23.41", E 956 '11.95", 164 m über NN) durchgeführt. Der Bodentyp ist eine Schwarzerde-Parabraunerde, entstanden aus Lösslehmen. Mit einer Ackerzahl von 89 handelt es sich hier um einen sehr fruchtbaren Standort. Schoningen befindet sich etwa $3 \mathrm{~km}$ südlich von Uslar an den Südausläufern des Sollings, einem Mittelgebirge im Weserbergland. Die Feldversuche wurden hier auf dem Schlag Sömmerling (N 51³8`26.37", E 940`15.46", 251 m über NN), einem Schlag eines ortsansässigen Praxisbetriebs, durchgeführt. Beim Bodentyp handelt es sich eine PseudogleyBraunerde, entstanden aus Buntsandsteinverwitterung. Ackerzahlen zwischen 43 und 51 weisen auf eine mittlere Fruchtbarkeit des Standorts hin.

Die Bodenart wird für den Standort Reinshof in den Tiefen 0-30 und 30-60 cm als stark toniger Schluff klassifiziert (Tabelle 1). Am Standort Schoningen enthält der Boden höhere Sandanteile als am Reinshof. Der Oberboden $(0-30 \mathrm{~cm})$ am Standort Schoningen ist lehmiger, schluffiger Sand, der Unterboden $(30-60 \mathrm{~cm})$ schwach sandiger Lehm.

Tabelle 1: Korngrößenverteilung des Feinbodens an den Standorten Reinshof und Schoningen, Analyse mittels Siebung und Sedimentation nach DIN ISO 11277:2002-08, Bodenprobenahme zu Versuchsbeginn im Oktober 2014.

\begin{tabular}{ccccc}
\hline $\begin{array}{c}\text { Bodentiefe } \\
(\mathrm{cm})\end{array}$ & Sand (\%) & Schluff (\%) & Ton (\%) & Bodenart \\
\hline \multicolumn{5}{c}{ Reinshof } \\
$0-30$ & 12,7 & 69,4 & 17,9 & Ut4 \\
$30-60$ & 11,2 & 67,5 & 21,3 & Ut4 \\
\hline \multicolumn{6}{c}{ Schoningen } \\
$0-30$ & 33,5 & 49,8 & 16,7 & Slu \\
$30-60$ & 35,6 & 41,7 & 22,7 & Ls2 \\
\hline
\end{tabular}

Tabelle 2 zeigt die Bodenkennwerte (Corg, Gesamt-N, Humus), erfasst zu Versuchsbeginn im Oktober 2014, sowie die Grundnährstoffe und pH-Werte im Oberboden für die beiden Versuchsjahre 2015 und 2016 der Versuchsflächen Reinshof und Schoningen. Hinsichtlich der Gehalte an Corg, Gesamt-N und Humus unterschieden sich die beiden Standorte kaum und sind mit $<2 \%$ Humus schwach humos. Die Grundnährstoffanalyse des Oberbodens $(0-30 \mathrm{~cm})$ ergab überwiegend mittlere bis hohe Versorgungsstufen bei den Nährstoffen Phosphat und Magnesium an beiden Standorten und Kaliumoxid zumindest am Standort Schoningen (Gehaltsklasse C-D). Geringere Mengen als angestrebt zeigten sich am Standort Reinshof für Kaliumoxid (Gehaltsklasse B). Der pH-Wert bewegte sich in beiden Versuchsjahren an beiden 
Standorten im schwach sauren Bereich. Die größte Abweichung vom angestrebten $\mathrm{pH}-\mathrm{Wert} 7$ zeigte sich mit einem pH-Wert von 6,08 im zweiten Versuchsjahr am Standort Schoningen.

Tabelle 2: Bodenkennwerte und Grundnährstoffe im Oberboden $(0-30 \mathrm{~cm})$ an den beiden Versuchsstandorten Reinshof und Schoningen in den Versuchsjahren 2015 und 2016.

\begin{tabular}{|c|c|c|c|c|c|c|c|}
\hline $\begin{array}{l}\text { Versuchs- } \\
\text { jahr }\end{array}$ & Corg (\%) & $\begin{array}{c}\text { Gesamt } \\
\mathrm{N}(\%)\end{array}$ & $\begin{array}{c}\text { Humus } \\
(\%)\end{array}$ & $\mathrm{pH}$ & $\mathrm{P}_{2} \mathrm{O}_{5}{ }^{*}$ & $\mathrm{~K}_{2} \mathrm{O}^{*}$ & $\mathrm{Mg}^{*}$ \\
\hline \multicolumn{8}{|c|}{ Reinshof } \\
\hline 2015 & 1,10 & 0,14 & 1,89 & 6,76 & $15,34(\mathrm{C})$ & $9,50(\mathrm{~B})$ & $11,81(\mathrm{D})$ \\
\hline 2016 & & & & 6,48 & $16,04(\mathrm{C})$ & $12,05(B)$ & $12,00(\mathrm{D})$ \\
\hline \multicolumn{8}{|c|}{ Schoningen } \\
\hline 2015 & 1,10 & 0,13 & 1,90 & 6,42 & $11,79(\mathrm{C})$ & 20,82 (D) & 7,09 (D) \\
\hline 2016 & & & & 6,08 & $12,03(\mathrm{C})$ & $16,23(\mathrm{C})$ & $6,00(\mathrm{C})$ \\
\hline
\end{tabular}

* in mg/100 g getrockneter Boden, CAL-Methode, in Klammern dargestellte Gehaltsklassen nach VDLUFA

Zur Bestimmung der standortspezifischen Menge an pflanzenverfügbarem Wasser wurden an beiden Versuchsstandorten Gruben bis $80 \mathrm{~cm}$ Bodentiefe ausgehoben. Aufgeteilt in Abschnitte mit je $10 \mathrm{~cm}$ Stärke wurden mithilfe von Stechzylindern (100 $\mathrm{cm}^{3}$ Volumen) Bodenproben entnommen. Unter Anwendung der Druckmethode nach L. A. Richards 1941 (Amelung et al. 2018) wurden pF-Kurven (Wasserspannungskurven) für jede Stechzylinderprobe angefertigt. Zusammengefasst für die Bodenabschnitte 0-30, 30-60 und 60-80 cm und einer Wasserspannung entsprechend der nutzbaren Feldkapazität von pF 1,8 (Feldkapazität) bis pF 4,2 (Permanenter Welkepunkt) ergaben sich die in Abbildung 1 dargestellten pF-Kurven.

Am Standort Reinshof war das Bodenwasser in den beiden oberen Bodenabschnitten (0-30 und $30-60 \mathrm{~cm}$ ) bei einem Bodenwassergehalt von 15 bzw. 17 Vol.-\% bis etwa 35 Vol.-\% nutzbar (Abbildung 1A). Der Verlauf der pF-Kurve des unteren Abschnitts $(60-80 \mathrm{~cm})$ war den oberen Bodenabschnitten ähnlich, jedoch etwas nach links, zu geringeren Wassergehalten, verschoben. Die Verfügbarkeit des Bodenwassers am Standort Schoningen lag bei einem Bodenwassergehalt von 12 bzw. 14 Vol.-\% bis 35 Vol.-\% in den Bodenabschnitten 0-30 und 30$60 \mathrm{~cm}$ (Abbildung 1B). Im unteren Bodenabschnitt $(60-80 \mathrm{~cm}$ ) war das Bodenwasser hingegen nur bei sehr hohen Wassergehalten pflanzenverfügbar (25-34 Vol.-\%). Aus der nutzbaren Feldkapazität $\left(\mathrm{mm} \mathrm{dm}^{-1}\right)$ und der Bodentiefe $(\mathrm{dm})$ errechnet sich die theoretisch mögliche Menge an pflanzenverfügbarem Wasser. Diese beträgt in einer Tiefe von 0-80 cm am Standort Reinshof 159,56 mm, am Standort Schoningen 148,25 mm. Der effektive Wurzelraum liegt am Standort Reinshof bei 0-110 cm, am Standort Schoningen bei etwa 0-80 cm Tiefe (Landesamt für Bergbau, Energie und Geologie 2019). Aufgrund des im Vergleich zu der im vorliegenden Versuch untersuchten Bodentiefe $(0-80 \mathrm{~cm})$ deutlich tieferen effektiven Wurzelraums am Standort Reinshof ist hier auch eine höhere Menge pflanzenverfügbaren Wassers vorhanden als angegeben. Das Landesamt für Bergbau, Energie und Geologie (2019) gibt für den effektiven Wurzelraum an diesem Standort eine Menge an pflanzenverfügbarem Wasser von 250$300 \mathrm{~mm}$ an, für den Standort Schoningen $150 \mathrm{~mm}$, was das eigene Ergebnis bestätigt. Bei den vorliegenden Untersuchungen haben sich am Standort Schoningen bereits unterhalb des Pflughorizonts $(>30 \mathrm{~cm}$ ) hohe Steingehalte gezeigt, sodass hier von einer eingeschränkten Durchwurzelbarkeit unterhalb von $30 \mathrm{~cm}$ Tiefe ausgegangen werden kann, was wiederum Auswirkungen auf die Menge pflanzenverfügbaren Wassers hätte. Systematische Untersuchungen des Steingehalts erfolgten jedoch nicht. 


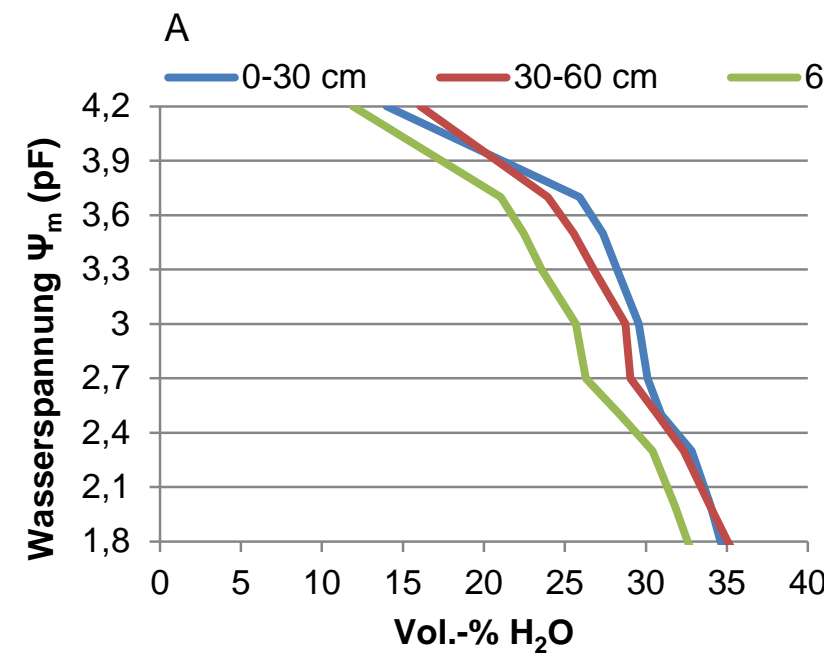
$60-80 \mathrm{~cm}$

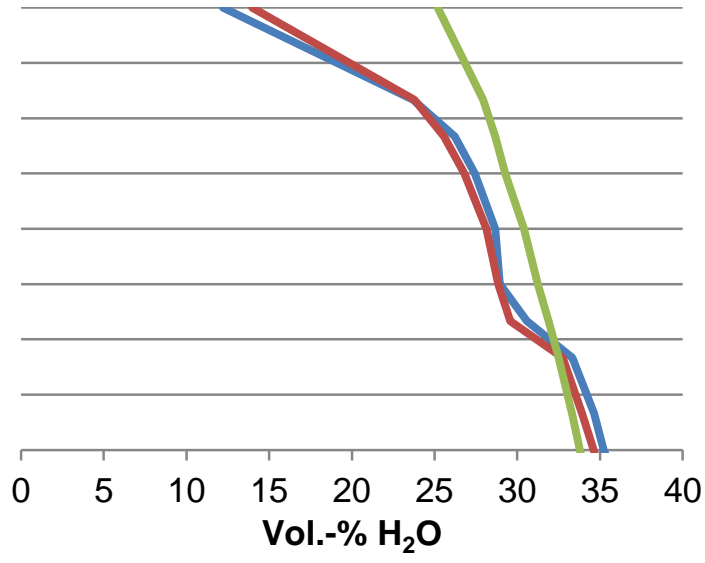

Abbildung 1: Beziehung zwischen Wassergehalt und Wasserspannung für den Boden am Standort Reinshof (A) und den Boden am Standort Schoningen (B). Unterteilung in die Bodenabschnitte 0-30 cm, 30-60 cm und 60-80 $\mathrm{cm}$.

Die Witterungsdaten wurden über den Deutschen Wetterdienst herangezogen. Am Standort Reinshof befindet sich die Wetterstation „Gartemühle“ in Nachbarschaft zum Versuchsfeld. Am Standort Schoningen wurden die Daten der Wetterstation Uslar für den Niederschlag und die Daten der etwa $5 \mathrm{~km}$ entfernten Wetterstation Wahlsburg-Lippoldsberg (176 m über NN) für die Temperatur genutzt.

Die gemessenen Jahresniederschlagssummen betrugen in den Referenzperioden 1961-1990 sowie 1981-2010 im Mittel über die Jahre am Standort Göttingen 645 bzw. 651 mm. Die Jahresdurchschnittstemperatur betrug hier in den angegebenen Zeiträumen 8,7 bzw. 9,2 ${ }^{\circ} \mathrm{C}$. An der Wetterstation Uslar wurden in der Referenzperiode 1961-1990 Jahresniederschlagssummen von durchschnittlich 841 mm ermittelt. Im Zeitraum von 1991-2010 waren es im Mittel 892 $\mathrm{mm}$. Die Jahresdurchschnittstemperatur an der Wetterstation Wahlsburg-Lippoldsberg betrug in der Referenzperiode 1991-2010 9,0 ${ }^{\circ} \mathrm{C}$. Daten der Jahre 1961-1990 sind für diesen Standort nicht verfügbar (DWD Climate Data Center 2019). Damit ist es am Standort Reinshof im Jahresdurchschnitt etwas wärmer als am Standort Schoningen. Hinsichtlich der jährlichen Niederschlagssummen unterscheiden sich die beiden Standorte jedoch deutlich mit bis zu $241 \mathrm{~mm}$ (Referenzperiode 1991-2010). Eine unterschiedliche Niederschlagsverteilung zwischen den Standorten zeigte sich auch während des Versuchszeitraums, wobei in Schoningen in der Mehrzahl der Monate mehr Niederschlag zu verzeichnen war als am Reinshof (Abbildung 2). Die Niederschläge wichen an beiden Standorten nahezu über die gesamte Versuchszeit von den Werten des langjährigen Mittels ab. In einigen Fällen waren die Niederschläge deutlich geringer als im langjährigen Mittel (z.B. im November 2014, Mai und Juni 2015 sowie Juli bis September 2016) und in einigen Fällen deutlich höher (z.B. im Juli und August 2015 sowie Juni 2016). 


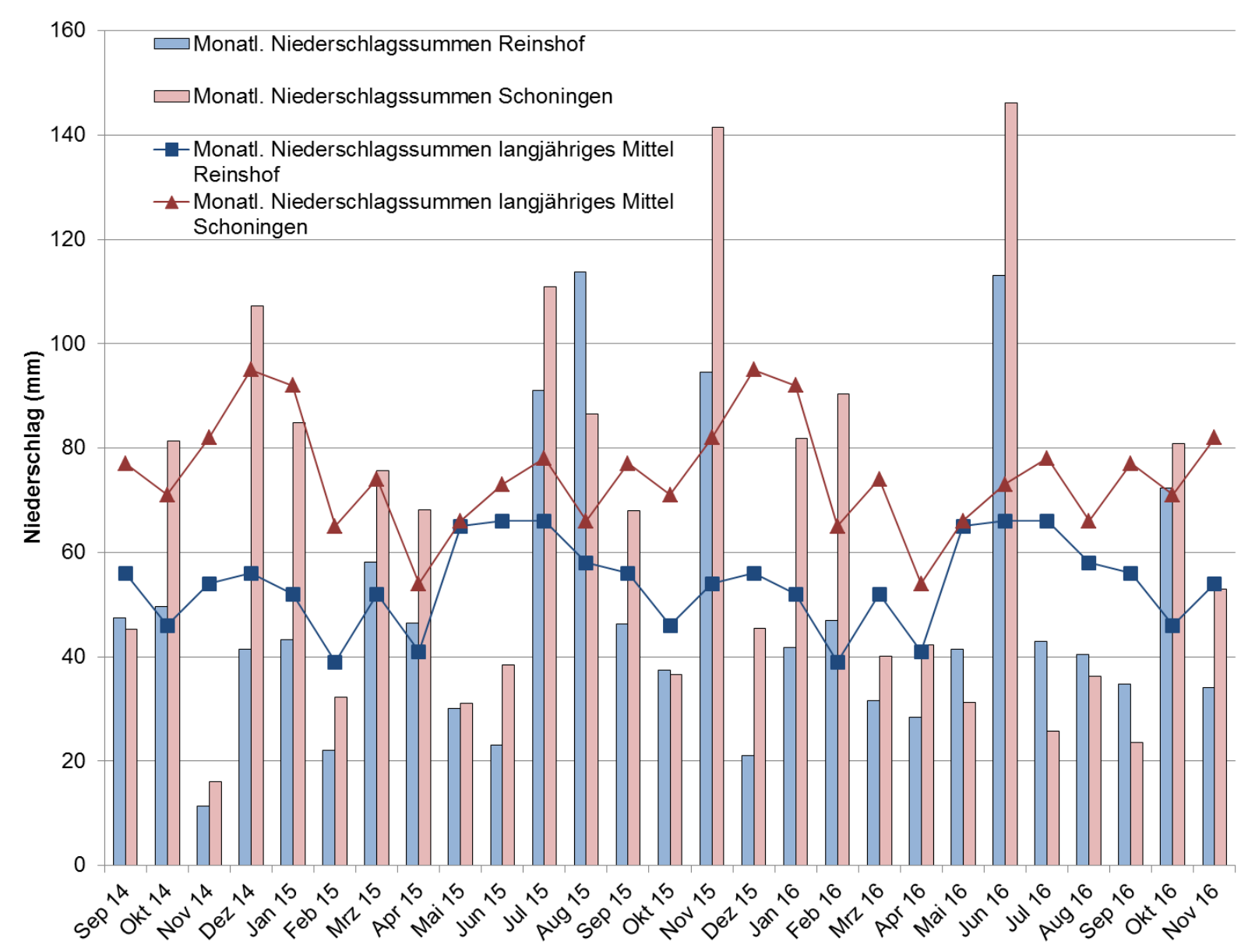

Abbildung 2: Monatliche Niederschlagssummen im Versuchszeitraum und im langjährigen Mittel (1981-2010). Standort Reinshof: Wetterstation Gartemühle, Standort Schoningen: Wetterstation Uslar (Deutscher Wetterdienst).

Auch die Lufttemperaturen im Versuchszeitraum wichen teilweise deutlich von den Werten des langjährigen Mittels ab (Abbildung 3). Im ersten Versuchsjahr waren die Lufttemperaturen im Herbst und z.T. im Winter an beiden Versuchsstandorten vergleichsweise mild. Auch in den Sommermonaten Juli und August waren höhere Temperaturen zu verzeichnen als im langjährigen Mittel. Das zweite Versuchsjahr begann ebenfalls mit einem milden Herbst an beiden Versuchsstandorten. Im Zeitraum von Mai bis September 2016 lagen die Temperaturen überwiegend über dem langjährigen Mittel. 


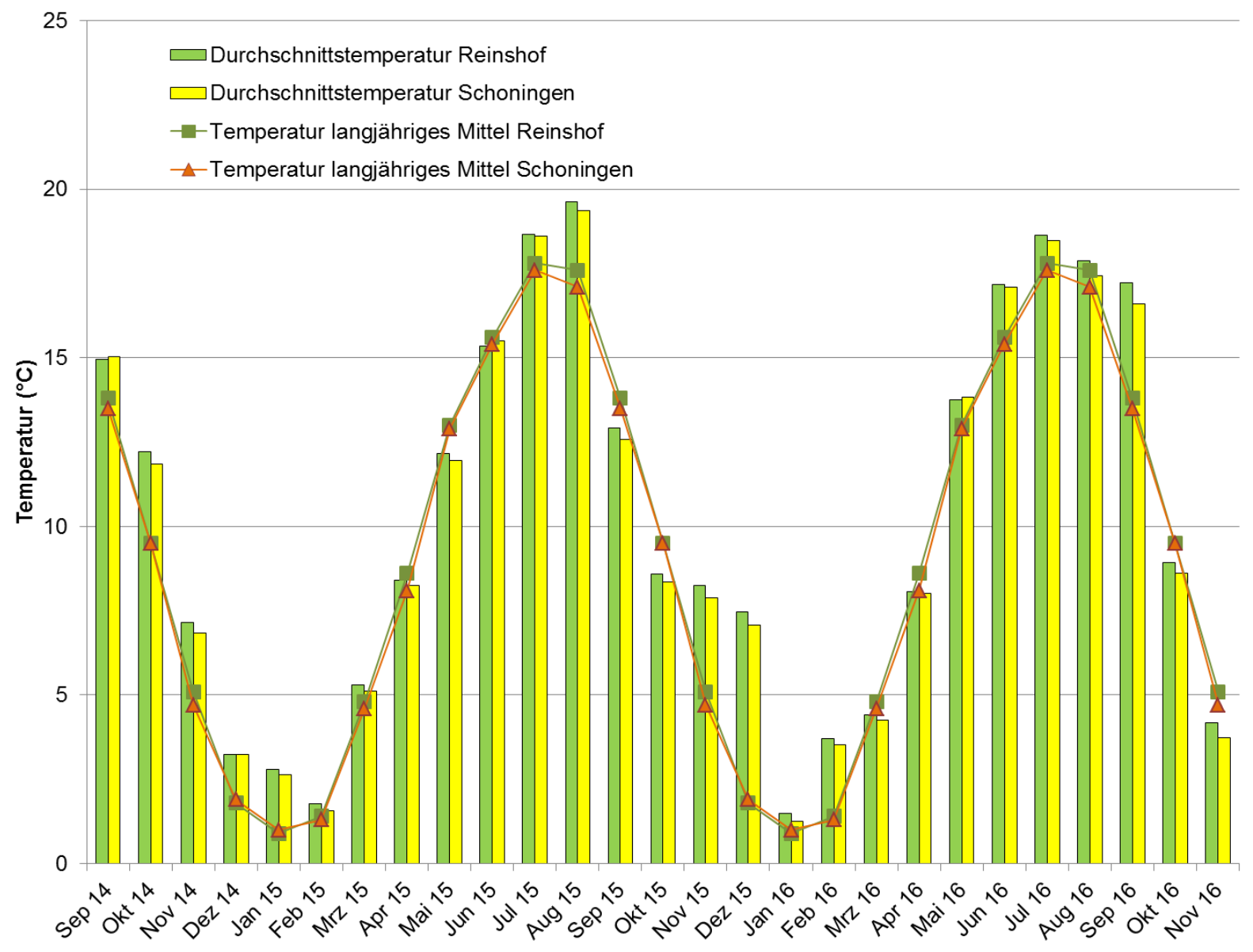

Abbildung 3: Monatliche Mittelwerte der Lufttemperatur im Versuchszeitraum und im langjährigen Mittel (19812010). Standort Reinshof: Wetterstation Gartemühle, Standort Schoningen: Wetterstation Wahlsburg-Lippoldsberg (Deutscher Wetterdienst).

\subsubsection{Versuchsaufbau}

Das Design des Versuchs war ein Semi-Lateinisches Quadrat mit vier Wiederholungen (Abbildung A1 und A2 im Anhang). Die Blockbildung erfolgt hierbei sowohl in horizontaler als auch in vertikaler Richtung. Eine Erfassung von Störgrößen in zwei Richtungen war damit möglich. Der Versuch war so angelegt, dass die Parzellen beider Versuchsjahre auf der gleichen Fläche randomisiert vorlagen. Die mehrjährigen Varianten konnten dadurch als Dauerkulturen über die gesamte Versuchszeit angebaut und untersucht werden. Die Vorfrucht im ersten Versuchsjahr war Winterweizen am Standort Reinshof bzw. Winterraps am Standort Schoningen. Auf den Parzellen der einjährigen Arten des zweiten Versuchsjahres wurde im ersten Versuchsjahr Winterweizen angebaut, sodass die Vorfrucht im zweiten Versuchsjahr an beiden Standorten einheitlich Winterweizen war.

Jede Parzelle $\left(67,5 \mathrm{~m}^{2}\right)$ bestand am Standort Reinshof aus sechs nebeneinanderliegenden Beeten mit einer Breite von jeweils 1,5 m und einer Länge von 7,5 m. Am Standort Schoningen war jede Parzelle $\left(33,75 \mathrm{~m}^{2}\right)$ in der Breite halbiert (drei Beete). Am Reinshof war die Ernte größerer Mengen Biomasse bzw. die Bereitstellung von Silagen vorgesehen, weshalb ein gröBerer Flächenumfang nötig war. 


\subsubsection{Versuchsglieder}

Angebaut wurden die Feldfrüchte in verschiedenen Anbausystemen (Tabelle 3). Es gab vier Varianten, bei denen Winterzwischenfrüchte bzw. Gemenge aus diesen im Zweikulturnutzungssystem mit Mais als Zweitfrucht angebaut wurden. Weitere fünf Varianten wurden als Sommerhauptfrüchte angebaut sowie drei mehrjährige Varianten als Dauerkulturen.

Tabelle 3: Geprüfte Varianten in den Feldversuchen.

\begin{tabular}{|c|c|c|c|}
\hline Nr. & Variante & $\begin{array}{l}\text { Botanische } \\
\text { Bezeichnung }\end{array}$ & Sorte \\
\hline & $\begin{array}{l}\text { Zweikulturnutzungssystem: Winter- } \\
\text { zwischenfrüchte mit } \\
\text { Mais als Zweitfrucht* }\end{array}$ & & \\
\hline 1 & $\begin{array}{l}\text { Wickroggen (Gemenge Winterroggen / } \\
\text { Winterwicke) }\end{array}$ & $\begin{array}{l}\text { Secale cereale } \\
\text { Vicia villosa }\end{array}$ & $\begin{array}{l}\text { Conduct } \\
\text { Welta }\end{array}$ \\
\hline 2 & Winterackerbohnen & Vicia faba & Nordica \\
\hline 3 & Wintertriticale & Triticosecale & Balu \\
\hline \multirow[t]{2}{*}{4} & $\begin{array}{l}\text { Gemenge Winterackerbohnen / } \\
\text { Wintertriticale }\end{array}$ & $\begin{array}{l}\text { Vicia faba } \\
\text { Triticosecale }\end{array}$ & $\begin{array}{l}\text { Nordica } \\
\text { Balu }\end{array}$ \\
\hline & Sommerhauptfrüchte & & \\
\hline 5 & Sommerackerbohnen & Vicia faba & Fanfare \\
\hline 6 & Einjährige Blühmischung & 12 Arten (einjährig) ${ }^{\star *}$ & BG80 \\
\hline 7 & Amarant & Amaranthus cruentus & Bärnkrafft \\
\hline 8 & Mais & Zea mays & Amadeo \\
\hline \multirow[t]{2}{*}{9} & $\begin{array}{l}\text { Gemenge Amarant / } \\
\text { Mais }\end{array}$ & $\begin{array}{l}\text { Amaranthus cruentus } \\
\text { Zea mays }\end{array}$ & $\begin{array}{l}\text { Bärnkrafft } \\
\text { Amadeo }\end{array}$ \\
\hline & Mehrjährige Arten & & \\
\hline 10 & Deutsches Weidelgras & Lolium perenne & Alligator \\
\hline 11 & Mehrjährige Blühmischung & $\begin{array}{l}25 \text { Arten (ein- und mehr- } \\
\text { jährig) }\end{array}$ & BG70 \\
\hline 12 & Durchwachsene Silphie & Silphium perfoliatum & Chrestensen \\
\hline
\end{tabular}

* Als Zweitfrucht wurden im ersten Versuchsjahr die Maissorte Simpatico und im zweiten Versuchsjahr die Maissorten Cathy (Reinshof) und P7326 (Schoningen) angebaut.

** Die Artenzusammensetzung der Blühmischungen wird im Anhang (Tabelle A1 und A2) dargestellt.

Zusätzlich zu diesen Varianten wurde im Versuchsjahr 2016 ein Zusatzversuch zum Test einer weiteren Amarantsorte bzw. Amarantart angelegt. Bei dem aus Russland stammenden Amarant Pastewny handelt es sich um die Art Amaranthus hybridus mit besonderer Eignung zur Futternutzung (Kaul et al. 1996). Die Amarantsorte Pastewny wurde, wie auch die Sorte Bärnkrafft, sowohl in Reinsaat als auch im Gemenge mit Mais getestet. Es gab vier Wiederholungen. In die statistische Analyse des Hauptversuchs wurde der Zusatzversuch nicht eingebunden.

\subsubsection{Versuchsdurchführung}

Die Flächen der Feldversuche wurden konventionell bewirtschaftet. Nach einer flachen Einarbeitung der Vorfrucht wurden die Flächen an beiden Standorten vor Versuchsbeginn im Herbst 
2014 gepflügt. Im zweiten Versuchsjahr erfolgte die Grundbodenbearbeitung nach der Stoppelbearbeitung mit einem Grubber. Zur Aussaat der Sommerhauptfrüchte sowie zur Aussaat bzw. Pflanzung der mehrjährigen Arten wurde der Boden nach Schwarzbrache über Winter ein weiteres Mal gegrubbert. Zur Saatbettbereitung wurde eine Kreiselegge eingesetzt. Die Aussaat wurde mit einer Parzellendrillmaschine durchgeführt und fand je nach Variante im Herbst 2014 bzw. 2015 sowie im Frühjahr 2015 bzw. 2016 statt (Tabelle 4). Die Varianten Wickroggen und Deutsches Weidelgras fielen aufgrund von starken Schäden an den Keimpflanzen durch Schneckenfraß im Oktober 2014 am Standort Schoningen im ersten Versuchsjahr aus. Das Deutsche Weidelgras wurde hier im Frühjahr 2015 erneut ausgesät. Wegen der ungleichen Aussaattermine des Deutschen Weidelgrases an den Standorten Reinshof und Schoningen fand die erste Erfassung der zu erhebenden Parameter in Schoningen erst im zweiten Versuchsjahr statt.

Die Varianten Wickroggen, Wintertriticale, ein- und mehrjährige Blühmischung, Amarant und Deutsches Weidelgras wurden achtreihig (acht Reihen je Beet), mit einem Reihenabstand von $17 \mathrm{~cm}$ ausgesät. Die Varianten Winterackerbohnen, Gemenge Winterackerbohnen / Wintertriticale und Sommerackerbohnen wurden sechsreihig (sechs Reihen je Beet), mit einem Reihenabstand von $22 \mathrm{~cm}$ ausgesät und die Varianten Mais und Durchwachsene Silphie zweireihig (zwei Reihen je Beet) mit einem Reihenabstand von $75 \mathrm{~cm}$. Bei dem Gemenge aus Amarant und Mais wurde der Mais zweireihig mit $75 \mathrm{~cm}$ Reihenabstand ausgesät und jeweils rechts und links davon eine Reihe Amarant. Die Durchwachsene Silphie wurde als Jungpflanze per Hand gepflanzt. Vor Mais als Zweitfrucht nach Winterzwischenfrüchten wurde der Boden flach mit einer Fräse bearbeitet. Als Zweitfrucht wurde im ersten Versuchsjahr die Maissorte Simpatico (S250) verwendet. Diese Maissorte reifte im ersten Versuchsjahr nicht ausreichend ab und wurde im zweiten Versuchsjahr durch die Sorten Cathy (Reinshof, S210) und Pioneer P7326 (Schoningen, S180) für die Zweitfruchtstellung ersetzt.

Die Nährstoffversorgung wurde durch eine Kombination aus organischer und mineralischer Düngung sichergestellt. Im März 2015 wurden am Standort Reinshof $60 \mathrm{~kg} \mathrm{~N}^{-1}, 25 \mathrm{~kg} \mathrm{P}_{2} \mathrm{O}_{5}$ ha $^{-1}$ und $85 \mathrm{~kg} \mathrm{~K}_{2} \mathrm{O}$ ha-1 über Gärreste auf allen Parzellen ausgebracht. Am Standort Schoningen waren es $31 \mathrm{~kg} \mathrm{~N}$ ha-1 $^{-1} 24 \mathrm{~kg} \mathrm{P}_{2} \mathrm{O}_{5}$ ha $^{-1}$ und $65 \mathrm{~kg} \mathrm{~K}_{2} \mathrm{O}$ ha $^{-1}$. Auch im zweiten Versuchsjahr erfolgte eine Gärrestausbringung (März 2016), bei der am Standort Reinshof $70 \mathrm{~kg} \mathrm{~N} \mathrm{ha}^{-1}, 44$ $\mathrm{kg} \mathrm{P}_{2} \mathrm{O}_{5}$ ha $^{-1}$ und $90 \mathrm{~kg} \mathrm{~K}_{2} \mathrm{O}$ ha $^{-1}$ und am Standort Schoningen $37 \mathrm{~kg} \mathrm{~N} \mathrm{ha}^{-1}, 24 \mathrm{~kg} \mathrm{P}_{2} \mathrm{O}_{5} \mathrm{ha}^{-1}$ und $74 \mathrm{~kg} \mathrm{~K}_{2} \mathrm{O}$ ha $^{-1}$ ausgebracht wurden. Der Stickstoffbedarf der jeweiligen Variante wurde, wenn nötig, zusätzlich über mineralische Düngung gedeckt. Zur Orientierung der Düngehöhe wurde der Stickstoff-Sollwert der einzelnen Arten bzw. Empfehlungen aus der Literatur verwandt. Die zu Vegetationsbeginn vorhandenen Mengen an mineralischem Stickstoff im Boden (Nmin) wurden ebenfalls bei der Ermittlung der Düngemengen berücksichtigt. Im Zweikulturnutzungssystem wurden daneben auch die Nmin-Mengen nach der Ernte der Erstfrüchte bei der Düngung der Zweitfrucht angerechnet.

Pflanzenschutzmittel wurden praxisüblich eingesetzt. Bei den Varianten, bei denen keine chemische Unkrautregulierung möglich war, wurden mechanische Verfahren eingesetzt (Handund Maschinenhacke). Dies betraf v.a. die Blühmischungen, die Durchwachsene Silphie und den Amarant. 
Tabelle 4: Termine der Aussaat und Ernte sowie Angaben zu Saatstärken und Reihenweiten der Varianten in den Versuchsjahren 2015 und 2016 an den Standorten Reinshof und Schoningen.

\begin{tabular}{|c|c|c|c|c|c|c|c|}
\hline \multirow[t]{2}{*}{ Variante } & \multirow[t]{2}{*}{ Versuchsjahr } & \multicolumn{2}{|c|}{ Datum Aussaat } & \multirow[t]{2}{*}{ Saat- bzw. Pflanzstärke } & \multirow[t]{2}{*}{$\begin{array}{c}\text { Reihen- } \\
\text { abstand (cm) }\end{array}$} & \multicolumn{2}{|c|}{ Datum Ernte } \\
\hline & & Reinshof & Schoningen & & & Reinshof & Schoningen \\
\hline \multirow{2}{*}{ Wickroggen } & 2015 & 29. Sep 14 & 6. Okt 14 * & $110 \mathrm{~kg} \mathrm{ha}^{-1}$ & 17 & 2. Jun 15 & Keine Ernte * \\
\hline & 2016 & 3. Okt 15 & 13. Okt 15 & $120 \mathrm{~kg} \mathrm{ha}^{-1}$ & 17 & 6. Jun 16 & 13. Jun 16 \\
\hline \multirow{2}{*}{$\begin{array}{l}\text { Winterackerbohnen } \\
\text { (WAB) }\end{array}$} & 2015 & 1. Okt 14 & 6. Okt 14 & 25 Körner m-2 & 22 & 2. Jun 15 & 9. Jun 15 \\
\hline & 2016 & 5. Okt 15 & 13. Okt 15 & 25 Körner m-2 & 22 & 6. Jun 16 & 13. Jun 16 \\
\hline \multirow{2}{*}{ Wintertriticale (WT) } & 2015 & 29. Sep 14 & 6. Okt 14 & 375 Körner m-2 & 17 & 2. Jun 15 & 9. Jun 15 \\
\hline & 2016 & 3. Okt 15 & 13. Okt 15 & 375 Körner m² & 17 & 6. Jun 16 & 13. Jun 16 \\
\hline \multirow{2}{*}{ Gemenge WAB / WT } & 2015 & 1. Okt 14 & 6. Okt 14 & WAB 25, WT 281 Körner m-2 & 22 & 2. Jun 15 & 9. Jun 15 \\
\hline & 2016 & 5. Okt 15 & 13. Okt 15 & WAB 25, WT 281 Körner m-2 & 22 & 6. Jun 16 & 13. Jun 16 \\
\hline \multirow{2}{*}{ Sommerackerbohnen } & 2015 & 9. Apr 15 & 25. Mrz 15 & 40 Körner $\mathrm{m}^{-2}$ & 22 & 15. Jul 15 & 16. Jul 15 \\
\hline & 2016 & 16. Mrz 16 & 17. Mrz 16 & 40 Körner m-2 & 22 & 12. Jul 16 & 13. Jul 16 \\
\hline \multirow{2}{*}{$\begin{array}{l}\text { Einjährige Blühmi- } \\
\text { schung }\end{array}$} & 2015 & 8. Mai 15 & 12. Mai 15 & $10 \mathrm{~kg} \mathrm{ha}^{-1}$ & 17 & 5. Okt 15 & 6. Okt 15 \\
\hline & 2016 & 12. Mai 16 & 17. Mai 16 & $10 \mathrm{~kg} \mathrm{ha}^{-1}$ & 17 & 11. Okt 16 & 4. Okt 16 \\
\hline \multirow{2}{*}{ Amarant $(\mathrm{A})$} & 2015 & 18. Mai 15 & 12. Mai 15 & 150 Körner m-2 & 17 & 5. Okt 15 & 6. Okt 15 \\
\hline & 2016 & 10. Mai 16 & 17. Mai 16 & 200 Körner m-2 & 17 & 11. Okt 16 & 4. Okt 16 \\
\hline \multirow{2}{*}{$\begin{array}{l}\text { Mais (M) (Sommer- } \\
\text { hauptfrucht) }\end{array}$} & 2015 & 11. Mai 15 & 12. Mai 15 & 10 Körner m-2 & 75 & 29. Sep 15 & 30. Sep 15 \\
\hline & 2016 & 10. Mai 16 & 11. Mai 16 & 10 Körner m-2 & 75 & 12. Sep 16 & 19. Sep 16 \\
\hline \multirow{2}{*}{$\begin{array}{l}\text { Gemenge Amarant / } \\
\text { Mais }\end{array}$} & 2015 & 11. Mai 15 & 12. Mai 15 & Amarant 75, Mais 10 Körner m-2 & A $37,5, M 75$ & 29. Sep 15 & 30. Sep 15 \\
\hline & 2016 & 10. Mai 16 & 11. Mai 16 & Amarant 100 , Mais 10 Körner $\mathrm{m}^{-2}$ & A $37,5, M 75$ & 12. Sep 16 & 19. Sep 16 \\
\hline \multirow{2}{*}{$\begin{array}{l}\text { Deutsches Weidel- } \\
\text { gras }\end{array}$} & 2015 & 29. Sep 14 & 25. $\operatorname{Mrz} 15^{*}$ & $\begin{array}{c}40 \mathrm{~kg} \mathrm{ha}^{-1} \\
\text { entspricht } 1176 \mathrm{Körner} \mathrm{m}^{-2}\end{array}$ & 17 & $\begin{array}{l}\text { 2. Jun, 5. Aug, } \\
\text { 19. Okt } 15\end{array}$ & \\
\hline & 2016 & & & & & $\begin{array}{c}\text { 6. Jun, 15. Aug, } 7 . \\
\text { Nov } 16\end{array}$ & $\begin{array}{c}\text { 13. Jun, } 18 . \\
\text { Aug, } 31 . \text { Okt } 16\end{array}$ \\
\hline \multirow{2}{*}{$\begin{array}{l}\text { Mehrjährige Blühmi- } \\
\text { schung }\end{array}$} & 2015 & 8. Mai 15 & 12. Mai 15 & $10 \mathrm{~kg} \mathrm{ha}^{-1}$ & 17 & 1. Sep 15 & 24. Aug 15 \\
\hline & 2016 & & & & & 15. Aug 16 & 18. Aug 16 \\
\hline \multirow{2}{*}{$\begin{array}{l}\text { Durchwachsene } \\
\text { Silphie }\end{array}$} & 2015 & 19. Mai 15 & 20. Mai 15 & 4 Pflanzen $\mathrm{m}^{-2}$ & 75 & \multicolumn{2}{|c|}{ keine Ernte im Pflanzjahr } \\
\hline & 2016 & & & & & 31. Aug 16 & 25. Aug 16 \\
\hline \multirow{2}{*}{ Mais (Zweitfrucht) } & 2015 & 8. Jun 15 & 17. Jun 15 & 9 Körner m-2 & 75 & 21. Okt 15 & 5. Nov 15 \\
\hline & 2016 & 10. Jun 16 & 21. Jun 16 & 9 Körner m-2 & 75 & 27. Okt 16 & 31. Okt 16 \\
\hline
\end{tabular}

* Ausfall der Herbstansaat 2014 aufgrund von Schneckenfraß bei den Varianten Wickroggen und Deutsches Weidelgras, daher keine Ernte im Versuchsjahr 2015. 


\subsubsection{Datenerhebung}

\subsubsection{Erhebung der Sprossdaten}

An den Ernteterminen (Tabelle 4) der Feldfrüchte fanden Probenahmen in Miniplots statt. Die Miniplots hatten, abhängig von der Reihenweite, eine Fläche von 0,88 bis $7,5 \mathrm{~m}^{2}$ und wurden auf einem mittleren Beet der Parzellen eingerichtet. Bei den achtreihig gesäten Varianten wurden die inneren sechs Reihen $\left(0,99 \mathrm{~m}^{2}\right)$ auf jeweils einen Meter Länge geerntet, bei den sechsreihig gesäten die inneren vier $\left(0,88 \mathrm{~m}^{2}\right)$. Bei Mais wurden beide Pflanzenreihen auf einer Länge von 5 Metern geerntet $\left(7,5 \mathrm{~m}^{2}\right)$, beim Gemenge aus Amarant und Mais neben den beiden Maisreihen auch die vier Amarantreihen (7,5 $\left.\mathrm{m}^{2}\right)$. Da die Durchwachsene Silphie sehr viele Triebe gebildet hatte, wurden hier trotz des großen Reihenabstands zwei Reihen mit jeweils einem Meter Länge geerntet (1,5 m²). Um die Randeffekte weiter zu minimieren, wurde an den Stirnseiten der Parzellen mindestens ein Meter Beetfläche verworfen. Die Pflanzen wurden mithilfe einer Säge bodennah abgeschnitten. Am Standort Schoningen wurde das Unkraut im ersten Versuchsjahr bei der mehrjährigen Blühmischung und im zweiten Versuchsjahr bei der ein- und mehrjährigen Blühmischung aus der Gesamtprobe aussortiert und gesondert erfasst. Bei allen anderen Varianten war dies nicht notwendig. Durch Wägung (Laborwaage Firma Sartorius, Typ TE 1502 S) und Trocknung bei $105^{\circ} \mathrm{C}$ (Trockenschrank Firma Memmert, Typ ULM800) wurde der Trockensubstanzgehalt (\%) bestimmt und der Trockenmasseertrag (dt TM ha-1) berechnet. Zudem wurden Teilproben zur Bestimmung der Inhaltsstoffe bei $60^{\circ} \mathrm{C}$ getrocknet. Nach dem Vermahlen der Proben (Firma Retsch, Typ ZM, 0,2 mm Maschenweite) und der Einwaage in Zinnkapseln wurden die Gehalte an Kohlenstoff und Stickstoff (\%) mithilfe eines $\mathrm{C} / \mathrm{N}$-Elementaranalysators (Firma Elementar Analysensysteme, Typ Vario EL) ermittelt.

Anhand weiterer, bei $60^{\circ} \mathrm{C}$ getrockneter Teilproben wurde der spezifische Methanertrag $\left(\mathrm{Nm}^{3}\right.$ $\mathrm{CH}_{4} \mathrm{~kg}^{-1}$ OTM) bestimmt. Dazu wurde die Biomasse der Varianten des zweiten Versuchsjahres vom Standort Reinshof genutzt. Bei der mehrjährigen Blühmischung wurde zudem die Biomasse des ersten Versuchsjahres ebenfalls vom Standort Reinshof untersucht, da es hier vom ersten zum zweiten Versuchsjahr zu einer Veränderung in der Artenzusammensetzung kam. Die Analyseergebnisse der spezifischen Methanerträge für die Biomasse vom Standort Reinshof des Versuchsjahres 2016 wurden bei der Berechnung der Methanflächenerträge für beide Standorte und beide Versuchsjahre angenommen. Die Proben setzten sich aus einer Mischung der vier Feldwiederholungen zusammen. Für jede Variante wurde demzufolge eine Probe analysiert. Bei den Gemengen aus Winterackerbohnen und Wintertriticale sowie Amarant und Mais wurden die spezifischen Methanerträge der Reinsaaten genutzt und entsprechend der Artanteile am Gesamttrockenmasseertrag die spezifischen Methanerträge für die Gemenge berechnet. Ebenso, wenn auch nicht mit den Reinsaaten, wurde bei Wickroggen vorgegangen. Hier wurden die Arten Winterwicke und Winterroggen getrennt aufgearbeitet und analysiert.

Zur Bestimmung der spezifischen Methanerträge wurde der „Hohenheimer Biogasertragstest“ (HBT) nach VDI 4630 angewandt. Beim Hohenheimer Biogasertragstest werden 500 mg des Testmaterials zusammen mit einem Impfsubstrat in Glasspritzen (Kolbenprober) eingegeben. Die als Fermenter dienenden und gasdicht verschlossenen Glasspritzen werden unter ständiger Durchmischung in einen Brutschrank gegeben. Über eine Öffnung an der Glasspritze erfolgt die Gasanalyse. Das Probenmaterial wird zudem auf den Gehalt an Trockensubstanz, organischer Trockensubstanz und Asche untersucht (Helffrich \& Oechsner 2003). Jede Probe 
wurde dreifach wiederholt analysiert. Die Temperatur des Brutschranks betrug $37^{\circ} \mathrm{C}$, die gesamte Verweildauer 35 Tage. Die Analysen wurden vom Labor „T\&B - Die Biogasoptimierer $\mathrm{GmbH}$ “ in Tarp durchgeführt.

Aus den spezifischen Methanerträgen und den Trockenmasseerträgen, korrigiert um den Anteil organischer Trockenmasse an der Trockenmasse, wurden die Methanflächenerträge $\left(\mathrm{Nm}^{3}\right.$ $\mathrm{CH}_{4}$ ha $^{-1}$ ) berechnet.

Die Spurenelementanalyse der oberirdischen Biomasse wurde von der Abteilung Sedimentologie und Umweltgeologie der Georg-August-Universität Göttingen mittels Säuretotalaufschluss und optischer Emissionsspektrometrie (ICP-OES) sowie Massenspektrometrie (ICPMS) durchgeführt (Fahlbusch et al. 2018). Insgesamt wurden mit diesem Verfahren 47 Spurenelemente in der Biomasse analysiert. Von diesen werden in den weiteren Ausführungen zwei berücksichtigt - Cobalt und Nickel.

Am Standort Reinshof wurden die Trockenmasseerträge der Zweitfrucht Mais des Versuchsjahres 2016 bei den Varianten Wickroggen + Mais, Wintertriticale + Mais und Gemenge WAB / WT + Mais aufgrund von teilweise verlorengegangener Daten geschätzt. Dazu wurden einmal die vorhandenen Trockenmasseerträge von Mais nach Winterackerbohnen (Winterackerbohnen + Mais) des Versuchsjahres 2016 sowie die Trockenmasseerträge der Zweitfruchtvarianten des Versuchsjahres 2015 genutzt. Dies war möglich, da die Trockenmasseerträge der Zweitfrucht an beiden Standorten im ersten Versuchsjahr kaum eine Abhängigkeit von der Winterzwischenfruchtvariante zeigten. Zudem wurde der Mais als Zweitfrucht in beiden Versuchsjahren nach allen Erstfrüchten auf das gleiche Niveau mit Stickstoff aufgedüngt. Ein von den Erstfrüchten unbeeinflusster Trockenmasseertrag der Zweitfrucht wurde daher auch für das zweite Versuchsjahr angenommen. Am Standort Schoningen waren hingegen die Daten für beide Versuchsjahre vollständig verfügbar.

\subsubsection{Erhebung der Wurzeldaten}

Zur Erfassung der Wurzelmassen wurden auf den Ernteplots Rammkernsonden (Firma Eijkelkamp) mit einem Innendurchmesser von 8,7 cm mithilfe eines Bohrhammers (Firma Makita, Typ MH1400), am Standort Reinshof bis $60 \mathrm{~cm}$ und am Standort Schoningen aufgrund des steinigen Bodens bis $30 \mathrm{~cm}$ Tiefe, in den Boden getrieben. Pro Parzelle erfolgte ein Einstich auf der Pflanzenreihe und ein weiterer Einstich zwischen den Pflanzenreihen. Bei den Gemengen (Winterackerbohnen / Wintertriticale und Amarant / Mais) gab es drei Einstiche pro Parzelle, auf den Reihen der beiden Arten und dazwischen. Die Bodenproben wurden am Standort Reinshof in die Bodenabschnitte 0 bis $30 \mathrm{~cm}$ und 30 bis $60 \mathrm{~cm}$ getrennt. In einer halbautomatischen Wurzelwaschanlage (Firma UP, $1 \mathrm{~mm}$ Maschenweite des Siebs) und nachgeschaltetem Auslesen per Hand wurden die Wurzeln von Boden und Fremdmaterial separiert. Nach der Trocknung (Trockenschrank Firma Memmert, Typ ULM800) und Wägung (Feinwaage Firma Sartorius, Typ LC620 P, $\pm 0,001 \mathrm{~g}$ ) der Wurzeln wurde die Wurzeltrockenmasse je Flächeneinheit ( $\mathrm{dt} \mathrm{TM} \mathrm{ha}^{-1}$ ) berechnet. Dabei wurde ein Mittelwert aus den Beprobungspositionen auf und zwischen den Pflanzenreihen gebildet.

Die bei $60{ }^{\circ} \mathrm{C}$ getrockneten Wurzelproben wurden anschließend vermahlen (Firma Retsch, Typ ZM, 0,2 mm Maschenweite) und entsprechend der oberirdischen Biomasse mit dem C/NElementaranalysator auf die Gehalte an Kohlenstoff und Stickstoff untersucht. 


\subsubsection{Erhebung der Bodendaten}

Nmin-Beprobungen in drei Tiefenstufen $(0-30,30-60$ und $60-90 \mathrm{~cm})$ fanden statt im Winter (Januar 2015 und Januar 2016) und nach der Ernte der Feldfrüchte. Da die Bodenprobenahme für die Bestimmung der Nmin-Mengen und Wassergehalte mittels eines Bohrstabs mit deutlich geringerem Durchmesser als bei der Wurzelprobenahme stattfand, konnten am Standort Schoningen Nmin- und Wassergehaltsproben bis zu den angestrebten $90 \mathrm{~cm}$ (Nmin) bzw. 80 $\mathrm{cm}$ (Wasser) entnommen werden. Die Beprobung im Winter erfolgte im ersten Versuchsjahr auf den Parzellen der Winterzwischenfrüchte und des Deutschen Weidelgrases sowie auf zwei Parzellen, die über Winter als Schwarzbrache vorlagen. Im zweiten Versuchsjahr wurden bei der Winter-Nmin-Beprobung ebenfalls die Winterzwischenfrüchte und zwei Schwarzbrachen beprobt, aber auch die zu diesem Zeitpunkt vollständig etablierten mehrjährigen Varianten. Neben der Nmin-Beprobung im Winter wurde die Menge mineralischen Stickstoffs auch nach der Ernte der Feldfrüchte bestimmt. Die Bodenproben wurden mithilfe einer mobilen Probenahme-Vorrichtung entnommen. Dabei wurde ein Pürckhauer Bohrstab (Firma Nietfeld) mit einem elektrisch betriebenen Schlaghammer (Firma Hilti) in den Boden getrieben und mittels einer Winde (Firma Roller) wieder aus dem Boden gezogen. Die in drei Bodenabschnitte unterteilten Bodenproben wurden in Polyethylen-Beutel verpackt und bis zur Extraktion tiefgefroren. Zur Extraktion des mineralischen Stickstoffs aus den Bodenproben wurden im Labor 100 g Boden mit $250 \mathrm{ml} \mathrm{0,01} \mathrm{M} \mathrm{Calciumchloridlösung} \mathrm{versetzt.} \mathrm{Nach} \mathrm{einstündigem} \mathrm{Schütteln} \mathrm{der}$ Proben im Horizontalschüttler wurden die Proben filtriert. Die Analyse der Extrakte auf die Gehalte an Ammonium $\left(\mathrm{NH}_{4}{ }^{+}\right)$und Nitrat $\left(\mathrm{NO}_{3}{ }^{-}\right)$wurde mit dem AutoAnalyzer 3 (Firma Seal Analytical) vorgenommen. Daneben wurde die Trockenmasse der Bodenproben durch Trocknen bei $105^{\circ} \mathrm{C}$ ermittelt. Unter Berücksichtigung der in Bodengruben bestimmten Rohdichten (1,32 bzw. $1,38 \mathrm{~g} \mathrm{~cm}^{-3}$ in 0-30 cm, 1,45 bzw. $1,60 \mathrm{~g} \mathrm{~cm}^{-3}$ in 30-60 cm und 1,62 bzw. $1,71 \mathrm{~g}$ $\mathrm{cm}^{-3}$ in 60-90 $\mathrm{cm}$ an den Standorten Reinshof und Schoningen) und der Trockenmasse des Bodens wurden die Nmin-Mengen $\left(\mathrm{kg} \mathrm{ha}^{-1}\right)$ errechnet.

Zur Bestimmung der zu Vegetationsbeginn zur Verfügung stehenden und nach der Ernte zurückgebliebenen Menge an pflanzenverfügbarem Wasser, wurden ähnlich der Nmin-Beprobung Bodenproben mit einem Pürckhauer Bohrstab bis $80 \mathrm{~cm}$ Tiefe auf den Parzellen entnommen. In $10 \mathrm{~cm}$ Bodenabschnitte eingeteilt, wurden die Bodenproben in mit Deckeln versehene Kunststoffbehälter gefüllt, feucht und getrocknet $\left(105^{\circ} \mathrm{C}\right)$ gewogen und daraus der gravimetrische Bodenwassergehalt bestimmt. Mittels der an den Standorten Reinshof und Schoningen angefertigten pF-Kurven konnten mit den Bodenwassergehalten die Mengen an pflanzenverfügbarem Wasser berechnet werden.

\subsection{Umfrage zur Erhebung der Akzeptanz des Energiepflanzenanbaus in der Bevölke- rung}

Zur Erfassung der Akzeptanz des Energiepflanzenanbaus in der Bevölkerung wurde von Ende Juli bis Anfang Dezember 2017 eine Online-Umfrage mithilfe des von der Gesellschaft für wissenschaftliche Datenverarbeitung mbH Göttingen (GWDG) zur Verfügung gestellten Tools „LimeSurvey“ durchgeführt. Bei dem Fragebogen ging es um die Einschätzung des Energiepflanzenanbaus zur Biogaserzeugung und speziell um die in der vorliegenden Arbeit untersuchten und daher vorgeschlagenen Varianten. Der Fragebogen war so konstruiert, dass geschlossene Fragen gestellt wurden aber es zusätzlich auch die Möglichkeit gab, ausführlich in 
einem Freitextfeld zu antworten. Während die Antworten für die geschlossenen Fragen Pflichtangaben waren, ohne die ein Fortfahren nicht möglich gewesen wäre, waren die Freitextangaben freiwillig. Der erste von insgesamt drei Fragenkomplexen umfasste die Beurteilung regenerativer Energien und dabei besonders der Anbau von Energiepflanzen zur Biogaserzeugung. Als Antwortmöglichkeiten wurden 7-stufige Likert-Skalen für die Einschätzung von sehr schlecht bis sehr gut angeboten. Beim zweiten Fragenkomplex konnten die Teilnehmer die empfundene Landschaftsästhetik der vorgeschlagenen und anhand von Fotos sichtbar gemachten Pflanzenvarianten bewerten (7-stufige Likert-Skala von gefällt mir gar nicht bis gefällt mir sehr gut) sowie die Akzeptanz des Anbaus, mit den Antwortmöglichkeiten „Ja“, „Nein“ oder „Vielleicht“, beurteilen. Der dritte Fragenkomplex beinhaltete demographische Angaben.

Tabelle 5: Geschlechtsverteilung, Altersverteilung, Bildungsabschluss sowie Angabe zur Naturverbundenheit der Umfrageteilnehmer $(n=446)$.

\begin{tabular}{lc}
\hline Geschlecht & $\%$ \\
\hline männlich & 53,0 \\
weiblich & 45,7 \\
keine Angabe & 1,4 \\
\hline Alter & \\
\hline$\leq 20$ Jahre & 2,1 \\
21-30 Jahre & 29,2 \\
31-40 Jahre & 21,0 \\
41-50 Jahre & 18,3 \\
$51-60$ Jahre & 18,5 \\
61-70 Jahre & 9,4 \\
71-80 Jahre & 1,8 \\
$\geq 81$ Jahre & 0,5 \\
\hline Bildungsabschluss & \\
\hline Kein Schulabschluss & 0,0 \\
Grund-/Hauptschulabschluss & 0,5 \\
Realschule (Mittlere Reife) & 1,8 \\
Gymnasium (Abitur) & 7,8 \\
Abgeschlossene Berufsausbildung & 5,0 \\
Fachhochschulabschluss & 6,6 \\
Hochschule (Bachelor) & 11,0 \\
Hochschule (Diplom, Master, Magister) & 40,0 \\
Hochschule (Promotion) & 25,3 \\
Sonstiges & 2,1 \\
keine Antwort & 0,0 \\
\hline Naturverbundenheit & 9,1 \\
\hline Nein & 67,6 \\
Ja, beruflich & 14,8 \\
Ja, Hobby & 8,4 \\
Sonstiges & 0,0 \\
keine Antwort & \\
\hline & \\
\hline
\end{tabular}


Die Teilnehmer wurden per Email über verschiedene universitäre und außeruniversitäre Verteiler zur Umfrage eingeladen. Außerdem wurden die Teilnehmer in der Einladungs-Email aufgefordert, weitere Personen einzuladen. Von den 575 eingegangenen Fragebögen waren 446 vollständig und damit auswertbar. Die Differenz zwischen eingegangenen und auswertbaren Fragebögen entstand durch das Zwischenspeichern begonnener aber nicht abgeschlossener Fragebögen im System.

Die quantitativen Daten wurden mit Analyseverfahren der deskriptiven Statistik ausgewertet. Die qualitativen Daten, d.h. die Antworten auf die offenen Fragen in den Freitextfeldern, wurden mit inhaltsanalytischen Verfahren ausgewertet. Die Methode, die hier zum Einsatz kam, war die Frequenzanalyse nach Mayring (2010). Dabei wird zunächst ein Kategoriensystem aufgestellt, deren Häufigkeiten untersucht werden sollen. Für die Entwicklung von Kategorien sind deduktive (theoriegeleitete) oder induktive Verfahren möglich. In der vorliegenden Untersuchung kam der induktive Ansatz zur Anwendung, bei dem die Kategorien in einem Verallgemeinerungsprozess aus dem zu untersuchenden Material direkt abgeleitet werden. Schließlich wird das Material mithilfe des Kategoriensystems durchgearbeitet und das Auftreten der Kategorien aufgezeichnet. Die Häufigkeiten der einzelnen Kategorien werden dargestellt (Mayring 2010).

Eine Übersicht über die demografischen Angaben der Umfrageteilnehmer sowie die berufliche und interessengeleitete Einordnung ist in Tabelle 5 dargestellt.

\subsection{Deckungsbeitragsberechnung}

Aus den im Feldversuch erhobenen Daten wurde eine ökonomische Bewertung (Deckungsbeitrag I) der Feldfrüchte vorgenommen. In die Deckungsbeitragsberechnungen gingen die Leistungen und variablen Kosten ein. Als Leistung wurden der Erlös aus der Biomasse sowie der Düngewert des nach der Vergärung der Biomasse entstandenen Gärrests angerechnet. Zur Quantifizierung der variablen Kosten, die beim Anbau der verschiedenen Pflanzenvarianten entstehen, wurden Daten des KTBL $(2009,2013)$ herangezogen. Die Kosten jeder Variante wurden dabei je nach durchgeführten Arbeitsgängen und Maßnahmen individuell berechnet. Bei den Varianten des Zweikulturnutzungssystems wurden die Leistungen und Kosten der Erst- und Zweitfrüchte berücksichtigt. Bei der Durchwachsenen Silphie wurden das Anpflanzjahr, 15 Jahre Nutzungsdauer und der Umbruch im letzten Jahr in die Kalkulation einbezogen. Bei der mehrjährigen Blühmischung wurden fünf, beim Deutschen Weidelgras vier Nutzungsjahre mit Aussaat im ersten und Umbruch im letzten Jahr angenommen. Die höheren Kosten des ersten und letzten Jahres wurden dabei auf die Nutzungsjahre aufgeteilt. Die angegebenen Deckungsbeiträge wurden berechnet indem die Summe aller Kosten über die Jahre des Anbaus (Anpflanz- bzw. Aussaatjahr bis Umbruch) der Summe der Leistungen über die gleiche Zeit abgezogen wurden. Die jährlichen Deckungsbeiträge beinhalten also die Kosten der Anpflanzung bzw. Aussaat sowie des Umbruchs, entsprechend der Nutzungsjahre anteilig. Eine beispielhafte Berechnung der Deckungsbeiträge findet sich im Anhang (Tabelle A3). 


\subsection{Statistik}

Die statistischen Analysen der Daten wurden mit der Open-Source-Software R (Version 3.5.3., $R$ Core Team 2019) durchgeführt. Es wurde ein lineares gemischtes Modell aufgestellt, das sowohl feste als auch zufällige Effekte berücksichtigt (Funktion „Imer“ aus dem R-Paket „Ime4“, Bates et al. 2019). Bei den Kenngrößen Spross-Trockenmasseertrag, Trockensubstanzgehalt, N-Akkumulation im Spross, Methanflächenertrag, Cobalt- und Nickelgehalt, Wurzel-Trockenmasseertrag, C/N-Verhältnis der Wurzeln sowie N-Akkumulation in den Wurzeln gingen neben den Varianten auch die Versuchsjahre als feste Faktoren in das Modell ein. Bei der Analyse der Nmin-Werte wurde der Jahreseffekt nicht in das Modell aufgenommen. Als zufällig wurden die Effekte angenommen, die durch das Versuchsdesign (vertikale und horizontale Blockbildungsrichtung, Lateinisches Quadrat) zustande kamen. Die Standorte Reinshof und Schoningen wurden gesondert ausgewertet. Die Modelvoraussetzungen, Varianzhomogenität und Normalverteilung der Residuen der einzelnen Modelle wurden visuell überprüft. War keine Normalverteilung oder Varianzhomogenität gegeben, wurden die Daten transformiert (Logarithmus oder Quadratwurzel).

Um die Signifikanz der festen Faktoren zu testen, wurde eine Varianzanalyse (ANOVA) durchgeführt. Post-hoc-Tests erfolgten mit der Funktion „emmeans“ des gleichnamigen R-Pakets (Lenth et al. 2019). Dabei wurden die Unterschiede zwischen den Faktorstufen des Faktors Variante mittels multipler paarweiser Vergleiche nach der Tukey-Methode berechnet. Vergleiche zwischen den Jahren innerhalb einer Variante erfolgten mittels t-Test. Als Signifikanzniveau wurde $p \leq 0,05$ verwendet. Zudem wurden lineare Zusammenhänge zwischen ausgewählten Parametern über den Korrelationskoeffizienten ( $r$ ) nach Pearson berechnet.

\subsection{Berechnung des Index der relativen Anbauwürdigkeit (IrA)}

Zur Bewertung der Feldfrüchte wurde der Index der relativen Anbauwürdigkeit (IrA) (Menke 2011) erweitert. In der vorliegenden Arbeit setzt sich der Index IrA zusammen aus den Teilindizes:

- Spurenelementgehalt (Cobalt und Nickel)

- Trockenmasseertrag (oberirdisch)

- Methanflächenertrag

- Trockensubstanzgehalt

- Nmin im Winter und nach der Ernte

- Wurzel-Trockenmasse

- Bodenwasser

- Soziale Aspekte (Akzeptanz)

- Ökonomische Aspekte (Deckungsbeitrag)

\subsubsection{Berechnung der Teilindizes des Index IrA}

Die Teilindizes wurden berechnet aus den Daten der erhobenen Parameter bzw. Kenngrößen. Dabei beschreibt der Teilindex den Wert einer Kenngröße der betrachteten Variante im Verhältnis zum mittleren Wert der Kenngröße aller geprüften Varianten (Menke 2011). 
Zur Berechnung der Teilindex-Werte für Spurenelementgehalt, Trockenmasseertrag, Methanflächenertrag, Trockensubstanzgehalt, Wurzel-Trockenmasse, Bodenwasser, Akzeptanz und Deckungsbeitrag, also der Kenngrößen, bei denen höhere Werte positiv bewertet werden, wurde die allgemeine Formel angewandt:

$$
\text { Teilindex } x=\frac{x}{\bar{x}}-1
$$

wobei $\mathrm{X}=$ Wert für die Kenngröße $\mathrm{X}$ einer Variante in der Einheit der Kenngröße

$\bar{X}=\quad$ arithmetischer Mittelwert für die Kenngröße $X$ aller Varianten in der Einheit der Kenngröße

Die Werte für die so berechneten Teilindizes sind dimensionslos und liegen, bis auf den Teilindex ökonomische Bewertung, in einem Wertebereich von -1 bis $+\infty$. Der in den Teilindex ökonomische Bewertung eingehende Deckungsbeitrag kann sowohl positive als auch negative Werte annehmen, sodass der Teilindex ökonomische Bewertung einen Wertebereich von $-\infty$ bis $+\infty$ hat.

Für die Berechnung der Teilindex-Werte wurde die allgemeine Formel an die jeweilige Kenngröße angepasst. Am Beispiel des Teilindex Trockenmasseertrag lautete die Formel daher:

$$
\text { Teilindex TM-Ertrag }=\frac{\mathrm{Y}}{\overline{\mathrm{Y}}}-1
$$

wobei $\mathrm{Y}=$ Trockenmasse-Ertrag einer Variante $(\mathrm{dt}$ TM ha-1)

$\bar{Y}=\quad$ arithmetischer Mittelwert der Trockenmasseerträge aller Varianten (dt TM ha ${ }^{-1}$ )

Der Teilindex-Wert einer Variante für eine bestimmte Kenngröße errechnet sich demzufolge daraus, dass der Wert einer Kenngröße der betreffenden Variante ins Verhältnis gesetzt wird zum Mittelwert der Kenngrößen aller Varianten. Um einen Indexwert zu erhalten wird der Quotient subtrahiert um 1. Ist der Wert der Kenngröße einer bestimmten Variante oberhalb des Mittelwerts aller Varianten, ergibt sich ein positiver Teilindex-Wert, ist der Wert der Variante unterhalb des Mittelwerts, ergibt sich entsprechend ein negativer.

Für die Berechnung des Teilindex Spurenelemente wurden die Mengen (in $\mathrm{mg} \mathrm{ha}^{-1}$ ) an Cobalt (Co) und Nickel (Ni) herangezogen, die in einer Vegetationsperiode bei den untersuchten Varianten vom Feld abgefahren wurden. Dazu wurden die Co- und Ni-Gehalte der Feldfrüchte mit den Trockenmasseerträgen multipliziert. Bei den Varianten des Zweikulturnutzungssystems geht die Summe aus Erst- und Zweitfrucht in die Berechnung ein. Der Teilindex Trockenmasseertrag bildet den Trockenmasseertrag der oberirdischen Biomasse (in dt TM ha ${ }^{-1}$ ) ab. Bei mehrfachen Ernten in einer Vegetationsperiode, wie es beim Zweikulturnutzungssystem sowie dem Deutschen Weidelgras der Fall ist, geht der Gesamtertrag über alle Ernten ein. Ebenso ist der Teilindex Methan, der den Methanflächenertrag (in $\mathrm{Nm}^{3} \mathrm{CH}_{4}$ ha-1) abbildet, aufgebaut. Der Teilindex Trockensubstanzgehalt gibt den Trockensubstanzgehalt (in \%) bei der Ernte der Feldfrüchte wieder. Bei den Varianten des Zweikulturnutzungssystems wird sowohl der Trockensubstanzgehalt der Erst- als auch der Zweitfrucht in Abhängigkeit der Anteile 
am Gesamtertrag berücksichtigt. Gleiches gilt für das mehrschnittige Deutsche Weidelgras. In den Teilindex Wurzel-Trockenmasse gehen die ermittelten Wurzel-Trockenmasseerträge (in dt TM ha-1) zum Zeitpunkt der Ernte der oberirdischen Biomasse ein. Beim Zweikulturnutzungssystem wurden die Wurzel-Trockenmassen der Erst- und Zweitfrucht aufsummiert. Auch bei den mehrjährigen Varianten wurde, wie bei den einjährigen Varianten, in jedem der beiden Versuchsjahre der Trockenmasseertrag der Wurzeln zum Zeitpunkt der Ernte bestimmt und einkalkuliert. Für die Wurzeluntersuchung gingen beim Deutschen Weidelgras am Standort Reinshof in beiden Versuchsjahren und am Standort Schoningen im zweiten Versuchsjahr der jeweils letzte Schnitt ein, da dies einen möglichen Umbruchstermin darstellen könnte zu dem die Wurzelmassen relevant sind.

Um die pflanzenverfügbaren Mengen an Bodenwasser (in $\mathrm{mm}$ ) zu den beiden relevanten Zeitpunkten, Frühjahr sowie Sommer/Herbst zu berücksichtigen, wurden beim Teilindex Bodenwasser zwei separate Teilindizes berechnet. Der Teilindex Bodenwasser (f) spiegelt den Zustand des Bodenwassers im Frühjahr zu Vegetationsbeginn bzw. zur Aussaat der Sommerungen wider, der Teilindex Bodenwasser (s/h) wiederum den Zustand nach der Ernte der Feldfrüchte im Sommer bzw. Herbst. Der Mittelwert der beiden Teilindizes geht als Teilindex Bodenwasser in den Gesamtindex IrA ein.

Der Teilindex Ökonomische Aspekte bildet die ökonomische Bewertung der Varianten in Form von Deckungsbeiträgen (in $€$ ha $^{-1}$ ) ab. Dabei wurden die Leistungen und Kosten der gesamten Vegetationsperiode berücksichtigt. Der Teilindex Soziale Aspekte wurde aus den Daten der Umfrage und dabei speziell aus der Befürwortung (Akzeptanz) der Pflanzenvarianten (in \%) berechnet.

Für die Berechnung des Teilindex Nmin wurde die Formel so angepasst, dass ein niedrigerer Wert der einzelnen Variante im Vergleich zum Mittelwert aller Varianten zu positiven Indexwerten führt und höhere zu negativen (Menke 2011). Die Formel lautet:

$$
\text { Teilindex Nmin }=\frac{\overline{\mathrm{N}} \min -\mathrm{Nmin}}{\overline{\mathrm{N}} \min }
$$

wobei $\quad \mathrm{Nmin}=\mathrm{Nmin}-$ Wert einer Variante $\left(\mathrm{kg} \mathrm{N} \mathrm{ha}^{-1}\right)$

$\overline{\mathrm{N}} \min =$ arithmetischer Mittelwert der Nmin-Werte aller Varianten $\left(\mathrm{kg} \mathrm{N} \mathrm{ha}^{-1}\right)$

Die Werte für die so berechneten Teilindizes sind ebenfalls dimensionslos und liegen in einem Wertebereich von $-\infty$ bis +1 .

In die Berechnung des Teilindex Nmin gehen sowohl die Nmin-Mengen im Winter als auch nach der Ernte im Sommer bzw. Herbst ein. Um diese beiden Zeitpunkte im Teilindex Nmin zu berücksichtigen, wurde für jeden Zeitpunkt, entsprechend der beschriebenen Vorgehensweise beim Teilindex Bodenwasser, ein separater Teilindex gebildet: Teilindex Nmin (w) für den Winter-Nmin (Nmin-Werte Anfang Januar des jeweiligen Versuchsjahres) und Teilindex Nmin (s/h) für den Zeitpunkt nach der Ernte der Varianten im Sommer bzw. Herbst. Um schließlich einen Wert für den Teilindex Nmin zu erhalten, wurde ein Mittelwert aus den Teilindizes Nmin (w) und Nmin (s/h) gebildet. Für die Varianten, die im Frühjahr nach Schwarzbrache ausgesät 
wurden, wurde der Nmin-Wert der Schwarzbrache im Winter bei der Berechnung des Teilindex Nmin (w) verwendet. Bei den Varianten des Zweikulturnutzungssystems gingen mit der beschriebenen Vorgehensweise sowohl die Erst- (Teilindex Nmin (w)) als auch die Zweitfrucht (Teilindex Nmin(s/h)) in die Berechnung ein. Die Nmin-Mengen im Boden nach der Ernte der Erstfrüchte wurden dabei nicht berücksichtigt.

\subsubsection{Berechnung des Gesamtindex IrA}

Der Gesamtindex IrA ergibt sich aus der Addition der Teilindizes:

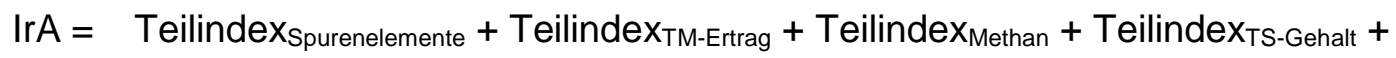

$$
\begin{aligned}
& \text { TeilindeX } X_{\mathrm{Nmin}}+\text { TeilindeX } \text { Wurzeltrockenmasse }_{+} \text {TeilindeX }_{\text {Bodenwasser }}+\text { TeilindeX } X_{\text {Soziale Aspekte }}+ \\
& \text { TeillindeXökonomische Aspekte }
\end{aligned}
$$

Die einzelnen Teilindizes wurden auf der Grundlage verschiedener Daten berechnet. Um den Gesamtindex IrA aufgrund der daraus entstandenen Variation in den absoluten Werten der Teilindizes nicht zu verzerren, wurden die Teilindexwerte standardisiert und erst danach zum Gesamtindex IrA aufaddiert. Dabei gingen alle Teilindizes unter gleicher Gewichtung in den Index IrA ein. Zur Standardisierung wurde die R-Funktion „scale“ (R-Paket „base“, R Core Team 2019) genutzt.

Die Formel der Standardisierung lautet:

$$
\mathrm{Y}=(\mathrm{X}-\mu) / \sigma
$$

wobei $Y=$ Standardisierter Teilindexwert $\mathrm{X}=$ Teilindexwert $\mu=$ Mittelwert aller Teilindexwerte $\sigma=$ Standardabweichung aller Teilindexwerte

Bei der Prozedur wird jeder einzelne Teilindexwert vom Mittelwert aller Teilindexwerte abgezogen und die Differenz durch die Standardabweichung aller Teilindexwerte geteilt. Der Mittelwert der standardisierten Teilindexwerte ist schließlich null und die Standardabweichung eins. Damit ist eine Vergleichbarkeit der Werte gegeben. 


\section{$3 \quad$ Ergebnisse}

\subsection{Sprossdaten}

Signifikante Effekte der Variante wurden an beiden Standorten für den Trockenmasseertrag, den Trockensubstanzgehalt, die N-Akkumulation in der oberirdischen Biomasse sowie hinsichtlich der Cobalt- und Nickelgehalte gefunden (Tabelle 6). Signifikant beeinflusst wurden diese Kenngrößen ebenfalls von der Interaktion aus Variante und Jahr. Signifikante Jahreseffekte gab es hingegen nicht für alle Parameter. An beiden Standorten hatte das Jahr einen hoch signifikanten Einfluss auf den Trockensubstanzgehalt, am Standort Reinshof auch auf den Trockenmasse- und Methanflächenertrag und am Standort Schoningen auf den Cobaltgehalt.

Tabelle 6: Ergebnisse der statistischen Analyse (ANOVA) der festen Effekte Variante und Jahr sowie deren Interaktion im linearen gemischten Modell für die erfassten Sprossdaten (Trockenmasseertrag, Trockensubstanzgehalt, N-Akkumulation, Methanflächenertrag, Cobalt- und Nickelgehalt) an den Standorten Reinshof und Schoningen.

\begin{tabular}{|c|c|c|c|c|c|c|}
\hline & \multicolumn{3}{|c|}{ Reinshof } & \multicolumn{3}{|c|}{ Schoningen } \\
\hline & F-Wert & $\operatorname{Pr}>\mathrm{F}$ & & F-Wert & $\operatorname{Pr}>\mathrm{F}$ & \\
\hline $\begin{array}{l}\text { Trockenmasseertrag } \\
\text { Variante }\end{array}$ & 96,51 & $<0,0001$ & $\star \star \star *$ & 77,28 & $<0,0001$ & $\star * \star$ \\
\hline Jahr & 49,69 & $<0,0001$ & $\star \star * *$ & 0,24 & 0.6296 & n.s. \\
\hline Variante $\mathrm{x}$ Jahr & 4,54 & $<0,0001$ & $\star \star \star *$ & 2,58 & 0.0171 & * \\
\hline $\begin{array}{l}\text { Trockensubstanzgehal } \\
\text { Variante } \\
\text { Jahr } \\
\text { Variante } \mathrm{x} \text { Jahr }\end{array}$ & $\begin{array}{l}188,18 \\
19,51 \\
10,71\end{array}$ & $\begin{array}{l}<0,0001 \\
<0,0001 \\
<0,0001\end{array}$ & $\begin{array}{l}* * * \\
* * * \\
* * *\end{array}$ & $\begin{array}{l}104,75 \\
95,54 \\
28,71\end{array}$ & $\begin{array}{l}<0,0001 \\
<0,0001 \\
<0,0001\end{array}$ & $\begin{array}{l}* \star * \\
\star * * \\
* \star *\end{array}$ \\
\hline $\begin{array}{l}\text { N-Akkumulation } \\
\text { Variante } \\
\text { Jahr } \\
\text { Variante } \mathrm{x} \text { Jahr }\end{array}$ & $\begin{array}{l}70,87 \\
1,08 \\
2,27\end{array}$ & $\begin{array}{l}<0,0001 \\
0.3027 \\
0.0238\end{array}$ & $\begin{array}{l}* * * \\
\text { n.s. } \\
*\end{array}$ & $\begin{array}{l}51,53 \\
0,01 \\
3,33\end{array}$ & $\begin{array}{l}<0,0001 \\
0,9124 \\
0,0031\end{array}$ & $\begin{array}{l}* * * \\
\text { n.s. }\end{array}$ \\
\hline $\begin{array}{l}\text { Methanflächenertrag } \\
\text { Variante } \\
\text { Jahr } \\
\text { Variante x Jahr }\end{array}$ & $\begin{array}{l}169,35 \\
67,33 \\
6,89\end{array}$ & $\begin{array}{l}<0,0001 \\
<0,0001 \\
<0,0001\end{array}$ & $\begin{array}{l}* * * \\
* * * \\
* * *\end{array}$ & $\begin{array}{l}118,70 \\
0,16 \\
2,41\end{array}$ & $\begin{array}{l}<0,0001 \\
0,6877 \\
0,0251\end{array}$ & $\begin{array}{l}* * * \\
\text { n.s. } \\
*\end{array}$ \\
\hline $\begin{array}{l}\text { Cobaltgehalt } \\
\text { Variante } \\
\text { Jahr } \\
\text { Variante x Jahr }\end{array}$ & $\begin{array}{l}157,64 \\
0,07 \\
2,18\end{array}$ & $\begin{array}{l}<0,0001 \\
0,7939 \\
0,0305\end{array}$ & $\begin{array}{l}* * * \\
\text { n.s. } \\
*\end{array}$ & $\begin{array}{l}42,83 \\
5,08 \\
2,73\end{array}$ & $\begin{array}{l}<0,0001 \\
0,0277 \\
0,0119\end{array}$ & $\begin{array}{l}* * * \\
* \\
*\end{array}$ \\
\hline $\begin{array}{l}\text { Nickelgehalt } \\
\text { Variante } \\
\text { Jahr } \\
\text { Variante x Jahr }\end{array}$ & $\begin{array}{l}153,25 \\
0,50 \\
3,47\end{array}$ & $\begin{array}{l}<0,0001 \\
0,4808 \\
0,0011\end{array}$ & $\begin{array}{l}* * * \\
\text { n.s. } \\
* *\end{array}$ & $\begin{array}{l}28,83 \\
0,52 \\
2,39\end{array}$ & $\begin{array}{l}<0,0001 \\
0,4755 \\
0,0268\end{array}$ & $\begin{array}{l}* * * \\
\text { n.s. } \\
*\end{array}$ \\
\hline
\end{tabular}

Signifikanzniveau: ${ }^{* * *} p \leq 0,001 ;{ }^{* *} p \leq 0,01 ;{ }^{*} p \leq 0,05 ;$ n.s. $=$ nicht signifikant. 


\subsubsection{Spross-Trockenmasseertrag}

\section{Reinshof}

Im ersten Versuchsjahr konnten am Standort Reinshof Trockenmasseerträge zwischen $92 \mathrm{dt}$ $\mathrm{TM} \mathrm{ha}^{-1}$ bei den Sommerackerbohnen und $245 \mathrm{dt} \mathrm{TM} \mathrm{ha}^{-1}$ beim Zweikulturnutzungssystem aus Wickroggen und nachfolgendem Mais erzielt werden (Abbildung 4). Es konnten zwei Gruppen ausgemacht werden, bei denen sich die Trockenmasseerträge signifikant voneinander unterschieden. Einmal waren es die Varianten des Zweikulturnutzungssystems, Mais als Sommerhauptfrucht, das Gemenge aus Amarant und Mais und das Deutsche Weidelgras mit Erträgen zwischen 203 und 245 dt TM ha-1. Zum anderen die Sommerackerbohnen, die ein- und mehrjährige Blühmischung sowie der Amarant. Mit Trockenmasseerträge von 92 bis $133 \mathrm{dt} \mathrm{ha}^{-1}$ lagen diese deutlich darunter. Beim dreischnittigen Deutschen Weidelgras machte der erste Schnitt in diesem Versuchsjahr mit $106 \mathrm{dt} \mathrm{TM} \mathrm{ha}^{-1}$ etwa die Hälfte des Gesamtertrags aus, der zweite und dritte Schnitt mit jeweils nahezu $50 \mathrm{dt} \mathrm{TM} \mathrm{ha-1}$ ein Viertel (Daten nicht dargestellt).

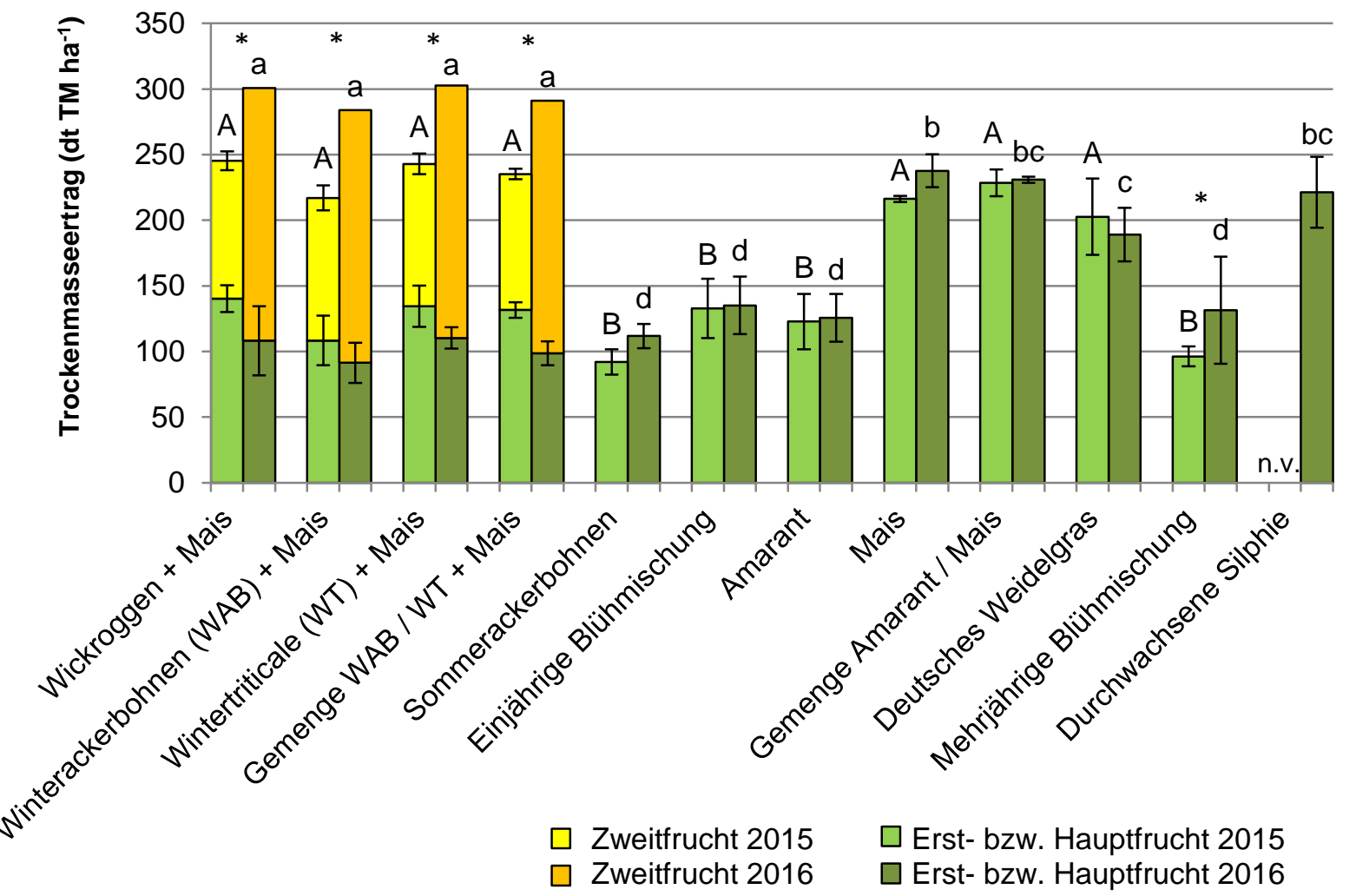

Abbildung 4: Trockenmasseerträge in den Versuchsjahren 2015 und 2016 am Standort Reinshof. Dargestellt sind die Mittelwerte \pm Standardabweichung. Varianten mit gleichen Großbuchstaben (2015) bzw. gleichen Kleinbuchstaben (2016) sind nicht signifikant verschieden, $p<0,05$ (Tukey-Test), ${ }^{*}$ kennzeichnen signifikante Unterschiede zwischen den Versuchsjahren innerhalb einer Variante, $p<0,05$ (t-Test). Bei gestapelten Säulen beziehen sich die Angaben auf die Höhe der Gesamtsäule. n.v. = Wert nicht verfügbar. Keine Angabe der Standardabweichung bei Mais als Zweitfrucht im Versuchsjahr 2016 aufgrund geschätzter Werte (Abschnitt 2.1.5.1).

Im zweiten Versuchsjahr lag das Ertragsniveau bei nahezu allen Varianten höher als im ersten Versuchsjahr. Es bewegte sich zwischen $112 \mathrm{dt} \mathrm{TM} \mathrm{ha-1} \mathrm{bei} \mathrm{den} \mathrm{Sommerackerbohnen} \mathrm{und}$ 302 dt TM ha-1 beim Zweikulturnutzungssystem aus Wintertriticale und nachfolgendem Mais. Mit einem ebenfalls beachtlichen Trockenmasseertrag von nahezu $240 \mathrm{dt} \mathrm{ha}^{-1}$ unterschied sich Mais als Sommerhauptfrucht signifikant von den Varianten des Zweikulturnutzungssystems. Das Gemenge aus Amarant und Mais und auch die Durchwachsene Silphie lagen auf einem 
vergleichbaren Niveau mit Mais. Etwas schwächer, aber nicht signifikant verschieden von den Varianten Gemenge Amarant/Mais und Durchwachsene Silphie, lag das Deutsche Weidelgras mit 190 dt TM ha-1. Wie auch der Gesamtertrag im zweiten Versuchsjahr, unterschieden sich beim Deutschen Weidelgras auch die Trockenmasseerträge der drei Schnitte im Vergleich zum ersten Versuchsjahr. So war der Trockenmasseertrag beim ersten Schnitt mit etwa $90 \mathrm{dt}$ $\mathrm{TM} \mathrm{ha}^{-1}$ geringer, der zweite Schnitt mit $60 \mathrm{dt} \mathrm{TM} \mathrm{ha}^{-1}$ höher und der dritte Schnitt mit $40 \mathrm{dt}$ TM ha $^{-1}$ wiederum geringer als im ersten Versuchsjahr (Daten nicht dargestellt).

Wie im ersten Versuchsjahr bildeten auch im zweiten Versuchsjahr die Varianten Sommerackerbohnen, ein- und mehrjährige Blühmischung sowie Amarant eine Gruppe mit signifikant niedrigeren Trockenmasseerträgen als alle anderen Varianten (Abbildung 4).

Die Trockenmasseerträge von Mais und dem Gemenge aus Amarant und Mais unterschieden sich in beiden Versuchsjahren nicht voneinander. Mit $2 \%$ im ersten und $5 \%$ im zweiten Versuchsjahr hatte der Amarant nur einen geringen Beitrag am Gesamtertrag des Gemenges (Daten nicht dargestellt). Abbildung 5a zeigt den Pflanzenbestand des Gemenges aus Amarant und Mais im Hauptversuch. Der Trockenmasseertrag der im zweiten Versuchsjahr im Rahmen des Amarant-Sorten-Zusatzversuchs zusätzlich angebauten Amarantsorte Pastewny unterschied sich mit $133 \mathrm{dt} \mathrm{TM} \mathrm{ha-1} \mathrm{(Daten} \mathrm{nicht} \mathrm{dargestellt)} \mathrm{nur} \mathrm{wenig} \mathrm{vom} \mathrm{Amarant} \mathrm{im} \mathrm{Haupt-}$ versuch mit $126 \mathrm{dt} \mathrm{TM} \mathrm{ha-1.} \mathrm{Im} \mathrm{Gemenge} \mathrm{mit} \mathrm{Mais} \mathrm{konnte} \mathrm{sich} \mathrm{die} \mathrm{Amarantsorte} \mathrm{Pastewny} \mathrm{mit}$ einem Anteil von $7 \%$ (Daten nicht dargestellt, Abbildung 5b) am Gesamttrockenmasseertrag des Gemenges ebenso wenig durchsetzen wie der Amarant im Hauptversuch, sodass sich auch der Gesamttrockenmasseertrag zwischen den Gemengen der beiden Amarantsorten nicht unterschied.
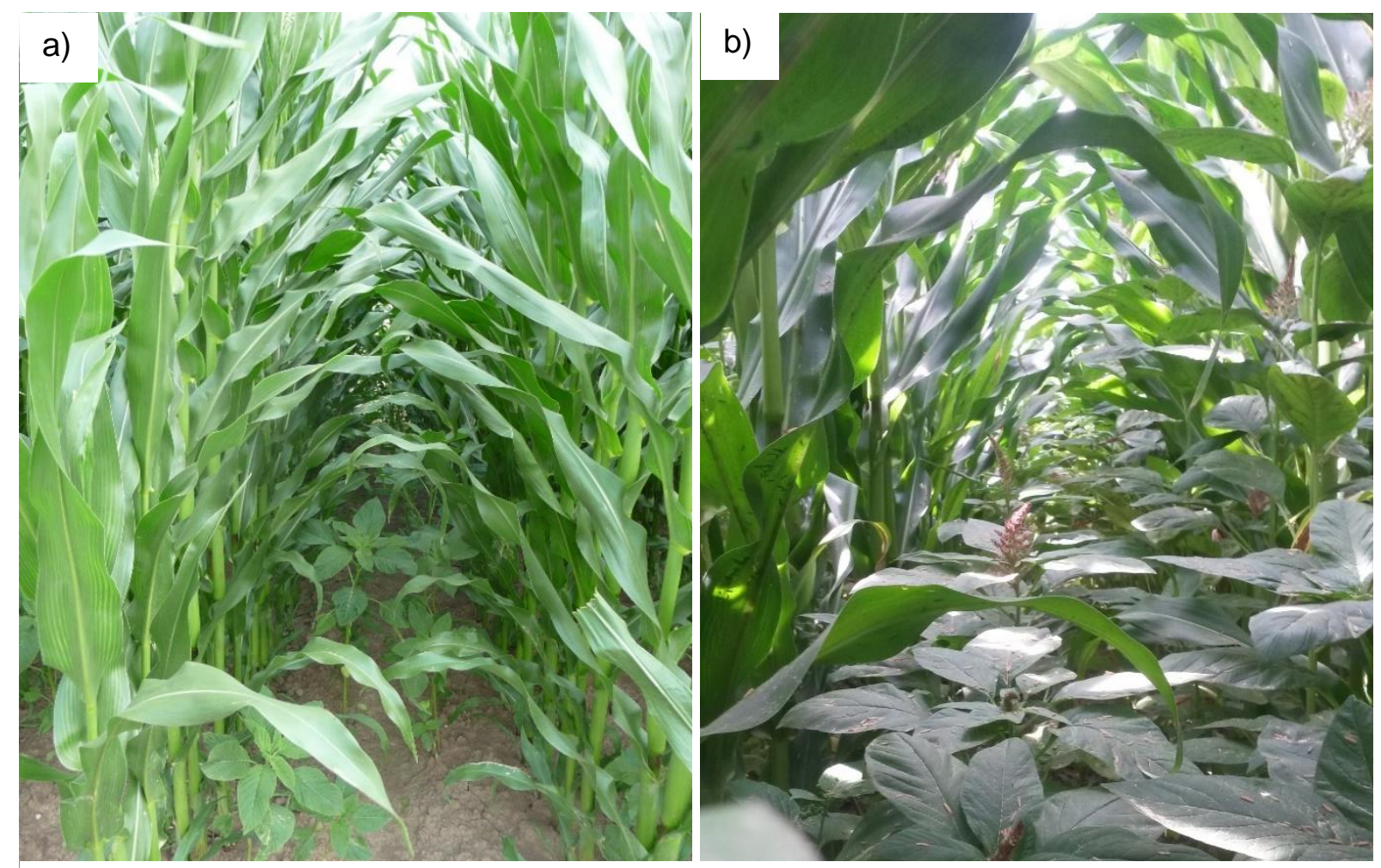

Abbildung 5: Pflanzenbestände des Gemenges aus Amarant und Mais a) am Standort Reinshof im Versuchsjahr 2015 am 23. Juli 2015 (Hauptversuch mit Amarantsorte Bärnkrafft) und b) am Standort Reinshof im Versuchsjahr 2016 am 16. Juli 2016 (Amarant-Sorten-Zusatzversuch mit Amarantsorte Pastewny). 
Signifikante Unterschiede zwischen den Versuchsjahren gab es am Standort Reinshof bei den Varianten des Zweikulturnutzungssystems und der mehrjährigen Blühmischung, wobei das zweite Versuchsjahr zu höheren Trockenmasseerträgen führte als das erste (Abbildung 4). Bei den Varianten des Zweikulturnutzungssystems waren die Winterungen als Erstfrüchte im zweiten Jahr zwar schwächer als im ersten Jahr, der Mais konnte jedoch im zweiten Jahr kräftig zulegen und dies ausgleichen bzw. führte damit sogar zu einem höheren Gesamtertrag des Zweikulturnutzungssystems. Während im ersten Versuchsjahr Erst- und Zweitfrucht jeweils etwa die Hälfte zum Gesamtertrag beitrugen, war im zweiten Versuchsjahr der Mais mit einem Anteil von etwa zwei Drittel am Gesamtertrag deutlich stärker. Die Trockenmasseerträge der Erstfrüchte in den Varianten des Zweikulturnutzungssystems unterschieden sich kaum voneinander. Nur die Winterackerbohnen blieben etwas hinter den anderen Varianten zurück.

Im zweiten Versuchsjahr war bei den Erstfrüchten des Zweikulturnutzungssystems ein höheres Unkrautaufkommen zu finden als im ersten Versuchsjahr. Abbildung 6 zeigt stellvertretend für die Erstfrüchte das Gemenge aus Winterackerbohnen und Wintertriticale im ersten $(a, c)$ und zweiten Versuchsjahr (b, d). Hohes Auftreten zeigten neben anderen weiniger häufig vorkommenden Arten die Vogelmiere (Stellaria media), das Klettenlabkraut (Galium aparine), die Geruchlose Kamille (Matricaria inodora) und das Einjährige Rispengras (Poa annua).
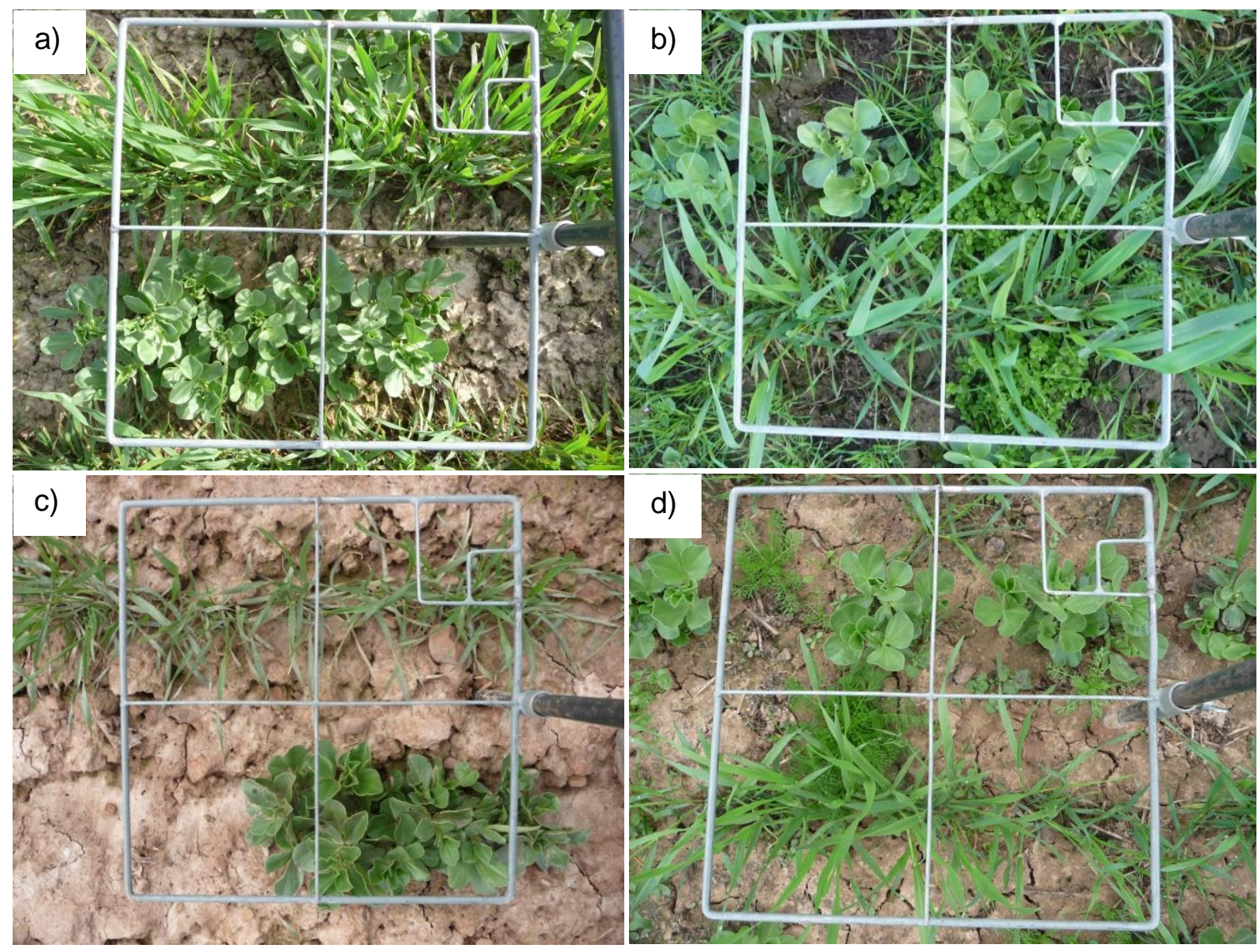

Abbildung 6: Pflanzenbestände des Gemenges aus Winterackerbohnen und Wintertriticale als Erstfrucht im Zweikulturnutzungssystem mit Mais a) am Standort Reinshof im Versuchsjahr 2015 (Aufnahmedatum 13. April 2015), b) am Standort Reinshof im Versuchsjahr 2016 (Aufnahmedatum 6. April 2016), c) am Standort Schoningen im Versuchsjahr 2015 (Aufnahmedatum 7. April 2015) und d) am Standort Schoningen im Versuchsjahr 2016 (Aufnahmedatum 13. April 2016). 


\section{Schoningen}

Deutlich unter dem Standort Reinshof blieben die Trockenmasseerträge am Standort Schoningen. Einzig das Deutsche Weidelgras übertraf hier im zweiten Versuchsjahr mit nahezu 200 dt TM ha-1 den Trockenmasseertrag am Standort Reinshof mit etwa 190 dt TM ha-1 (Abbildung 7).
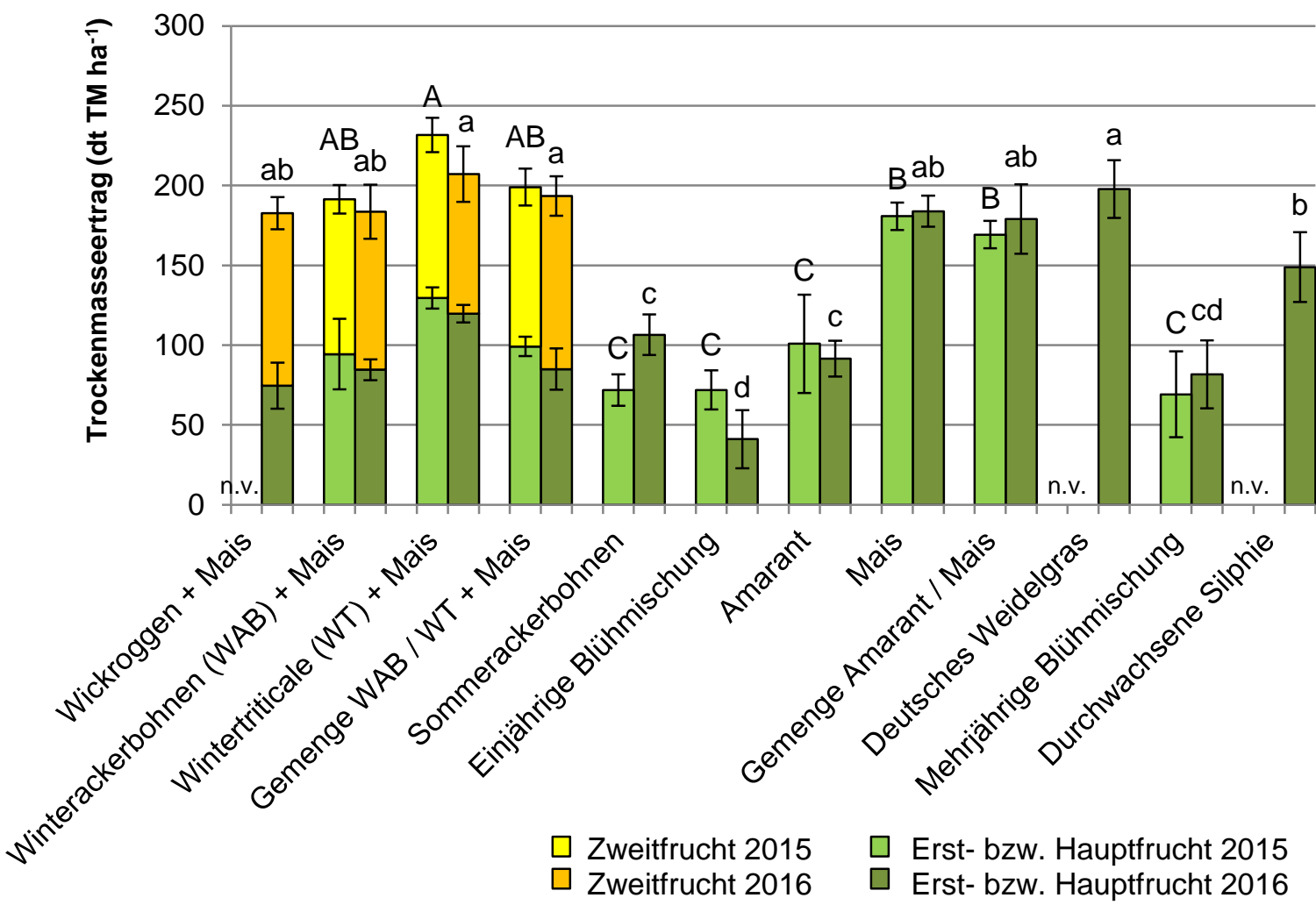

Abbildung 7: Trockenmasseerträge in den Versuchsjahren 2015 und 2016 am Standort Schoningen. Dargestellt sind die Mittelwerte \pm Standardabweichung. Varianten mit gleichen Großbuchstaben (2015) bzw. gleichen Kleinbuchstaben (2016) sind nicht signifikant verschieden, $p<0,05$ (Tukey-Test). Bei gestapelten Säulen beziehen sich die Angaben auf die Höhe der Gesamtsäule. n.v. = Werte nicht verfügbar.

Im ersten Versuchsjahr erzielten die Varianten des Zweikulturnutzungssystems die höchsten Trockenmasseerträge. Besonders die Variante aus Wintertriticale als Erst- und Mais als Zweitfrucht trat mit etwa $230 \mathrm{dt} \mathrm{TM} \mathrm{ha-1}$ hervor. Signifikant verschieden zu den anderen Varianten des Zweikulturnutzungssystems war diese Variante jedoch nicht, wohl aber zu Mais als Sommerhauptfrucht mit 180 dt TM ha-1 ${ }^{-1}$ Die schwächsten Varianten waren, wie am Standort Reinshof auch, die Sommerackerbohnen (72 dt TM ha-1), die ein- und mehrjährige Blühmischung (72 bzw. $69 \mathrm{dt} \mathrm{TM} \mathrm{ha-1)} \mathrm{sowie} \mathrm{der} \mathrm{Amarant} \mathrm{(101} \mathrm{dt} \mathrm{TM} \mathrm{ha-1).}$

Im zweiten Versuchsjahr wurde, wie im ersten Versuchsjahr auch, der höchste Trockenmasseertrag mit $208 \mathrm{dt} \mathrm{ha}^{-1}$ bei der Variante aus Wintertriticale als Erst- und Mais als Zweitfrucht gemessen. Den zweithöchsten Ertrag erzielte das Deutsche Weidelgras mit 198 dt TM ha-1 und war damit nicht signifikant verschieden dazu (Abbildung 7). Mit etwa $100 \mathrm{dt} \mathrm{TM} \mathrm{ha}{ }^{-1}$ trug der erste Schnitt des Deutschen Weidelgrases hier den größten Anteil zum Gesamt-Trockenmasseertrag bei, gefolgt vom zweiten Schnitt mit 60 und dem dritten Schnitt mit 40 dt TM ha-1 (Daten nicht dargestellt). Ähnlich gut schnitten auch der Mais, das Gemenge aus Amarant und 
Mais sowie die übrigen Varianten des Zweikulturnutzungssystems mit Trockenmasseerträgen im Bereich von 180 bis $190 \mathrm{dt} \mathrm{ha}^{-1}$ ab (Abbildung 7). Die Durchwachsene Silphie war zwar mit 150 dt TM ha ${ }^{-1}$ etwas schwächer, unterschied sich aber dennoch nicht signifikant von Mais, dem Gemenge aus Amarant und Mais sowie den Varianten des Zweikulturnutzungssystems Wickroggen und Winterackerbohnen, jeweils gefolgt von Mais. Mit $41 \mathrm{dt}$ TM ha-1 war die einjährigen Blühmischung die schwächste Variante im Vergleich mit den anderen Varianten aber auch im Vergleich der Versuchsjahre und Standorte. Die mehrjährige Blühmischung erzielte mit 82 dt TM ha-1 zwar genau den doppelten Ertrag, aufgrund der großen Streuung in den Erträgen der Blühmischungen, unterschied sie sich damit jedoch nicht signifikant von der einjährigen Blühmischung. Die Sommerackerbohnen und der Amarant blieben wie im ersten Jahr auch, weit hinter den ertragreichen Varianten zurück.

Beim Gemenge aus Amarant und Mais war die Zusammensetzung der einzelnen Komponenten am Gesamtertrag mit 8 bzw. $3 \%$ im ersten und zweiten Versuchsjahr ähnlich dem Standort Reinshof (Daten nicht dargestellt).

Bei den Varianten des Zweikulturnutzungssystems trugen am Standort Schoningen in beiden Versuchsjahren Erst- und Zweitfrucht nahezu mit gleichen Anteilen zum Gesamtertrag bei (Abbildung 7). Besonders ertragreich innerhalb der Erstfrüchte war die Wintertriticale mit 130 (1. Versuchsjahr) bzw. 120 dt TM ha-1 (2. Versuchsjahr) und damit auch deutlich ertragsstärker als einige Sommerhauptfrüchte.

Vergleichsweise große, jedoch nicht signifikante Unterschiede zwischen den Versuchsjahren gab es am Standort Schoningen bei den Sommerackerbohnen und der einjährigen Blühmischung. Während die Sommerackerbohnen die Erträge vom ersten zum zweiten Versuchsjahr um $47 \%$ steigern konnten, nahm der Ertrag bei der einjährigen Blühmischung deutlich um $57 \%$ ab.

Am Standort Schoningen wurden im zweiten Versuchsjahr bei der einjährigen Blühmischung zusätzlich zu den in der Mischung enthaltenen Blühpflanzen etwa 12 dt TM ha-1 an Unkrautbiomasse erfasst (Daten nicht dargestellt). Das hohe Aufkommen von vor allem der Geruchlosen Kamille wird in Abbildung $8 f$ erkennbar. Bei der mehrjährigen Blühmischung am Standort Schoningen war es eine Unkrautbiomasse von etwa $10 \mathrm{dt} \mathrm{TM} \mathrm{ha-1} \mathrm{im} \mathrm{ersten} \mathrm{und} 7 \mathrm{dt} \mathrm{TM} \mathrm{ha}^{-1}$ im zweiten Versuchsjahr (Daten nicht dargestellt). Abbildung $8 \mathrm{~g}$ zeigt die mehrjährige Blühmischung hier mit einem Unterwuchs an Vogelmiere, Abbildung 8h mit Besatz der Geruchlosen Kamille im zweiten Versuchsjahr. Am Standort Reinshof dagegen traten bei beiden Blühmischungen und in beiden Versuchsjahren kaum Unkräuter auf (Abbildung 8a, b, c und d).

Am Standort Reinshof ging der Bestand der Durchwachsenen Silphie nach einem Gewitter am 28. August 2016 teilweise ins Lager (Abbildung 9a). Am Standort Schoningen zeigte diese Variante bei der Bonitur am 18. Juli 2016 Welkeerscheinungen in der Form von abgestorbenen Blättern am gesamten Stängel (Abbildung 9b). Am Standort Reinshof war hingegen keine Welke erkennbar. 

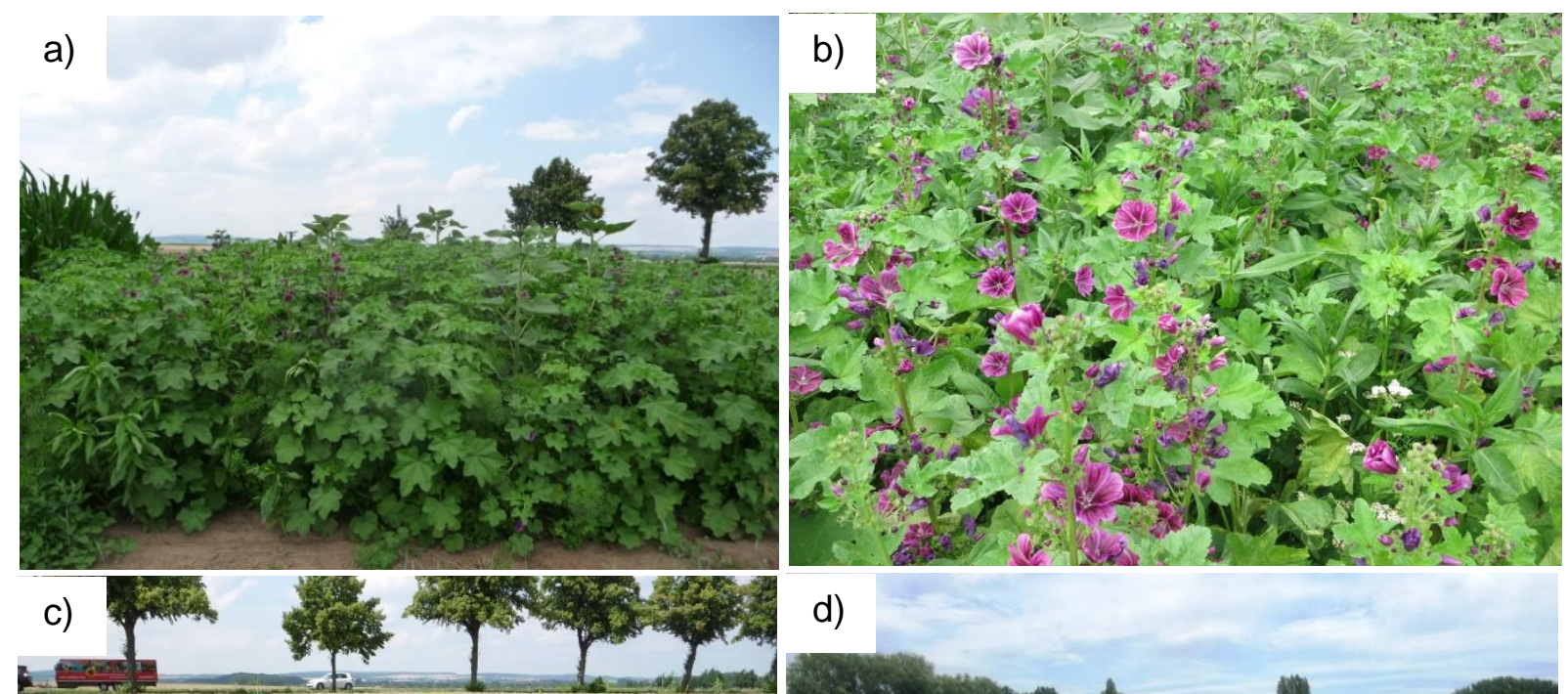

d)
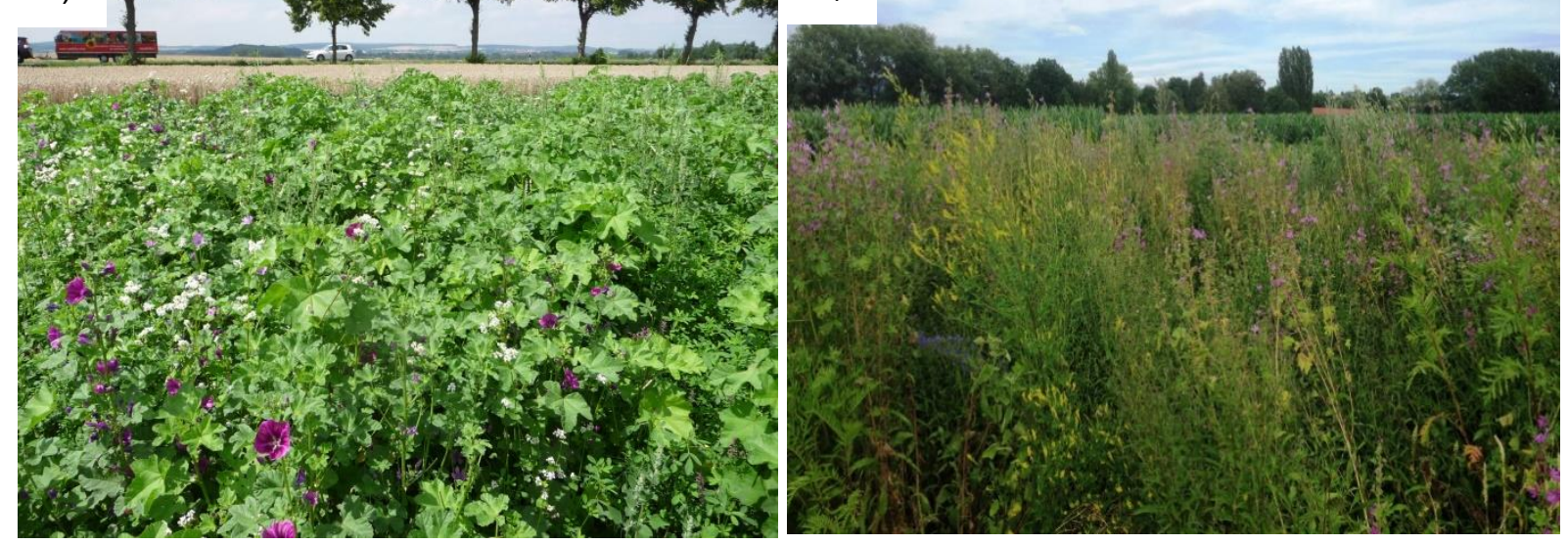

e)
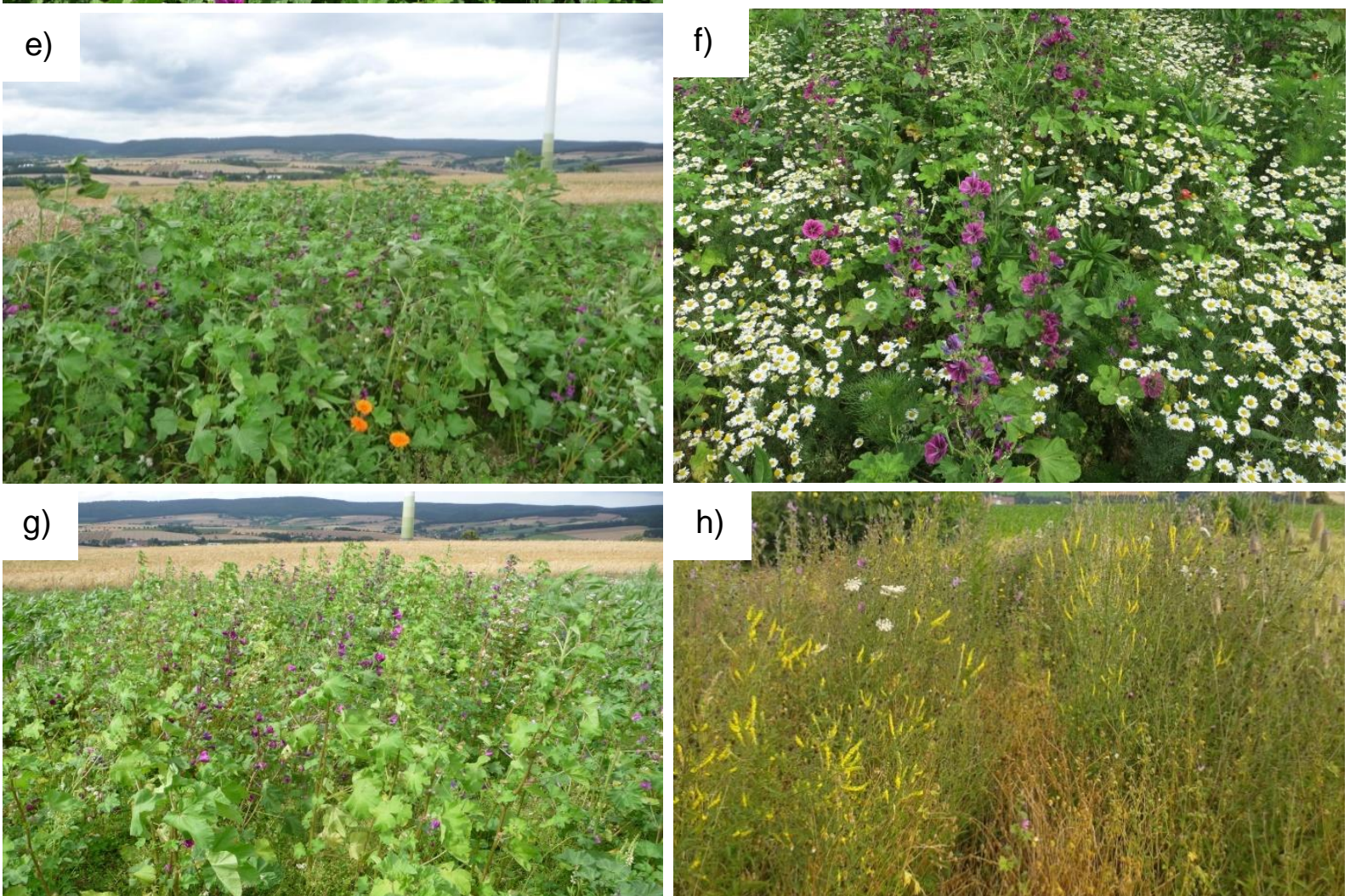

Abbildung 8: Pflanzenbestände der einjährigen Blühmischung a) am Standort Reinshof im Versuchsjahr 2015 am 23. Juli 2015 und b) am Standort Reinshof im Versuchsjahr 2016 am 21. Juli 2016. Pflanzenbestände der mehrjährigen Blühmischung c) am Standort Reinshof im Versuchsjahr 2015 am 23. Juli 2015 und d) am Standort Reinshof im Versuchsjahr 2016 am 10. Juli 2016. Pflanzenbestände der einjährigen Blühmischung e) am Standort Schoningen im Versuchsjahr 2015 am 28. Juli 2015 und f) am Standort Schoningen im Versuchsjahr 2016 am 18. Juli 2016. Pflanzenbestände der mehrjährigen Blühmischung g) am Standort Schoningen im Versuchsjahr 2015 am 28. Juli 2015 und h) am Standort Schoningen im Versuchsjahr 2016 am 18. Juli 2016. 

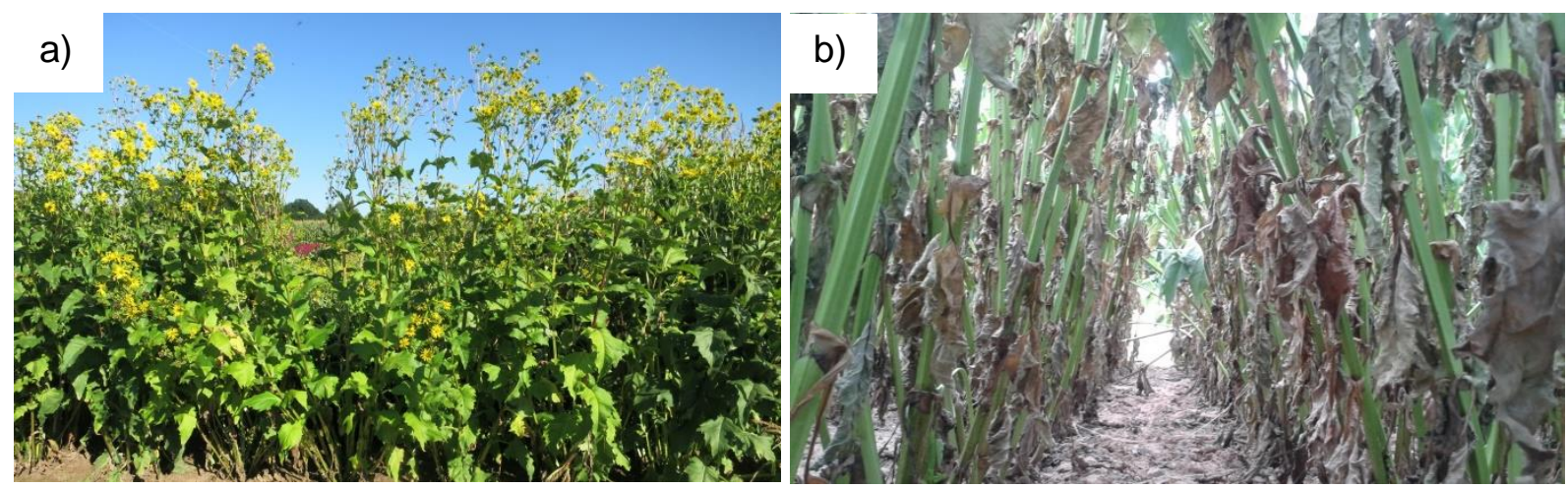

Abbildung 9: Pflanzenbestände der Durchwachsenen Silphie a) am Standort Reinshof im Versuchsjahr 2016 am 30. August 2016 und b) am Standort Schoningen im Versuchsjahr 2016 am 18. Juli 2016.

\subsubsection{Trockensubstanzgehalt}

\section{Reinshof}

Der Gehalt an Trockensubstanz (TS) in der Frischmasse bei der Ernte unterschied sich in beiden Versuchsjahren z.T. stark zwischen den Varianten (Tabelle 7). Silierfähig gilt die Biomasse bei einem TS-Gehalt zwischen 28 und $35 \%$. TS-Gehalte im silierfähigen Bereich wurden von dem Gemenge aus Amarant und Mais (35 bzw. $33 \%$ ) in beiden Versuchsjahren sowie von Mais (34\%), der mehrjährigen Blühmischung (32\%) und dem Wickroggen (28\%) im zweiten Versuchsjahr erreicht. Etwas oberhalb des angestrebten TS-Bereichs lag der Mais im ersten Versuchsjahr mit etwa $37 \%$. Die geringsten TS-Gehalte mit etwa $13 \%$ zeigten die Winterackerbohnen in beiden Versuchsjahren. Bei den Erstfrüchten im Zweikulturnutzungssystem erreichten die getreidebetonten Varianten die höchsten TS-Gehalte (24-28 \% bei Wintertriticale und Wickroggen). Wurden die Winterackerbohnen im Gemenge mit Wintertriticale angebaut, wurde immerhin ein TS-Gehalt von $21 \%$ im ersten und $22 \%$ im zweiten Versuchsjahr erreicht. Die Zweitfrucht Mais im Zweikulturnutzungssystem konnte zumindest im zweiten Versuchsjahr mit 33,3\% einen TS-Gehalt im optimalen Bereich erzielen; im ersten Versuchsjahr dagegen war der TS-Gehalt mit 21,6\% sehr gering (Daten nicht dargestellt).

Auch die Sommerackerbohnen, die einjährige Blühmischung und der Amarant blieben mit etwa 17 bis $24 \%$ TS in beiden Versuchsjahren weit vom angestrebten TS-Gehalt entfernt. Der TS-Gehalt der mehrjährigen Blühmischung im ersten Versuchsjahr war ähnlich niedrig wie der der einjährigen Blühmischung und unterschied sich damit deutlich vom zweiten Versuchsjahr. Beim Deutschen Weidelgras waren die TS-Gehalte der gemittelten Schnitte mit 23 bzw. $26 \%$ zumindest im ersten Versuchsjahr vergleichsweise gering (Tabelle 7). Dabei hatte das Deutsche Weidelgras beim ersten (Juni 2015 und 2016) und dritten Schnitt (Oktober 2015 und November 2016) TS-Gehalte von etwa $20 \%$, beim zweiten Schnitt im August des ersten Versuchsjahres $27 \%$ und im August des zweiten Versuchsjahres $35 \%$ (Daten nicht dargestellt). 
Tabelle 7: Trockensubstanzgehalte in den Versuchsjahren 2015 und 2016 am Standort Reinshof.

\begin{tabular}{|c|c|c|c|}
\hline Variante & $\begin{array}{c}2015 \\
(\%)\end{array}$ & $\begin{array}{c}2016 \\
(\%)\end{array}$ & \\
\hline Wickroggen & $25,7 \pm 1,1 \quad b$ & $28,4 \pm 0,9 \quad b$ & * \\
\hline Winterackerbohnen (WAB) & $13,9 \pm 0,9 f$ & $13,3 \pm 0,5 \mathrm{f}$ & \\
\hline Wintertriticale (WT) & $23,9 \pm 0,9 b c$ & $26,9 \pm 0,9 b$ & * \\
\hline Gemenge WAB / WT & $20,9 \pm 1,5 \mathrm{~cd}$ & $22,0 \pm 1,0 \mathrm{~d}$ & \\
\hline Sommerackerbohnen & $16,7 \pm 0,7$ ef & $18,1 \pm 1,2 \mathrm{e}$ & \\
\hline Einjährige Blühmischung & $23,6 \pm 3,4 b c$ & $21,2 \pm 2,3 \mathrm{de}$ & * \\
\hline Amarant & $19,8 \pm 0,8 \mathrm{de}$ & $22,8 \pm 0,8 \mathrm{~cd}$ & * \\
\hline Mais & $36,7 \pm 1,3$ a & $34,4 \pm 1,5$ a & * \\
\hline Gemenge Amarant / Mais & $35,3 \pm 0,6 a$ & $32,9 \pm 0,9 a$ & * \\
\hline Deutsches Weidelgras ${ }^{1)}$ & $22,7 \pm 3,9 \mathrm{bcd}$ & $25,5 \pm 7,5 \mathrm{bc}$ & * \\
\hline Mehrjährige Blühmischung & $24,5 \pm 1,7 \quad b$ & $31,8 \pm 1,4 a$ & * \\
\hline Durchwachsene Silphie & n.v. & $21,5 \pm 1,1 d$ & \\
\hline
\end{tabular}

Dargestellt sind die Mittelwerte \pm Standardabweichung. Varianten mit gleichen Buchstaben innerhalb eines Jahres sind nicht signifikant verschieden, $\mathrm{p}<0,05$ (Tukey-Test), * kennzeichnen signifikante Unterschiede zwischen den Versuchsjahren innerhalb einer Variante, $p<0,05$ (t-Test). n.v. = Wert nicht verfügbar.

1) Dargestellt ist der Mittelwert aus drei Schnitten.

\section{Schoningen}

Die TS-Gehalte der Biomasse zur Ernte der Varianten am Standort Schoningen waren überwiegend ähnlich denen am Standort Reinshof (Tabelle 8). Auffällig waren jedoch die hohen TS-Gehalte der Varianten Mais, dem Gemenge aus Amarant und Mais sowie der mehrjährigen Blühmischung im zweiten Versuchsjahr, die sich mit 40 bis $42 \%$ oberhalb des optimalen Bereichs für silierfähige Biomasse befanden.

TS-Gehalte im optimalen Bereich konnten von Mais (33\% TS) und dem Gemenge aus Amarant und Mais (32\% TS) im ersten Versuchsjahr aber auch von der Durchwachsenen Silphie (35\% TS) erreicht werden. Besonders gering waren die TS-Gehalte bei den Winterackerbohnen mit 16 bzw. $13 \%$ in beiden Versuchsjahren (Tabelle 8). Der Mais als Zweitfrucht im Zweikulturnutzungssystem zeigte wie am Standort Reinshof auch, im ersten Versuchsjahr mit 22,2 \% einen sehr geringen und im zweiten Versuchsjahr mit 29,7\% einen optimalen Trockensubstanzgehalt (Daten nicht dargestellt).

Das Deutsche Weidelgras erreichte hier im Mittel über die drei Schnitte des zweiten Versuchsjahres einem TS-Gehalt von $26 \%$ (Tabelle 8). Beim ersten (Juni 2016) und dritten Schnitt (Oktober 2016) waren TS-Gehalte von etwa $20 \%$ vorzufinden, beim zweiten Schnitt im August etwa $38 \%$ (Daten nicht dargestellt). 
Tabelle 8: Trockensubstanzgehalte in den Versuchsjahren 2015 und 2016 am Standort Schoningen.

\begin{tabular}{|c|c|c|c|}
\hline Variante & $\begin{array}{c}2015 \\
(\%)\end{array}$ & $\begin{array}{c}2016 \\
(\%)\end{array}$ & \\
\hline Wickroggen & n.v. & $24,9 \pm 1,8 \mathrm{c}$ & \\
\hline Winterackerbohnen (WAB) & $15,6 \pm 1,1 \mathrm{f}$ & $12,9 \pm 0,1 \mathrm{e}$ & \\
\hline Wintertriticale (WT) & $28,0 \pm 3,0$ bc & $25,0 \pm 1,6 \mathrm{c}$ & * \\
\hline Gemenge WAB / WT & $20,8 \pm 2,2$ de & $19,3 \pm 1,2 \mathrm{~d}$ & \\
\hline Sommerackerbohnen & $16,5 \pm 0,2$ ef & $18,8 \pm 0,3 \mathrm{~d}$ & \\
\hline Einjährige Blühmischung & $18,9 \pm 2,9$ def & $25,8 \pm 1,3 \mathrm{c}$ & * \\
\hline Amarant & $23,5 \pm 0,6 \mathrm{~cd}$ & $24,9 \pm 2,1 \quad c$ & \\
\hline Mais & $32,9 \pm 1,1$ a & $40,4 \pm 1,2 \quad a$ & * \\
\hline Gemenge Amarant / Mais & $31,5 \pm 1,2 a b$ & $41,4 \pm 3,5$ a & * \\
\hline Deutsches Weidelgras ${ }^{1)}$ & n.v. & $26,3 \pm 9,0 \quad c$ & \\
\hline $\begin{array}{l}\text { Mehrjährige Blühmischung } \\
\text { Durchwachsene Silphie }\end{array}$ & $\begin{array}{l}22,0 \pm 2,3 d \\
\text { n.v. }\end{array}$ & $\begin{array}{ll}42,3 \pm 3,0 & a \\
35,4 \pm 4,5 & b\end{array}$ & * \\
\hline
\end{tabular}

Dargestellt sind die Mittelwerte \pm Standardabweichung. Varianten mit gleichen Buchstaben innerhalb eines Jahres sind nicht signifikant verschieden, $p<0,05$ (Tukey-Test), * kennzeichnen signifikante Unterschiede zwischen den Versuchsjahren innerhalb einer Variante, $p<0,05$ (t-Test). n.v. = Werte nicht verfügbar.

1) Dargestellt ist der Mittelwert aus drei Schnitten.

\subsubsection{Stickstoff in der Spross-Trockenmasse}

\section{Reinshof}

Die untersuchten Varianten speicherten z.T. sehr große Mengen Stickstoff in ihrer oberirdischen Biomasse, jedoch unterschieden sich die aufgenommen N-Mengen zwischen den Varianten stark. Die mehrjährige Blühmischung speicherte im zweiten Versuchsjahr nur $129 \mathrm{~kg} \mathrm{~N}$ ha $^{-1}$, während die Variante Winterackerbohnen und Mais des Zweikulturnutzungssystems (Summe aus Erst- und Zweitfrucht) im zweiten Versuchsjahr $457 \mathrm{~kg} \mathrm{~N} \mathrm{ha}^{-1}$ aufnahm (Abbildung 10).

Die höchsten N-Aufnahmen zeigten die Varianten des Zweikulturnutzungssystems und dabei insbesondere die Winterackerbohnen mit nachfolgendem Mais, die neben dem zweiten auch im ersten Versuchsjahr mit $421 \mathrm{~kg} \mathrm{~N}^{-1} \mathrm{~g}^{-1}$ große Mengen Stickstoff aufnahmen. Etwas geringer, aber nicht signifikant verschieden, waren mit $360 \mathrm{~kg} \mathrm{~N} \mathrm{a}^{-1} \mathrm{im}$ ersten Jahr und $394 \mathrm{~kg} \mathrm{~N} \mathrm{ha}^{-1}$ im zweiten Jahr die N-Aufnahmen des Gemenges aus Winterackerbohnen und Wintertriticale als Erst- und Mais als Zweitfrucht. Bei diesen Varianten wurden durch die Erstfrüchte mit einem Anteil von etwa zwei Drittel im ersten und etwa der Hälfte im zweiten Versuchsjahr beachtliche $\mathrm{N}$-Mengen zur Gesamt-N-Akkumulation beigesteuert.

Auch die Sommerackerbohnen nahmen mit etwa $285 \mathrm{~kg} \mathrm{~N} \mathrm{ha}^{-1}$ in beiden Versuchsjahren große N-Mengen auf und unterschieden sich damit nicht signifikant von einigen Varianten des Zweikulturnutzungssystems. In einem ähnlichen Bereich bewegten sich auch der Mais und das Gemenge aus Amarant und Mais sowie, wenn auch mit geringeren, aber z.T. nicht signifikant geringeren N-Aufnahmen der Amarant, das Deutsche Weidelgras und die Durchwachsene Silphie. Die geringsten Stickstoffmengen wurden bei der ein- und mehrjährigen Blühmischung gemessen. 


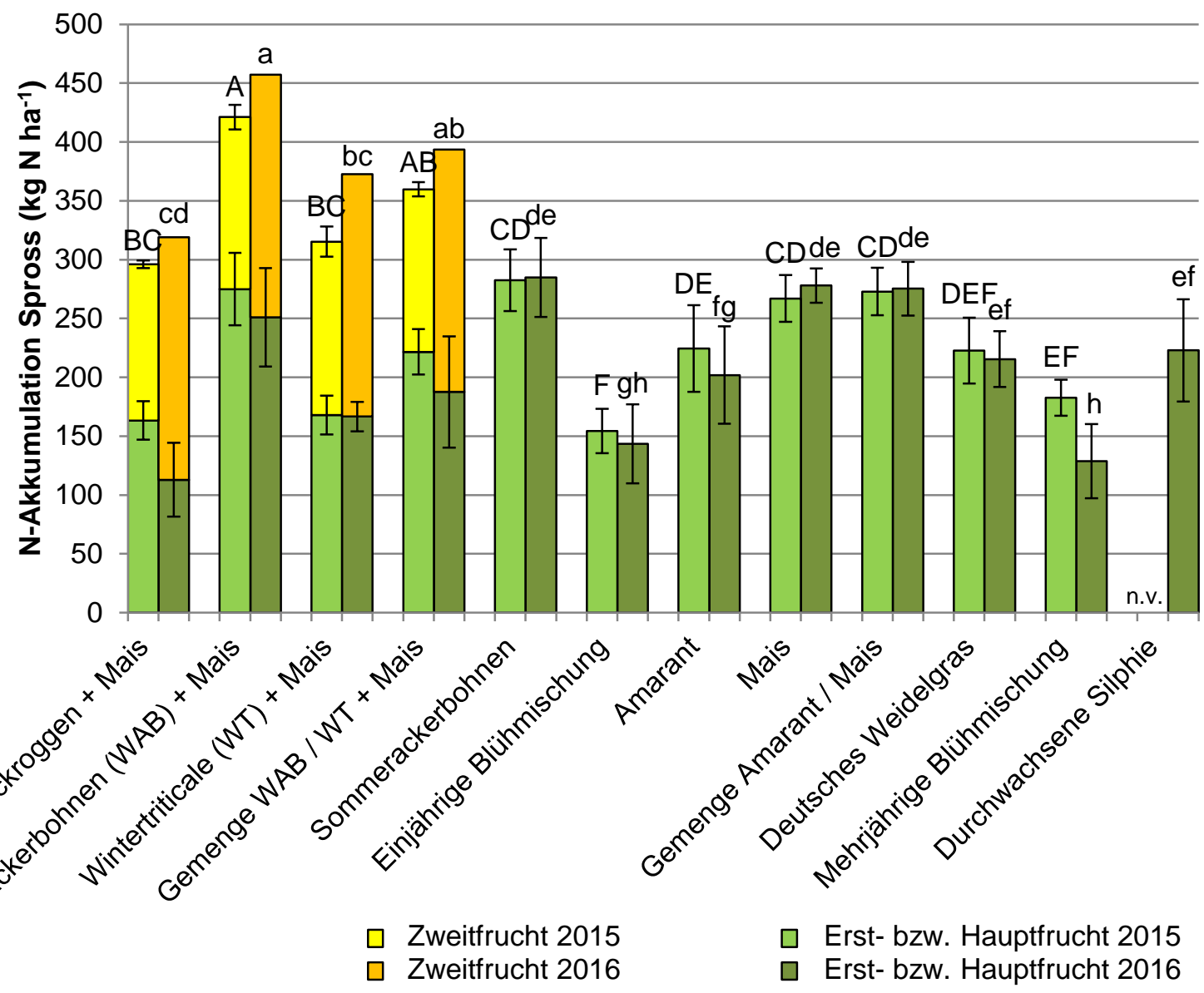

Abbildung 10: Stickstoffakkumulation in der oberirdischen Biomasse in den Versuchsjahren 2015 und 2016 am Standort Reinshof. Dargestellt sind die Mittelwerte \pm Standardabweichung. Varianten mit gleichen Großbuchstaben bzw. gleichen Kleinbuchstaben sind nicht signifikant verschieden, $p<0,05$ (Tukey-Test). Bei gestapelten Säulen beziehen sich die Angaben auf die Höhe der Gesamtsäule. n.v. = Wert nicht verfügbar.

Bei der mehrjährigen Blühmischung unterschieden sich die in der Biomasse akkumulierten Stickstoffmengen zwischen den Versuchsjahren mit $183 \mathrm{~kg} \mathrm{~N} \mathrm{ha}^{-1}$ im ersten und $129 \mathrm{~kg} \mathrm{~N}$ $\mathrm{ha}^{-1} \mathrm{im}$ zweiten Versuchsjahr. Signifikant war der Unterschied jedoch nicht. Hier wurde, wie auch bei den anderen Varianten des Zweikulturnutzungssystems, im zweiten Versuchsjahr mehr Stickstoff durch den Mais als Zweitfrucht zur Gesamtmenge beigesteuert als im ersten Versuchsjahr, zusätzlich aber auch von der Wintertriticale die gleiche N-Menge aufgenommen wie im ersten Versuchsjahr. Bei den anderen Varianten des Zweikulturnutzungssystems nahmen die Erstfrüchte hingegen im zweiten Versuchsjahr weniger Stickstoff auf als im ersten.

\section{Schoningen}

Auch am Standort Schoningen akkumulierten die Feldfrüchte große Mengen Stickstoff in der oberirdischen Biomasse. Mit N-Mengen zwischen $48 \mathrm{~kg} \mathrm{~N} \mathrm{ha}^{-1}$ bei der einjährigen Blühmischung im zweiten Versuchsjahr und $394 \mathrm{~kg} \mathrm{~N}^{-1}$ bei Winterackerbohnen und nachfolgendem Mais ebenfalls im zweiten Versuchsjahr gab es auch hier große Unterschiede zwischen den Varianten (Abbildung 11). 


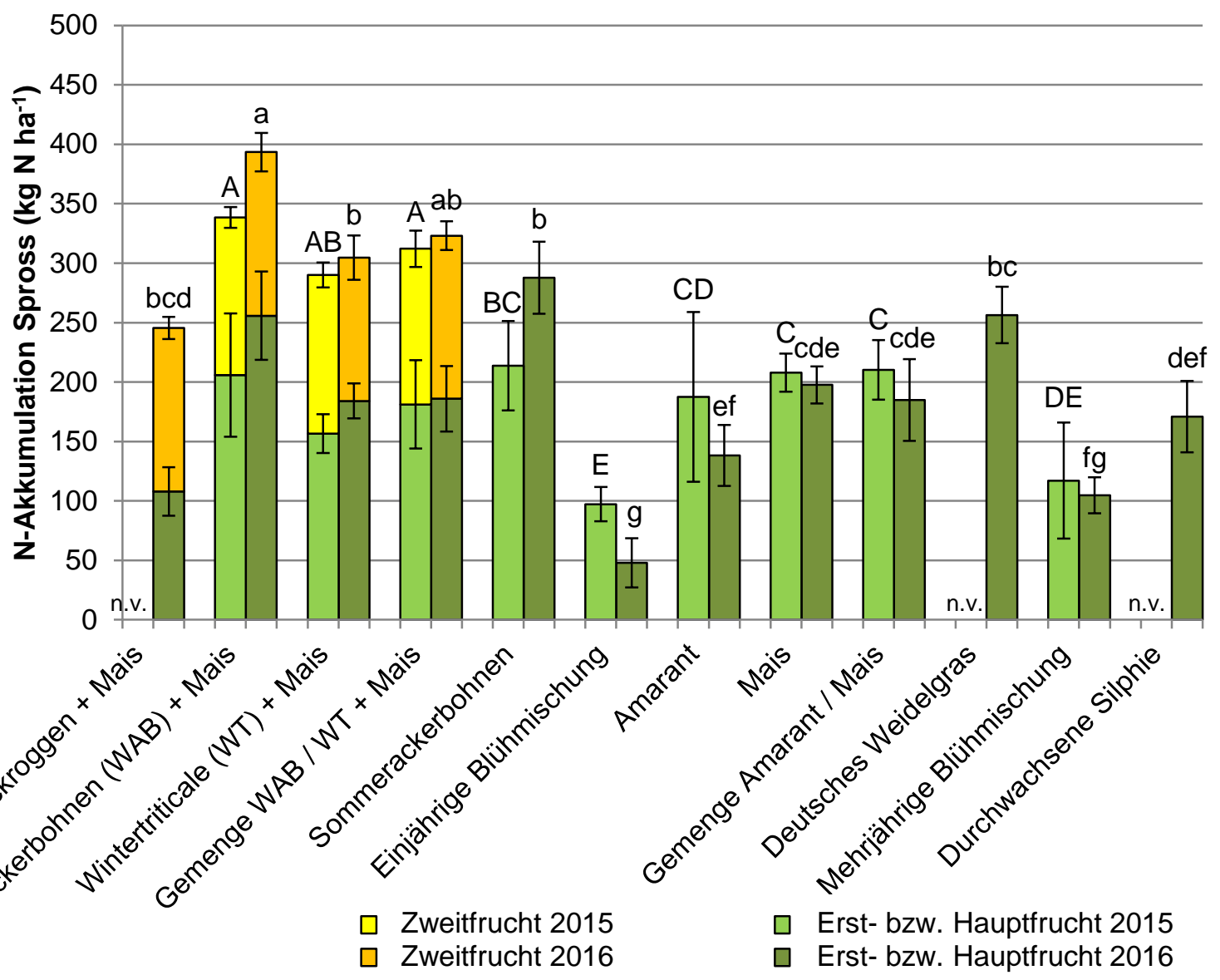

Abbildung 11: Stickstoffakkumulation in der oberirdischen Biomasse in den Versuchsjahren 2015 und 2016 am Standort Schoningen. Dargestellt sind die Mittelwerte \pm Standardabweichung. Varianten mit gleichen Großbuchstaben bzw. gleichen Kleinbuchstaben sind nicht signifikant verschieden, $p<0,05$ (Tukey-Test). Bei gestapelten Säulen beziehen sich die Angaben auf die Höhe der Gesamtsäule. n.v. = Werte nicht verfügbar.

Die Varianten des Zweikulturnutzungsystems akkumulierten in beiden Versuchsjahren die höchsten Stickstoffmengen. Im ersten Versuchsjahr waren die Varianten Winterackerbohnen, Wintertriticale und das Gemenge aus Winterackerbohnen und Wintertriticale jeweils gefolgt

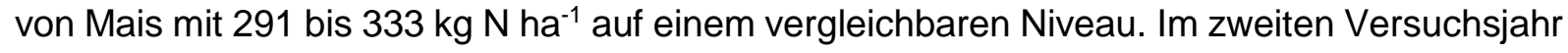
waren die Winterackerbohnen mit nachfolgendem Mais immerhin von den Varianten Wickroggen plus Mais und Wintertriticale plus Mais signifikant verschieden. Bis auf den Wickroggen trugen beim Zweikulturnutzungssystem die Erstfrüchte in beiden Versuchsjahren zu deutlich mehr als der Hälfte zur Gesamt-N-Akkumulation bei. Sehr hohe N-Mengen waren auch in der Biomasse der Sommerackerbohnen zu finden. Mit $288 \mathrm{~kg} \mathrm{~N}^{\mathrm{N}}{ }^{-1}$ im zweiten Versuchsjahr zogen sie mit einigen Varianten des Zweikulturnutzungssystems gleich und übertrafen sogar den Mais als Sommerhauptfrucht mit etwa $200 \mathrm{~kg} \mathrm{~N} \mathrm{ha}^{-1}$. Auf gleichem Niveau wie die Sommerackerbohnen war auch das Deutsche Weidelgras mit $256 \mathrm{~kg} \mathrm{~N} \mathrm{ha}^{-1}$.

Die geringsten N-Mengen in der oberirdischen Biomasse zeigten die ein- und mehrjährige Blühmischung. Auffällig bei der einjährigen Blühmischung war zudem die Halbierung der aufgenommenen N-Menge vom ersten zum zweiten Jahr. Ein ähnliches Bild zeigte sich auch beim Amarant. Signifikant waren diese Unterschiede jedoch nicht. Unterschiede zwischen den Versuchsjahren, jedoch ebenfalls nicht signifikant, zeigten auch die Winterackerbohnen im Zweikulturnutzungssystem mit Mais sowie die Sommerackerbohnen, wobei bei diesen Varianten das zweite Versuchsjahr zu höheren Mengen führte als das erste. 


\subsubsection{Spezifischer Methanertrag und Methanflächenertrag}

Die höchsten spezifischen Methanerträge konnten mit etwa $0,350 \mathrm{Nm}^{3} \mathrm{CH}_{4} \mathrm{~kg}^{-1}$ oTM bei Mais als Sommerhauptfrucht und dem Gemenge aus Amarant und Mais gemessen werden (Tabelle 9). Auch der Mais als Zweitfrucht blieb mit $0,341 \mathrm{Nm}^{3} \mathrm{CH}_{4} \mathrm{~kg}^{-1} \mathrm{oTM}$ nicht weit davon entfernt. Es folgten die drei Schnitte des Deutschen Weidelgrases (0,324 bis $0,331 \mathrm{Nm}^{3} \mathrm{CH}_{4} \mathrm{~kg}^{-1}$ oTM), die sich untereinander nicht wesentlich unterschieden und die getreidedominierenden Varianten als Erstfrüchte im Zweikulturnutzungssystems Wintertriticale $\left(0,331 \mathrm{Nm}^{3} \mathrm{CH}_{4} \mathrm{~kg}^{-1}\right.$ oTM) und Wickroggen $\left(0,327 \mathrm{Nm}^{3} \mathrm{CH}_{4} \mathrm{~kg}^{-1}\right.$ oTM). Deutlich geringer waren die Methanausbeuten bei den Winterackerbohnen, der ein- und mehrjährige Blühmischung sowie der Durchwachsenen Silphie mit Werten zwischen 0,284 und 0,266 $\mathrm{Nm}^{3} \mathrm{CH}_{4} \mathrm{~kg}^{-1}$ oTM. Auffällig waren die Methanausbeuten bei der mehrjährigen Blühmischung, die sich zwischen den Nutzungsjahren unterschieden, wobei die Biomasse im zweiten Nutzungsjahr weniger Methan lieferte als im ersten.

Tabelle 9: Spezifischer Methanertrag der untersuchten Varianten am Standort Reinshof im zweiten Versuchsjahr bzw. bei der mehrjährigen Blühmischung zusätzlich im ersten Versuchsjahr. Analysemethode: „Hohenheimer Biogasertragstest" nach VDI 4630.

\begin{tabular}{lc}
\hline Variante & $\begin{array}{c}\text { Spezifischer Methanertrag } \\
\left(\mathrm{Nm}^{3} \mathrm{CH}_{4} \mathrm{~kg}^{-1} \text { oTM }\right)\end{array}$ \\
\hline Mais & 0,356 \\
Gemenge Amarant / Mais & 0,354 \\
Mais Zweitfrucht & 0,341 \\
Deutsches Weidelgras 2. Schnitt & 0,332 \\
Wintertriticale (WT) & 0,331 \\
Deutsches Weidelgras 1. Schnitt & 0,331 \\
Wickroggen & 0,327 \\
Deutsches Weidelgras 3. Schnitt & 0,324 \\
Amarant & 0,315 \\
Gemenge WAB / WT & 0,312 \\
Sommerackerbohnen & 0,299 \\
Mehrjährige Blühmischung 1. Jahr & 0,292 \\
Winterackerbohnen (WAB) & 0,284 \\
Mehrjährige Blühmischung 2. Jahr & 0,271 \\
Einjährige Blühmischung & 0,270 \\
Durchwachsene Silphie & 0,266 \\
\hline
\end{tabular}

Die Methanflächenerträge ergeben sich aus den spezifischen Methanerträgen in $\mathrm{Nm}^{3} \mathrm{CH}_{4}$ $\mathrm{kg} \mathrm{oTM}^{-1}$ und den Erträgen der organischen Trockenmasse in $\mathrm{kg}^{\circ}$ TM ha-1. Am Standort Reinshof ergaben sich die höchsten Methanflächenerträge bei den Varianten, bei denen Mais beteiligt war (Tabelle 10). Im ersten Versuchsjahr lagen die Methanflächenerträge bei den Varianten des Zweikulturnutzungssystems zwischen 6330 (Winterackerbohnen + Mais) und 7719 $\mathrm{Nm}^{3} \mathrm{CH}_{4}$ ha-1 (Wickroggen + Mais). Sie unterschieden sich damit nicht signifikant von Mais als Sommerhauptfrucht mit $7376 \mathrm{Nm}^{3} \mathrm{CH}_{4}$ ha $^{-1}$. Im zweiten Versuchsjahr erbrachten die Varianten des Zweikulturnutzungssystems aus Wickroggen und Mais sowie Wintertriticale und Mais mit beachtlichen Werten von 9578 bzw. $9631 \mathrm{Nm}^{3} \mathrm{CH}_{4}$ ha' $^{-1}$ aber signifikant höhere Methanflächenerträge im Vergleich zu Mais als Sommerhauptfrucht mit $8108 \mathrm{Nm}^{3} \mathrm{CH}_{4} \mathrm{ha}^{-1}$. Deutlich geringere Methanflächenerträge ergaben sich für das Deutsche Weidelgras mit $6022 \mathrm{Nm}^{3} \mathrm{CH}_{4}$ 
ha ${ }^{-1}$ im ersten und $5622 \mathrm{Nm}^{3} \mathrm{CH}_{4}$ ha $^{-1}$ im zweiten Versuchsjahr und die Durchwachsene Silphie mit $5212 \mathrm{Nm}^{3} \mathrm{CH}_{4}$ ha $^{-1}$. Mit Methanflächenerträgen im Bereich von etwa 2500 bis $3400 \mathrm{Nm}^{3}$ $\mathrm{CH}_{4}$ ha $^{-1}$ waren die Sommerackerbohnen, die ein- und mehrjährige Blühmischung sowie der Amarant weit abgeschlagen.

Tabelle 10: Methanflächenerträge in den Versuchsjahren 2015 und 2016 an den Standorten Reinshof und Schoningen.

\begin{tabular}{lcccc}
\hline & \multicolumn{2}{c}{ Reinshof } & \multicolumn{2}{c}{ Schoningen } \\
Variante & $\begin{array}{l}2015 \\
\left(\mathrm{Nm}^{3} \mathrm{CH}_{4} \mathrm{ha}^{-1}\right)\end{array}$ & $\begin{array}{c}2016 \\
\left(\mathrm{Nm}^{3} \mathrm{CH}_{4} \mathrm{ha}^{-1}\right)\end{array}$ & $\begin{array}{c}2015 \\
\left(\mathrm{Nm}^{3} \mathrm{CH}_{4} \mathrm{ha}^{-1}\right)\end{array}$ & $\begin{array}{c}2016 \\
\left(\mathrm{Nm}^{3} \mathrm{CH}_{4} \mathrm{ha}^{-1}\right)\end{array}$ \\
\hline Wickroggen + Mais & $7719 \mathrm{a}$ & $9578 \mathrm{a} *$ & $\mathrm{n} . \mathrm{v}$. & $5740 \mathrm{a}$ \\
Winterackerbohnen + Mais & $6330 \mathrm{bc}$ & $8619 \mathrm{abc} *$ & $5589 \mathrm{~b}$ & $5402 \mathrm{a}$ \\
Wintertriticale + Mais & $7637 \mathrm{a}$ & $9631 \mathrm{a} *$ & $7281 \mathrm{a}$ & $6505 \mathrm{a}$ \\
Gemenge WAB / WT + Mais & $7149 \mathrm{abc}$ & $9086 \mathrm{ab} *$ & $6022 \mathrm{~b}$ & $5867 \mathrm{a}$ \\
Sommerackerbohnen & $2520 \mathrm{~d}$ & $3060 \mathrm{e}$ & $1965 \mathrm{C}$ & $2913 \mathrm{bc}$ \\
Einjährige Blühmischung & $3176 \mathrm{~d}$ & $3229 \mathrm{e}$ & $1719 \mathrm{C}$ & $982 \mathrm{~d}$ \\
Amarant & $3336 \mathrm{~d}$ & $3410 \mathrm{e}$ & $2739 \mathrm{c}$ & $2487 \mathrm{bc}$ \\
Mais & $7376 \mathrm{ab}$ & $8108 \mathrm{bc} *$ & $6165 \mathrm{ab}$ & $6271 \mathrm{a}$ \\
Gemenge Amarant / Mais & $7753 \mathrm{a}$ & $7788 \mathrm{c}$ & $5679 \mathrm{~b}$ & $6062 \mathrm{a}$ \\
Deutsches Weidelgras & $6022 \mathrm{c}$ & $5622 \mathrm{~d}$ & n.v. & $5886 \mathrm{a}$ \\
Mehrjährige Blühmischung & $2511 \mathrm{~d}$ & $3304 \mathrm{e} *$ & $1805 \mathrm{c}$ & $2052 \mathrm{~cd}$ \\
Durchwachsene Silphie & n.v. & $5212 \mathrm{~d}$ & n.v. & $3505 \mathrm{~b}$ \\
\hline
\end{tabular}

Dargestellt sind die Mittelwerte, Varianten mit gleichen Buchstaben an einem Standort innerhalb eines Jahres sind nicht signifikant verschieden, $p<0,05$ (Tukey-Test), * kennzeichnen signifikante Unterschiede zwischen den Versuchsjahren innerhalb einer Variante an einem Standort, $p<0,05$ (t-Test), n.v. = Werte nicht verfügbar.

Am Standort Schoningen zeigte sich im ersten Versuchsjahr ein ähnliches Bild in der Rangfolge der Methanflächenerträge der Varianten wie am Standort Reinshof, jedoch auf einem niedrigeren Niveau: Die Varianten des Zweikulturnutzungssystems unterschieden sich mit Methanflächenerträgen zwischen 5589 und $7281 \mathrm{Nm}^{3} \mathrm{CH}_{4}$ ha-1 nicht signifikant von Mais als Sommerhauptfrucht mit $6165 \mathrm{Nm}^{3} \mathrm{CH}_{4} \mathrm{ha}^{-1}$. Die übrigen Varianten blieben deutlich dahinter zurück.

Für das Deutsche Weidelgras ergab sich mit $5886 \mathrm{Nm}^{3} \mathrm{CH}_{4}$ ha-1 ein Methanflächenertrag, der bei den hochleistenden maisgeprägten Varianten (Zweikulturnutzungssystem, Mais und Gemenge aus Amarant und Mais) mit Werten von 5402 bis $6505 \mathrm{Nm}^{3} \mathrm{CH}_{4}$ ha-1 mithalten konnte. Den geringsten Methanflächenertrag mit $982 \mathrm{Nm}^{3} \mathrm{CH}_{4}$ ha-1 zeigte die einjährige Blühmischung im zweiten Versuchsjahr. Signifikant unterschiedlich waren die Versuchsjahre bei der einjährigen Blühmischung aber nicht. Anders als am Standort Reinshof nahmen hier auch die Methanflächenerträge bei den Varianten des Zweikulturnutzungssystems vom ersten zum zweiten Versuchsjahr ab. Am Standort Reinshof nahmen sie im gleichen Zeitraum signifikant zu.

\subsubsection{Spurenelementgehalte Cobalt und Nickel}

\section{Reinshof}

Am Standort Reinshof hatten in beiden Versuchsjahren die Sommer- $(0,208$ bzw. 0,167 mg $\left.\mathrm{kg}^{-1} \mathrm{TM}\right)$ und Winterackerbohnen (0,114 bzw. 0,141 $\left.\mathrm{mg} \mathrm{kg}^{-1} \mathrm{TM}\right)$ die höchsten Cobaltgehalte (Tabelle 11a). Keine der anderen Varianten konnte auch nur annähernd an diese Varianten 
herankommen. Mit 0,048 bis 0,062 $\mathrm{mg} \mathrm{Co} \mathrm{kg}^{-1} \mathrm{TM}$ waren Amarant, das Gemenge aus Winterackerbohnen und Wintertriticale sowie die mehrjährige Blühmischung die Varianten mit den zweithöchsten Cobaltgehalten. Die geringsten Cobaltgehalte waren mit Werten zwischen 0,006 und 0,021 $\mathrm{mg} \mathrm{kg}^{-1}$ TM bei Mais, dem Gemenge aus Amarant und Mais, den getreidebetonten Varianten der Erstfrüchte des Zweikulturnutzungssystems sowie dem Deutschen Weidelgras zu finden.

Die höchsten Nickelgehalte am Standort Reinshof zeigten, wie auch beim Cobalt, die Sommerackerbohnen mit 0,897 $\mathrm{mg} \mathrm{kg}^{-1} \mathrm{TM}$ im ersten und 0,814 $\mathrm{mg} \mathrm{kg}^{-1} \mathrm{TM}$ im zweiten Versuchsjahr (Tabelle 11a). Auch beim Deutschen Weidelgras wurden hohe Nickelgehalte gemessen. Mit 0,750 $\mathrm{mg} \mathrm{Ni} \mathrm{kg}^{-1}$ TM unterschied es sich im zweiten Versuchsjahr nicht signifikant von den Sommerackerbohnen. Etwas weniger Nickel nahmen die Winterackerbohnen (0,630 bzw. $0,621 \mathrm{mg} \mathrm{Ni} \mathrm{kg}^{-1} \mathrm{TM}$ ) auf, unterschieden sich aber nicht signifikant vom Deutschen Weidelgras. Wie auch bei den Cobaltgehalten, waren der Mais, das Gemenge aus Amarant und Mais sowie der Wickroggen und die Wintertriticale aber auch die einjährige Blühmischung die Varianten mit den geringsten Nickelgehalten.

\section{Schoningen}

Am Standort Schoningen waren, wie am Standort Reinshof auch, die Sommerackerbohnen mit 0,284 bzw. 0,254 $\mathrm{mg} \mathrm{kg}^{-1} \mathrm{TM}$ in beiden Versuchsjahren die Variante mit den höchsten Cobaltgehalten (Tabelle 11b). Anders als am Reinshof blieben die Winterackerbohnen (0,095 $\mathrm{mg} \mathrm{kg}^{-1} \mathrm{TM}$ ) im ersten Versuchsjahr hier deutlich dahinter zurück. Höhere, wenn auch nicht signifikant höhere Werte als die Winterackerbohnen zeigten sogar die einjährige Blühmischung und der Amarant mit 0,129 bzw. 0,109 $\mathrm{mg} \mathrm{Co} \mathrm{kg}^{-1} \mathrm{TM}$.

Im zweiten Versuchsjahr waren an diesem Standort mit den Sommerackerbohnen auch die Winterackerbohnen, die ein- und mehrjährige Blühmischung sowie der Amarant mit Cobaltgehalten zwischen 150 und $198 \mathrm{mg} \mathrm{kg}^{-1} \mathrm{TM}$ statistisch gleichauf. Mit 0,144 $\mathrm{mg} \mathrm{kg}^{-1} \mathrm{TM}$ war sogar das Gemenge aus Winterackerbohnen und Wintertriticale mit dieser Gruppe vergleichbar. Die geringsten Cobaltgehalte zeigten in beiden Versuchsjahren, wie am Reinshof auch, der Mais, das Gemenge aus Amarant und Mais sowie die Wintertriticale, ebenso die nur im zweiten Versuchsjahr geprüften Varianten Wickroggen und Deutsches Weidelgras.

Im ersten Versuchsjahr wurden am Standort Schoningen die mit Abstand höchsten Nickelgehalte mit 0,601 $\mathrm{mg} \mathrm{kg}^{-1} \mathrm{TM}$ von den Sommerackerbohnen erreicht (Tabelle 11b). Es folgte eine Gruppe aus Winterackerbohnen, Gemenge aus Winterackerbohnen und Wintertriticale, einund mehrjähriger Blühmischung sowie Amarant, die mit 0,122 bis 0,287 mg Ni kg-1 TM die zweithöchsten Nickelgehalte zeigten. Im zweiten Versuchsjahr akkumulierten die Sommerackerbohnen zwar weniger Nickel in der Biomasse als im ersten, wiesen aber dennoch mit $0,456 \mathrm{mg} \mathrm{Ni} \mathrm{kg}^{-1} \mathrm{TM}$ die höchsten Gehalte auf. Etwas geringer war die Nickelaufnahme beim

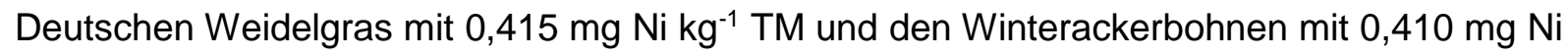
$\mathrm{kg}^{-1}$ TM. Statistisch signifikant war der Unterschied zu den Sommerackerbohnen damit jedoch nicht. Mittlere Nickelgehalte mit Werten von 0,261 bis $0,282 \mathrm{mg} \mathrm{kg}^{-1} \mathrm{TM}$ wiesen erneut das Gemenge aus Winterackerbohnen und Wintertriticale sowie die ein- und mehrjährige Blühmischung auf. Aber auch der Wickroggen und die Durchwachsene Silphie ordneten sich in diese Gruppe ein. Besonders gering waren wieder die Nickelgehalte von Mais, dem Gemenge aus Amarant und Mais sowie der Wintertriticale. 
Tabelle 11: Cobalt- und Nickelgehalte im oberirdischen Aufwuchs in den Versuchsjahren 2015 und 2016 an den Standorten Reinshof (a) und Schoningen (b).

\begin{tabular}{|c|c|c|c|c|c|c|c|}
\hline \multirow[t]{3}{*}{ a) } & \multicolumn{7}{|c|}{ Reinshof } \\
\hline & \multicolumn{5}{|c|}{ Cobalt (mg kg $\left.{ }^{-1} \mathrm{TM}\right)$} & \multicolumn{2}{|c|}{ Nickel (mg kg$\left.{ }^{-1} \mathrm{TM}\right)$} \\
\hline & 2015 & & 2016 & & & 2015 & 2016 \\
\hline Wickroggen & $0,015 \pm 0,008$ & $d$ & $0,007 \pm 0,002 \mathrm{e}$ & & & $0,178 \pm 0,039 \mathrm{e}$ & $0,108 \pm 0,043 \quad f$ \\
\hline Winterackerbohnen (WAB) & $0,114 \pm 0,016$ & $b$ & $0,141 \pm 0,035$ & a & & $0,630 \pm 0,106 b$ & $0,621 \pm 0,071 \quad b$ \\
\hline Wintertriticale (WT) & $0,011 \pm 0,002$ & $d$ & $0,009 \pm 0,004 \mathrm{de}$ & & & $0,224 \pm 0,115 \mathrm{de}$ & $0,115 \pm 0,097$ ef \\
\hline Gemenge WAB / WT & $0,050 \pm 0,007$ & $c$ & $0,057 \pm 0,007$ & & & $0,347 \pm 0,040 \mathrm{~cd}$ & $0,337 \pm 0,060 \quad \mathrm{c}$ \\
\hline Sommerackerbohnen & $0,208 \pm 0,024$ & a & $0,167 \pm 0,039$ & & & $0,897 \pm 0,069$ a & $0,814 \pm 0,071 \quad a$ \\
\hline Einjährige Blühmischung & $0,022 \pm 0,017$ & $d$ & $0,031 \pm 0,005$ & & & $0,187 \pm 0,053 \mathrm{e}$ & $0,249 \pm 0,022 \mathrm{cde}$ \\
\hline Amarant & $0,048 \pm 0,009$ & $c$ & $0,053 \pm 0,004 b$ & & & $0,154 \pm 0,041 \mathrm{e}$ & $0,191 \pm 0,061$ def \\
\hline Mais & $0,009 \pm 0,007$ & $d$ & $0,006 \pm 0,004$ & e & & $0,126 \pm 0,025 \mathrm{e}$ & $0,144 \pm 0,050$ ef \\
\hline Gemenge Amarant / Mais & $0,010 \pm 0,009$ & $d$ & $0,009 \pm 0,007 \mathrm{de}$ & & & $0,126 \pm 0,024 \mathrm{e}$ & $0,146 \pm 0,020$ ef \\
\hline Deutsches Weidelgras & $0,018 \pm 0,007$ & $d$ & $0,021 \pm 0,004 \mathrm{~cd}$ & & & $0,616 \pm 0,064 b$ & $0,750 \pm 0,112 a b$ \\
\hline Mehriährige Blühmischung & $0,062 \pm 0,010$ & c & $0,055 \pm 0,008 b$ & b & & $0,405 \pm 0,078 \mathrm{c}$ & $0,320 \pm 0,044 \mathrm{~cd}$ \\
\hline Durchwachsene Silphie & n.v. & & $0,028 \pm 0,006$ & c & & n.v. & $0,331 \pm 0,032 \quad c$ \\
\hline \multirow[t]{3}{*}{ b) } & \multicolumn{7}{|c|}{ Schoningen } \\
\hline & \multicolumn{5}{|c|}{ Cobalt (mg kg $\left.{ }^{-1} \mathrm{TM}\right)$} & \multicolumn{2}{|c|}{ Nickel (mg kg-1 TM) } \\
\hline & 2015 & & 2016 & & & 2015 & 2016 \\
\hline Wickroggen & n.v. & & $0,037 \pm 0,010$ & $\mathrm{~b}$ & & n.v. & $0,269 \pm 0,107 \quad b c$ \\
\hline Winterackerbohnen & $0,095 \pm 0,038$ & $b$ & $0,198 \pm 0,035$ & a & * & $0,287 \pm 0,098 \quad b$ & $0,410 \pm 0,065 a b$ \\
\hline Wintertriticale & $0,011 \pm 0,003$ & $c$ & $0,011 \pm 0,002 b$ & $\mathrm{bc}$ & & $0,100 \pm 0,073 \mathrm{~cd}$ & $0,083 \pm 0,039 \quad e$ \\
\hline Gemenge WAB / WT & $0,082 \pm 0,021$ & $b$ & $0,144 \pm 0,042$ & a & * & $0,274 \pm 0,063 \quad b$ & $0,282 \pm 0,083 b c$ \\
\hline Sommerackerbohnen & $0,284 \pm 0,093$ & a & $0,254 \pm 0,060$ & a & & $0,601 \pm 0,163 \quad a$ & $0,456 \pm 0,053 \quad a$ \\
\hline Einjährige Blühmischung & $0,129 \pm 0,060$ & $b$ & $0,156 \pm 0,091$ & a & & $0,271 \pm 0,071 \quad b$ & $0,261 \pm 0,091 \mathrm{bcc}$ \\
\hline Amarant & $0,109 \pm 0,059$ & $b$ & $0,150 \pm 0,042$ & a & & $0,122 \pm 0,068 \mathrm{bcd}$ & $0,221 \pm 0,082 \mathrm{~cd}$ \\
\hline Mais & $0,012 \pm 0,007$ & $c$ & $0,003 \pm 0,003$ & c & & $0,071 \pm 0,021 \quad d$ & $0,091 \pm 0,009 \quad e$ \\
\hline Gemenge Amarant / Mais & $0,020 \pm 0,005$ & c & $0,008 \pm 0,005 b$ & & & $0,075 \pm 0,084 \quad d$ & $0,096 \pm 0,018 \mathrm{de}$ \\
\hline Deutsches Weidelgras & n.v. & & $0,034 \pm 0,016 b$ & & & n.v. & $0,415 \pm 0,110 a b$ \\
\hline Mehrjährige Blühmischung & $0,089 \pm 0,022$ & $b$ & $0,163 \pm 0,078$ & a & * & $0,256 \pm 0,057 \mathrm{bc}$ & $0,264 \pm 0,096$ bc \\
\hline Durchwachsene Silphie & n.v. & & $0,043 \pm 0,035 b$ & bc & & n.v. & $0,276 \pm 0,054 \quad b c$ \\
\hline
\end{tabular}

Dargestellt sind die Mittelwerte \pm Standardabweichung, Varianten mit gleichen Buchstaben an einem Standort innerhalb eines Jahres sind nicht signifikant verschieden, $p<0,05$ (Tukey-Test), ${ }^{*}$ kennzeichnen signifikante Unterschiede zwischen den Versuchsjahren innerhalb einer Variante an einem Standort, $p<0,05$ ( $\mathrm{t}-$ Test), n.v. = Werte nicht verfügbar. 


\subsection{Wurzeldaten}

Tabelle 12: Ergebnisse der statistischen Analyse (ANOVA) der festen Effekte Variante und Jahr sowie deren Interaktion im linearen gemischten Modell für die erfassten Wurzeldaten (Wurzel-Trockenmasse, C/N-Verhältnis der Wurzeln und N-Akkumulation in die unterirdische Biomasse) an den Standorten Reinshof und Schoningen.

\begin{tabular}{|c|c|c|c|c|c|c|}
\hline & \multicolumn{3}{|c|}{ Reinshof } & \multicolumn{3}{|c|}{ Schoningen } \\
\hline & F-Wert & $\operatorname{Pr}>\mathrm{F}$ & & F-Wert & $\operatorname{Pr}>\mathrm{F}$ & \\
\hline \multicolumn{7}{|c|}{ Wurzel-Trockenmasse } \\
\hline Variante & 36,83 & $<0,0001$ & $* * *$ & 55,59 & $<0,0001$ & $* \star *$ \\
\hline Jahr & 1,65 & 0,2043 & n.s. & 3,01 & 0,0876 & n.s. \\
\hline Variante $\mathrm{x}$ Jahr & 4,10 & 0,0002 & $* * *$ & 2,40 & 0,0249 & * \\
\hline \multicolumn{7}{|c|}{ C/N-Verhältnis der Wurzeln } \\
\hline Variante & 9,50 & $<0,0001$ & $* * *$ & 26,78 & $<0,0001$ & *** \\
\hline Jahr & 0,04 & 0,8343 & n.s. & 1,88 & 0,1760 & n.s. \\
\hline Variante $\mathrm{x}$ Jahr & 1,25 & 0,2796 & n.s. & 5,09 & $<0,0001$ & $* * *$ \\
\hline \multicolumn{7}{|c|}{ N-Akkumulation Wurzel } \\
\hline Variante & 60,64 & $<0,0001$ & $* * *$ & 55,62 & $<0,0001$ & *** \\
\hline Jahr & 0,06 & 0,8137 & n.s. & 0,96 & 0,3312 & n.s. \\
\hline Variante $\mathrm{x}$ Jahr & 4,18 & 0,0002 & $* * *$ & 2,62 & 0,0154 & * \\
\hline
\end{tabular}

Signifikanzniveau: ${ }^{* * *} p \leq 0,001 ;{ }^{* *} p \leq 0,01 ;{ }^{*} p \leq 0,05 ;$ n.s. $=$ nicht signifikant.

An den Standorten Reinshof und Schoningen gab es einen signifikanten Effekt der Variante auf die Wurzel-Trockenmasse, das $\mathrm{C} / \mathrm{N}$-Verhältnis der Wurzeln sowie die N-Akkumulation in den Wurzeln (Tabelle 12). Während das Jahr die Wurzelparameter an beiden Standorten nicht signifikant beeinflusste, war dies, bis auf das C/N-Verhältnis am Standort Reinshof, für die Interaktion von Variante und Jahr jedoch der Fall.

\subsubsection{Wurzel-Trockenmasse}

\section{Reinshof}

Im ersten Versuchsjahr blieben am Standort Reinshof in einer Tiefe von 0-60 cm nach der Ernte der Feldfrüchte Wurzel-Trockenmassen zwischen 2,9 dt TM ha-1 beim Amarant und 48,0 dt TM ha ${ }^{-1}$ beim Zweikulturnutzungssystem aus Winterackerbohnen als Erst- und Mais als Zweitfrucht im Boden zurück (Abbildung 12). Ebenfalls hohe Wurzeltrockenmassen erzielte das Zweikulturnutzungssystem aus dem Gemenge aus Winterackerbohnen und Wintertriticale und nachfolgendem Mais (43,8 dt TM ha-1), das Deutsche Weidelgras (42,2 dt TM ha-1) sowie die Sommerackerbohnen (37,6 dt TM ha $\left.{ }^{-1}\right)$. Mit 29,3 bzw. 25,5 dt TM ha-1 waren die Zweikulturnutzungssysteme aus Wickroggen sowie Wintertriticale jeweils gefolgt von Mais zwar unterhalb dieser Gruppe, unterscheiden sich aber dennoch nicht signifikant. Von diesen Varianten wiederum unterschied sich der Mais und von der Wintertriticale gefolgt von Mais auch das Gemenge aus Amarant und Mais nicht signifikant, wenngleich die Wurzel-Trockenmassen hier mit 12,2 bzw.10,1 dt TM ha-1 um einiges geringer waren.

Im Zweikulturnutzungssystem lag der Mais als Zweitfrucht mit Mengen von 8,6 dt TM ha-1 nach Wintertriticale bis 12,4 dt TM ha-1 nach Winterackerbohnen auf gleichem Niveau mit dem Mais als Sommerhauptfrucht. Damit trug der Mais mit einem Anteil von gut einem Drittel an der Gesamt-Wurzel-Trockenmasse bei den getreidedominierten Erstfrüchten (Wickroggen und Wintertriticale) und einem Viertel bei den Erstfrüchten mit Winterackerbohnenbeteiligung (Winterackerbohnen und Gemenge aus Winterackerbohnen und Wintertriticale) bei. 
Im zweiten Versuchsjahr waren die Unterschiede zwischen den Varianten bei den gebildeten Wurzel-Trockenmassen mit Werten zwischen 3,5 dt TM ha ${ }^{-1}$ beim Amarant und 63,4 dt TM ha $^{-1}$ beim Deutschen Weidelgras noch größer als im ersten Versuchsjahr (Abbildung 12). Hohe Wurzel-Trockenmassen hinterließen auch die Varianten des Zweikulturnutzungssystems aus Winterackerbohnen gefolgt von Mais und dem Gemenge aus Winterackerbohnen und Wintertriticale gefolgt von Mais sowie die Sommerackerbohnen mit 50,8, 42,4 bzw. 38,0 dt TM ha-1 im Boden und unterschieden sich damit nicht signifikant vom Deutschen Weidelgras. Eine weitere Gruppe bildeten die Varianten des Zweikulturnutzungssystems Wickroggen und Wintertriticale jeweils gefolgt von Mais sowie die einjährige Blühmischung mit Wurzel-Trockenmassen im Bereich von 27 bis $32 \mathrm{dt} \mathrm{TM} \mathrm{ha}^{-1}$. Aber auch die Durchwachsene Silphie war mit etwa 23 dt TM ha ${ }^{-1}$ hier einzuordnen. Der Amarant, der Mais in Reinsaat, das Gemenge aus Amarant und Mais sowie die mehrjährige Blühmischung blieben mit Wurzeltrockenmassen zwischen 3,5 und 7,2 dt TM ha ${ }^{-1}$ deutlich hinter diesen zurück.

Mais als Zweitfrucht hatte im zweiten Versuchsjahr, abgesehen von Mais nach Winterackerbohnen, höhere Wurzel-Trockenmassen als die Zweitfrucht im ersten Versuchsjahr. Damit trug er im zweiten Versuchsjahr mit bis zu zwei Drittel (Wickroggen + Mais, Wintertriticale + Mais) an der Gesamt-Wurzel-Trockenmasse bei. Trotz der Unterschiede zwischen Mais im ersten und zweiten Versuchsjahr unterschieden sich die Gesamt-Wurzel-Trockenmassen als Summe aus Erst- und Zweitfrucht aufgrund der z.T. deutlich geringeren Wurzel-Trockenmassen der Erstfrüchte im zweiten Versuchsjahr im Vergleich zum ersten nicht wesentlich zwischen den Versuchsjahren.

Deutlich stärker unterschieden sich die Versuchsjahre bei der einjährigen Blühmischung. Sie zeigte im zweiten Versuchsjahr mehr als die siebenfache Menge an Wurzel-Trockenmasse als im ersten Versuchsjahr. Zu einem signifikanten Jahreseffekt führte dies jedoch nicht. Eine deutliche Zunahme an Wurzeltrockenmasse vom ersten zum zweiten Versuchsjahr war auch beim mehrjährig angebauten Deutschen Weidelgras zu verzeichnen, wobei im zweiten Versuchsjahr $21 \mathrm{dt} \mathrm{TM} \mathrm{ha-1} \mathrm{mehr} \mathrm{an} \mathrm{Wurzel-Trockenmasse} \mathrm{zu} \mathrm{finden} \mathrm{waren,} \mathrm{als} \mathrm{im} \mathrm{ersten} \mathrm{Ver-}$ suchsjahr. 

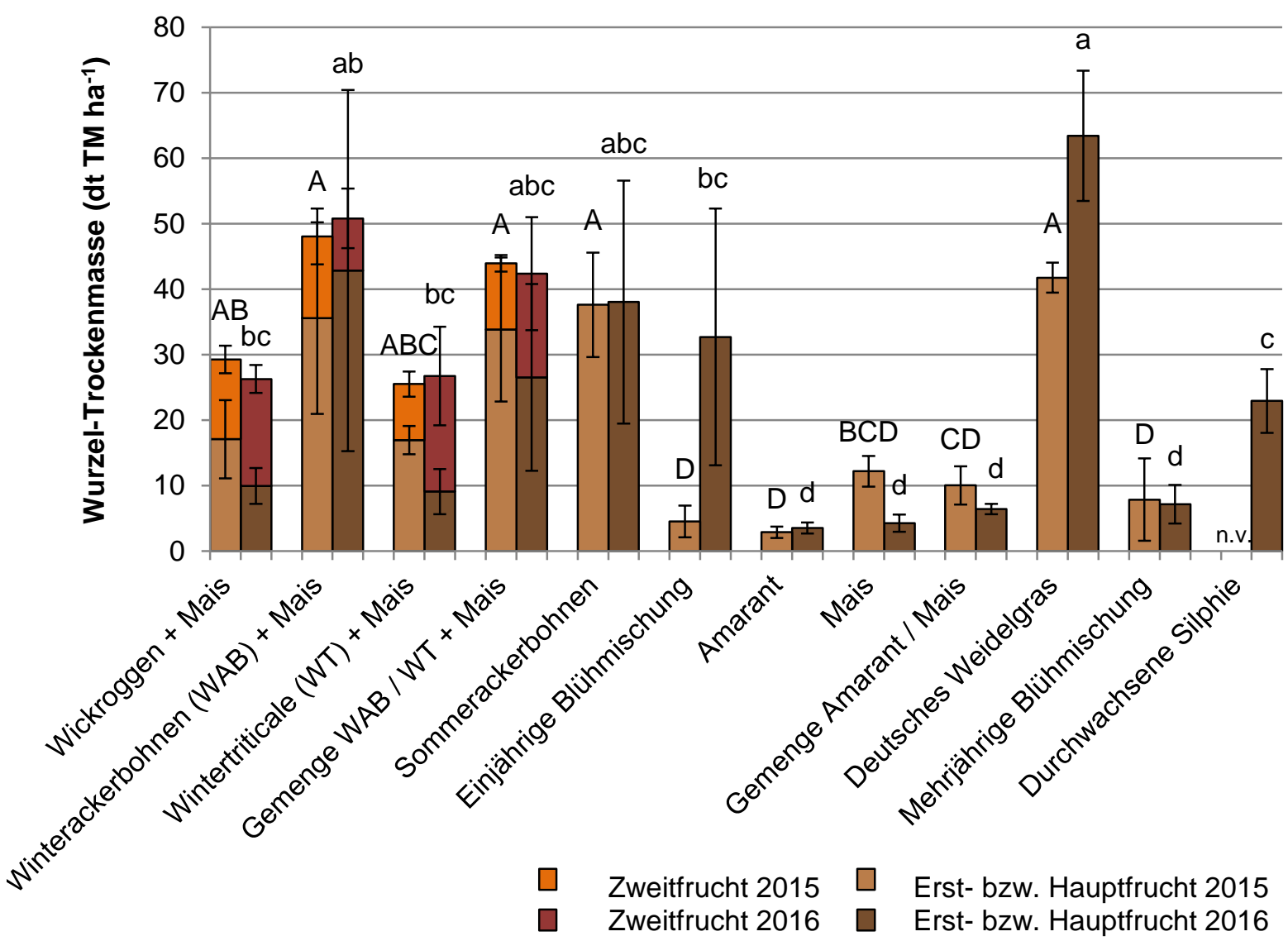

Abbildung 12: Trockenmasseerträge der Wurzeln in einer Tiefe von 0-60 cm in den Versuchsjahren 2015 und 2016 am Standort Reinshof. Dargestellt sind die Mittelwerte \pm Standardabweichung. Varianten mit gleichen Großbuchstaben bzw. gleichen Kleinbuchstaben sind nicht signifikant verschieden, $p<0,05$ (Tukey-Test). Bei gestapelten Säulen beziehen sich die Angaben auf die Höhe der Gesamtsäule. n.v. = Werte nicht verfügbar.

\section{Schoningen}

Am Standort Schoningen wurde die Wurzeltrockenmasse im Boden anders als am Standort Reinshof nur bis in eine Tiefe von $30 \mathrm{~cm}$ erfasst. Dennoch wurden auch an diesem Standort Wurzel-Trockenmassen bis zu $48 \mathrm{dt} \mathrm{TM} \mathrm{ha-1} \mathrm{im} \mathrm{Boden} \mathrm{zurückgelassen} \mathrm{(Abbildung} \mathrm{13).} \mathrm{Das}$ ist vergleichbar mit den am Standort Reinshof ermittelten Mengen.

Im ersten Versuchsjahr lagen die gebildeten Wurzel-Trockenmassen am Standort Schoningen zwischen 35,32 dt TM ha ${ }^{-1}$ beim Zweikulturnutzungssystem aus dem Gemenge Winterackerbohnen / Wintertriticale und nachfolgendem Mais und 2,35 dt TM ha ${ }^{-1}$ bei der mehrjährigen Blühmischung. Etwas geringere, aber nicht signifikant geringere Wurzeltrockenmassen im Vergleich zu dem Gemenge aus Winterackerbohnen und Wintertriticale gefolgt von Mais zeigten die übrigen im ersten Versuchsjahr an diesem Standort getesteten Varianten des Zweikulturnutzungssystems, Winterackerbohnen + Mais $\left(27,60 \mathrm{dt} \mathrm{TM} \mathrm{ha}^{-1}\right)$ sowie Wintertritcale + Mais $\left(21,55 \mathrm{dt} \mathrm{TM} \mathrm{ha}^{-1}\right)$, aber auch die Sommerackerbohnen $\left(24,25 \mathrm{dt} \mathrm{TM} \mathrm{ha}{ }^{-1}\right)$. Wie am Standort Reinshof im ersten Versuchsjahr auch, bildeten hier die ein- und mehrjährige Blühmischung sowie der Amarant eine Gruppe mit den geringsten Wurzeltrockenmassen im Bereich von etwa $2 \mathrm{dt}$ TM ha $^{-1}$. 
Bei der Zweitfrucht Mais wurden im ersten Versuchsjahr bei allen untersuchten Varianten des Zweikulturnutzungssystems Wurzel-Trockenmassen von etwa $10 \mathrm{dt} \mathrm{TM}^{-1}$ gemessen. Das war vergleichbar mit Mais als Sommerhauptfrucht mit 12,15 dt TM ha-1. Die Zweitfrucht trug damit zwischen einem Viertel beim Gemenge aus Winterackerbohnen und Wintertriticale und etwa der Hälfte bei Wintertriticale zur Gesamt-Wurzel-Trockenmasse dieser Varianten bei.

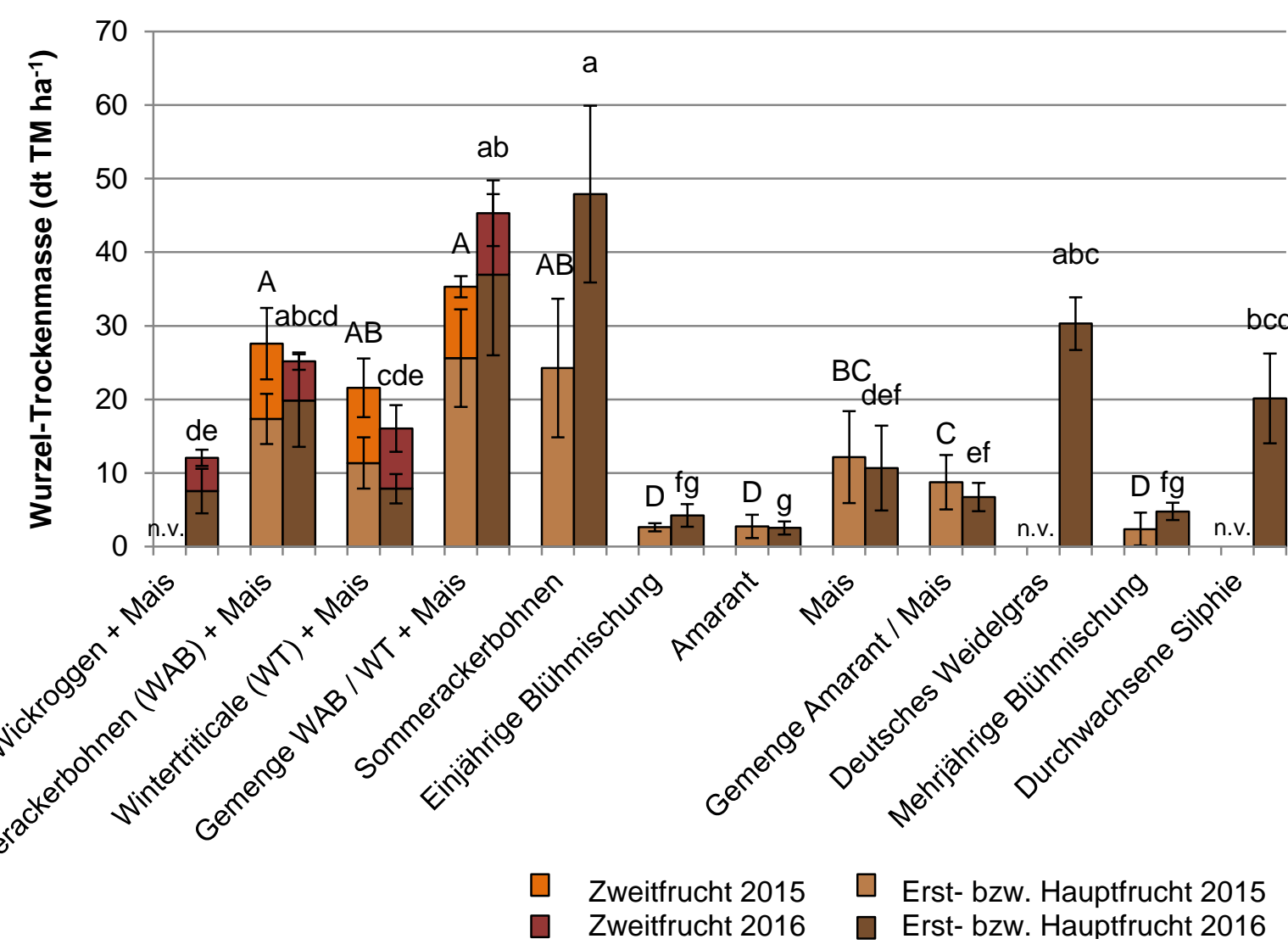

Abbildung 13: Trockenmasseerträge der Wurzeln in einer Tiefe von 0-30 cm in den Versuchsjahren 2015 und 2016 am Standort Schoningen. Dargestellt sind die Mittelwerte \pm Standardabweichung. Varianten mit gleichen Großbuchstaben bzw. gleichen Kleinbuchstaben sind nicht signifikant verschieden, $\mathrm{p}<0,05$ (Tukey-Test). Bei gestapelten Säulen beziehen sich die Angaben auf die Höhe der Gesamtsäule. n.v. = Werte nicht verfügbar.

Im zweiten Versuchsjahr hinterließen die Sommerackerbohnen große Wurzelmassen im Boden mit 47,92 dt TM ha-1. Nicht signifikant verschieden dazu waren das Gemenge aus Winterackerbohnen und Wintertriticale gefolgt von Mais mit 45,31 dt TM ha-1 aber, wie auch schon im ersten Versuchsjahr an diesem Standort bzw. am Standort Reinshof bei verschiedenen Varianten beobachtet, auch Varianten mit deutlich geringeren Wurzel-Trockenmassen. Hier betraf dies das Deutsche Weidelgras mit 30,30 dt TM ha ${ }^{-1}$ sowie die Variante Winterackerbohnen gefolgt von Mais mit 25,19 dt TM ha-1. Die Durchwachsene Silphie unterschied sich mit 20,1 dt TM ha-1 wiederum nicht signifikant von den letztgenannten Varianten, aber auch nicht vom Gemenge aus Winterackerbohnen und Wintertriticale gefolgt von Mais und auch nicht von Mais als Sommerhauptfrucht mit 10,6 dt TM ha-1. Klarer abgegrenzt werden konnten die Varianten mit den geringsten Wurzel-Trockenmassen. Das waren, wie im ersten Versuchsjahr auch, der Amarant sowie die ein- und mehrjährige Blühmischung mit Wurzeltrockenmassen von 2,53, 4,21 bzw. 4,77 dt TM ha-1. 
Der Mais als Zweitfrucht im Zweikulturnutzungssystem zeigte im zweiten Versuchsjahr sowohl geringere Wurzel-Trockenmassen als im ersten Versuchsjahr, aber auch im Vergleich zu Mais als Sommerhauptfrucht. Auffällig waren am Standort Schoningen die, anders als am Standort Reinshof, im Boden hinterlassenen hohen Wurzel-Trockenmassen des Gemenges aus Winterackerbohnen und Wintertriticale als Erstfrucht im Zweikulturnutzungssystem. Diese waren hier deutlich höher als bei Winterackerbohnen in Reinsaat.

Wie am Standort Reinshof auch, waren die deutlichen Unterschiede in den Wurzeltrockenmassen zwischen den Versuchsjahren bei einigen Varianten nicht signifikant. Sowohl bei den Sommerackerbohnen als auch der mehrjährigen Blühmischung konnten im zweiten Versuchsjahr etwa die doppelte Menge an Wurzeln gefunden werden als es im ersten Versuchsjahr der Fall war.

\subsubsection{C/N-Verhältnis der Wurzeln}

\section{Reinshof}

Das C/N-Verhältnis, das Aufschluss über die Humuswirksamkeit der im Boden zurückgelassenen Wurzeln geben soll, unterschied sich z.T. sehr stark zwischen den untersuchten Feldfrüchten. Im ersten Versuchsjahr hatten am Standort Reinshof der Mais und das Deutsche Weidelgras die weitesten C/N-Verhältnisse mit etwa 64 bzw. 62 (Tabelle 13). Signifikant verschieden davon waren die Varianten, bei denen Ackerbohnen enthalten waren. Das waren die Winterackerbohnen, das Gemenge aus Winterackerbohnen und Wintertriticale sowie die Sommerackerbohnen mit deutlich engeren $\mathrm{C} / \mathrm{N}-$ Verhältnissen von 26 bzw. 31. Die übrigen Varianten bewegten sich bei den C/N-Verhältnissen im Bereich von 42 bis 47 . Die im Boden zurückgebliebenen Wurzeln der Zweitfrucht Mais bei den Varianten des Zweikulturnutzungssystems wiesen im ersten Versuchsjahr C/N-Verhältnisse zwischen etwa 40 bei Mais nach dem Gemenge aus Winterackerbohnen und Wintertriticale und 45 bei Mais nach Winterackerbohnen bzw. nach Wintertriticale auf (Daten nicht dargestellt).

Im zweiten Versuchsjahr war das weiteste C/N-Verhältnis mit etwa 68 bei der einjährigen Blühmischung zu finden. Signifikant war der Unterschied jedoch nur zu den Varianten mit den engsten $\mathrm{C} / \mathrm{N}$-Verhältnissen, Winter- und Sommerackerbohnen mit jeweils 30. Signifikant verschieden zu den Ackerbohnenvarianten waren auch der Mais und das Gemenge aus Amarant und Mais mit $\mathrm{C} / \mathrm{N}-$ Verhältnissen von 49 bzw. 54. Alle anderen Varianten ordneten sich mit Werten von 33 bis 47 zwischen diesen ein. Die Zweitfrucht Mais im Zweikulturnutzungssystem hatte im zweiten Versuchsjahr weitere $\mathrm{C} / \mathrm{N}$-Verhältnisse als im ersten, zwischen etwa 50 bei Mais nach Winterackerbohnen und 67 bei Mais nach Wickroggen (Daten nicht dargestellt).

Ein großer Unterschied zwischen den Versuchsjahren zeigte sich bei der einjährigen Blühmischung sowie beim Deutschen Weidelgras, jedoch war dieser Unterschied nicht signifikant. Die Richtung der Veränderung war bei diesen Vaiante jedoch gegenläufig: die einjährige Blühmischung nahm vom ersten zum zweitem Versuchsjahr deutlich zu, das Deutsche Weidelgras deutlich ab. 
Tabelle 13: C/N-Verhältnis der Wurzeln (0-60 cm Tiefe) in den Versuchsjahren 2015 und 2016 am Standort Reinshof.

\begin{tabular}{|c|c|c|}
\hline Variante & $\begin{array}{c}2015 \\
\text { (C/N Verhältnis) }\end{array}$ & $\begin{array}{c}2016 \\
\text { (C/N Verhältnis) }\end{array}$ \\
\hline Wickroggen & $47,31 \pm 7,13 \quad a b$ & $45,20 \pm 5,80 \quad a b$ \\
\hline Winterackerbohnen (WAB) & $25,71 \pm 5,31 \quad c$ & $29,82 \pm 4,84 \quad b$ \\
\hline Wintertriticale (WT) & $42,02 \pm 2,49 \quad a b$ & $38,37 \pm 4,64 \quad a b$ \\
\hline Gemenge WAB / WT & $31,08 \pm 2,07 \quad b c$ & $33,40 \pm 3,37 \quad a b$ \\
\hline Sommerackerbohnen & $31,36 \pm 3,21 \quad b c$ & $30,17 \pm 6,18 \quad b$ \\
\hline Einjährige Blühmischung & $42,38 \pm 6,86 \quad a b$ & $67,79 \pm 44,35$ a \\
\hline Amarant & $43,35 \pm 7,30 \quad a b$ & $45,93 \pm 21,94 a b$ \\
\hline Mais & $64,08 \pm 23,20 \quad a$ & $49,36 \pm 5,50 \quad a$ \\
\hline Gemenge Amarant / Mais & $43,64 \pm 9,16 \quad a b$ & $54,23 \pm 9,39 \quad a$ \\
\hline Deutsches Weidelgras & $62,28 \pm 5,42 \quad a$ & $43,11 \pm 10,68 a b$ \\
\hline Mehrjährige Blühmischung & $47,29 \pm 7,42 \quad a b$ & $47,14 \pm 7,66 \quad a b$ \\
\hline Durchwachsene Silphie & n.v. & $35,72 \pm 5,47 \quad a b$ \\
\hline
\end{tabular}

Dargestellt sind die Mittelwerte \pm Standardabweichung, Varianten mit gleichen Buchstaben innerhalb eines Jahres sind nicht signifikant verschieden, $p<0,05$ (Tukey-Test), n.v. = Werte nicht verfügbar.

\section{Schoningen}

Am Standort Schoningen unterschieden sich die C/N-Verhältnisse der Wurzeln mit Werten zwischen 20 bei Winterackerbohnen und 73 beim Gemenge aus Amarant und Mais jeweils im zweiten Versuchsjahr noch stärker zwischen den Varianten als am Standort Reinshof (Tabelle 14). Im ersten Versuchsjahr hatte das Gemenge aus Amarant und Mais das weiteste $\mathrm{C} / \mathrm{N}$ Verhältnis mit 57. Signifikante Unterschiede gab es, wie am Standort Reinshof auch, zu den Varianten, die Ackerbohnen enthielten. Diese hatten mit 28 bei Winterackerbohnen, 33 beim Gemenge aus Winterackerbohnen und Wintertriticale sowie 36 bei Sommerackerbohnen deutlich engere $\mathrm{C} / \mathrm{N}$-Verhältnisse. In diese Gruppe ließ sich hier auch die mehrjährige Blühmischung mit einem $\mathrm{C} / \mathrm{N}-$ Verhältnis von 34 einordnen.

Im zweiten Versuchsjahr hatte der Amarant mit 72 ein ähnlich weites $\mathrm{C} / \mathrm{N}$-Verhältnis wie das Gemenge aus Amarant und Mais. Wesentlich engere $\mathrm{C} / \mathrm{N}-$ Verhältnisse wurden wiederum bei den Wurzeln der Varianten mit Beteiligung von Ackerbohnen gefunden. Auf gleichem Niveau lag auch die Durchwachsene Silphie (30).

Signifikante Jahreseffekte gab es auch am Standort Schoningen nicht. So waren aber dennoch die C/N-Verhältnisse bei den Winter- und Sommerackerbohnen im zweiten Versuchsjahr niedriger als im ersten. Beim Amarant, dem Mais und der mehrjährigen Blühmischung war der Effekt genau anders herum. Hier waren die C/N-Verhältnisse im zweiten Versuchsjahr weiter als im ersten. 
Tabelle 14: C/N-Verhältnis der Wurzeln (0-30 cm Tiefe) in den Versuchsjahren 2015 und 2016 am Standort Schoningen.

\begin{tabular}{llrlrl}
\hline \multirow{2}{*}{ Variante } & \multicolumn{2}{c}{2015} & \multicolumn{2}{c}{2016} \\
Wickroggen & n.v. & & $47,88 \pm 5,74$ & bcd \\
Winterackerbohnen (WAB) & $27,66 \pm 4,16$ & d & $19,81 \pm 0,57$ & $f$ & (C/N Verhälthis) \\
Wintertriticale (WT) & $47,66 \pm 8,48$ & abc & $40,36 \pm 5,49$ & de \\
Gemenge WAB / WT & $32,96 \pm 8,34$ & cd & $29,39 \pm 0,95$ & ef \\
Sommerackerbohnen & $35,85 \pm 8,75$ & bcd & $26,53 \pm 2,42$ & $\mathrm{f}$ \\
Einjährige Blühmischung & $42,74 \pm 16,53$ abcd & $50,21 \pm 6,25$ & abcd \\
Amarant & $53,37 \pm 12,44$ & ab & $72,02 \pm 24,80$ & ab \\
Mais & $49,45 \pm 3,10$ & ab & $66,72 \pm 12,60$ & abc \\
Gemenge Amarant / Mais & $57,48 \pm 4,38$ & a & $72,65 \pm 8,78$ & a \\
Deutsches Weidelgras & n.v. & & $44,02 \pm 3,02$ & de \\
Mehrjährige Blühmischung & $33,36 \pm 7,39$ & cd & $46,83 \pm 8,92$ & cd \\
Durchwachsene Silphie & n.v. & & $29,71 \pm 3,65$ & ef \\
\hline
\end{tabular}

Dargestellt sind die Mittelwerte \pm Standardabweichung, Varianten mit gleichen Buchstaben innerhalb eines Jahres sind nicht signifikant verschieden, $p<0,05$ (Tukey-Test), n.v. = Werte nicht verfügbar.

\subsubsection{Stickstoff in der Wurzel-Trockenmasse}

\section{Reinshof}

In einer Tiefe von 0-60 $\mathrm{cm}$ reichten sie hier von $3 \mathrm{~kg} \mathrm{~N}^{-1}$ beim Amarant im ersten Versuchsjahr bis $75 \mathrm{~kg} \mathrm{~N}$ ha-1 $^{-1}$ beim Zweikulturnutzungssystem aus Winterackerbohnen gefolgt von Mais im zweiten Versuchsjahr (Abbildung 14).

Nicht viel weniger Stickstoff als dies nahmen die Winterackerbohnen und der nachfolgende Mais mit $74 \mathrm{~kg} \mathrm{~N}$ ha $^{-1}$ hier auch im ersten Versuchsjahr auf. Ebenfalls hohe Stickstoffmengen in der Wurzelbiomasse zeigten das Zweikulturnutzungssystem aus dem Gemenge aus Winterackerbohnen und Wintertriticale gefolgt von Mais sowie die Sommerackerbohnen. Mit 56

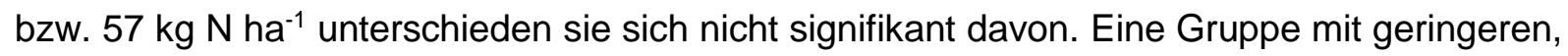
wenn auch nicht signifikant geringeren N-Mengen in der Wurzelbiomasse, waren im ersten Versuchsjahr die getreidebetonten Erstfrüchte Wickroggen und Wintertriticale gefolgt von Mais sowie das Deutsche Weidelgras mit Werten zwischen 26 und $30 \mathrm{~kg} \mathrm{~N} \mathrm{ha}^{-1}$. Signifikant geringer waren die in der Wurzelbiomasse gespeicherten N-Mengen bei den Blühmischungen und den Sommerhauptfrüchten Amarant, Mais und dem Gemenge aus Amarant und Mais mit Werten von höchstens $10 \mathrm{~kg} \mathrm{~N} \mathrm{ha}^{-1}$.

Im zweiten Versuchsjahr zeigten wieder die Varianten, bei denen Ackerbohnen im Anbau waren, also Winterackerbohnen sowie das Gemenge aus Winterackerbohnen und Wintertriticale gefolgt von Mais und die Sommerackerbohnen, mit 46 bis $75 \mathrm{~kg} \mathrm{~N} \mathrm{ha}^{-1}$ die höchsten N-Mengen in der Wurzelbiomasse. Ein Unterschied bestand hier jedoch beim Deutschen Weidelgras. Mit

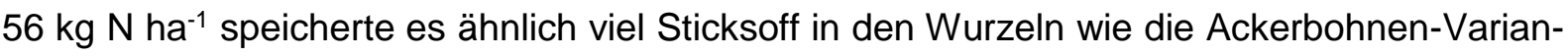
ten. Geringer aber dennoch nicht signifikant geringer als diese Varianten war die Durchwachsene Silphie mit etwa $30 \mathrm{~kg} \mathrm{~N} \mathrm{ha}^{-1}$. Wesentlich weniger Stickstoff akkumulierten auch in diesem Versuchsjahr wieder die Blühmischungen sowie der Amarant, der Mais und das Gemenge 
aus beiden. Durch den Mais als Zweitfrucht wurden vor allem im zweiten Versuchsjahr höhere $\mathrm{N}-$ Mengen mit den Wurzeln eingetragen als durch den Mais als Sommerhauptfrucht.

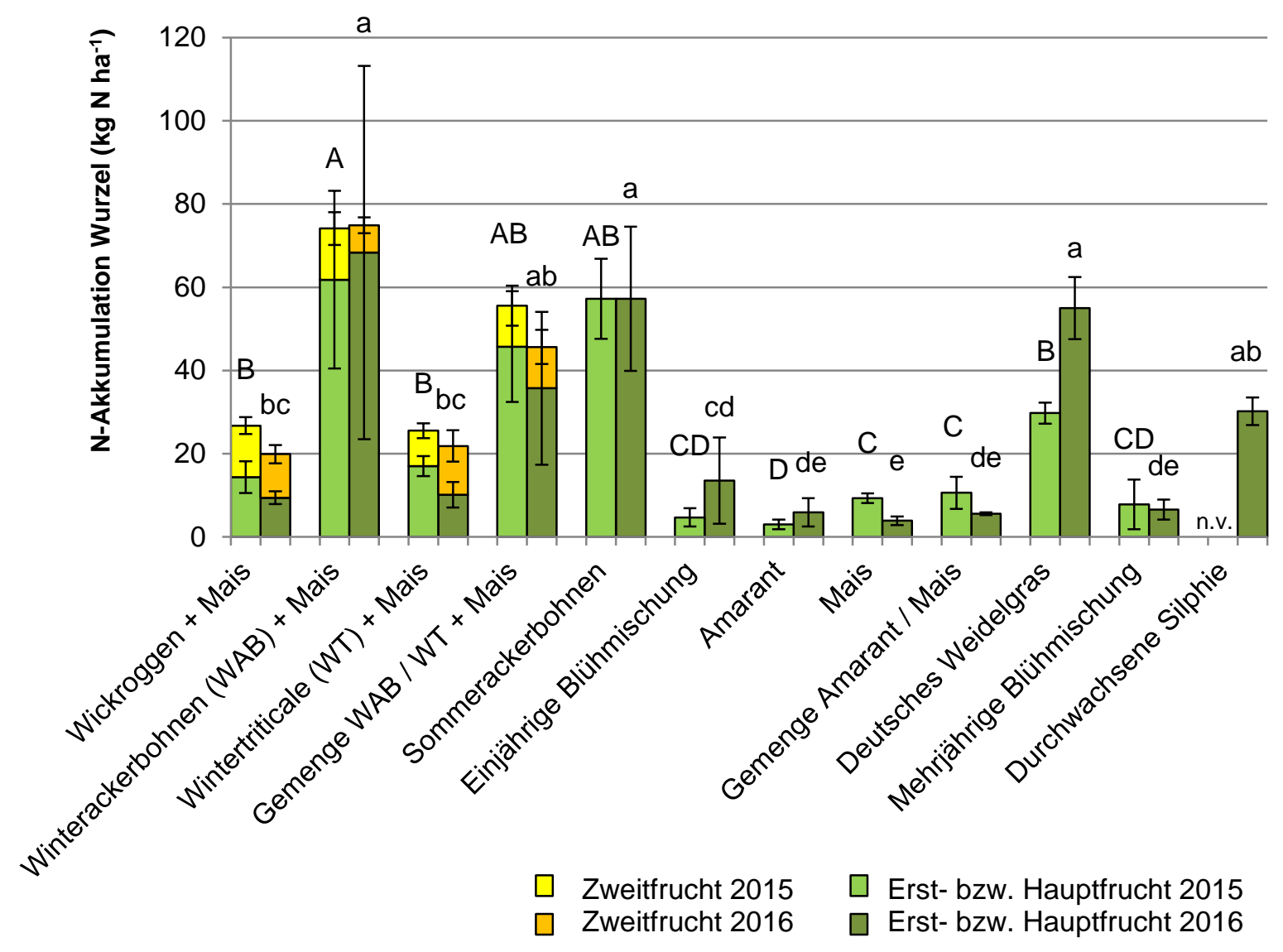

Abbildung 14: Stickstoffakkumulation in der unterirdischen Biomasse $(0-60 \mathrm{~cm})$ in den Versuchsjahren 2015 und 2016 am Standort Reinshof. Dargestellt sind die Mittelwerte \pm Standardabweichung. Varianten mit gleichen Großbuchstaben bzw. gleichen Kleinbuchstaben sind nicht signifikant verschieden, $p<0,05$ (Tukey-Test). Bei gestapelten Säulen beziehen sich die Angaben auf die Höhe der Gesamtsäule. n.v. = Werte nicht verfügbar.

\section{Schoningen}

Die in den Wurzeln gespeicherten N-Mengen variierten auch an diesem Standort sehr stark zwischen den Varianten und reichten in $0-30 \mathrm{~cm}$ Tiefe von $2 \mathrm{~kg} \mathrm{~N}^{-1}$ bei Amarant im ersten

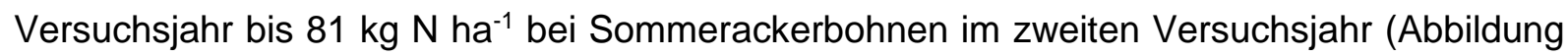
15).

Im ersten Versuchsjahr wurden am Standort Schoningen wie am Standort Reinshof auch, die größten N-Mengen in der Wurzelbiomasse der Varianten gemessen, bei denen Ackerbohnen beteiligt waren. So unterschieden sich das Gemenge aus Winterackerbohnen und Wintertriticale gefolgt von Mais (48 $\left.\mathrm{kg} \mathrm{N}^{-1}\right)$, die Winterackerbohnen gefolgt von Mais (40 kg N ha-1) und die Sommerackerbohnen (34 $\mathrm{kg} \mathrm{N} \mathrm{ha}^{-1}$ ) nicht signifikant voneinander, aber auch nicht von der Getreide-Variante Wintertriticale gefolgt von Mais (21 kg N ha-1). Die übrigen Varianten hinterließen mit ihren Wurzeln wesentlich weniger Stickstoff im Boden. 

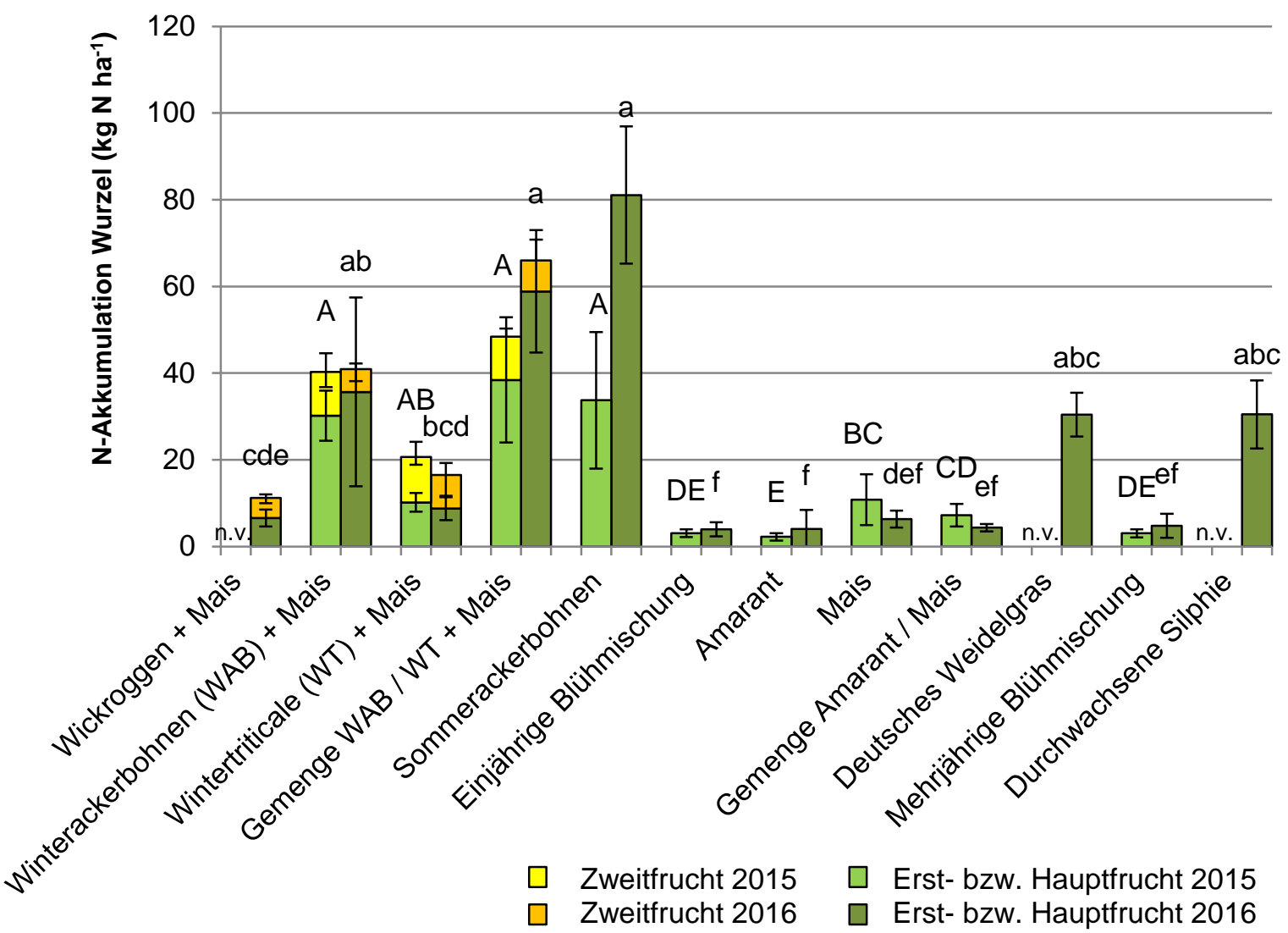

Abbildung 15: Stickstoffakkumulation in der unterirdischen Biomasse $(0-30 \mathrm{~cm})$ in den Versuchsjahren 2015 und 2016 am Standort Schoningen. Dargestellt sind die Mittelwerte \pm Standardabweichung. Varianten mit gleichen Großbuchstaben bzw. gleichen Kleinbuchstaben sind nicht signifikant verschieden, $p<0,05$ (Tukey-Test). Bei gestapelten Säulen beziehen sich die Angaben auf die Höhe der Gesamtsäule. n.v. = Werte nicht verfügbar.

Auffällig im zweiten Versuchsjahr waren die Sommerackerbohnen, die mit $81 \mathrm{~kg} \mathrm{~N}^{\mathrm{N}} \mathrm{a}^{-1} \mathrm{nicht}$ nur an diesem Standort die Variante mit der größten N-Menge in den Wurzeln waren, sondern trotz der geringeren Beprobungstiefe auch die akkumulierten N-Mengen am Standort Reinshof übertrafen. Allerdings waren auch Varianten mit deutlich geringeren N-Mengen im Bereich von $66 \mathrm{~kg} \mathrm{~N} \mathrm{ha}^{-1}$, wie beim Gemenge aus Winterackerbohnen und Wintertriticale und folgendem

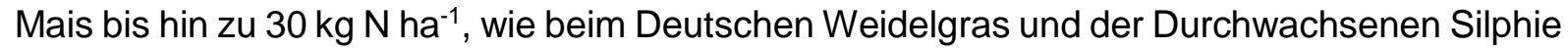
nicht signifikant verschieden davon. Trotz der hohen N-Mengen bei den Sommerackerbohnen im zweiten Versuchsjahr unterschieden sie sich nicht signifikant vom ersten Versuchsjahr.

\subsection{Bodendaten}

Am Standort Reinshof gab es in beiden Versuchsjahren sowohl beim Nmin im Winter als auch nach der Ernte einen hoch signifikanten Effekt der Variante (Tabelle 15). Am Standort Schoningen war dies für den Nmin nach der Ernte ebenso der Fall. Beim Nmin im Winter hingegen gab es hier nur im zweiten Versuchsjahr einen signifikanten Effekt. Die Menge an pflanzenverfügbarem Wasser im Boden wurde keiner statistischen Analyse unterzogen. 
Tabelle 15: Ergebnisse der statistischen Analyse (ANOVA) der festen Effekts Variante im linearen gemischten Modell für den erfassten Parameter Nmin an den Standorten Reinshof und Schoningen in den Versuchsjahren 2015 und 2016.

\begin{tabular}{|c|c|c|c|c|c|c|}
\hline & \multicolumn{3}{|c|}{ Reinshof } & \multicolumn{3}{|c|}{ Schoningen } \\
\hline & F-Wert & $\operatorname{Pr}>\mathrm{F}$ & & F-Wert & $\operatorname{Pr}>\mathrm{F}$ & \\
\hline $\begin{array}{l}\text { Nmin im Winter } \\
\text { Variante Jahr } 2015 \\
\text { Jahr } 2016\end{array}$ & $\begin{array}{l}43,95 \\
13,92\end{array}$ & $\begin{array}{l}<0,0001 \\
<0,0001\end{array}$ & $\begin{array}{l}* \star * \\
* \star *\end{array}$ & $\begin{array}{l}0,46 \\
4,85\end{array}$ & $\begin{array}{l}0,7170 \\
0,0022\end{array}$ & $\underset{* *}{\text { n.s. }}$ \\
\hline $\begin{array}{l}\text { Nmin nach Ernte } \\
\text { Variante Jahr } 2015 \\
\text { Jahr } 2016\end{array}$ & $\begin{array}{l}22,76 \\
57,01\end{array}$ & $\begin{array}{l}<0,0001 \\
<0,0001\end{array}$ & $\begin{array}{l}* * * \\
\star \star *\end{array}$ & $\begin{array}{l}9,57 \\
14,83\end{array}$ & $\begin{array}{l}<0,0001 \\
<0,0001\end{array}$ & $\begin{array}{l}* * * \\
* \star \star\end{array}$ \\
\hline
\end{tabular}

Signifikanzniveau: ${ }^{* * *} p \leq 0,001 ;{ }^{* *} p \leq 0,01 ;{ }^{*} p \leq 0,05 ;$ n.s. $=$ nicht signifikant.

\subsubsection{Nmin-Mengen}

Reinshof

Erstes Versuchsjahr

Zur Aussaat der Erstfrüchte des Zweikulturnutzungssystems und des mehrjährig angebauten Deutschen Weidelgrases im Oktober 2014 fand die erste Erfassung der Nmin-Mengen im Boden statt. Im Mittel über die gesamte Versuchsanlage waren das in der Tiefe von 0-90 cm 45 $\mathrm{kg} \mathrm{N} \mathrm{ha}^{-1}$, wobei etwa die Hälfte des Stickstoffs im oberen Bodenabschnitt $(0-30 \mathrm{~cm})$, etwa $40 \%$ im mittleren $(30-60 \mathrm{~cm})$ und etwa $10 \%$ im unteren Bodenabschnitt $(60-90 \mathrm{~cm})$ zu finden war (Daten nicht dargestellt).

Bei der Erhebung der Nmin-Mengen im Winter des ersten Versuchsjahres ließen sich zwei Gruppen voneinander abgrenzen (Abbildung 16 a). Einmal waren es die Varianten, die im Winter höhere Werte zeigten als zur Aussaat und einmal die, die den Stickstoff von der Aussaat bis zum Winter absenken konnten. Zur ersten Gruppe zählten die Winterackerbohnen als Erstfrüchte im Zweikulturnutzungssystem und das Deutsche Weidelgras mit 54 bzw. $51 \mathrm{~kg} \mathrm{~N}$ $\mathrm{ha}^{-1}$. Damit unterschieden sich diese Varianten auch nicht signifikant von der Schwarzbrache mit $66 \mathrm{~kg} \mathrm{~N} \mathrm{ha}^{-1}$. Die Erstfrüchte des Zweikulturnutzungssystems Wickroggen und Wintertriticale hingegen konnten den zur Aussaat vorhanden Stickstoff stark absenken auf 15 bzw. 12 $\mathrm{kg} \mathrm{N} \mathrm{ha}^{-1}$. Das Gemenge aus Winterackerbohnen und Wintertriticale als Erstfrucht hinterließ zwar etwas mehr Stickstoff im Boden als der Wickroggen und die Wintertriticale, signifikant war dieser Unterschied jedoch nicht.

Auffällig bei den Nmin-Werten im Winter des ersten Versuchsjahres war die Aufteilung des Stickstoffs in die drei untersuchten Bodenabschnitte. Während der obere Bodenabschnitt bei allen untersuchten Varianten ähnlich war, nahmen im Vergleich zum Ausgangs-Nmin zur Aussaat bei 


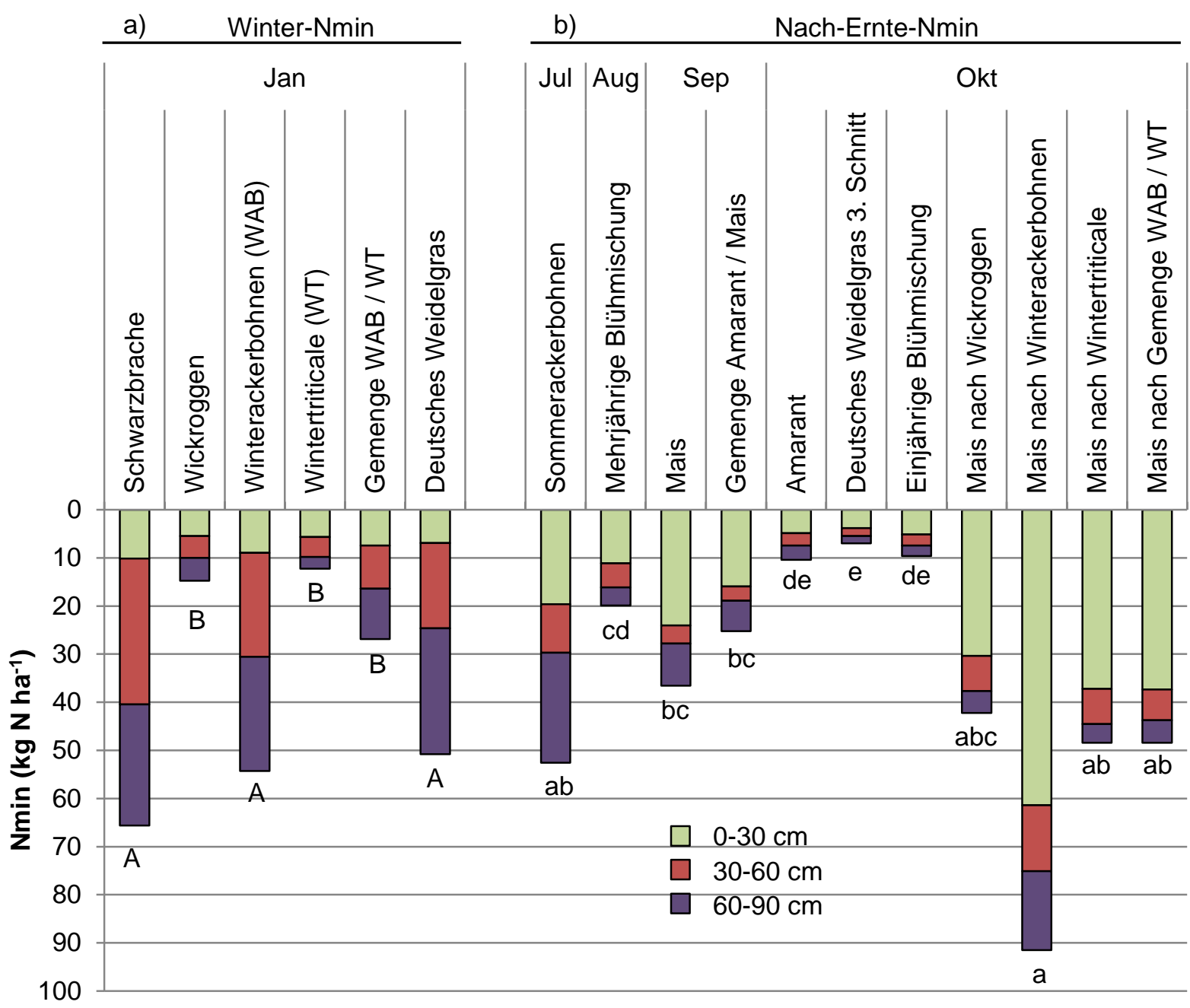

Abbildung 16: Nmin-Mengen in drei Tiefenstufen im Versuchsjahr 2015 am Standort Reinshof unterteilt in die Probenahme im Winter (a) und nach der Ernte im Sommer bzw. Herbst (b). Varianten mit gleichen Großbuchstaben (Winter-Nmin) bzw. gleichen Kleinbuchstaben (Nach-Ernte-Nmin) sind nicht signifikant verschieden, $\mathrm{p}<0,05$ (Tukey-Test). Die statistischen Angaben beziehen sich auf die Höhe der Gesamtsäule.

den Winterackerbohnen und dem Deutschen Weidelgras insbesondere die Nmin-Mengen in den unteren Bodenabschnitten zu. Das war die gleiche Entwicklung wie bei der Schwarzbrache.

Bei der Untersuchung des Bodens nach der Ernte der Feldfüchte des ersten Versuchsjahres fiel besonders der Mais als Zweitfrucht nach Winterackerbohnen des Zweikulturnutzungssystems auf (Abbildung $16 \mathrm{~b}$ ). Mit mehr als $91 \mathrm{~kg} \mathrm{~N}^{-1}$ blieben hier große Mengen mineralischen Stickstoffs in Boden zurück. Deutlich weniger, aber dennoch nicht signifikant weniger, residualer Stickstoff war bei den anderen Varianten des Zweikulturnutzungssystems mit $42 \mathrm{~kg} \mathrm{~N}$ ha $^{-1}$ nach der Ernte von Mais nach Wickroggen bzw. $48 \mathrm{~kg} \mathrm{~N} \mathrm{ha}^{-1}$ nach Wintertriticale und dem Gemenge aus Winterackerbohnen und Wintertriticale sowie nach Sommerackerbohnen mit etwa $53 \mathrm{~kg} \mathrm{~N} \mathrm{ha}^{-1}$ zu finden. Abgesehen von Mais nach Winterackerbohnen, waren auch die Nmin-Mengen von Mais als Sommerhauptfrucht sowie dem Gemenge aus Amarant und Mais nicht signifikant verschiedenen von den Nmin-Mengen nach Mais als Zweitfrucht. Sehr geringe Nmin-Mengen nach der Ernte hinterließen der Amarant $\left(10 \mathrm{~kg} \mathrm{~N} \mathrm{ha}^{-1}\right)$, das Deutsche Weidelgras ( $\left.7 \mathrm{~kg} \mathrm{~N} \mathrm{ha}^{-1}\right)$ und die einjährige Blühmischung $\left(10 \mathrm{~kg} \mathrm{~N} \mathrm{ha}^{-1}\right)$. Bei nahezu allen Varianten 
lag der Stickstoff bei diesem Erntetermin überwiegend im oberen Bodenabschnitt vor. Besonders auffällig war dies bei den Varianten des Zweikulturnutzungssystems.

\section{Reinshof}

Zweites Versuchsjahr

Im zweiten Versuchsjahr gingen in die Winter-Nmin-Untersuchungen neben dem Deutschen Weidelgras auch die übrigen mehrjährigen Varianten ein (Abbildung $17 \mathrm{a}$ ). Mit etwa $10 \mathrm{~kg} \mathrm{~N}$

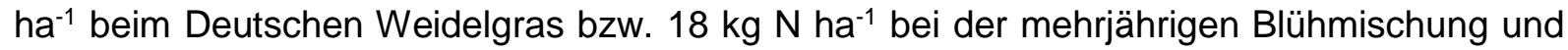
der Durchwachsenen Silphie befanden sich hier nur geringe Mengen mineralischen Stickstoffs im Boden. Größere Mengen konnten zu diesem Zeitpunkt hingegen bei den Erstfrüchten des Zweikulturnutzungssystems gefunden werden. Signifikant waren die Unterschiede zur mehrjährigen Blühmischung und der Durchwachsenen Silphie jedoch nicht in jedem Fall. Auch im zweiten Versuchsjahr waren innerhalb der Erstfrüchte des Zweikulturnutzungssystems die Winterackerbohnen die Variante mit den höchsten Nmin-Mengen im Winter. Mit $51 \mathrm{~kg} \mathrm{~N}^{\mathrm{N}} \mathrm{ha}^{-1}$ wiesen sie eine vergleichbare N-Menge im Boden auf, wie die Schwarzbrache mit $52 \mathrm{~kg} \mathrm{~N}$ ha $^{-1}$. Anders als im ersten Versuchsjahr, waren die Erstfrüchte im zweiten Versuchsjahr nicht in der Lage, den zur Aussaat im Oktober 2015 vorhandenen Stickstoff von $39 \mathrm{~kg} \mathrm{~N} \mathrm{ha}^{-1}$ bis zum Winter wesentlich abzusenken (Daten nicht dargestellt). Während zur Aussaat noch etwa $60 \%$ des Stickstoffs im oberen Bodenabschnitt $(0-30 \mathrm{~cm})$ vorlagen und nur etwa $10 \% \mathrm{im}$ unteren (60-90 cm), war es bei der Beprobung im Winter umgekehrt. Hier war der Großteil im unteren Bodenabschnitt zu finden.

Bei der Nmin-Beprobung nach der Ernte der Feldfrüchte im zweiten Versuchsjahr zeigte sich ein ähnliches Bild wie im ersten Versuchsjahr (Abbildung 17 b). Die Mais-Varianten, unabhängig ob als Sommerhauptfrucht, im Gemenge oder als Zweitfrucht, hinterließen mit Werten zwischen $42 \mathrm{~kg} \mathrm{~N}^{-1}$ bei Mais nach Wintertriticale und $63 \mathrm{~kg} \mathrm{~N}^{-1}$ bei Mais nach Winterackerbohnen, den meisten Stickstoff im Boden. Die Blühmischungen und der Amarant wiederum, aber auch die Durchwachsene Silphie hingegen zeigten nur sehr geringe N-Mengen im Bereich von 9-10 kg N ha-1. Noch weniger war beim Deutschen Weidelgras mit $4 \mathrm{~kg} \mathrm{~N}$ $\mathrm{ha}^{-1} \mathrm{zu}$ finden. Eine ebenfalls wiederkehrende Beobachtung aus dem ersten Versuchsjahr war die Aufteilung des Stickstoffs in die drei Bodenabschnitte. Auch im zweiten Versuchsjahr lag er bei den Mais-Varianten vor allem in den oberen $30 \mathrm{~cm}$ vor. 


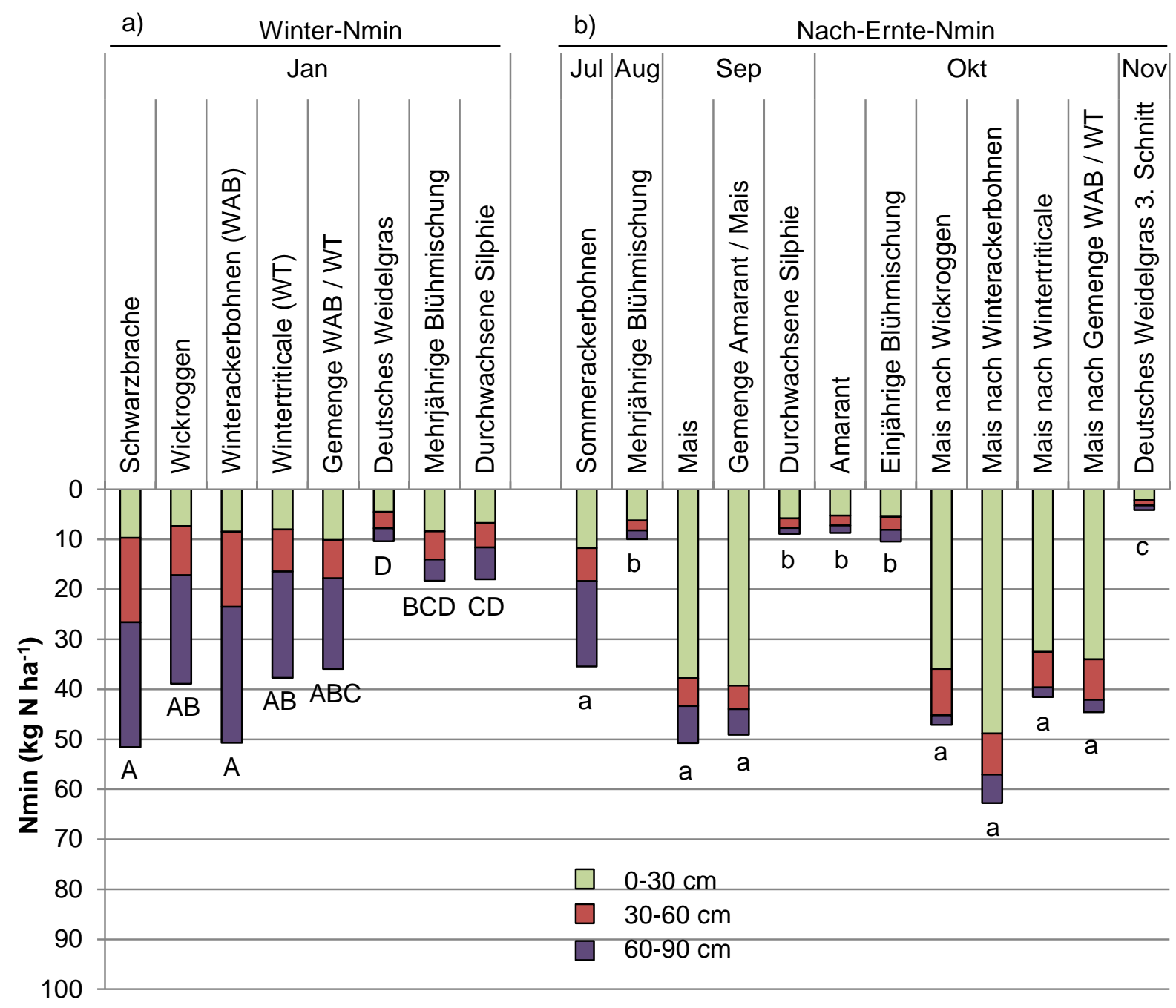

Abbildung 17: Nmin-Mengen in drei Tiefenstufen im Versuchsjahr 2016 am Standort Reinshof unterteilt in die Probenahme im Winter (a) und nach der Ernte im Sommer bzw. Herbst (b). Varianten mit gleichen Großbuchstaben (Winter-Nmin) bzw. gleichen Kleinbuchstaben (Nach-Ernte-Nmin) sind nicht signifikant verschieden, $p<0,05$ (Tukey-Test). Die statistischen Angaben beziehen sich auf die Höhe der Gesamtsäule.

\section{Schoningen}

\section{Erstes Versuchsjahr}

Die Nmin-Untersuchung im Winter des ersten Versuchsjahres am Standort Schoningen erbrachte keine signifikanten Unterschiede sowohl zwischen den Erstfrüchten des Zweikulturnutzungssystems als auch zwischen den Erstfrüchten und der Schwarzbrache (Abbildung 18a). Mit Werten zwischen $43 \mathrm{~kg} \mathrm{~N}$ ha $^{-1}$ bei der Wintertriticale und $52 \mathrm{~kg} \mathrm{~N} \mathrm{ha}^{-1}$ bei den Winterackerbohnen bzw. der Schwarzbrache waren sich diese Varianten relativ ähnlich. Zur Aussaat der Erstfrüchte im Oktober 2014 lagen im Mittel über die Versuchsanlage etwa $80 \mathrm{~kg} \mathrm{~N} \mathrm{ha}^{-1}$ vor (Daten nicht dargestellt). Das waren deutlich größere Mengen als im Winter unter den Erstfrüchten zu finden waren, aber auch im Vergleich zur Schwarzbrache. Hinsichtlich der Aufteilung in die drei Bodenabschnitte hat es von der Aussaat bis zum Winter überwiegend eine Veränderung im oberen Bodenabschnitt $(0-30 \mathrm{~cm})$ gegeben. Hier waren zur Aussaat etwa 50 $\mathrm{kg} \mathrm{N} \mathrm{ha}^{-1}$ vorhanden (Daten nicht dargestellt). Die N-Mengen in den Bodenabschnitten 30-60 $\mathrm{cm}$ und $60-90 \mathrm{~cm}$ blieben etwa gleich. 
a) Winter-Nmin

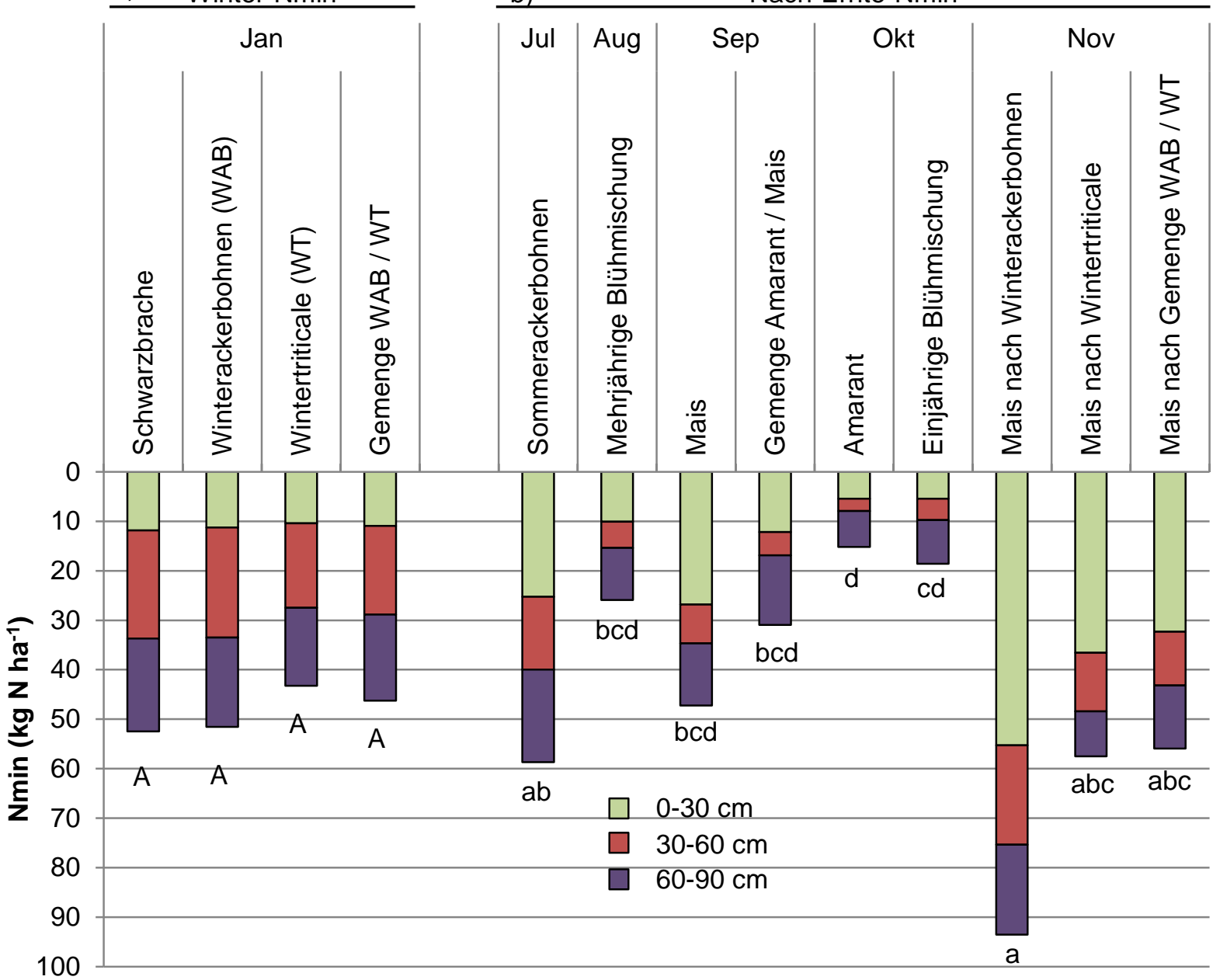

Nach-Ernte-Nmin

$\underline{b}$

Abbildung 18: Nmin-Mengen in drei Tiefenstufen im Versuchsjahr 2015 am Standort Schoningen unterteilt in die Probenahme im Winter (a) und nach der Ernte im Sommer bzw. Herbst (b). Varianten mit gleichen Großbuchstaben (Winter-Nmin) bzw. gleichen Kleinbuchstaben (Nach-Ernte-Nmin) sind nicht signifikant verschieden, $p<0,05$ (TukeyTest). Die statistischen Angaben beziehen sich auf die Höhe der Gesamtsäule.

Nach der Ernte der Feldfüchte des ersten Versuchsjahres zeigte sich ein mit dem Standort Reinshof vergleichbares Bild. Auch hier waren nach der Zweitfrucht Mais bei den Varianten des Zweikulturnutzungssystems und den Sommerackerbohnen die größten N-Mengen zu finden (Abbildung 18 b). Auffällig war wieder der Mais nach Winterackerbohnen mit $94 \mathrm{~kg} \mathrm{~N}$ $\mathrm{ha}^{-1}$. Der Mais als Sommerhauptfrucht sowie das Gemenge aus Amarant und Mais hinterlieBen zwar geringere N-Mengen mit $47 \mathrm{bzw} .31 \mathrm{~kg} \mathrm{~N}^{-1}$, signifikant unterschieden sie sich damit jedoch nicht von Mais als Zweitfrucht nach Wintertriticale und dem Gemenge aus Winterackerbohnen und Wintertriticale sowie den Sommerackerbohnen mit Werten zwischen 56 und $59 \mathrm{~kg} \mathrm{~N} \mathrm{ha}^{-1}$. Weniger aber wiederum nicht signifikant weniger als bei Mais und dem Gemenge aus Amarant und Mais war es bei den Blühmischungen und insbesondere beim Amarant. 


\section{Schoningen}

\section{Zweites Versuchsjahr}

Neben den Erstfrüchten gingen auch am Standort Schoningen in die Winter-Nmin-Untersuchung des zweiten Versuchsjahres die mehrjährigen Varianten ein (Abbildung 19 a). Dies waren auch die Varianten mit den geringsten N-Mengen im Boden. Jedoch unterschieden sich nur die mehrjährige Blühmischung und das Deutsche Weidelgras mit 19 bzw. $20 \mathrm{~kg} \mathrm{~N}^{\mathrm{N}} \mathrm{ha}^{-1}$ signifikant vom Wickroggen mit $39 \mathrm{~kg} \mathrm{~N}$ ha $^{-1}$. Die Erstfrüchte des Zweikulturnutzungssystems unterschieden sich mit Werten zwischen 27 und $39 \mathrm{~kg} \mathrm{~N} \mathrm{ha}^{-1}$ nicht signifikant voneinander und auch nicht von der Schwarzbrache mit $40 \mathrm{~kg} \mathrm{~N} \mathrm{ha}^{-1}$. Wie im ersten Versuchsjahr an diesem Standort beobachtet, wurden bei allen Erstfrüchten des Zweikulturnutzungssystems sowie der Schwarzbrache im Winter geringere N-Mengen gefunden, als mit $68 \mathrm{~kg} \mathrm{~N} \mathrm{ha}^{-1}$ zur Aussaat der Erstfrüchte im Oktober 2015 im Mittel über die Versuchsanlage im Boden vorlagen (Daten nicht dargestellt). Eine Veränderung der Nmin-Mengen in dieser Zeit zeigte sich vor allem in einer Abnahme im oberen $(0-30 \mathrm{~cm})$ und mittleren $(30-60 \mathrm{~cm})$ Bodenabschnitt bei allen Varianten, beim Wickroggen und der Schwarzbrache jedoch auch in einer Zunahme im unteren Bodenabschnitt $(60-90 \mathrm{~cm})$.

Den meisten mineralischen Stickstoff nach der Ernte hinterließ der nach Winterackerbohnen im Zweikulturnutzungssystem angebaute Mais mit $97 \mathrm{~kg} \mathrm{~N}^{-1}$ (Abbildung 19 b). Ebenfalls recht hoch, und nicht signifikant verschieden dazu, waren die Nmin-Werte von Mais nach Wintertriticale und dem Gemenge aus Winterackerbohnen und Wintertriticale. Besonders gering waren hier, wie am Standort Reinshof auch, die Nmin-Mengen beim Deutschen Weidelgras mit $8 \mathrm{~kg} \mathrm{~N} \mathrm{ha}^{-1}$. Signifikant unterschiedlich zu den anderen mehrjährigen Varianten, der mehrjährigen Blühmischung und der Durchwachsenen Silphie sowie den einjährigen Varianten Sommerackerbohnen, Amarant und einjährige Blühmischung mit Werten zwischen 15 und 23 $\mathrm{kg} \mathrm{N} \mathrm{ha}^{-1}$ war das Deutsche Weidelgras damit jedoch nicht. Auffällig bei den Nmin-Werten nach der Ernte waren, wie im Vorjahr und am Standort Reinshof ebenfalls beobachtet, die hohen N-Mengen im oberen Bodenabschnitt $(0-30 \mathrm{~cm})$ bei Mais als Zweitfrucht. Dies waren bis zu $72 \mathrm{~kg} \mathrm{~N} \mathrm{ha}^{-1}$ bei Mais nach Winterackerbohnen. 


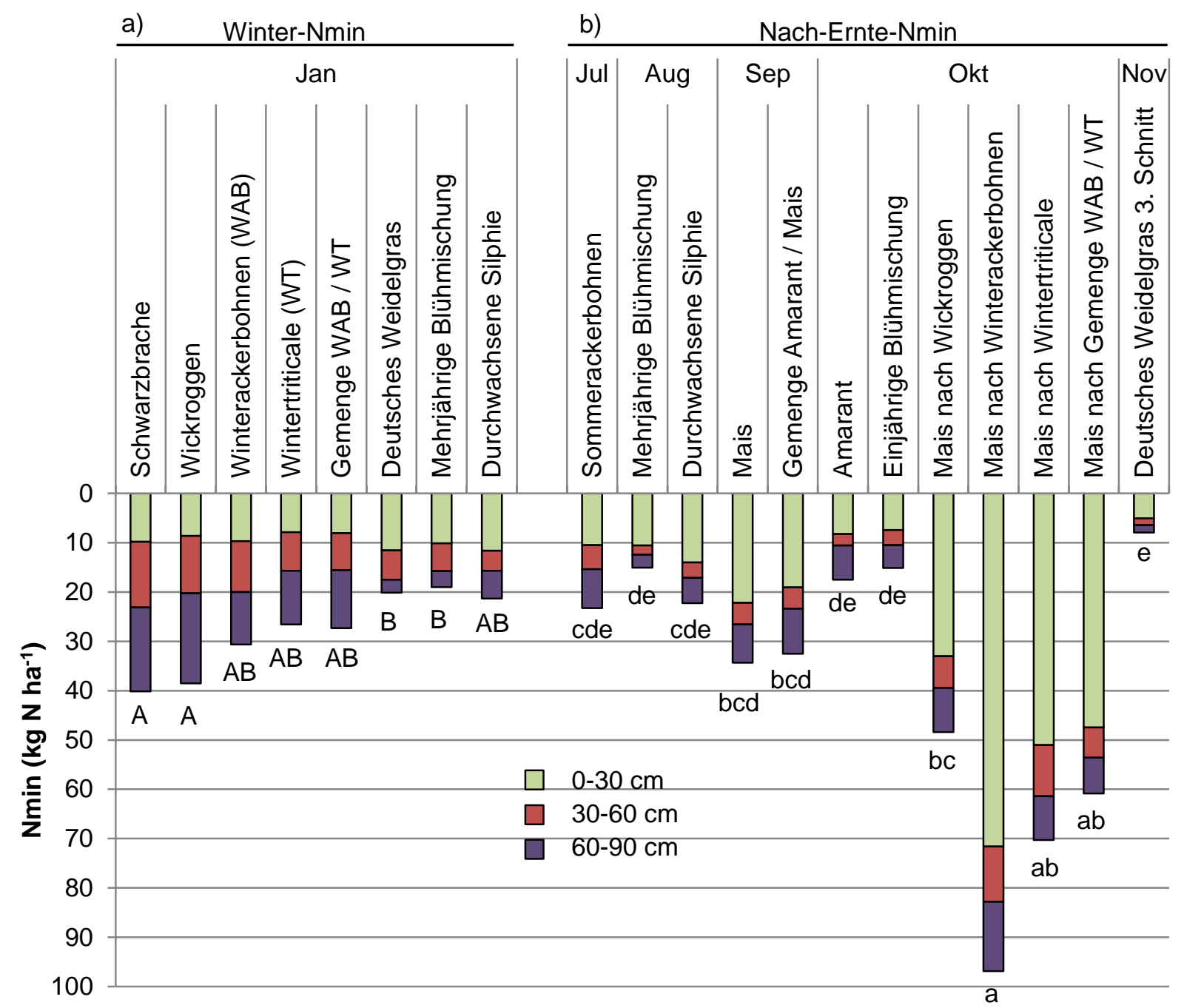

Abbildung 19: Nmin-Mengen in drei Tiefenstufen im Versuchsjahr 2016 am Standort Schoningen unterteilt in die Probenahme im Winter (a) und nach der Ernte im Sommer bzw. Herbst (b). Varianten mit gleichen Großbuchstaben (Winter-Nmin) bzw. gleichen Kleinbuchstaben (Nach-Ernte-Nmin) sind nicht signifikant verschieden, $p<0,05$ (TukeyTest). Die statistischen Angaben beziehen sich auf die Höhe der Gesamtsäule.

\subsubsection{Pflanzenverfügbares Bodenwasser}

Abbildung 20 und Abbildung 21 zeigen die zur Ernte der jeweiligen Varianten pflanzenverfügbaren Mengen an Wasser im Boden an den Standorten Reinshof und Schoningen. So ist die chronologische Abfolge der Ernten dargestellt, bei den Varianten des Zweikulturnutzungssystems sowohl die Ernte der Erst- als auch der Zweitfrucht und beim Deutschen Weidelgras die drei Schnitte pro Jahr. Zum Vergleich der theoretisch möglichen Menge an pflanzenverfügbarem Wasser ist auch die nutzbare Feldkapazität (nFK) gezeigt.

\section{Reinshof}

Am Standort Reinshof beanspruchten die Erstfrüchte des Zweikulturnutzungssystems im ersten Versuchsjahr den Bodenwasserhaushalt stark (Abbildung 20). So hinterließen der Wickroggen, die Winterackerbohnen, die Wintertriticale sowie das Gemenge aus Winterackerbohnen und Wintertriticale nach der Ernte nur etwa 8-10 mm Wasser in den oberen $30 \mathrm{~cm}$ und etwa 12 mm im Bodenabschnitt von 30-60 cm im Boden. Das war etwa ein Drittel des Wassers, 
das bei der Schwarzbrache zur gleichen Zeit in dieser Tiefe zur Verfügung stand und nur $15 \%$ nKF im oberen und $21 \%$ nFK im darunterliegenden Bodenabschnitt.

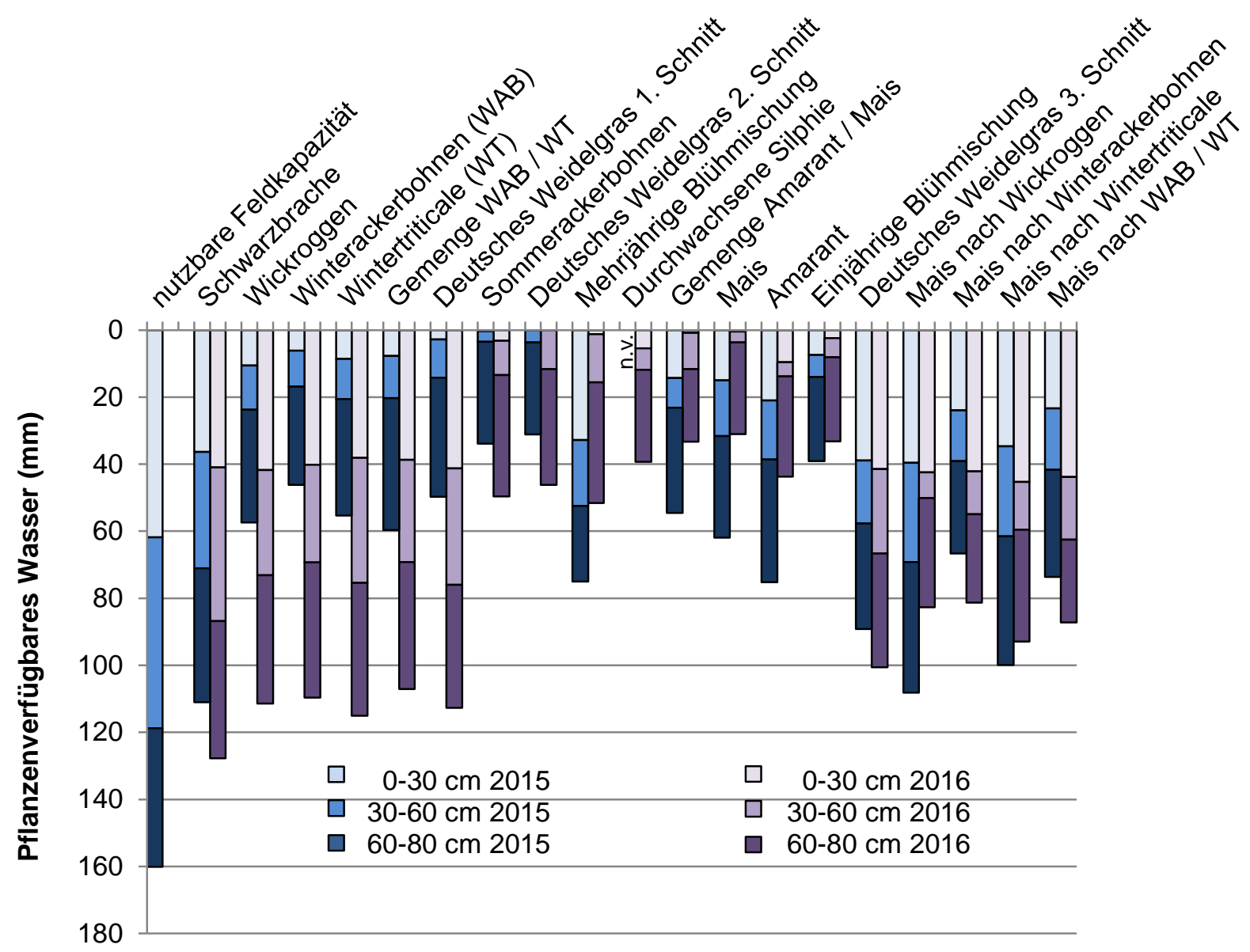

Abbildung 20: Menge an pflanzenverfügbarem Wasser nach der Ernte der Varianten in einer Bodentiefe von 80 cm unterteilt in drei Tiefenstufen in den Versuchsjahren 2015 und 2016 am Standort Reinshof, n.v. = Werte nicht verfügbar.

Im zweiten Versuchsjahr unterschied sich hier dagegen die Menge an pflanzenverfügbarem Wasser nach den Erstfrüchten des Zweikulturnutzungssystems kaum von der Schwarzbrache. Bei diesen Varianten standen etwa $40 \mathrm{~mm}$ Wasser (65 \% nKF) im oberen und weitere 30-35 $\mathrm{mm}(53-61 \% \mathrm{nFK})$ im Bodenabschnitt von 30-60 cm zur Verfügung. Das gleiche Bild zeigte sich für den ersten Schnitt des Deutschen Weidelgrases: Im ersten Versuchsjahr war der Boden vor allem im oberen Bodenabschnitt fast vollständig entleert, im zweiten Versuchsjahr hingegen ähnlich mit Wasser gefüllt wie bei der Schwarzbrache. Zum zweiten Schnitt des Deutschen Weidelgrases im Sommer beider Versuchsjahre waren die oberen $30 \mathrm{~cm}$ im Boden vollständig von pflanzenverfügbarem Wasser entleert und auch im darunterliegenden Bodenabschnitt $(30-60 \mathrm{~cm})$ war kaum noch Wasser verfügbar. Zum dritten Schnitt des Deutschen Weidelgrases im Herbst war in beiden Versuchsjahren insbesondere der obere Bodenabschnitt wieder mit Wasser aufgefüllt. Hier lagen etwa $40 \mathrm{~mm}$ pflanzenverfügbares Wasser vor.

Bei den in den Sommermonaten geernteten Varianten war bis auf ein paar Ausnahmen im ersten Versuchsjahr insgesamt wenig Wasser nach der Ernte verfügbar. Nach dem im Herbst geernteten Mais als Zweitfrucht im Zweikulturnutzungssystem war in beiden Versuchsjahren 
der Boden wieder mit Wasser aufgefüllt und etwa $40 \mathrm{~mm}$ im oberen Bodenabschnitt verfügbar. Nur bei Mais nach Winterackerbohnen und nach dem Gemenge aus Winterackerbohnen und Wintertriticale im ersten Versuchsjahr war es weniger. In keinem der beiden Versuchsjahre war der Boden an diesem Standort im Verlaufe der Vegetationszeit vollständig wassergesättigt und die nutzbare Feldkapazität erreicht. Einzig der untere Bodenabschnitt $(60-80 \mathrm{~cm})$ wurde weniger beansprucht als die oberen $(0-30$ und 30-60 cm). Hier waren nur bei den Ernten in den Sommermonaten etwas geringere Mengen als die nutzbare Feldkapazität erkennbar.

\section{Schoningen}

Am Standort Schoningen war vor allem im Unterboden weniger pflanzenverfügbares Wasser vorhanden als am Standort Reinshof. Im ersten Versuchsjahr war nach der Ernte der Erstfrüchte des Zweikulturnutzungssystems im Juni 2015 nahezu kein pflanzenverfügbares Wasser zu finden (Abbildung 21). Sowohl der Oberboden (0-30 cm) als auch der Unterboden (3060 bzw. $60-80 \mathrm{~cm}$ ) waren entleert. Bei der Schwarzbrache war es mit $30 \mathrm{~mm}(44 \% \mathrm{nFK})$ in 0$30 \mathrm{~cm}$ Bodentiefe und $36 \mathrm{~mm}$ (58\% nFK) in 30-60 cm doch deutlich mehr als nach Winterackerbohnen, Wintertriticale sowie dem Gemenge aus Winterackerbohnen und Wintertriticale. Bis zur Ernte der hier folgenden Zweitfrucht Mais war im mittleren Bodenabschnitt wieder etwas Wasser angekommen, aber mit etwa $20 \mathrm{~mm}$ bzw. $32 \%$ nKF war dies immer noch recht wenig. Im oberen Bodenabschnitt waren es etwa $30 \mathrm{~mm}$ pflanzenverfügbares Wasser. Die Varianten, die im ersten Versuchsjahr im Verlauf der Sommermonate geerntet wurden, hinterließen das Wasser überwiegend im oberen Bodenabschnitt.

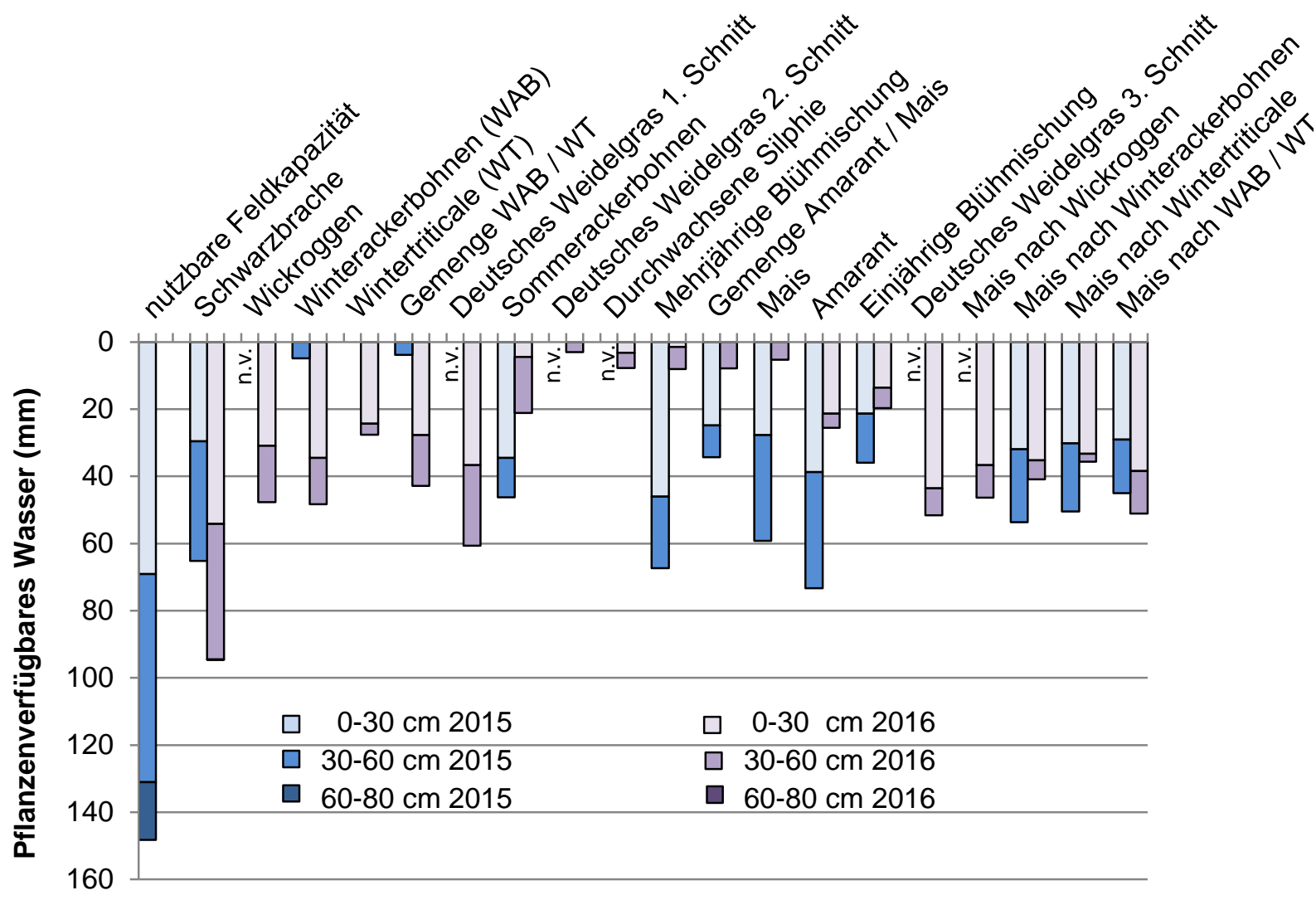

Abbildung 21: Menge an pflanzenverfügbarem Wasser nach der Ernte der Varianten in einer Bodentiefe von 80 cm unterteilt in drei Tiefenstufen in den Versuchsjahren 2015 und 2016 am Standort Schoningen, n.v. = Werte nicht verfügbar. 
Im zweiten Versuchsjahr war der Boden nach der Erstfruchternte nicht so ausgetrocknet wie im ersten Versuchsjahr. Mit etwa 25-35 mm bzw. 35-50 \% nFK in 0-30 cm Bodentiefe, 3-17 mm bzw. 5-27 \% nFK im Bodenabschnitt von 30-60 cm und überhaupt kein pflanzenverfügbares Wasser in 60-80 cm Bodentiefe waren die Wasserreserven aber dennoch gering. Nach Schwarzbrache über Winter stand etwa die doppelte Menge an pflanzenverfügbarem Wasser zur Verfügung als nach den Erstfrüchten des Zweikulturnutzungssystems. Auffällig waren weiterhin die sehr geringen Mengen an pflanzenverfügbarem Wasser im Boden nach der Ernte der mehrjährigen Arten Deutsches Weidelgras (2. Schnitt), Durchwachsene Silphie und mehrjährige Blühmischung sowie der Sommerhauptfrüchte Mais und Gemenge aus Amarant und Mais im zweiten Versuchsjahr. Hier waren die oberen $30 \mathrm{~cm}$ vollständig entleert und auch im darunterliegenden Bodenabschnitt war kaum noch Wasser verfügbar. Erst zur Ernte des Amarants und der einjährigen Blühmischung Anfang Oktober konnte wieder Wasser im oberen Bodenabschnitt gefunden werden. Bis zur Ernte von Mais als Zweitfrucht im Zweikulturnutzungssystem waren es immerhin wieder $35 \mathrm{~mm}$ (50\% nFK) pflanzenverfügbares Wasser in den oberen $30 \mathrm{~cm}$.

Bis auf die Schwarzbrache im zweiten Versuchsjahr konnte bei keiner der Probenahmen an diesem Standort pflanzenverfügbares Wasser im unteren Bodenabschnitt $(60-80 \mathrm{~cm})$ gefunden werden.

\subsection{Akzeptanz des Energiepflanzenanbaus in der Bevölkerung}

$88 \%$ der Befragten bewerten die Anstrengungen, fossile Energien durch erneuerbare (z.B. Windenergie, Photovoltaik, Biomasse) zu ersetzen, als gut bis sehr gut. Etwa $50 \%$ schätzen in diesem Zusammenhang die Nutzung von Biomasse in Form von Energiepflanzen für die Biogaserzeugung als gut bis sehr gut ein (Daten nicht dargestellt).

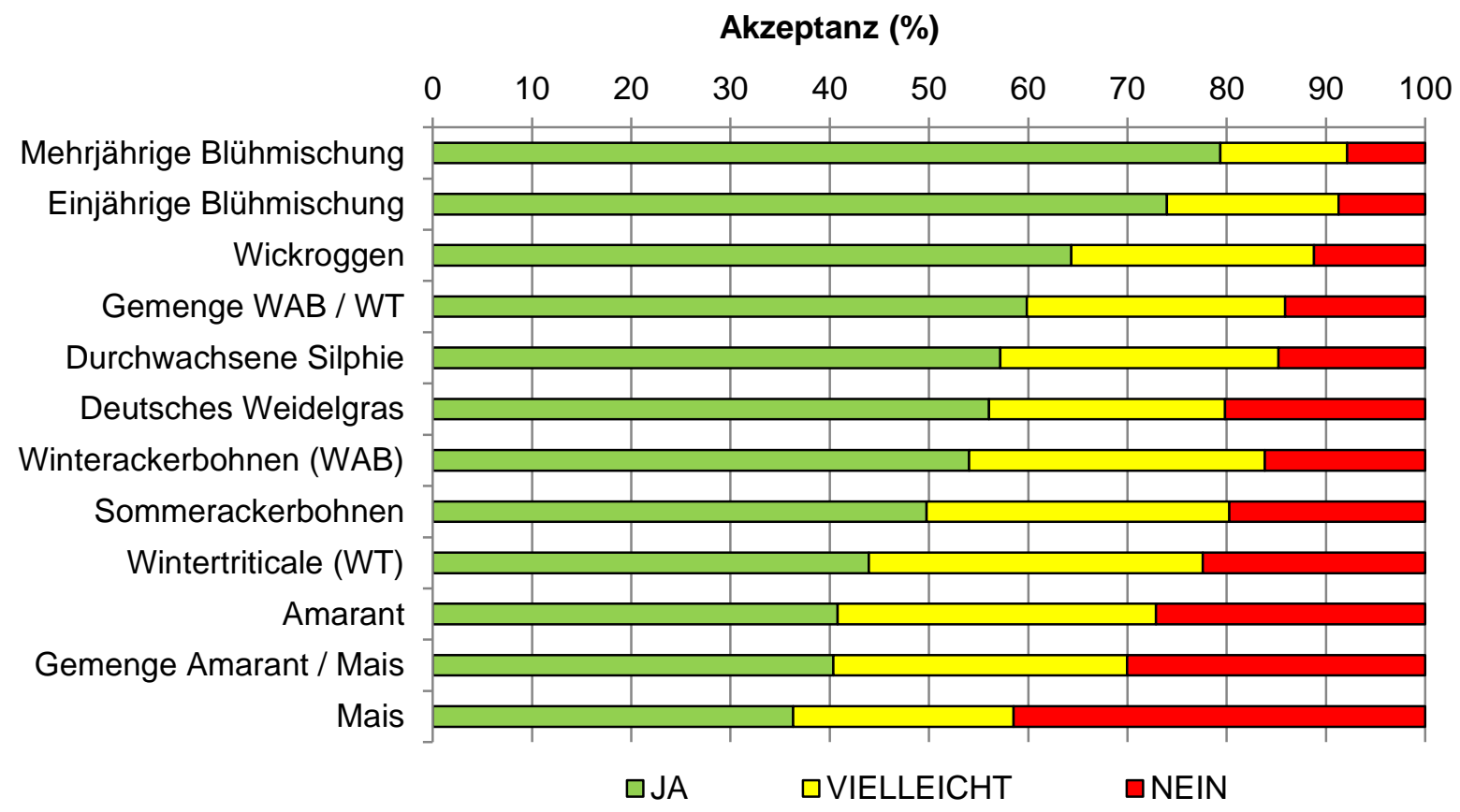

Abbildung 22: Gesellschaftliche Akzeptanz der untersuchten Feldfrüchte als Energiepflanzen für die Biogaserzeugung, $n=446$. 
Auf die Frage nach der Bewertung des Anbaus der von uns untersuchten Feldfrüchte zur Biogaserzeugung befürworteten der überwiegende Teil der Befragten die Blühmischungen für den Anbau (Abbildung 22). Neben dem optischen Anreiz, den etwa $57 \%$ bei der einjährigen und etwa $50 \%$ bei der mehrjährigen Blühmischung sehen, begründeten die Befragten ihre Bewertung dieser Varianten zu 34 bzw. 41 \% mit dem ökologischen Nutzen der davon ausgehe, etwa durch eine gesteigerte Artenvielfalt oder dem zusätzlichen Angebot an Nahrungspflanzen für Insekten (Tabelle 16). Probleme oder Nachteile wurden bei diesen Varianten kaum angemerkt.

Immerhin noch etwa zwei Drittel der Befragten können sich den Anbau von Wickroggen, dem Gemenge aus Winterackerbohnen und Wintertriticale, Durchwachsene Silphie und Deutsches Weidelgras als Energiepflanzen für die Biogaserzeugung vorstellen (Abbildung 22). Auch bei diesen Varianten trug, bis auf das Deutsche Weidelgras, mit bis zu $63 \%$ beim Wickroggen besonders das Aussehen zur positiven Bewertung bei (Tabelle 16). Aber auch viele ökologische Vorteile wurden diesen Varianten zugeschrieben. Beim Deutschen Weidelgras kamen diese Einschätzungen nicht so häufig vor. $27 \%$ empfinden es als ansehnliche Feldfrucht und nur $15 \%$ der Befragten meinen, es gäbe ökologische Vorteile beim Anbau von Deutschem Weidelgras. Dagegen denken etwa $20 \%$ der Befragten, das Deutsche Weidelgras führe zu ökologischen Nachteilen z.B. aufgrund der Mehrschnittigkeit oder dem begrenzten Lebensraum für Insekten. Immerhin weitere $10 \%$ der Befragten meinen, dass das Deutsche Weidelgras als traditionelle Feldfrucht in das Landschaftsbild gehöre.

Bei den Winter- und Sommerackerbohnen schlug sich die Verknüpfung mit positiven Eigenschaften bei den Befragten, also der ökologischen Vorteilhaftigkeit, mit etwa 39 bzw. $28 \%$ und ihrer Ansehnlichkeit mit etwa 42 bzw. 37 \% (Tabelle 16) nicht in einer größeren Akzeptanz für den Anbau als Energiepflanzen für die Biogaserzeugung nieder. Nur etwa die Hälfte der Befragten befürworten Winter- und Sommerackerbohnen im Energiepflanzenanbau (Abbildung 22).

Nur etwa $40 \%$ der Befragten stimmten dem Anbau von Wintertriticale, Amarant und dem Gemenge aus Amarant und Mais als Energiepflanzen für die Biogaserzeugung zu (Abbildung 22). Die Wintertriticale wurde zu etwa $22 \%$ der Befragten als unauffällig und wenig abgrenzbar zu anderen Getreidearten angesehen (Tabelle 16). Weitere $20 \%$ assoziieren mit ihr ökologische Nachteile. Auffällig bei der Bewertung des Amarants ist, dass etwa $28 \%$ der Befragten ihre Ablehnung auf das Nicht-Heimisch sein dieser Feldfrucht stützten. Zudem bringen $7 \%$ der Befragten Amarant mit Nahrungsmitteln in Verbindung und meinen, das sei die bessere Nutzung.

Die geringste Zustimmung für den Anbau erfuhr der Mais (Abbildung 22). Mit etwa $39 \%$ waren die bei den Befragten damit verbundenen ökologischen Nachteile, wie etwa Artenverarmung oder Bodenschäden, das häufigste Argument gegen den Maisanbau (Tabelle 16). Weitere $22 \%$ meinen, der Anbauumfang sei aktuell bereits zu hoch. Aber auch die hohe Bestandeshöhe des Maises störe etwa $12 \%$ der Befragten. 
Tabelle 16: Bewertung der untersuchten Feldfrüchte durch die Umfrageteilnehmer. Ergebnisse der Frequenzanalyse, Häufigkeitsangaben $(n=x)$ stellen das Auftreten der Kategorien im Fragebogenmaterial bei den einzelnen Feldfrüchten dar, Angaben in \%.

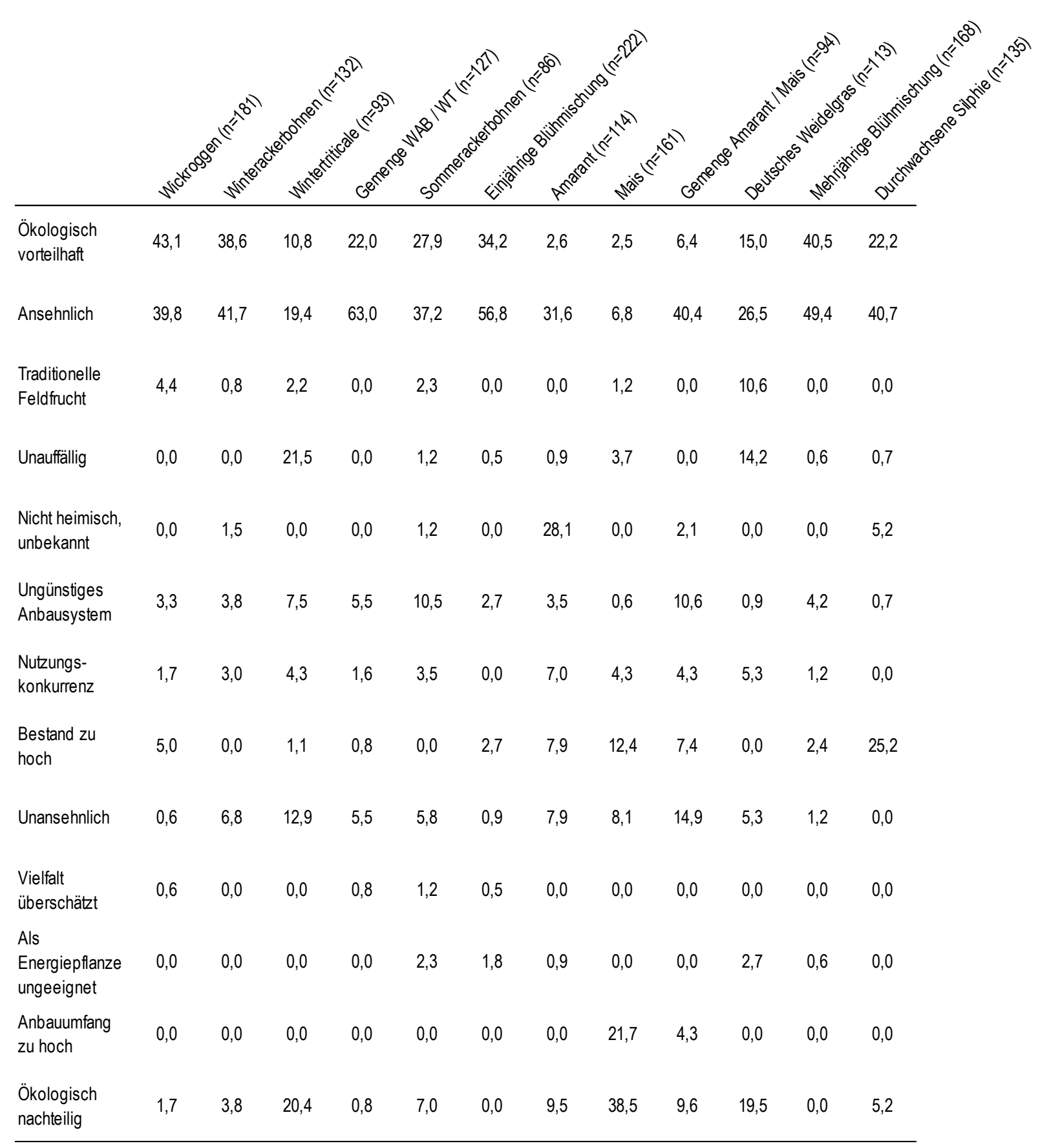




\section{5 Ökonomische Bewertung}

Die Höhe der erzielten Deckungsbeiträge unterschied sich in beiden Versuchsjahren sowohl stark zwischen den Varianten als auch zwischen den Standorten (Tabelle 17). Insgesamt rangierten die Deckungsbeiträge zwischen $-150 €$ ha $^{-1}$ bei der einjährigen Blühmischung im zweiten Versuchsjahr am Standort Schoningen und $1377 €$ ha $^{-1}$ bei Mais ebenfalls im zweiten Versuchsjahr am Standort Reinshof.

Im ersten Versuchsjahr erzielten am Standort Reinshof das Gemenge aus Amarant und Mais, das Deutschen Weidelgras und der Mais in Reinsaat vergleichbare Deckungsbeiträge zwischen 1220 und $1252 €$ ha-1 $^{-1}$ (Tabelle 17a). Mit deutlich geringeren Deckungsbeiträgen im Vergleich zu dieser Gruppe, schnitten die Varianten des Zweikulturnutzungssystems in diesem Versuchsjahr am Standort Reinshof ab (912-1076€ ha-1). Weit abgeschlagen bei der ökonomischen Bewertung waren die Sommerackerbohnen. Hier blieben nach Abzug der Kosten von den Leistungen nur $254 € \mathrm{ha}^{-1}$ übrig.

Am Standort Schoningen lagen die Deckungsbeiträge insgesamt auf einem niedrigeren $\mathrm{Ni}$ veau als am Standort Reinshof. Im ersten Versuchsjahr konnte hier der höchste Deckungsbeitrag bei der Mais-Reinsaat mit $945 €$ ha $^{-1}$ ermittelt werden (Tabelle 17b). Gleichauf war das Zweikulturnutzungssystem aus Wintertriticale gefolgt von Mais, wobei sich allerdings die Leistungs- und Kostenstrukturen bei diesen beiden Varianten enorm unterschieden. Die übrigen Varianten des Zweikulturnutzungssystems, Winterackerbohnen sowie das Gemenge aus Winterackerbohnen und Wintertriticale, jeweils gefolgt von Mais, konnten hier mit 598 bzw. $540 €$ ha $^{-1}$ nicht heranreichen. Wie am Standort Reinshof, erzielten auch am Standort Schoningen die Sommerackerbohnen mit $89 €$ ha $^{-1}$ die geringsten Deckungsbeiträge. Nicht viel stärker war hier jedoch auch die einjährige Blühmischung mit $165 €$ ha $^{-1}$.

Im zweiten Versuchsjahr konnten am Standort Reinshof die höchsten Deckungsbeiträge mit etwa $1380 €$ ha $^{-1}$ beim Anbau von Mais in Reinsaat ermittelt werden (Tabelle 17a). Auch die Varianten des Zweikulturnutzungssystems, das Gemenge aus Amarant und Mais sowie die Durchwachsene Silphie kamen mit einem Deckungsbeitrag von etwa 1200-1300 € ha-1 nah an den Mais in Reinsaat heran. Das Deutsche Weidelgras war im zweiten Versuchsjahr an diesem Standort zwar etwas schlechter als im ersten Versuchsjahr, ließ sich aber mit mehr als $1000 €$ ha $^{-1}$ eher in die Gruppe der hochleistenden Varianten einordnen. Deutlich geringer waren die Deckungsbeiträge der ein- und mehrjährigen Blühmischung sowie von Amarant. Das Schlusslicht mit $389 €$ ha $^{-1}$ bildeten auch im zweiten Versuchsjahr am Standort Reinshof die Sommerackerbohnen.

Am Standort Schoningen fiel im zweiten Versuchsjahr besonders das Deutsche Weidelgras auf, das mit $994 €$ ha $^{-1}$ einen deutlich höheren Deckungsbeitrag erzielte als der Mais in Reinsaat als zweitstärkste Variante mit $824 €$ ha-1 $^{-1}$ Tabelle 17b). Die Deckungsbeiträge der Varianten des Zweikulturnutzungssystems konnten im zweiten Versuchsjahr an diesem Standort bei weitem nicht mit der Mais-Reinsaat mithalten. Der Anteil des Maises als Zweitfrucht am Gesamtdeckungsbeitrag war hier bedingt durch die geringen Trockenmasseerträge dieser Feldfrucht gering. Die Durchwachsene Silphie ordnete sich mit einem Deckungsbeitrag von $500 € \mathrm{ha}^{-1} \mathrm{im}$ Mittelfeld ein. Wie auch am Standort Reinshof waren am Standort Schoningen die Deckungsbeiträge der Blühmischungen und von Amarant relativ gering. Bei der einjährigen 
Blühmischung konnten die variablen Kosten hier nicht durch die Leistungen gedeckt werden, sodass ein negativer Deckungsbeitrag entstand.

Tabelle 17: Kenngrößen der ökonomischen Bewertung (Leistung, Kosten, Deckungsbeitrag I) der untersuchten Varianten an den Standorten Reinshof (a) und Schoningen (b) in den Versuchsjahren 2015 und 2016.

\begin{tabular}{|c|c|c|c|c|c|c|}
\hline \multirow[t]{3}{*}{ a) } & \multicolumn{6}{|c|}{ Reinshof } \\
\hline & \multicolumn{3}{|c|}{2015} & \multicolumn{3}{|c|}{2016} \\
\hline & $\begin{array}{l}\text { Leistung } \\
\left(€ \text { ha }^{-1}\right)\end{array}$ & $\begin{array}{c}\text { Variable } \\
\text { Kosten } \\
\left(€ \mathrm{ha}^{-1}\right)\end{array}$ & $\begin{array}{c}\text { Deckungs- } \\
\text { beitrag I } \\
\left(€ \text { ha }^{-1}\right)\end{array}$ & $\begin{array}{l}\text { Leistung } \\
\left(€ \text { ha }^{-1}\right)\end{array}$ & $\begin{array}{l}\text { Variable } \\
\text { Kosten } \\
\left(€ \text { ha }^{-1}\right)\end{array}$ & $\begin{array}{c}\text { Deckungs- } \\
\text { beitrag I } \\
\left(€ \mathrm{ha}^{-1}\right)\end{array}$ \\
\hline Wickroggen + Mais & 2330 & 1254 & 1076 & 2641 & 1380 & 1261 \\
\hline Winterackerbohnen (WAB) + Mais & 2213 & 1310 & 903 & 2668 & 1438 & 1230 \\
\hline Wintertriticale (WT) + Mais & 2330 & 1302 & 1028 & 2680 & 1378 & 1303 \\
\hline Gemenge WAB / WT + Mais & 2261 & 1348 & 912 & 2562 & 1456 & 1106 \\
\hline Sommerackerbohnen & 930 & 676 & 254 & 1089 & 700 & 389 \\
\hline Einjährige Blühmischung & 1093 & 491 & 602 & 1153 & 497 & 656 \\
\hline Amarant & 1194 & 469 & 726 & 1228 & 536 & 692 \\
\hline Mais & 1914 & 694 & 1220 & 2142 & 765 & 1377 \\
\hline Gemenge Amarant / Mais & 2034 & 783 & 1252 & 2092 & 864 & 1228 \\
\hline Deutsches Weidelgras & 1730 & 487 & 1243 & 1566 & 543 & 1023 \\
\hline Mehrjährige Blühmischung & 822 & 355 & 466 & 985 & 432 & 552 \\
\hline Durchwachsenen Silphie & n.v. & n.v. & n.v. & 1745 & 528 & 1217 \\
\hline
\end{tabular}

\begin{tabular}{|c|c|c|c|c|c|c|}
\hline \multirow[t]{3}{*}{ b) } & \multicolumn{6}{|c|}{ Schoningen } \\
\hline & \multicolumn{3}{|c|}{2015} & \multicolumn{3}{|c|}{2016} \\
\hline & $\begin{array}{l}\text { Leistung } \\
\left(€ \mathrm{ha}^{-1}\right)\end{array}$ & $\begin{array}{c}\text { Variable } \\
\text { Kosten } \\
\left(€ \text { ha-1) }^{-1}\right.\end{array}$ & $\begin{array}{c}\text { Deckungs- } \\
\text { beitrag I } \\
\left(€ \text { ha }^{-1}\right)\end{array}$ & $\begin{array}{l}\text { Leistung } \\
\left(€ \text { ha-1 }^{-1}\right)\end{array}$ & $\begin{array}{c}\text { Variable } \\
\text { Kosten } \\
\left(€ \mathrm{ha}^{-1}\right)\end{array}$ & $\begin{array}{c}\text { Deckungs- } \\
\text { beitrag I } \\
\left(€ \text { ha-1) }^{-1}\right.\end{array}$ \\
\hline Wickroggen + Mais & n.v. & n.v. & n.v. & 1643 & 1339 & 303 \\
\hline Winterackerbohnen (WAB) + Mais & 1917 & 1319 & 598 & 1822 & 1442 & 380 \\
\hline Wintertriticale (WT) + Mais & 2157 & 1213 & 944 & 1924 & 1423 & 501 \\
\hline Gemenge WAB / WT + Mais & 1896 & 1355 & 540 & 1777 & 1458 & 319 \\
\hline Sommerackerbohnen & 727 & 638 & 89 & 1018 & 658 & 359 \\
\hline Einjährige Blühmischung & 641 & 475 & 165 & 324 & 472 & -148 \\
\hline Amarant & 929 & 456 & 474 & 828 & 562 & 266 \\
\hline Mais & 1645 & 699 & 945 & 1604 & 780 & 824 \\
\hline Gemenge Amarant / Mais & 1544 & 778 & 766 & 1549 & 869 & 680 \\
\hline Deutsches Weidelgras & n.v. & n.v. & n.v. & 1527 & 534 & 994 \\
\hline Mehrjährige Blühmischung & 613 & 339 & 275 & 566 & 439 & 128 \\
\hline Durchwachsenen Silphie & n.v. & n.v. & n.v. & 999 & 492 & 507 \\
\hline
\end{tabular}




\subsection{Zusammenhänge zwischen ausgewählten Parametern der Energiepflanzenvarianten}

Einen sehr engen linearen Zusammenhang gab es zwischen dem Trockenmasseertrag und dem Methanflächenertrag ( $r=0,981$, Tabelle 18). Ebenfalls eng korreliert mit dem Trockenmasseertrag war mit einem Korrelationskoeffizienten von 0,738 die im Spross gebundene NMenge. Auch der Methanflächenertrag war recht eng positiv korreliert mit der N-Menge im Spross $(r=0,717)$. Einen mittleren negativen, aber hoch signifikanten Zusammenhang hatte der Trockenmasseertrag sowie der Methanflächenertrag mit dem Gehalt an Cobalt in der Spross-Trockenmasse ( $r=-0,521$ bzw. $r=-0,529)$. Insgesamt gab es überwiegend vergleichbare Korrelationen des Trockenmasseertrags und des Methanflächenertrags mit den anderen Parametern.

Ein mit $r=0,347$ nur geringer, aber signifikanter Zusammenhang fand sich zwischen der oberund unterirdischen Biomasse. Die N-Menge in der ober- und unterirdischen Biomasse hingegen hatte einen deutlicheren Zusammenhang mit $r=0,607$. Enger noch als der Trockenmasseertrag und die N-Menge im Spross war die Wurzel-Trockenmasse mit der in den Wurzeln gespeicherten N-Menge korreliert $(r=0,910)$.

Die Gehalte der beiden untersuchten Spurenelemente Cobalt und Nickel korrelierten mit einem Korrelationskoeffizienten von 0,530 mäßig aber hoch signifikant miteinander. Zwischen der Nmin-Menge im Boden nach der Ernte und dem Trockenmasseertrag gab es einen geringen, zwischen der Nmin-Menge und der N-Menge im Spross jedoch einen zwar nicht sehr engen, aber dennoch mit $r=0,622$ nennenswerten Zusammenhang.

Die Daten zum Bodenwasser sowie die Kenngrößen zur sozialen und ökonomischen Bewertung der Varianten gingen nicht in die Korrelationsanalyse ein. 
Tabelle 18: Korrelationskoeffizienten ( $r$ ) ausgewählter Parameter für die vereinigten Werte aus den Versuchsjahren 2015 und 2016 sowie den Standorten Reinshof und Schoningen, $n=174$, Korrelation nach Pearson, Korrelationskoeffizienten signifikant bei $p \leq 0,05$.

\begin{tabular}{|c|c|c|c|c|c|c|c|c|}
\hline & $\begin{array}{l}\text { Trockenmasse- } \\
\text { ertrag }\end{array}$ & $\begin{array}{c}\text { Trockensubstanz- } \\
\text { gehalt }\end{array}$ & $\begin{array}{l}\text { N-Menge } \\
\text { Spross }\end{array}$ & $\begin{array}{l}\text { Methanflächen- } \\
\text { ertrag }\end{array}$ & $\begin{array}{c}\text { Wurzel- } \\
\text { Trockenmasse }\end{array}$ & $\begin{array}{l}\text { N-Menge } \\
\text { Wurzeln }\end{array}$ & $\begin{array}{l}\text { Spuren- } \\
\text { elementgehalt } \\
\text { - Cobalt }\end{array}$ & $\begin{array}{c}\text { Spuren- } \\
\text { elementgehalt } \\
\text { - Nickel }\end{array}$ \\
\hline $\begin{array}{l}\text { Trockensubstanz- } \\
\text { gehalt }\end{array}$ & $\begin{array}{c}r=0,084 \\
p=0,2556\end{array}$ & & & & & & & \\
\hline $\begin{array}{l}\text { N-Menge } \\
\text { Spross }\end{array}$ & $\begin{array}{c}r=0,738 \\
p<2,2 e-16\end{array}$ & $\begin{array}{c}r=-0,381 \\
p=1,797 e-07\end{array}$ & & & & & & \\
\hline $\begin{array}{l}\text { Methanflächen- } \\
\text { ertrag }\end{array}$ & $\begin{array}{c}r=0,981 \\
p<2,2 e-16\end{array}$ & $\begin{array}{l}r=-0,169 \\
p=0,0248\end{array}$ & $\begin{array}{c}r=0,717 \\
p<2,2 e-16\end{array}$ & & & & & \\
\hline $\begin{array}{l}\text { Wurzel- } \\
\text { Trockenmasse }\end{array}$ & $\begin{array}{c}r=0,347 \\
p=2,435 e-06\end{array}$ & $\begin{array}{c}r=-0,455 \\
p=2,58 e-10\end{array}$ & $\begin{array}{c}r=0,556 \\
p=1,083 e-15\end{array}$ & $\begin{array}{c}r=0,290 \\
p=9,75 e-05\end{array}$ & & & & \\
\hline $\begin{array}{l}\text { N-Menge } \\
\text { Wurzeln }\end{array}$ & $\begin{array}{c}r=0,237 \\
p=0,0015\end{array}$ & $\begin{array}{c}r=-0,544 \\
p=6,103 e-15\end{array}$ & $\begin{array}{c}r=0,606 \\
p<2,2 e-16\end{array}$ & $\begin{array}{c}r=0,179 \\
p=0,0176\end{array}$ & $\begin{array}{c}r=0,910 \\
p<2,2 e-16\end{array}$ & & & \\
\hline $\begin{array}{l}\text { Spurenelement- } \\
\text { gehalt - Cobalt }\end{array}$ & $\begin{array}{c}r=-0,521 \\
p=1,284 e-13\end{array}$ & $\begin{array}{c}r=-0,487 \\
p=6,94 e-12\end{array}$ & $\begin{array}{l}r=-0,004 \\
p=0,9567\end{array}$ & $\begin{array}{c}r=-0,529 \\
p=4,684 e-14\end{array}$ & $\begin{array}{c}r=0,176 \\
p=0,0196\end{array}$ & $\begin{array}{c}r=0,392 \\
p<0,0001\end{array}$ & & \\
\hline $\begin{array}{l}\text { Spurenelement- } \\
\text { gehalt - Nickel }\end{array}$ & $\begin{array}{l}r=-0,179 \\
p=0,0174\end{array}$ & $\begin{array}{c}r=-0,534 \\
p=2,34 e-14\end{array}$ & $\begin{array}{c}r=0,193 \\
p=0,0102\end{array}$ & $\begin{array}{l}r=-0,235 \\
p=0,0016\end{array}$ & $\begin{array}{c}r=0,581 \\
p<2,2 e-16\end{array}$ & $\begin{array}{c}r=0,628 \\
p<2,2 e-16\end{array}$ & $\begin{array}{c}r=0,530 \\
p=4,09 e-14\end{array}$ & \\
\hline Nach-Ernte-Nmin & $\begin{array}{c}r=0,351 \\
p=1,787 e-06\end{array}$ & $\begin{array}{c}r=-0,290 \\
p=9,6 e-05\end{array}$ & $\begin{array}{c}r=0,622 \\
p<2,2 e-16\end{array}$ & $\begin{array}{c}r=0,376 \\
p=2,693 e-07\end{array}$ & $\begin{array}{c}r=0,237 \\
p=0,0016\end{array}$ & $\begin{array}{c}r=0,340 \\
p=3,383 e-06\end{array}$ & $\begin{array}{c}r=0,169 \\
p=0,0248\end{array}$ & $\begin{array}{c}r=0,064 \\
p=0,3967\end{array}$ \\
\hline
\end{tabular}




\subsection{Index der relativen Anbauwürdigkeit (IrA)}

\subsubsection{Teilindizes des Index IrA}

\section{Reinshof}

\section{Teilindex Trockenmasseertrag}

Am Standort Reinshof hatten in beiden Versuchsjahren die Varianten des Zweikulturnutzungssystems aber auch der Mais und das Gemenge aus Amarant und Mais hohe positive Werte für den Teilindex Trockenmasseertrag (Tabelle 19). Besonders im zweiten Versuchsjahr fielen hier die Varianten des Zweikulturnutzungssystems mit Werten zwischen 0,330 und 0,418 im Vergleich zu den anderen Varianten aber auch gegenüber Mais als Sommerhauptfrucht und dem Gemenge aus Amarant und Mais mit Indexwerten von 0,114 bzw. 0,082 auf.

Den niedrigsten Wert für den Teilindex Trockenmasseertrag hatten sowohl im ersten als auch im zweiten Versuchsjahr die Sommerackerbohnen mit -0,501 bzw. -0,476. Damit war dies die Variante mit dem größten Abstand der Trockenmasseerträge zum Mittel aller Varianten. Auch die Blühmischungen und der Amarant waren in beiden Versuchsjahren im deutlich negativen Wertebereich. Das Deutsche Weidelgras hatte im ersten Versuchsjahr einen knapp positiven Betrag beim Teilindex Trockenmasseertrag, im zweiten Versuchsjahr jedoch einen negativen.

\section{Teilindex TS-Gehalt}

Der bei den Zweikulturnutzungssystemen aus den Trockensubstanzgehalten der Erst- und Zweitfrucht berechnete Teilindex TS-Gehalt war bei diesen Varianten im ersten Versuchsjahr durchweg negativ (Tabelle 19). Im zweiten Versuchsjahr dagegen war dies nur bei Winterackerbohnen gefolgt von Mais der Fall. Die übrigen Varianten innerhalb der Zweikulturnutzungssysteme hatten positive Indexwerte. Die höchsten positiven Werte für den Teilindex TSGehalt zeigten in beiden Versuchsjahren der Mais und das Gemenge aus Amarant und Mais. Deutlich negativ waren die Indexwerte bei den Sommerackerbohnen, der einjährigen Blühmischung und dem Amarant aber auch bei der Durchwachsenen Silphie.

\section{Teilindex Methan}

Im ersten Versuchsjahr hatten, abgesehen von den Winterackerbohnen gefolgt von Mais, die Varianten des Zweikulturnutzungssystems und der Mais als Sommerhauptfrucht sowie das Gemenge aus Amarant und Mais etwa vergleichbare Werte für den Teilindex Methan im Bereich von 0,278 bis 0,386 (Tabelle 19). Im zweiten Versuchsjahr dagegen zeigten, ähnlich dem Teilindex TM-Ertrag, die Varianten des Zweikulturnutzungssystems höhere Werte für den Teilindex Methan als die maisbetonten Sommerhauptfrüchte. Die schwächsten Varianten waren in beiden Versuchsjahren analog zum Teilindex Trockenmasseertrag die Sommerackerbohnen, die Blühmischungen und der Amarant mit Werten zwischen -0,404 und -0,551.

Wie auch beim Teilindex Trockenmasseertrag hatte das Deutsches Weidelgras im ersten Versuchsjahr einen positiven und im zweiten Versuchsjahr einen negativen Wert für den Teilindex Methan. 
Tabelle 19: Teilindizes des Index IrA für die erhobenen Parameter Trockenmasseertrag (TM-Ertrag), Trockensubstanzgehalt (TS-Gehalt), Methanflächenertrag, Nmin, Wurzel-Trockenmasse, Bodenwasser, Spurenelemente (Co, $\mathrm{Ni}$ ) und ökonomische Aspekte (Deckungsbeitrag) in den Versuchsjahren 2015 und 2016 am Standort Reinshof.

\begin{tabular}{|c|c|c|c|c|c|c|c|c|}
\hline & $\begin{array}{l}\text { Teilindex } \\
\text { TM-Ertrag }\end{array}$ & $\begin{array}{l}\text { Teilindex } \\
\text { TS-Gehalt }\end{array}$ & $\begin{array}{l}\text { Teilindex } \\
\text { Methan }\end{array}$ & $\begin{array}{l}\text { Teilindex } \\
\text { Nmin }\end{array}$ & $\begin{array}{c}\text { Teilindex } \\
\text { Wurzel- } \\
\text { TM }\end{array}$ & $\begin{array}{l}\text { Teilindex } \\
\text { Boden- } \\
\text { wasser }\end{array}$ & $\begin{array}{l}\text { Teilindex } \\
\text { Spuren- } \\
\text { elemente }\end{array}$ & $\begin{array}{c}\text { Teilindex } \\
\text { Ökon. As- } \\
\text { pekte }\end{array}$ \\
\hline \multicolumn{9}{|c|}{2015} \\
\hline WIR + Mais & 0,328 & $-0,019$ & 0,380 & 0,260 & 0,221 & 0,063 & $-0,386$ & 0,223 \\
\hline WAB + Mais & 0,175 & $-0,249$ & 0,132 & $-0,824$ & 1,004 & $-0,289$ & 0,864 & 0,026 \\
\hline WT + Mais & 0,315 & $-0,038$ & 0,365 & 0,198 & 0,064 & $-0,007$ & $-0,383$ & 0,168 \\
\hline WAB / WT + Mais & 0,273 & $-0,132$ & 0,278 & 0,053 & 0,833 & $-0,170$ & 0,173 & 0,037 \\
\hline$S A B$ & $-0,501$ & $-0,305$ & $-0,550$ & $-0,391$ & 0,569 & $-0,035$ & 1,347 & $-0,712$ \\
\hline Einj. Blühm. & $-0,281$ & $-0,020$ & $-0,432$ & 0,212 & $-0,810$ & $-0,149$ & $-0,514$ & $-0,316$ \\
\hline Amarant & $-0,335$ & $-0,179$ & $-0,404$ & 0,201 & $-0,879$ & 0,106 & $-0,345$ & $-0,176$ \\
\hline Mais & 0,171 & 0,524 & 0,319 & $-0,166$ & $-0,491$ & 0,013 & $-0,577$ & 0,386 \\
\hline Amarant / Mais & 0,237 & 0,463 & 0,386 & $-0,007$ & $-0,581$ & $-0,039$ & $-0,536$ & 0,422 \\
\hline Deut. Weidelgras & 0,097 & $-0,060$ & 0,077 & 0,397 & 0,742 & 0,402 & 0,500 & 0,413 \\
\hline Mehrj. Blühm. & $-0,479$ & 0,015 & $-0,551$ & 0,068 & $-0,672$ & 0,105 & $-0,144$ & $-0,470$ \\
\hline Durchw. Silphie & n.v. & n.v. & n.v. & n.v. & n.v. & n.v. & n.v. & n.v. \\
\hline \multicolumn{9}{|c|}{2016} \\
\hline WIR + Mais & 0,409 & 0,157 & 0,500 & $-0,256$ & $-0,005$ & 0,141 & $-0,498$ & 0,258 \\
\hline WAB + Mais & 0,330 & $-0,015$ & 0,349 & $-0,659$ & 0,922 & 0,122 & 0,842 & 0,227 \\
\hline WT + Mais & 0,418 & 0,136 & 0,508 & $-0,152$ & 0,012 & 0,242 & $-0,472$ & 0,299 \\
\hline WAB / WT + Mais & 0,363 & 0,080 & 0,423 & $-0,177$ & 0,603 & 0,163 & 0,071 & 0,103 \\
\hline$S A B$ & $-0,476$ & $-0,335$ & $-0,521$ & $-0,231$ & 0,439 & 0,010 & 1,260 & $-0,612$ \\
\hline Einj. Blühm. & $-0,367$ & $-0,223$ & $-0,494$ & 0,170 & $-0,050$ & $-0,219$ & $-0,390$ & $-0,346$ \\
\hline Amarant & $-0,412$ & $-0,163$ & $-0,466$ & 0,199 & $-0,866$ & $-0,130$ & $-0,266$ & $-0,310$ \\
\hline Mais & 0,114 & 0,264 & 0,269 & $-0,477$ & $-0,838$ & $-0,238$ & $-0,589$ & 0,373 \\
\hline Amarant / Mais & 0,082 & 0,209 & 0,219 & $-0,450$ & $-0,757$ & $-0,218$ & $-0,551$ & 0,225 \\
\hline Deut. Weidelgras & $-0,114$ & $-0,065$ & $-0,120$ & 0,800 & 1,400 & 0,105 & 0,538 & 0,020 \\
\hline Mehrj. Blühm. & $-0,384$ & 0,166 & $-0,483$ & 0,605 & $-0,729$ & 0,105 & $-0,063$ & $-0,449$ \\
\hline Durchw. Silphie & 0,037 & $-0,210$ & $-0,184$ & 0,626 & $-0,132$ & $-0,081$ & 0,118 & 0,213 \\
\hline
\end{tabular}

$\mathrm{WIR}=$ Wickroggen, $\mathrm{WAB}=$ Winterackerbohnen, $\mathrm{WT}=$ Wintertriticale, $\mathrm{SAB}=$ Sommerackerbohnen, n.v. $=$ Werte nicht verfügbar.

\section{Teilindex Nmin}

Den höchsten Wert für den aus im Winter sowie nach der Ernte im Boden hinterlassenen NminMengen berechneten Teilindex Nmin hatte in beiden Versuchsjahren das Deutsche Weidelgras mit 0,397 bzw. 0,800 (Tabelle 19). Indexwerte im positiven Wertebereich hatten auch die Blühmischungen, der Amarant und die Durchwachsene Silphie sowie zumindest im ersten Versuchsjahr die Varianten des Zweikulturnutzungssystems, ausgenommen Winterackerbohnen gefolgt von Mais. Diese Variante hatte in beiden Versuchsjahren den größten negativen Wert für den Teilindex Nmin mit $-0,824$ bzw. -0,659. Negative Indexwerte hatten in beiden Versuchsjahren auch die Sommerackerbohnen, der Mais und das Gemenge aus Amarant und Mais.

\section{Teilindex Wurzel-Trockenmasse}

Beim Teilindex Wurzeltrockenmasse zeigten sich am Standort Reinshof vor allem im zweiten Versuchsjahr große Unterschiede zwischen den Varianten. Hohe Indexwerte hatten in beiden Versuchsjahren die Winterackerbohnen mit 1,004 bzw. 0,922 (Tabelle 19). An den Indexwert 
des Deutschen Weidelgrases im zweiten Versuchsjahr mit 1,400 kam jedoch keine der anderen Varianten heran. Geringe Werte für diesen Teilindex hatten die einjährige Blühmischung im ersten, der Mais, das Gemenge aus Amarant und Mais sowie die mehrjährige Blühmischung in beiden Versuchsjahren; die geringsten Indexwerte erzielte der Amarant mit -0,879 bzw. $-0,866$.

\section{Teilindex Bodenwasser}

Der Teilindex Bodenwasser, der das pflanzenverfügbare Wasser zu Vegetationsbeginn und nach der Ernte berücksichtigt, war der Teilindex mit der geringsten Übereinstimmung der Werte zwischen den Versuchsjahren (Tabelle 19). Während im ersten Versuchsjahr die Varianten des Zweikulturnutzungssystems bis auf Wickroggen gefolgt von Mais sowie die Sommerackerbohnen negative Indexwerte hatten, waren sie bei diesen Varianten im zweiten Versuchsjahr positiv. Bei Amarant und Mais war es genau umgekehrt.

Den höchsten positiven Wert beim Teilindex Bodenwasser hatte im ersten Versuchsjahr das Deutsche Weidelgras mit 0,402 und im zweiten Versuchsjahr das Zweikulturnutzungssystem mit Wintertriticale als Erst- und Mais als Zweitfrucht mit 0,242.

\section{Teilindex Spurenelemente}

Die Berechnung des Teilindex Spurenelemente aus der Spurenelementabfuhr (Cobalt und Nickel) ergab deutliche Unterschiede zwischen den Varianten. Den mit Abstand höchsten Wert beim Teilindex Spurenelemente wiesen in beiden Versuchsjahren mit 1,347 bzw. 1,260 die Sommerackerbohnen auf (Tabelle 19). Im Vergleich mit den überwiegend geringen Werten bei diesem Teilindex hatten aber auch die Winterackerbohnen gefolgt von Mais sowie das Deutsche Weidelgras mit 0,864 bzw. 0,500 im ersten und 0,842 bzw. 0,538 im zweiten Versuchsjahr relativ hohe Indexwerte. Die niedrigsten Indexwerte im Bereich von -0,589 bis -0,536 wurden in beiden Versuchsjahren bei Mais und dem Gemenge aus Amarant und Mais ermittelt.

\section{Teilindex Ökonomische Aspekte}

Im ersten Versuchsjahr waren das Gemenge aus Amarant und Mais sowie das Deutsche Weidelgras gleichauf beim Teilindex Ökonomische Aspekte und zeigten 0,422 bzw. 0,413 die höchsten Werte (Tabelle 19). Dann erst folgte der Mais in Reinsaat mit 0,386. Die Varianten des Zweikulturnutzungssystems blieben mit Werten zwischen 0,026 und 0,223 deutlich dahinter zurück. Im zweiten Versuchsjahr zeigte der Mais in Reinsaat mit 0,372 den höchsten Indexwert aber der Abstand zu den Varianten des Zweikulturnutzungssystems, Wickroggen und Wintertriticale jeweils gefolgt von Mais mit 0,258 bzw. 0,299 war hier nicht so groß wie im ersten Versuchsjahr. Indexwerte im deutlich negativen Wertebereich hatten in beiden Versuchsjahren die Sommerackerbohnen, die Blühmischungen und der Amarant.

\section{Schoningen}

\section{Teilindex Trockenmasseertrag}

Am Standort Schoningen hatte in beiden Versuchsjahren das Zweikulturnutzungssystem aus Wintertriticale als Erst- und Mais als Zweitfrucht mit 0,625 bzw. 0,384 den höchsten Wert beim Teilindex Trockenmasseertrag (Tabelle 20). Recht hohe Indexwerte zeigten hier im ersten Versuchsjahr auch die übrigen Varianten des Zweikulturnutzungssystems. Mit 0,342 bzw. 0,376 
Tabelle 20: Teilindizes des Index IrA für die erhobenen Parameter Trockenmasseertrag (TM-Ertrag), Trockensubstanzgehalt (TS-Gehalt), Methanflächenertrag, Nmin, Wurzel-Trockenmasse, Bodenwasser, Spurenelemente (Co, $\mathrm{Ni}$ ) und ökonomische Aspekte (Deckungsbeitrag) in den Versuchsjahren 2015 und 2016 am Standort Schoningen.

\begin{tabular}{|c|c|c|c|c|c|c|c|c|}
\hline & $\begin{array}{l}\text { Teilindex } \\
\text { TM-Ertrag }\end{array}$ & $\begin{array}{l}\text { Teilindex } \\
\text { TS-Gehalt }\end{array}$ & $\begin{array}{l}\text { Teilindex } \\
\text { Methan }\end{array}$ & $\begin{array}{l}\text { Teilindex } \\
\text { Nmin }\end{array}$ & $\begin{array}{c}\text { Teilindex } \\
\text { Wurzel-TM }\end{array}$ & $\begin{array}{l}\text { Teilindex } \\
\text { Boden- } \\
\text { wasser }\end{array}$ & $\begin{array}{l}\text { Teilindex } \\
\text { Spuren- } \\
\text { elemente }\end{array}$ & $\begin{array}{c}\text { Teilindex } \\
\text { Ökon. As- } \\
\text { pekte }\end{array}$ \\
\hline \multicolumn{9}{|c|}{2015} \\
\hline WIR + Mais & n.v. & n.v. & n.v. & n.v. & n.v. & n.v. & n.v. & n.v. \\
\hline WAB + Mais & 0,342 & $-0,203$ & 0,291 & $-0,670$ & 0,809 & $-0,432$ & 0,409 & 0,122 \\
\hline WT + Mais & 0,625 & 0,113 & 0,682 & $-0,149$ & 0,412 & $-0,512$ & $-0,383$ & 0,771 \\
\hline WAB / WT + Mais & 0,376 & $-0,045$ & 0,391 & $-0,162$ & 1,315 & $-0,526$ & 0,368 & 0,013 \\
\hline$S A B$ & $-0,496$ & $-0,288$ & $-0,546$ & $-0,207$ & 0,589 & 0,612 & 1,289 & $-0,834$ \\
\hline Einj. Blühm. & $-0,495$ & $-0,181$ & $-0,603$ & 0,275 & $-0,828$ & $-0,002$ & $-0,407$ & $-0,690$ \\
\hline Amarant & $-0,293$ & 0,014 & $-0,367$ & 0,317 & $-0,820$ & 0,358 & $-0,008$ & $-0,111$ \\
\hline Mais & 0,268 & 0,424 & 0,424 & $-0,069$ & $-0,203$ & 0,222 & $-0,565$ & 0,774 \\
\hline Amarant / Mais & 0,187 & 0,361 & 0,312 & 0,127 & $-0,428$ & $-0,019$ & $-0,499$ & 0,438 \\
\hline Deut. Weidelgras & n.v. & n.v. & n.v. & n.v. & n.v. & n.v. & n.v. & n.v. \\
\hline Mehrj. Blühm. & $-0,514$ & $-0,048$ & $-0,583$ & 0,187 & $-0,846$ & 0,300 & $-0,205$ & $-0,484$ \\
\hline Durchw. Silphie & n.v. & n.v. & n.v. & n.v. & n.v. & n.v. & n.v. & n.v. \\
\hline \multicolumn{9}{|c|}{2016} \\
\hline WIR + Mais & 0,220 & $-0,043$ & 0,283 & $-0,255$ & $-0,347$ & 0,178 & $-0,371$ & $-0,288$ \\
\hline WAB + Mais & 0,226 & $-0,270$ & 0,208 & $-0,786$ & 0,132 & 0,074 & 0,563 & $-0,108$ \\
\hline WT + Mais & 0,384 & $-0,126$ & 0,454 & $-0,363$ & $-0,132$ & $-0,146$ & $-0,634$ & 0,176 \\
\hline WAB / WT + Mais & 0,291 & $-0,130$ & 0,312 & $-0,247$ & 1,455 & 0,241 & 0,181 & $-0,252$ \\
\hline$S A B$ & $-0,289$ & $-0,369$ & $-0,349$ & 0,059 & 1,596 & 0,138 & 1,142 & $-0,157$ \\
\hline Einj. Blühm. & $-0,726$ & $-0,135$ & $-0,781$ & 0,168 & $-0,772$ & $-0,072$ & $-0,130$ & $-1,348$ \\
\hline Amarant & $-0,389$ & $-0,163$ & $-0,444$ & 0,136 & $-0,863$ & 0,042 & 0,020 & $-0,376$ \\
\hline Mais & 0,228 & 0,355 & 0,402 & $-0,091$ & $-0,423$ & $-0,354$ & $-0,716$ & 0,933 \\
\hline Amarant / Mais & 0,195 & 0,390 & 0,355 & $-0,067$ & $-0,635$ & $-0,303$ & $-0,666$ & 0,596 \\
\hline Deut. Weidelgras & 0,321 & $-0,117$ & 0,316 & 0,579 & 0,641 & 0,432 & 0,620 & 1,332 \\
\hline Mehrj. Blühm. & $-0,455$ & 0,421 & $-0,541$ & 0,500 & $-0,742$ & $-0,126$ & 0,021 & $-0,700$ \\
\hline Durchw. Silphie & $-0,006$ & 0,187 & $-0,216$ & 0,367 & 0,089 & $-0,105$ & $-0,030$ & 0,191 \\
\hline
\end{tabular}

$\mathrm{WIR}=$ Wickroggen, $\mathrm{WAB}=$ Winterackerbohnen, $\mathrm{WT}=$ Wintertriticale, $\mathrm{SAB}=$ Sommerackerbohnen, n.v. = Werte nicht verfügbar.

lagen die Winterackerbohnen sowie das Gemenge aus Winterackerbohnen und Wintertriticale jeweils gefolgt von Mais höher als Mais in Hauptfruchtstellung mit 0,268. Die geringsten Werte für den Teilindex Trockenmasseertrag zeigten im ersten Versuchsjahr die Blühmischungen und die Sommerackerbohnen mit Werten zwischen -0,495 und -0,514.

Im zweiten Versuchsjahr waren die Indexwerte beim Teilindex Trockenmasseertrag bei den Varianten des Zweikulturnutzungssystems bis auf die Wintertriticale gefolgt von Mais etwa vergleichbar mit Mais als Sommerhauptfrucht mit 0,220 bis 0,291. Das Deutsche Weidelgras war mit 0,321 höher als diese Varianten. Den mit Abstand geringsten Indexwert hatte im zweiten Versuchsjahr die einjährige Blühmischung mit -0,726. Aber auch die mehrjährige Blühmischung, der Amarant und die Sommerackerbohnen hatten Indexwerte im negativen Wertebereich. 


\section{Teilindex TS-Gehalt}

Ähnlich dem Standort Reinshof hatten auch am Standort Schoningen der Mais und das Gemenge aus Amarant und Mais in beiden Versuchsjahren mit 0,424 und 0,361 bzw. 0,355 und 0,390 hohe positive Werte beim Teilindex TS-Gehalt (Tabelle 20). Im zweiten Versuchsjahr reihte sich hier auch die mehrjährige Blühmischung mit 0,421 ein. Die geringsten Indexwerte zeigten in beiden Versuchsjahren mit - 0,288 bzw. - 0,369 die Sommerackerbohnen. Im zweiten Versuchsjahr fiel auf, dass der überwiegende Teil der Varianten negative Indexwerte hatte, darunter auch Varianten, die im ersten Versuchsjahr im positiven Wertebereich waren (Zweikulturnutzungssystem Wintertriticale + Mais, Amarant in Reinsaat).

\section{Teilindex Methan}

Die Variante mit den höchsten Werten beim Teilindex Trockenmasseertrag, Wintertriticale gefolgt von Mais, zeigte am Standort Schoningen auch die höchsten Werte beim Teilindex Methan mit 0,682 im ersten und 0,454 im zweiten Versuchsjahr und war damit vor allem im ersten Versuchsjahr deutlich besser als Mais mit 0,424 bzw. 0,402 (Tabelle 20). Positive Indexwerte zeigten in beiden Versuchsjahren auch die übrigen Varianten des Zweikulturnutzungssystems, der Mais, das Gemenge aus Amarant und Mais sowie das im zweiten Versuchsjahr in die Bewertung eigehende Deutsche Weidelgras. Analog zum Teilindex Trockenmasseertrag hatten die Sommerackerbohnen, die Blühmischungen, der Amarant und die Durchwachsene Silphie negative Werte beim Teilindex Methan.

\section{Teilindex Nmin}

Den höchsten Wert für den Teilindex Nmin hatte im zweiten Versuchsjahr das Deutsche Weidelgras mit 0,579 (Tabelle 20). Ebenfalls positive Indexwerte zeigten in beiden Versuchsjahren die Blühmischungen und der Amarant sowie im Jahr der Untersuchung (2016) auch die Durchwachsene Silphie. Wie am Standort Reinshof hatte am Standort Schoningen die Variante Winterackerbohnen gefolgt von Mais des Zweikulturnutzungssystems in beiden Versuchsjahren mit -0,670 bzw. -0,786 den größten negativen Wert für den Teilindex Nmin. Daneben waren aber, anders als am Standort Reinshof, auch die übrigen Varianten des Zweikulturnutzungssystems hier in beiden Versuchsjahren im negativen Wertebereich. Bei den Sommerackerbohnen änderte sich der Indexwert vom ersten zum zweiten Versuchsjahr von einem negativen Wert in einen positiven, beim Gemenge aus Amarant und Mais war es umgekehrt.

\section{Teilindex Wurzel-Trockenmasse}

Die Indexwerte des Teilindex Wurzeltrockenmasse unterschieden sich auch am Standort Schoningen deutlich zwischen den Varianten. Den höchsten Wert erzielte im ersten Versuchsjahr das Gemenge aus Winterackerbohnen und Wintertriticale mit nachfolgendem Mais mit 1,315 (Tabelle 20). Auch im zweiten Versuchsjahr war diese Variante mit einem Indexwert von 1,455 sehr stark, wurde aber von den Sommerackerbohnen mit 1,596 überboten. Positive Indexwerte hatten in beiden Versuchsjahren auch die Winterackerbohnen mit nachfolgendem Mais sowie im zweiten Versuchsjahr das Deutsche Weidelgras und die Durchwachsene Silphie. Indexwerte im deutlich negativen Wertebereich zeigten in beiden Versuchsjahren die ein- und mehrjährige Blühmischung sowie der Amarant mit -0,742 bis $-0,863$. Wenn auch etwas besser als diese Varianten aber dennoch mit negativen Indexwerten schnitten der Mais und das Gemenge aus Amarant und Mais ab. 


\section{Teilindex Bodenwasser}

Wie auch am Standort Reinshof, gab es am Standort Schoningen beim Teilindex Bodenwasser Unterschiede zwischen den Versuchsjahren (Tabelle 20). So hatten einige Varianten im ersten Versuchsjahr negative Indexwerte und im zweiten Versuchsjahr positive, andere Varianten zuerst positive und im Jahr darauf negative. Auch bei der Rangfolge der Indexwerte zwischen den Varianten in den beiden Versuchsjahren gab es nur wenig Übereinstimmung. Im ersten Versuchsjahr hatten die Sommerackerbohnen mit 0,612 den höchsten und mit $-0,526$ das Gemenge aus Winterackerbohnen und Wintertriticale gefolgt von Mais den geringsten Indexwert. Im zweiten Versuchsjahr hingegen hatte das Deutsche Weidelgras mit 0,432 den höchsten Indexwert und der Mais, der im ersten Versuchsjahr im positiven Wertebereich angesiedelt war, mit -0,354 den geringsten Indexwert.

\section{Teilindex Spurenelemente}

Deutliche Unterschiede zwischen den Varianten gab es auch beim Teilindex Spurenelemente am Standort Schoningen (Tabelle 20). Mit weitem Abstand waren die Sommerackerbohnen mit 1,289 und 1,142 im ersten wie auch im zweiten Versuchsjahr die Variante mit den höchsten Indexwerten. Darauf folgten im ersten Versuchsjahr die Varianten des Zweikulturnutzungssystems, bei denen Winterackerbohnen Teil der Biomasse waren, die Winterackerbohnen-Reinsaat und das Gemenge aus Winterackerbohnen und Wintertriticale jeweils gefolgt von Mais mit 0,409 bzw. 0,368. Im zweiten Versuchsjahr war das Zweikulturnutzungssystem aus Winterackerbohnen und Mais ebenfalls recht stark mit 0,563, wurde aber vom Deutschen Weidelgras mit 0,620 übertroffen. Die geringsten Werte für diesen Teilindex hatte in beiden Versuchsjahren der Mais mit -0,565 bzw. -0,716. Auch das Gemenge aus Amarant und Mais sowie das Zweikulturnutzungssystem aus Wintertriticale und Mais waren nur wenig höher als der Mais in Reinsaat.

\section{Teilindex Ökonomische Aspekte}

Deutlich größer als am Standort Reinshof waren die Unterschiede zwischen den Varianten beim Teilindex Ökonomische Aspekte am Standort Schoningen. Im ersten Versuchsjahr hatten der Mais mit 0,774 und das Zweikulturnutzungssystem aus Wintertriticale und Mais mit 0,771 die höchsten Werte für diesen Teilindex (Tabelle 20). Auch das Gemenge aus Amarant und Mais war in diesem Versuchsjahr recht stark mit 0,438. Im zweiten Versuchsjahr, in dem auch das Deutsche Weidelgras an diesem Standort in die ökonomische Bewertung einging, überstieg das Deutsche Weidelgras alle anderen Varianten mit einem Indexwert von 1,332. Es folgte die Mais-Reinsaat mit 0,933. Das Gemenge aus Amarant und Mais $(0,596)$, ganz besonders aber das Zweikulturnutzungssystem aus Wintertriticale und Mais blieb in diesem Versuchsjahr mit 0,176 weit dahinter zurück.

Geringe Werte beim Teilindex ökonomische Aspekte zeigten im ersten Versuchsjahr die Sommerackerbohnen mit -0,834 und die einjährige Blühmischung mit -0,690. Im zweiten Versuchsjahr wurde bei der einjährigen Blühmischung, bedingt durch den hier erzielten negativen Deckungsbeitrag, mit $-1,348$ einen Indexwert kleiner als -1 berechnet. Ebenfalls recht geringe Indexwerte wurden bei der mehrjährigen Blühmischung ermittelt. 


\subsubsection{Teilindex Soziale Aspekte (Akzeptanz)}

Die Indexwerte für den Teilindex Soziale Aspekte, berechnet aus den Umfrageergebnissen zur Akzeptanz des Energiepflanzenanbaus in der Bevölkerung, waren mit 0,452 bzw. 0,353 am höchsten bei der mehrjährigen und einjährigen Blühmischung (Tabelle 21). Positive Indexwerte zeigten bei diesem Teilindex auch die Zweikulturnutzungssysteme aus Wickroggen und dem Gemenge aus Winterackerbohnen und Wintertriticale jeweils gefolgt von Mais sowie das Deutsche Weidelgras und die Durchwachsene Silphie. Mit -0,336 war der Mais die Variante mit dem geringsten Wert für den Teilindex soziale Aspekte. Aber auch das Gemenge aus Amarant und Mais $(-0,262)$, die Amarant-Reinsaat $(-0,254)$ und das Zweikulturnutzungssystem aus Wintertriticale und Mais $(-0,196)$ hatten Indexwerte im deutlich negativen Wertebereich.

Tabelle 21: Teilindex Soziale Aspekte (Akzeptanz) der untersuchten Feldfrüchte.

\begin{tabular}{lc}
\hline Variante & Teilindex soz. Aspekte \\
\hline Wickroggen + Mais & 0,177 \\
Winterackerbohnen (WAB) + Mais & $-0,012$ \\
Wintertriticale (WT) + Mais & $-0,196$ \\
Gemenge WAB / WT + Mais & 0,095 \\
Sommerackerbohnen & $-0,090$ \\
Einjährige Blühmischung & 0,353 \\
Amarant & $-0,254$ \\
Mais & $-0,336$ \\
Gemenge Amarant / Mais & $-0,262$ \\
Deutsches Weidelgras & 0,025 \\
Mehrjährige Blühmischung & 0,452 \\
Durchwachsene Silphie & 0,046 \\
\hline
\end{tabular}

\subsubsection{Gesamtindex IrA an den Standorten Reinshof und Schoningen}

In Abbildung 23 und Abbildung 24 sind die Teilindizes und der Index der relativen Anbauwürdigkeit, IrA, für die beiden Standorte, Reinshof und Schoningen als Mittel über die Versuchsjahre 2015 und 2016 aggregiert dargestellt.

An beiden Standorten zeigte das Deutsche Weidelgras den höchsten Wert für den Index der relativen Anbauwürdigkeit. Am Standort Reinshof ergab sich ein IrA-Wert von etwa 6,5, am Standort Schoningen von etwa 8,9. An beiden Standorten war bei dieser Variante die überwiegende Anzahl der Teilindizes im positiven Wertebereich. Am Standort Reinshof waren zwar die Teilindizes TM-Ertrag, TS-Gehalt und Methan im negativen Wertebereich, hatten aber im Vergleich mit den anderen Teilindizes nur wenig Gewicht (Abbildung 23). Seine Stärken zeigte das Deutsche Weidelgras hier vor allem bei Nmin, der Wurzeltrockenmasse und den Spurenelementen, sichtbar geworden durch die hohen Teilindexwerte, die diese Parameter repräsentieren.

Am Standort Schoningen war, wie am Standort Reinshof auch, der Teilindex TS-Gehalt beim Deutschen Weidelgras negativ, außer diesem hier jedoch kein weiterer Teilindex (Abbildung 24). Die größte Stärke des Deutschen Weidelgrases am Standort Schoningen war die ökonomische Bewertung. Der Teilindex ökonomische Aspekte hatte hier am Gesamtindex IrA das 
größte Gewicht aller Teilindizes. Aber auch beim Nmin, der Wurzeltrockenmasse, dem Bodenwassergehalt und den Spurenelementen zeigte sich das Deutsche Weidelgras im Vergleich mit den anderen Varianten als günstige Feldfrucht. Allerdings gingen beim Deutschen Weidelgras an diesem Standort nur die Daten des zweiten Versuchsjahres ein.

\section{Index}

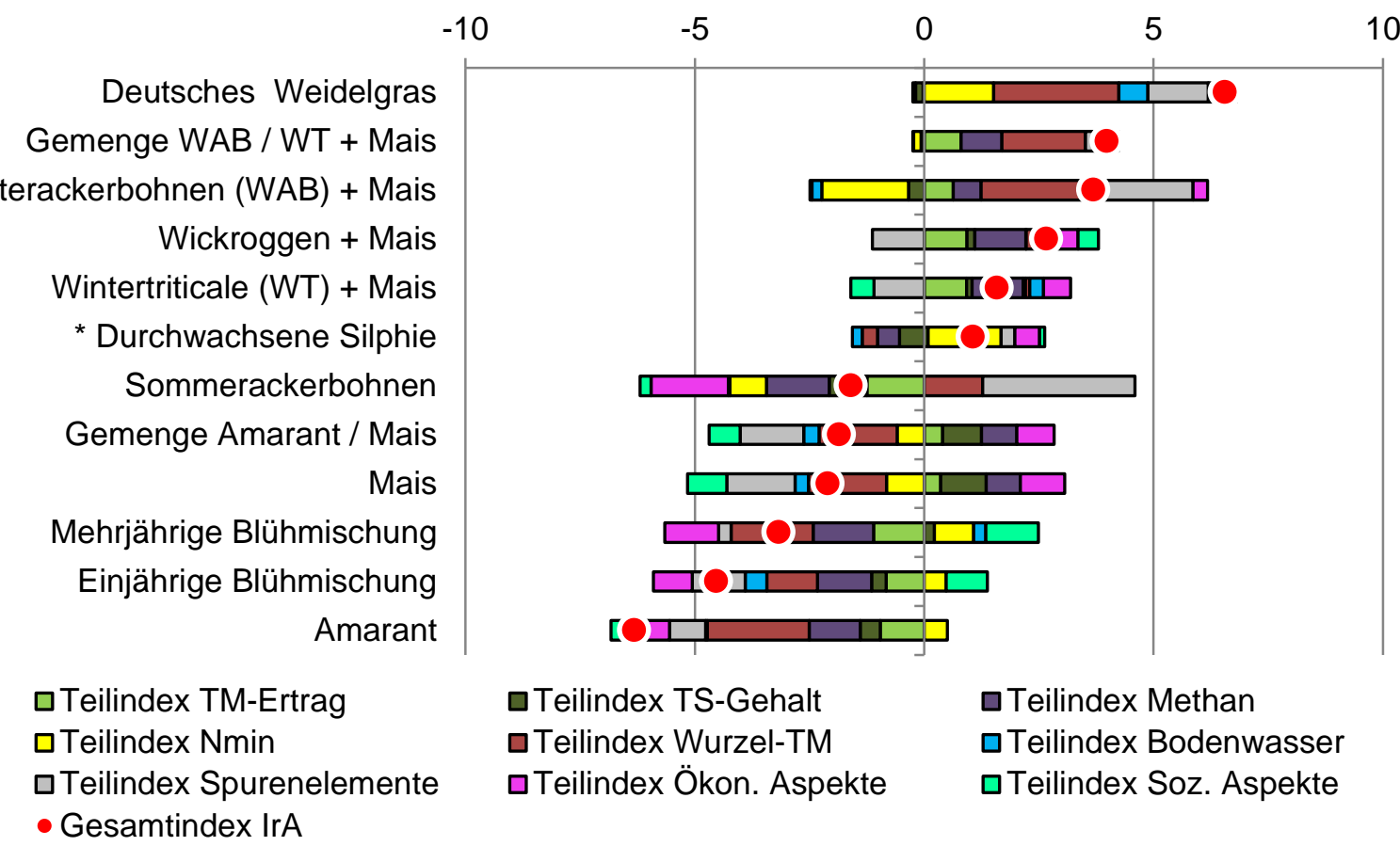

Abbildung 23: Teilindizes und Gesamtindex der relativen Anbauwürdigkeit (IrA). Mittelwerte aus den Versuchsjahren 2015 und 2016 am Standort Reinshof. Bei der mit * gekennzeichneten Variante geht nur das Versuchsjahr 2016 ein. Dargestellt sind die skalierten Werte für die Teilindizes sowie die daraus gebildete Summe (Gesamtindex IrA).

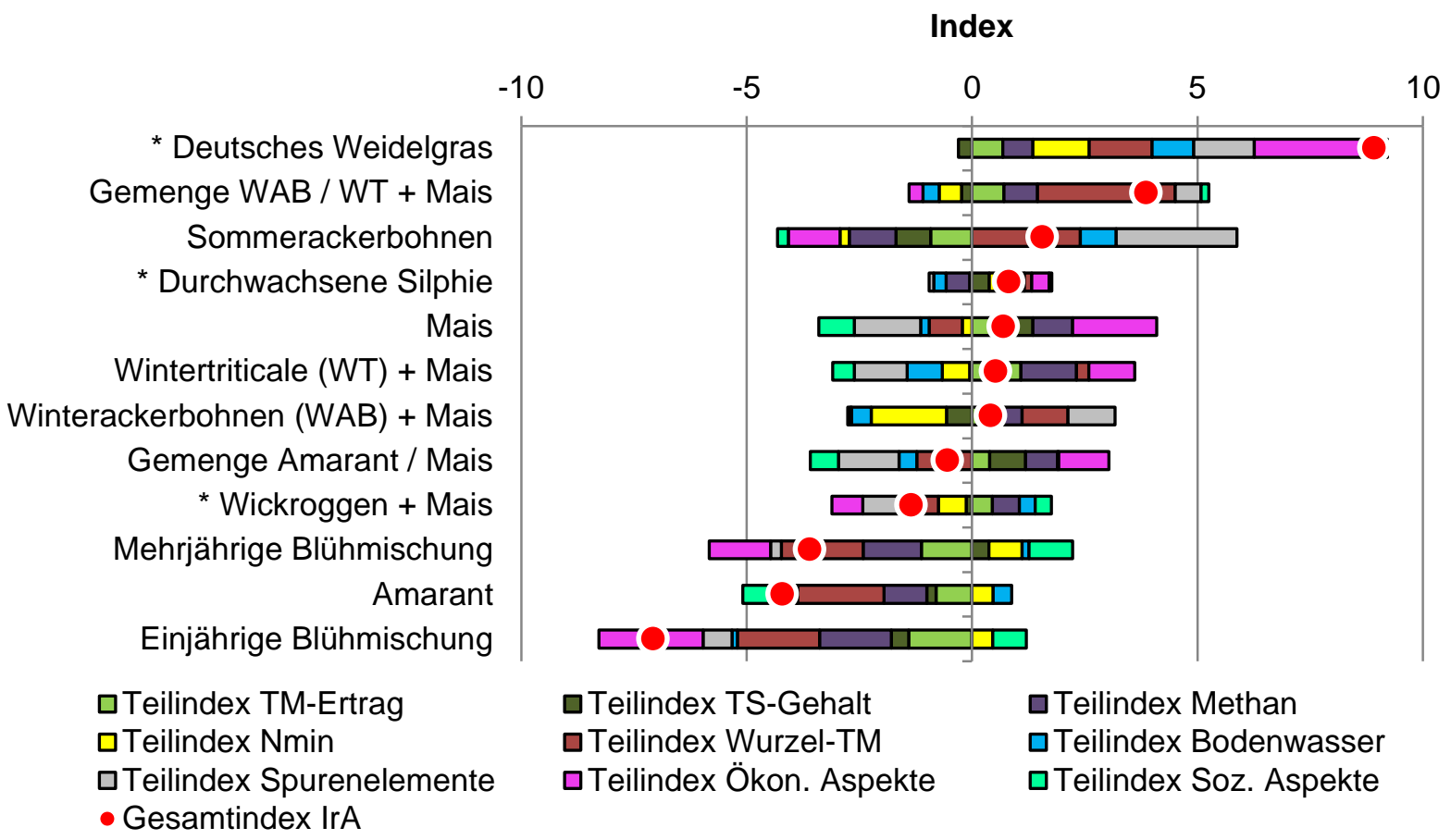

Abbildung 24: Teilindizes und Gesamtindex der relativen Anbauwürdigkeit (IrA). Mittelwerte aus den Versuchsjahren 2015 und 2016 am Standort Schoningen. Bei den mit * gekennzeichneten Varianten geht nur das Versuchsjahr 2016 ein. Dargestellt sind die skalierten Werte für die Teilindizes sowie die daraus gebildete Summe (Gesamtindex IrA). 
Bei der Rangfolge der Varianten, absteigend sortiert nach den Werten für den Gesamtindex IrA, folgten am Standort Reinshof die Zweikulturnutzungssysteme beginnend mit dem Gemenge aus Winterackerbohnen und Wintertriticale über die Winterackerbohnen in Reinsaat, den Wickroggen bis hin zur Wintertriticale jeweils gefolgt von Mais mit IrA-Werten von 4,0 bis 1,6 (Abbildung 23). Bei den Winterackerbohnen waren die Teilindizes Wurzel-Trockenmasse und Spurenelemente sehr stark. Die Schwäche beim Nmin und dem entsprechend negativen Teilindex Nmin stand diesen jedoch entgegen. Einen ebenfalls positiven Wert für den Gesamtindex IrA hatte am Standort Reinshof mit etwa 1,1 die Durchwachsene Silphie, die sich durch einen hohen Wert beim Teilindex Nmin auszeichnete.

Am Standort Schoningen folgte auf das Deutsche Weidelgras ebenso wie am Standort Reinshof das Zweikulturnutzungssystem mit dem Gemenge aus Winterackerbohnen und Wintertriticale und Mais mit einem IrA-Wert von etwa 3,9 (Abbildung 24). Besonders hervor hob sich hier der Teilindex Wurzel-TM. Wenn auch weniger gewichtig als dieser Teilindex aber ebenfalls positiv waren die Teilindizes TM-Ertrag, Methan, Spurenelemente und soziale Aspekte.

An dritter Stelle in der Rangfolge der Varianten ordneten sich am Standort Schoningen die Sommerackerbohnen ein. Obwohl hier die Mehrzahl der Teilindizes (TM-Ertrag, TS-Gehalt, Methan, Nmin, ökonomische Aspekte, soziale Aspekte) im negativen Wertebereich lagen, war der Gesamtindex IrA mit etwa 1,6 positiv (Abbildung 24). Die hohen Werte bei den Teilindizes Wurzel-TM und Spurenelemente hoben die negativen Teilindizes auf. Nur knapp positiv waren mit etwa 0,8 bis 0,4 am Standort Schoningen die IrA-Werte bei der Durchwachsenen Silphie, dem Mais sowie den Zweikulturnutzungssystemen aus Wintertriticale und Winterackerbohnen jeweils gefolgt von Mais. Bei diesen Varianten sowie dem Gemenge aus Amarant und Mais mit einem Wert für den Gesamtindex IrA von etwa $-0,5$ hatten die Teilindizes im positiven wie auch im negativen Wertebereich etwa das gleiche Gewicht. Bei Mais, dem Zweikulturnutzungssystem aus Wintertriticale und Mais sowie dem Gemenge aus Amarant und Mais fiel im negativen Wertebereich besonders der Teilindex Spurenelemente auf. Bei diesen Varianten war im positiven Wertebereich der Teilindex ökonomische Aspekte vergleichsweise groß.

Am Standort Reinshof lag, anders als am Standort Schoningen, der Wert für den Gesamtindex IrA der Sommerackerbohnen mit etwa -1,6 im deutlich negativen Bereich (Abbildung 23). Sehr hohe positive Werte hatte hier zwar der Teilindex Spurenelemente und auch der Teilindex Wurzel-TM, aber auch die Teilindizes im negativen Bereich und dabei vor allem die Teilindizes TM-Ertrag, Methan und ökonomische Aspekte hatten vergleichsweise hohe Werte. Negative Werte für den Gesamtindex IrA zeigten auch das Gemenge aus Amarant und Mais, der Mais sowie die ein- und mehrjährige Blühmischung. Der Mais und das Gemenge aus Amarant und Mais ähnelten sich bei der Verteilung der Teilindizes im positiven und negativen Wertebereich stark. So zeigten diese Varianten positive Werte bei den Teilindizes TM-Ertrag, TS-Gehalt, Methan und ökonomische Aspekte. Alle übrigen waren negativ, wobei besonders die Teilindizes Wurzel-TM und Spurenelemente großes Gewicht am Gesamtindex IrA hatten. Bei der einund mehrjährigen Blühmischung war die überwiegende Anzahl der Teilindizes im negativen Wertebereich. Positiv zu Buche schlugen mit relativ hohem Gewicht nur die Teilindizes Nmin und soziale Aspekte. Den geringsten Wert für den Index der relativen Anbauwürdigkeit hatte am Standort Reinshof mit etwa -6,3 der Amarant. Hier waren bis auf den Teilindex Nmin alle Teilindizes im negativen Wertebereich. 
Im Gegensatz zum Standort Reinshof, an dem das Zweikulturnutzungssystem aus Wickroggen und Mais mit einem positiven IrA-Wert einen der oberen Ränge besetzte, hatte diese Variante am Standort Schoningen mit etwa -1,3 einen negativen IrA-Wert (Abbildung 24). Deutlich dahinter und ähnlich dem Standort Reinshof eingeordnet, lagen die mehrjährige Blühmischung und der Amarant mit IrA-Werten von -3,6 und -4,2. Besonders hohe negative Werte hatten diese Varianten beim Teilindex Wurzel-TM, aber auch bei den Teilindizes TM-Ertrag und Methan und bei der mehrjährigen Blühmischung zusätzlich beim Teilindex ökonomische Aspekte. Mit etwa -7,1 war am Standort Schoningen die einjährige Blühmischung mit deutlichem Abstand die Variante mit dem geringsten Wert für den Index der relativen Anbauwürdigkeit. Hier zeigten die Teilindizes TM-Ertrag, Methanausbeute, Wurzel-TM und besonders der Teilindex ökonomische Aspekte deutlich negative Werte. 


\subsection{Teilindizes des Index der relativen Anbauwürdigkeit (IrA)}

\subsubsection{Teilindex Trockenmasseertrag}

Der Teilindex Trockenmasseertrag der oberirdischen Biomasse dient der Bewertung der Leistungsfähigkeit von Energiepflanzen für die Biogaserzeugung. Der Trockenmasseertrag ist hier eine wichtige Kenngröße, da er zusammen mit dem spezifischen Methanertrag die Menge an produzierten Biogas je Flächeneinheit bestimmt. Der Trockenmasseertrag ist dabei die entscheidende Größe (Herrmann \& Rath 2012). Für eine effiziente Flächennutzung zur Biogaserzeugung sind dementsprechend Varianten mit besonders hohen Erträgen positiv zu bewerten.

Hohe Werte für den aus der Summe der Erst- und Zweitfruchterträge konstruierten Teilindex Trockenmasseertrag hatten die Varianten des Zweikulturnutzungssystems (Tabelle 19, Tabelle 20). Insbesondere im zweiten Versuchsjahr am Standort Reinshof hoben sich diese Varianten deutlich von den anderen ab und hatten - zumindest allein mit Blick auf den Teilindex Trockenmasseertrag - eine bis zu vierfach höhere Anbauwürdigkeit als der alleinige Anbau von Mais oder dem Gemenge aus Amarant und Mais mit ebenfalls vergleichsweise hohen positiven Indexwerten. Mit mehr als $300 \mathrm{dt} \mathrm{TM} \mathrm{ha}{ }^{-1}$ zeigten die Varianten des Zweikulturnutzungssystems am Standort Reinshof ein sehr hohes Ertragspotenzial, das auch die Ergebnisse anderer Untersuchungen zu Zweikulturnutzungssystemen z.T. weit übersteigt (Schittenhelm et al. 2011, Graß et al. 2013). Mit Höchsterträgen dieser Varianten von $230 \mathrm{dt} \mathrm{TM}$ ha $^{-1}$ am Standort Schoningen zeigt sich aber auch die Standortabhängigkeit des Systems.

Im zweiten Versuchsjahr brachen die Erträge der Erstfrüchte des Zweikulturnutzungssystems im Vergleich zum ersten Versuchsjahr etwas ein (Abbildung 4, Abbildung 7). Bei Wiederholbarkeit der Erstfrucht-Erträge des ersten Versuchsjahres im zweiten Versuchsjahr, wäre zumindest am Standort Reinshof ein noch höherer Gesamttrockenmasseertrag des Zweikulturnutzungssystems möglich gewesen. Die Unterschiede bei den Trockenmasseerträgen der Erstfrüchte der beiden Versuchsjahre hingen möglicherweise auch mit der unterschiedlichen Vorgehensweise bei der Anlage der Feldversuche in den beiden Versuchsjahren zusammen. So wurde zur Aussaat der Erstfüchte des ersten Versuchsjahres der Boden gepflügt, zur Aussaat der Erstfrüchte des zweiten Versuchsjahres jedoch nicht. Es erfolgte eine reduzierte Bodenbearbeitung, bei der viele Ernterückstände der Vorfrucht an der Bodenoberfläche verblieben. Am Standort Reinshof wurde vor den Erstfrüchten des zweiten Versuchsjahres zudem bereits in zwei aufeinanderfolgenden Jahren Winterweizen angebaut, vor der Aussaat der Erstfrüchte des ersten Versuchsjahres dagegen nur ein Mal. Als Vor-Vorfrucht waren hier Zuckerrüben angebaut. Am Standort Schoningen war die Vorfrucht im ersten Versuchsjahr Winterraps und im zweiten Versuchsjahr Winterweizen. Ein möglicherweise ertragswirksamer Unterschied zwischen den Erstfruchtbeständen des ersten und zweiten Versuchsjahres bestand aber in der Verunkrautung im Spätherbst bzw. zu Vegetationsbeginn im Frühjahr. Während im ersten Versuchsjahr nahezu kein Unkraut und Ungras zu finden war, war der Deckungsgrad insbesondere mit der Geruchlosen Kamille, dem Einjährigem Rispengras und Ausfallgetreide 
sowie zusätzlich am Standort Schoningen auch Ackerfuchsschwanz (Alopecurus myosuroides) im zweiten Versuchsjahr sehr groß (Abbildung 6). Chemische oder mechanische Maßnahmen zur Unkrautregulierung wurden erst nach Vegetationsbeginn im Frühjahr durchgeführt.

Die Trockenmasseerträge der Erstfrüchte lagen am Standort Reinshof, bis auf die Winterackerbohnen, die etwas schwächer waren, innerhalb der beiden Versuchsjahre auf einem vergleichbaren Niveau (Abbildung 4). Zur Bildung der Biomasse kamen jedoch ungleich hohe NDüngermengen zum Einsatz. So stand nach Berücksichtigung der Nmin-Mengen zu Vegetationsbeginn, ganzflächiger Gärrestdüngung und mineralischer N-Düngung zur Erreichung des angestrebten Düngeniveaus, dem Wickroggen etwa $130 \mathrm{~kg} \mathrm{~N} \mathrm{ha}^{-1}$ und der Wintertriticale etwa $180 \mathrm{~kg} \mathrm{~N} \mathrm{ha}^{-1}$ zur Verfügung. Bei den Winterackerbohnen und dem Gemenge aus Winterackerbohnen und Wintertriticale erfolgte keine zusätzliche Mineraldüngergabe. Die Winterackerbohnen fanden am Standort Reinshof aus Nmin zu Vegetationsbeginn und Gärrestdüngung 94 (erstes Versuchsjahr) bzw. $116 \mathrm{~kg} \mathrm{~N}^{-1}$ (zweites Versuchsjahr) vor; beim Gemenge aus Winterackerbohnen und Wintertriticale waren es 77 bzw. $103 \mathrm{~kg} \mathrm{~N} \mathrm{ha}^{-1}$. Dass der N-Düngebedarf der Wintertriticale auch der N-Aufnahme durch die Pflanzen entsprach, zeigt Abbildung 10. Die vorgesehene N-Menge von $180 \mathrm{~kg} \mathrm{~N} \mathrm{ha}^{-1}$ wurde in etwa auch in der oberirdischen Biomasse der Wintertriticale akkumuliert. Wie die Daten zeigen, war zur Bildung von Trockenmasseerträgen, die mit denen der optimal mit Stickstoff versorgten Wintertriticale-Reinsaat vergleichbar waren, beim Gemenge aus Winterackerbohnen und Wintertriticale keine zusätzliche mineralische Düngung nötig. Auch der Wickroggen benötigte deutlich weniger N-Dünger als die Wintertriticale-Reinsaat. So wurde der hohe Wert für den Teilindex Trockenmasseertrag bei der Variante Wintertriticale gefolgt von Mais mit einem sehr hohen N-Düngemitteleinsatz erkauft. Die N-Düngung des Maises als Zweitfrucht war nach Berücksichtigung der NminMengen nach der Ernte der Erstfrüchte (Daten nicht dargestellt) bei diesen drei Varianten etwa gleich, weshalb hier die Erstfrüchte den Unterschied in der Düngemenge bewirken.

Es wird deutlich, dass mit dem Anbau von Gemengen aus Leguminosen und Nicht-Leguminosen als Energiepflanzen ein enormes N-Düngemittel-Einsparpotenzial bei der Erzeugung von Biomasse zur Biogaserzeugung besteht. Zu dem Ergebnis kommen auch Graß et al. (2013) bei einer Untersuchung von Winterroggen in Reinsaat sowie im Gemenge mit Wintererbse als Erstfrüchte im Zweikulturnutzungssystem zur Biomasseerzeugung. Weniger eindeutig waren dagegen die Ergebnisse von Karpenstein-Machan \& Stülpnagel (2000). Wenn auch die ungedüngten Gemenge aus Winterroggen und Wintererbsen bzw. Winterroggen und Inkarnatklee als Erstfrüchte im Zweikulturnutzungssystem bis zur Ernte im Juni in einem Versuchsjahr mit dem Trockenmasseertrag der mit Stickstoff gedüngten Winterroggen-Reinsaat gleichzogen bzw. diesen deutlich überstiegen, war das im Mittel über die Versuchsjahre nicht der Fall. So zeigte es sich auch am Standort Schoningen im hier vorliegenden Versuch, an dem in beiden Versuchsjahren die mit Stickstoff gedüngte Wintertriticale-Reinsaat höhere Trockenmasseerträge erzielte als die geringer mit Stickstoff gedüngten Erstfüchte, bei denen aber Leguminosen in Reinsaat oder als Gemengepartner angebaut wurden. Um das N-Düngemittel-Einsparpotenzial zu bewerten, wäre ein weiterer Teilindex denkbar, der die Ressourcennutzung bzw. den Ressourcenschutz aufgreift. Positiv zu Buche schlagen würde dabei auch die in dieser Untersuchung durchgeführte reduzierte N-Düngung der Zweitfrucht Mais nach Winterackerbohnen bei Berücksichtigung der Nmin-Mengen nach der Ernte, die bei Winterackerbohnen 
höher waren im Vergleich mit den übrigen Erstfrüchten (Daten nicht dargestellt). In einem solchen Teilindex könnten z.B. N-Transferraten im Gemenge aus Leguminosen und nicht NichtLeguminosen sowie die Vorfruchtwirkung von Leguminosen in Form von N-Düngemittel-Äquivalenten Eingang finden, wie Köpke \& Nemecek (2010) vorgeschlagen haben.

Neugschwandtner et al. (2015) konnten bei der Winterackerbohnen-Reinsaat N-Mengen aus der $\mathrm{N}_{2}$-Fixierung bis zur Kornreife von bis zu $219 \mathrm{~kg} \mathrm{~N} \mathrm{ha}^{-1}$ finden. Bis zur Vollblüte, kurz nach der die Winterackerbohnen in der vorliegenden Untersuchung geerntet wurden, ist dies mit 90 bis $100 \mathrm{~kg} \mathrm{~N}^{-1}$ bei unterschiedlichen Winterackerbohnen-Genotypen deutlich weniger, wie Siebrecht-Schöll (2019) herausfand. Menke (2011) stellte bei der Ernte im Mai stark schwankende N-Mengen aus symbiotischer $\mathrm{N}_{2}$-Fixierung von 252 und $55 \mathrm{~kg} \mathrm{~N}^{-1}$ in unterschiedlichen Versuchsjahren bei Winterackerbohnen fest. Welche N-Mengen von den Ackerbohnen im vorliegenden Versuch aus der Luft fixiert wurden und welche aus dem Boden aufgenommen wurden, lässt sich nur schwer abschätzen, da es keine ungedüngte Variante gab. Die N-Mengen in der oberirdischen Biomasse bei der Winterackerbohnen-Reinsaat (200-250 kg N ha-1) sowie dem Gemenge aus Winterackerbohnen und Wintertriticale (180-220 kg N ha-1) waren zumindest weitaus höher als zu Vegetationsbeginn über Nmin und Gärrest zur Verfügung stand, sodass auch hier von einem Beitrag zur Stickstoffversorgung aus symbiotischer $\mathrm{N}_{2}-$ Fixierung ausgegangen werden kann. Der im Versuch angebaute Wickroggen hatte mit $10 \%$ an der Aussaatmenge nur einen geringen Anteil Zottelwicken. Zander et al. (2018) gehen bei ihrer Praxisempfehlung von einer $\mathrm{N}_{2}$-Fixierung je nach Wickenanteil im Bestand von 20 bis 60 $\mathrm{kg} \mathrm{N} \mathrm{ha}^{-1}$ aus.

Anders als bei den Erstfrüchten des Zweikulturnutzungssystems konnte zumindest am Standort Reinshof die Zweitfrucht Mais im zweiten Versuchsjahr deutlich höhere Trockenmasseerträge erzielen als im ersten Versuchsjahr (Abbildung 4) und war damit ausschlaggebend für die hohen positiven Werte beim Teilindex Trockenmasseertrag. Die verfügbare Wachstumszeit der Maispflanzen wird durch die standortspezifischen Temperaturen, erfasst als Wärmesummen, bestimmt. Diese sind entscheidend für den Grad der Abreife und die Ertragsbildung. Die Basistemperatur, ab der Mais wächst und sich entwickelt, beträgt dabei $8^{\circ} \mathrm{C}$ (Herrmann et al. 2011). Laurenz (2000) geht von einer Basistemperatur von $6^{\circ} \mathrm{C}$ aus und gibt, ausgehend davon und unter Annahme von sonst günstigen Bedingungen hinsichtlich der Wasserversorgung, nötige Wärmesummen zur Abschätzung von Ernteterminen verschiedener SilomaisReifegruppen an. Im ersten Versuchsjahr wurde im vorliegenden Versuch als Zweitfrucht die Maissorte Simpatico mit einer Siloreifezahl von S 250 angebaut. Für diese, der mittelfrühen Reifegruppe zuzuordnenden Sorte, wäre laut Laurenz (2000) zur Erreichung eines TS-Gehalts von $32 \%$ eine Wärmesumme von $1490{ }^{\circ} \mathrm{Cd}$ nötig. Von der Aussaat bis zur Ernte, die am Standort Reinshof bestimmt war durch das erste Frostereignis, betrugen die Wärmesummen bei Annahme einer Basistemperatur von $6{ }^{\circ} \mathrm{C} 1288$ (Reinshof) bzw. $1214{ }^{\circ} \mathrm{Cd}$ (Schoningen). Dies ist deutlich weniger als laut Laurenz (2000) zur Erreichung der Siloreife notwendig wäre. Dass dies auch für den vorliegenden Versuch zutrifft, zeigen die geringen TS-Gehalte von nur $22 \%$ an beiden Standorten (Tabelle 7, Tabelle 8) sowie das bei der Ernte erreichte Entwicklungsstadium der Pflanzen, der Milchreife. Auch wenn den Pflanzen am Standort Schoningen eine um neun Tage längere Wachstumszeit zur Verfügung stand als am Standort Reinshof, war dies aufgrund des deutlich späteren Saattermins am Standort Schoningen nicht wirksam hinsichtlich der Wärmesummenakkumulation. 
Im zweiten Versuchsjahr wurden daher Maissorten mit deutlich geringerer Siloreifezahl und damit zusammenhängender kürzerer Entwicklungszeit und geringerem Wärmesummenbedarf gewählt. Am Standort Reinshof war es die Sorte Cathy mit einer Reifezahl von S 210 und am Standort Schoningen die Sorte P7326 mit einer Reifezahl von S 180. Für Sorten, die der frühen Reifegruppe angehören gibt Laurenz (2000) einen Wärmesummenbedarf von $1450{ }^{\circ} \mathrm{Cd}$ bei $6{ }^{\circ} \mathrm{C}$ Basistemperatur an. Im vorliegenden Versuch passte dies im zweiten Versuchsjahr für die Zeit von der Aussaat bis zur Ernte mit $1405^{\circ} \mathrm{Cd}$ am Standort Reinshof deutlich besser überein als im ersten Versuchsjahr. Zur Erreichung der Wärmesummen stand dem Mais im zweiten Versuchsjahr hier trotz einer etwas späteren Aussaat, eine um vier Tage längere Wachstumszeit, im Vergleich zum ersten Versuchsjahr, zur Verfügung. Durch die höhere Wärmesumme im zweiten Versuchsjahr und eine besser an die Vegetationszeit als Zweitfrucht angepassten Maissorte, konnte, wie an den hohen Trockenmasseerträgen ersichtlich, das Ertragspotenzial der Zweitfrucht am Standort Reinshof deutlich stärker ausgeschöpft werden als das im ersten Versuchsjahr der Fall war. Dies machte sich auch in der Bewertung der Varianten des Zweikulturnutzungssystems bemerkbar. Der Abstand der Werte des Teilindex Trockenmasseertrag zu Mais als Hauptfrucht konnte dadurch weiter ausgebaut werden.

Mit einem Aussaattermin am 21. Juni 2016 kam der Mais als Zweitfrucht am Standort Schoningen sehr spät in den Boden. Aufgrund der um vier Tage späteren Aussaat und um fünf Tage früheren Ernte im zweiten im Vergleich zum ersten Versuchsjahr stand dem Mais weniger Vegetationszeit als im ersten Versuchsjahr zur Verfügung. Die Vegetationszeit und die mit $1268{ }^{\circ} \mathrm{Cd}$ zustandegekommene Wärmesumme war wesentlich geringer als die bereits angesprochene, für eine gute Entwicklung und Abreife nötige Wärmesumme, was letztlich auch an den geringen Trockenmasseerträgen sichtbar wurde. Einschränkend sei aber auch auf die starke Trockenheit in den Sommermonaten des zweiten Versuchsjahres an diesem Standort hingewiesen. Trotz der geringen Wärmesumme wurde zumindest ein vergleichsweise hoher TS-Gehalt erreicht. Der Wert für den Teilindex Trockenmasseertrag konnte hier, anders als am Standort Reinshof, trotz einer angepassten Maissorte nicht verbessert werden, im Gegenteil. Die Trockenheit des zweiten Versuchsjahres außer Acht gelassen, legt dies den Schluss nahe, unter den Bedingungen dieses Standorts ein Anbausystem mit einer früher räumenden Winterzwischenfrucht, z.B. Grünschnittroggen und einer damit einhergehenden früheren Aussaat der Zweitfrucht, zu bevorzugen.

Auffällig waren auch die geringen Unterschiede bei den Trockenmasseerträgen der Zweitfrucht Mais nach den verschiedenen Erstfrüchten an den Standorten Reinshof und Schoningen im ersten Versuchsjahr. Vorfruchteffekte der verschiedenen Erstfrüchte, wie sie Menke (2011) fand mit einem deutlichen Ertragsvorteil des Maises nach Leguminosen im Vergleich zu NichtLeguminosen als Erstfrucht, gab es hier nicht. Allerdings wurde der Mais bei diesem Autor nicht gedüngt. In der hier vorliegenden Untersuchung wurde der Vorfruchteffekt offenbar durch die N-Düngung zu Mais auf ein einheitliches Niveau überdeckt. Das hat am Standort Reinshof auch den Anlass dazu gegeben, den im zweiten Versuchsjahr ermittelten Maisertrag nach Winterackerbohnen auf die fehlenden Parzellen der anderen Erstfrüchte zu übertragen.

Deutlich positive Werte beim Teilindex Trockenmasseertrag hatten in beiden Versuchsjahren und an beiden Standorten auch der Mais und das Gemenge aus Amarant und Mais (Tabelle 19, Tabelle 20). Voneinander ab hoben sich diese beiden Varianten aber nicht. Die Vorzüg- 
lichkeit von Gemengen aus Leguminosen und Nicht-Leguminosen hinsichtlich der Trockenmasseerträge im Vergleich der Reinsaaten ist hinreichend bekannt (Rauber et al. 2001, Yu 2016). Auch bei Gemengen aus Nicht-Leguminosen werden Vorteile gegenüber den Reinsaaten berichtet. Li et al. (2001) konnten z.B. einen Vorteil im Kornertrag beim Gemenge aus Weizen und Mais im Vergleich zu den Reinsaaten finden. Auch im Bereich der Biomasseproduktion zur energetischen Nutzung lassen sich Beispiele finden. So fanden Nassab et al. (2011) beim Anbau von Mais und Sonnenblumen als Energiepflanzen einen Wert für den Land Equivalent Ratio (LER) von größer eins. Ein erfolgreicher Gemengeanbau konnte aber auch für das Gemenge aus Amarant und Mais gezeigt werden: In einer Untersuchung fanden Ayodele \& Shittu (2013) an einem Gemenge aus Amarant zur Blattnutzung und Mais in Nigeria LER-Werte bei Variation verschiedener Faktoren (Düngung, Nutzungsverfahren des Amarants) von stets größer als eins. Das weist auf einen Vorteil des Gemenges gegenüber den Reinsaaten hin. Auch in Deutschland wurde bereits die Idee verfolgt, Amarant und Mais im Gemenge anzubauen. In diesem Fall ging es um die Qualitätsverbesserung der Maissilage zur Fütterung durch den im Amarant vorzufindenden hohen Gehalt essenzieller Aminosäuren (Sauerbeck 2004). Ein weiterer Aspekt des Gemengeanbaus von Amarant und Mais ist die Nutzung des Maises als Stützfrucht für den lageranfälligen Amarant (Förster et al. 2019). Ähnlich der Untersuchung von Sauerbeck (2004) zielt auch in der vorliegenden Untersuchung der Gemengeanbau von Amarant und Mais auf eine Verbesserung der Qualität im Vergleich zur reinen Maissilage ab - hier hinsichtlich der Spurenelementgehalte.

Wird für die vorliegenden Versuchsergebnisse der LER berechnet, so ergibt sich in beiden Versuchsjahren und an beiden Standorten ein LER von genau eins (Daten nicht dargestellt). Zustande kommt dies, da der Amarant gerade einmal einen Anteil von 2-8 \% am GesamtTrockenmasseertrag des Gemenges ausmacht und damit deutlich unterrepräsentiert ist. Der Bestand unterscheidet sich daher nur unwesentlich von der Mais-Reinsaat. Für die Berechnung des LER bedeutet dies, dass vom Relativertrag des Maises ein starker Einfluss ausgeht. Ob durch den Gemengeanbau aus Amarant und Mais, wie es in dieser Untersuchung technisch durchgeführt wurde, tatsächlich eine Qualitätsverbesserung und eine Spurenelementanreicherung durch den Amarant möglich wäre, kann bezweifelt werden. Eine Schwierigkeit bei der Etablierung des Gemenges bestand in den nur geringen Feldaufgängen des Amarants und einer unterschiedlichen Entwicklung der Amarantpflanzen aufgrund verzögerten Auflaufens (Daten nicht dargestellt). Möglicherweise hat hier der Mais das, aufgrund ausgebliebener Niederschläge zur Aussaat (Abbildung 2), wenige zur Verfügung stehende Keimwasser für sich beansprucht. Auch der mit $15-18^{\circ} \mathrm{C}$ (Kauffman \& Weber 1990) höhere Anspruch an die Bodentemperatur zur Keimung im Vergleich zu Mais mit 8-10 ${ }^{\circ} \mathrm{C}$ (Herrmann et al. 2011) kann eine Rolle gespielt haben. Zu dem geringen Feldaufgang und der daraus resultierenden geringen Anzahl an Amarantpflanzen je Flächeneinheit kam hinzu, dass die vorhandenen Amarantpflanzen vom Mais überwachsen wurden und sich nur schlecht etablieren konnten, wie in Abbildung 5 ersichtlich. Förster et al. (2019) variierten bei ihrer Untersuchung des Gemengeanbaus von Amarant und Mais den Saattermin des Maises. Wurden Amarant und Mais zusammen ausgesät, dominierte der Mais den Bestand, wurde der Mais 13 Tage nach dem Amarant gesät, dominierte hingegen der Amarant den Bestand. Dies spiegelte sich auch in den Trockenmasseerträgen wider. So unterschied sich bei den Autoren das Gemenge aus Amarant und früh gesätem Mais nicht von der Mais-Reinsaat und das Gemenge aus Amarant und spät gesätem Mais nicht von der Amarant-Reinsaat. Daran und an den Ergebnissen der vorliegenden Untersuchung wird deutlich, dass das Gemenge-Anbausystem aus Amarant und 
Mais noch weiterentwickelt werden muss. Ein Ansatz wäre der Streifenanbau von Amarant und Mais. Damit wären wahrscheinlich eine Reduzierung der interspezifischen Konkurrenz und eine geringere Beschattung des Amarants durch den Mais möglich. lqbal et al. (2019) sprechen aufgrund der ungleichen Ressourcennutzung, insbesondere der Ressource Licht, von einem ungünstigen Gemenge-Anbausystem, wenn Mais und Sojabohnen (Glycine max) in alternierenden Reihen angebaut werden. Im Streifenanbau dagegen war eine hohe Lichtaufnahme für beide Gemengepartner gegeben und die Sojabohnen waren weniger Stress durch die Beschattung durch den Mais ausgesetzt.

Im Gegensatz z.B. zu Amarant ist Mais züchterisch, unter Nutzung eines leistungsfähigen Hybridsystems (Becker 2011), eine sehr gut bearbeitete Feldfrucht. So ist es auch zu erwarten, dass Mais einen im Vergleich zu Amarant und anderen in diese Untersuchung eingehende Varianten, wesentlich höheren Ertrag erzielen kann. Mit einer Siloreifezahl von S220 gehört die in der Mais-Reinsaat und im Gemenge aus Amarant und Mais angebaute Maissorte Amadeo zur frühen Reifegruppe. Diese Auswahl erfolgte, um insbesondere im Gemenge aus Amarant und Mais, den zu erwartenden geringen TS-Gehalt des Amarants (Eberl et al. 2014) mit einem hohen TS-Gehalt der frühen Maissorte begegnen und aufheben zu können. Möglicherweise wären bei der Mais-Reinsaat und sicherlich auch bei Mais im Gemenge mit Amarant im untersuchten Anbausystem noch höhere Erträge zu erreichen gewesen, wenn eine Sorte mit höherer Siloreifezahl genutzt worden wäre. Vor allem im zweiten Versuchsjahr wäre zumindest am Standort Reinshof, an dem der Mais bei einem Erntetermin Mitte September bereits $35 \%$ TS (Tabelle 7) erreicht hatte, eine längere Vegetationszeit und damit eine längere Zeit der Assimilateinlagerung nutzbar gewesen. Dies hätte sich wahrscheinlich auch in einem höheren Wert für den Teilindex Trockenmasseertrag niedergeschlagen.

Aufgrund hoher Trockenmasseerträge fällt die Bewertung des Deutschen Weidelgrases mittels des Teilindex Trockenmasseertrag insbesondere am Standort Schoningen sehr positiv aus - besser sogar im Vergleich zu Mais als Hauptfrucht und den meisten Varianten des Zweikulturnutzungssystems (Abbildung 4, Abbildung 7, Tabelle 20).

Hohe Trockenmasseerträge waren beim Deutschen Weidelgras am Standort Reinshof zu erwarten, denn wie bei anderen Kulturpflanzen auch, ermöglichen tiefgründige Böden mit guter Wasserversorgung hohe Erträge bei Ackerfuttergräsern (Lütke Entrup 2011). Die Standortanforderung einer guten und ausgeglichenen Wasserversorgung war am Standort Schoningen in der Vegetationszeit des zweiten Versuchsjahres hingegen eher weniger gegeben. Zwar waren auch am Standort Reinshof bis auf den Monat Juni die Frühjahrs- und Sommermonate des zweiten Versuchsjahres von Trockenheit geprägt, jedoch war hier über die Vegetationszeit hinweg zumindest in den tieferen Bodenschichten mehr pflanzenverfügbares Wasser vorhanden als am Standort Schoningen (Abbildung 20, Abbildung 21). Dass das Deutsche Weidelgras am Standort Schoningen (198 dt TM ha ${ }^{-1}$ ) den Trockenmasseertrag des Standorts Reinshof (189 dt TM ha-1) im zweiten Versuchsjahr mit etwa $10 \mathrm{dt} \mathrm{TM} \mathrm{ha-1} \mathrm{dennoch} \mathrm{überstieg,} \mathrm{ist}$ daher überraschend. Wahrscheinlich spielen hier die Winterniederschläge, die am Standort Schoningen höher waren als am Standort Reinshof, eine Rolle.

Mit über $100 \mathrm{dt} \mathrm{TM} \mathrm{ha-1} \mathrm{im} \mathrm{ersten} \mathrm{bzw.} 90 \mathrm{dt} \mathrm{TM} \mathrm{ha}^{-1}$ im zweiten Versuchsjahr am Standort Reinshof und ebenfalls etwa $100 \mathrm{dt} \mathrm{TM} \mathrm{ha}^{-1}$ im zweiten Versuchsjahr am Standort Schoningen 
trug der erste Schnitt Anfang Juni erheblich zum Gesamttrockenmasseertrag des dreischnittigen Deutschen Weidelgrases bei. Damit zog es besonders am Standort Schoningen z.T. gleich mit den Trockenmasseerträgen der Erstfrüchte des Zweikulturnutzungssystems bzw. überstieg diese sogar. Das spricht für eine sehr effektive Nutzung der verfügbaren Ressourcen, so auch der Winterfeuchtigkeit durch das Deutsche Weidelgras. Günstig auf die Ertragsbildung wirkte sich aber sicherlich auch der an beiden Standorten im Vergleich zum langjährigen Mittel milde und überwiegend auch feuchte Herbst und Winter der beiden Versuchsjahre aus. Gerade dies ist laut Karsten \& McAdam (2001) bei Deutschem Weidelgras für die Einlagerung von Kohlenhydraten als Reservestoffe für den Austrieb in der darauffolgenden Vegetationsperiode sehr wichtig.

Im Rahmen des Anbaus von Winterzwischenfrüchten fand Menke (2011) beim Schnitt des Deutschen Weidelgrases im Mai mit $78 \mathrm{dt} \mathrm{TM} \mathrm{ha}{ }^{-1}$ im ersten und $42 \mathrm{dt} \mathrm{TM} \mathrm{ha}^{-1}$ im zweiten Versuchsjahr z.T. deutlich geringere Trockenmasseerträge im Vergleich zum ersten Schnitt des vorliegenden Versuchs. Anders als im vorliegenden Versuch, wurde das Weidelgras bei diesem Autor allerdings früher geerntet und im Frühjahr nicht mit Stickstoff gedüngt. Unterschiedliche Termine beim ersten Schnitt des Deutschen Weidelgrases lagen zwischen den beiden Standorten des vorliegenden Versuchs ebenfalls vor, wobei am Standort Reinshof sieben Tage früher als am Standort Schoningen geerntet wurde. Damit stand am Standort Reinshof eine kürzere Vegetationszeit für den ersten Aufwuchs zur Verfügung. Unter Annahme der von Menke (2011) ermittelten Wachstumsraten des Deutschen Weidelgrases von 8,5 g TM $\mathrm{m}^{-2} \mathrm{~d}^{-1}$ (Zeitraum Mitte April bis Ende zweite Dekade im Mai) käme bei einer sieben Tage späteren Ernte ein Trockenmasseertrag von etwa 6 dt TM ha-1 hinzu, was einen Teil des Ertragsunterschieds beim ersten Schnitt im zweiten Versuchsjahr zwischen den Standorten erklären könnte. Wie groß der Einfluss des Schnitttermins auf den Trockenmasseertrag des ersten Schnitts sein kann, zeigten Tobi et al. (2011). So hatte bei diesen Autoren ein später, an ein Drei-Schnitt-System angepasster Termin für den ersten Schnitt einen doppelt so hohen Trockenmasseertrag im Vergleich zu einem frühen, an ein Fünf-Schnitt-System angepasster Termin für den ersten Schnitt.

Im zweiten Versuchsjahr konnten am Standort Reinshof die Varianten des Zweikulturnutzungssystems und der Mais die Standortgegebenheiten besser nutzen um höhere Erträge zu erreichen als das Deutsche Weidelgras. Daraus ergab sich ein sehr hoher Mittelwert für die Trockenmasseerträge aller Varianten. So kommt es dazu, dass trotz eines immer noch hohen Trockenmasseertrags von etwa $190 \mathrm{dt} \mathrm{TM} \mathrm{ha}^{-1}$ diese Variante beim Teilindex Trockenmasseertrag in einem negativen Wert resultierte. Im Vergleich mit Untersuchungen anderer Autoren wurden in der vorliegenden Arbeit sehr hohe Trockenmasseerträge beim Deutschen Weidelgras erzielt. Techow et al. (2011) fanden Trockenmasseerträge in einer Vegetationsperiode von 170 in einem und $140 \mathrm{dt} \mathrm{TM} \mathrm{ha}^{-1}$ im anderen Jahr der Untersuchung, Sieling et al. (2013) ermittelten $139 \mathrm{dt} \mathrm{TM} \mathrm{ha-1}$. Zu berücksichtigen ist aber, in welchem Nutzungsjahr des Deutschen Weidelgrases in Dauerkultur die Ergebnisse ermittelt wurden. Tobi et al. (2011) erklären Ertragsrückgänge beim Deutschen Weidelgras bereits im dritten Nutzungsjahr unter anderem mit dem Alter des Bestandes. Deutlich höhere Trockenmasseerträge im Vergleich zur vorliegenden Untersuchung wurden mit $223 \mathrm{dt} \mathrm{TM} \mathrm{ha}^{-1}$ im ersten Hauptnutzungsjahr bei einer Mischung aus Welschem Weidelgras, Bastardweidelgras und Deutschem Weidelgras im Rahmen des EVA-Verbundvorhabens gefunden (Eckner et al. 2018). 
Die Werte für den Teilindex Trockenmasseertrag der Durchwachsenen Silphie machen deutlich, dass die Trockenmasseerträge dieser Variante an beiden Standorten relativ genau die mittleren Erträge aller Varianten abbilden (Tabelle 19, Tabelle 20). Da die Durchwachsene Silphie eine Dauerkultur mit etwa 15 Jahren Nutzungszeit ist und im Jahr der Pflanzung bzw. Aussaat nur eine Rosette bildet (Gansberger et al. 2015), liegen hier nur einjährige Daten vor. Mit $220 \mathrm{dt} \mathrm{TM} \mathrm{ha}^{-1}$ am Gunststandort Reinshof (Abbildung 4) bildete sie hier bereits im ersten Jahr der Beerntung einen erstaunlich hohen Trockenmasseertrag, der dem des Maises als Hauptfrucht nicht wesentlich nachstand. Trockenmasseerträge bei der Durchwachsenen Silphie in dieser Größenordnung fanden mit 160-222 dt TM ha ${ }^{-1}$ auch Hartmann \& Lunenberg (2016) bei einem vierjährigen Feldversuch auf einem Hochertragsstandort in Bayern. In Untersuchungen zum mehrjährigen Anbau der Durchwachsenen Silphie in Thüringen, zeigten sich in der Mehrzahl der acht Versuchsjahre höhere Trockenmasseerträge bei der Durchwachsenen Silphie (im Mittel über acht Jahre und zwei Standorte $217 \mathrm{dt} \mathrm{TM} \mathrm{ha}^{-1}$ ) im Vergleich zu Silomais (im Mittel über acht Jahre und zwei Standorte $190 \mathrm{dt} \mathrm{TM} \mathrm{ha}{ }^{-1}$; Biertümpfel \& Conrad 2013). Einen eindeutigen Trend zu einem etwaigen Anstieg der Trockenmasseerträge mit zunehmender Nutzungsdauer der Durchwachsenen Silphie ließen diese Untersuchungen nicht erkennen. Anders Siaudinis et al. (2017), die einen Anstieg der Erträge mit fortschreitender Nutzungsdauer beobachteten, verbunden mit einer deutlichen Zunahme der Anzahl Triebe je Pflanze.

Mit 150 dt TM ha-1 wurde am Standort Schoningen (Abbildung 7) deutlich weniger Biomasse bei der Durchwachsenen Silphie geerntet als am Standort Reinshof. Unterschiedliche Erträge auf diesen beiden Standorten waren aufgrund der Standortgegebenheiten zu erwarten. Während jedoch der Maisertrag am Standort Schoningen etwa $20 \%$ unter dem am Standort Reinshof lag und der Trockenmasseertrag des Deutschen Weidelgrases am Standort Schoningen sogar höher war als am Standort Reinshof, war der Ertragsunterschied zwischen den Standorten mit über $30 \%$ bei der Durchwachsenen Silphie erheblich größer. Bestandsbeobachtungen gaben am Standort Schoningen Hinweise auf bereits früh in der Vegetationszeit eingetretenen Trockenstress bei den Silphiepflanzen. Nach zunächst guter Bestandesentwicklung wurden massive Welkeerscheinungen an den Blättern sichtbar, noch bevor der Bestand in die Vollblüte kam (Abbildung 9). Auch der hohe TS-Gehalt von $35 \%$ bei relativ früher Ernte (25. August 2016) weist auf die trockene Witterung vor der Ernte und die weit vorangeschrittene Seneszenz der Pflanzen hin. Im Vergleich dazu hatte am Standort Reinshof der Aufwuchs der Durchwachsenen Silphie etwa eine Woche später gerade einmal $22 \%$ Trockensubstanz. Bei einer Untersuchung zum Einfluss des Entwicklungsstadiums auf den Trockenmasseertrag konnte Pichard (2012) einen Anstieg bis zum Stadium der Samenbildung feststellen. Auch Biertümpfel \& Conrad (2013) konnten in Abhängigkeit vom Standort ein optimales Zeitfenster für die Ernte der Durchwachsenen Silphie ausmachen bis zu dem der Trockenmasseertrag auf ein Maximum stieg und danach wieder abfiel. Bei späteren Erntezeitpunkten nahm er durch das Einsetzen von Blattverlusten ab. In der vorliegenden Untersuchung am Standort Schoningen kann davon ausgegangen werden, dass die Biomassezuwächse bei der Durchwachsenen Silphie in der Zeit nach Blühbeginn durch die Trockenheitsschäden eher gering waren. Auch ein bereits eingetretener Blattverlust kann hier zusätzlich den Trockenmasseertrag negativ beeinflusst haben. Durch ihre morphologische Eigenschaft, am Stängel verwachsener Blattpaare, die Becher formen, in denen die Pflanzen Regen- und Tauwasser auffangen können, kann bei der Durchwachsenen Silphie eine gewisse Trockenheitstoleranz angenommen werden. Untersuchungen von Schoo et al. (2017) zeigen jedoch, dass der Beitrag des in den 
Bechern aufgefangenen Tauwassers zur Biomasseproduktion der Durchwachsene Silphie nur gering war. Im Gegenteil, der trockenstressbedingte Ertragsrückgang war bei der Durchwachsenen Silphie weitaus höher als bei Mais und Luzernegras. Der Empfehlung der Autoren, die Durchwachsene Silphie nur an Standorten mit guter Wasserversorgung anzubauen, kann anhand der eigenen Ergebnisse beigepflichtet werden.

Signifikant verschieden zu den an diesem Standort erreichten Trockenmasseerträgen von Mais und dem Gemenge aus Amarant und Mais waren die Trockenmasseerträge der Durchwachsenen Silphie trotz alledem nicht. Wie auch der Teilindexwert nahe null zeigt, ordnet sich die Durchwachsene Silphie trotz des vergleichsweise geringen Ertrags in das insgesamt geringere Ertragsniveau am Standort Schoningen ein.

Wesentlich geringer als die vorgestellten Varianten und deutlich negativ bewertet beim Teilindex Trockenmasseertrag ist das Ertragspotenzial von Amarant in Reinsaat, der ein- und mehrjährigen Blühmischung sowie der Sommerackerbohnen (Abbildung 4, Abbildung 7, Tabelle 19, Tabelle 20).

Der Amarant in Reinsaat lieferte am Standort Reinshof mit etwa 125 dt TM ha-1 einen im Vergleich mit einigen anderen Varianten an diesem Standort geringen Trockenmasseertrag. Insbesondere mit den bekannten Feldfrüchten und Anbausystemen im Rahmen des Energiepflanzenanbaus (Mais, Zweikulturnutzungssysteme und Deutsches Weidelgras) kann der Amarant ertraglich nicht mithalten. Am Standort Schoningen waren die Trockenmasseerträge des Amarants mit etwa 100 dt TM ha ${ }^{-1}$ zwar deutlich höher als die der Blühmischungen, aber ebenfalls deutlich geringer als die der bekannten Energiepflanzenvarianten. Angebaut wurde die Amarantsorte Bärnkrafft, als einzige im Versuchszeitraum im deutschen Sortenregister eingetragene Amarantsorte (Bundessortenamt 2015). Diese Sorte wird eigentlich zur Kornnutzung angebaut (Eberl et al. 2014). Die Annahme, eine Sorte zur Biomassenutzung, wie es bei der aus Russland stammenden Futternutzungssorte Pastewny der Fall ist (Kaul et al. 1996), würde höhere Trockenmasseerträge hervorbringen als eine Kornnutzungssorte, bestätigte sich im eigenen Zusatzversuch im Versuchsjahr 2016 jedoch nicht.

In dem am Technologie- und Förderzentrum im Kompetenzzentrum für Nachwachsende Rohstoffe durchgeführten Amarant-Sortenscreening sollten Sorten und Linien mit besonderer Eignung für die Nutzung als Energiepflanze für die Biogaserzeugung identifiziert und selektiert werden (Eberl et al. 2014). Die dabei gefundenen, für die Nutzung als Energiepflanze vielversprechendsten der 150 im Sortenscreening untersuchten, weltweit zusammengetragenen Amarantsorten und -linien wurden in pflanzenbaulichen Feldversuchen weiter untersucht (Förster et al. 2019). Die Auswahlkriterien waren u.a. Trockenmasseertrag, Trockensubstanzgehalt, frühe Abreife, Standfestigkeit und ein möglichst hoher Spurenelementgehalt. Bei 35 der 40 getesteten, vielversprechenden Amarantsorten und -linien fanden die Autoren im Versuchsjahr 2018 Trockenmasseerträge von mindestens $150 \mathrm{dt} \mathrm{TM} \mathrm{ha-1}$ - einige davon reichten sogar bis knapp an 190 dt TM ha-1 heran. Die Ergebnisse von Förster et al. (2019) weisen damit auf ein recht hohes Ertragspotenzial beim Amarant hin. Die in der vorliegenden Untersuchung betrachtete Sorte Bärnkrafft ging bei diesen Autoren allerdings nicht in die Ertragsermittlung ein, was darauf hindeutet, dass diese, nachdem sie im Sortenscreening (Eberl et al. 2014) getestet wurde, als nicht vielversprechend zur Nutzung als Energiepflanze nach den 
angewandten Kriterien eingestuft wurde. Die Sorte Pastewny dagegen ging als vielversprechende Sorte in die Untersuchung von Förster et al. (2019) ein und erzielte mit $170 \mathrm{dt}$ TM ha $^{-1}$ einen deutlich höheren Trockenmasseertrag als in der vorliegenden Untersuchung mit 133 dt TM ha-1 ${ }^{-1}$ Die hohen Temperaturen des Jahres 2018 am Standort Straubing kamen, so Förster et al. (2019), dem Amarant als $\mathrm{C}_{4}$-Pflanzen mit hohem Wärmebedarf besonders entgegen. Mit den Trockenmasseerträgen der vorliegenden Untersuchung vergleichbare Erträge fanden von Cossel et al. (2017) mit $130 \mathrm{dt} \mathrm{TM} \mathrm{ha}^{-1}$ im Mittel über die Versuchsjahre bei einem in der Entwicklung zur Biomassenutzung befindlichen Amarant-Genotyp. Jedoch zeigten die Autoren auch Jahreseffekte mit Trockenmasseerträgen von 150 und $100 \mathrm{dt} \mathrm{TM} \mathrm{ha-1} \mathrm{in} \mathrm{den} \mathrm{beiden} \mathrm{Ver-}$ suchsjahren auf, wobei der geringe Ertrag auf die Trockenheit im entsprechenden Versuchsjahr zurückzuführen war. Mais reagierte bei diesen Autoren allerdings noch stärker auf die Trockenheit.

Amarant wird eine gewisse Toleranz gegenüber Trockenheit zugeschrieben was u.a. auf den $\mathrm{C}_{4}$-Photosythesestoffwechsel und die lange Pfahlwurzel zurückgeht (Johnson \& Henderson 2002). So wurde bei Untersuchungen in China herausgefunden, dass der Wasserbedarf für den Anbau von Korn-Amarant 42-47 \% dem des Weizenanbaus und 51-62 \% dem des Maisanbaus entspricht. Die Trockentoleranz ist jedoch in späteren Entwicklungsstadien ausgeprägter als in frühen, vielmehr bedarf es gerade nach der Aussaat genug Feuchtigkeit für einen sicheren Feldaufgang (Kauffman \& Weber 1990). Das hohe Niederschlagsdefizit in den Sommermonaten des zweiten Versuchsjahres (Juli-September 2016, Abbildung 2) der vorliegenden Untersuchung im Vergleich zum langjährigen Mittel aber auch im Vergleich zum ersten Versuchsjahr sowie die geringen Mengen pflanzenverfügbaren Wassers im Verlauf der Vegetationszeit des zweiten Versuchsjahres im Boden (Abbildung 20, Abbildung 21), schlugen sich nicht in unterschiedlichen Amaranterträgen zwischen den Versuchsjahren nieder. Das Gleiche gilt allerdings auch für die anderen untersuchten Varianten (Abbildung 4, Abbildung 7). Daher kann eine höhere Trockenheitstoleranz des Amarants gegenüber den anderen Feldfrüchten hier nicht bestätigt werden. Ein nur geringer Feldaufgang und stark verzögert auflaufende Amarantpflanzen (Daten nicht dargestellt) konnten, bedingt durch die Trockenheit im Mai und z.T. im Juni der beiden Versuchsjahre jedoch beobachtet werden. Ob eine geringere als angestrebte Bestandesdichte auch ertragswirksam war, kann nur schwer abgeschätzt werden. Ergebnisse von Förster et al. (2019) weisen auf ein großes Kompensationsvermögen bei geringer Bestandesdichte der Amarantpflanzen hin.

Der Amarant könnte bei Annahme günstiger Auflaufbedingungen auch in Zweitfruchtstellung stehen, um in Verbindung mit einer Erstfrucht einen höheren Trockenmasseertrag in einem solchen Anbausystem zu generieren. Dies könnte, ähnlich den Varianten des hier untersuchten Zweikulturnutzungssystems in Relation zu Mais als Hauptfrucht, ebenfalls zu einer günstigeren Bewertung im Teilindex Trockenmasseertrag führen. Die angesprochenen notwendigen hohen Bodentemperaturen zur Keimung des Amarants sprechen ohnehin für einen vergleichsweise späten Saattermin.

In die Untersuchungen ging die einjährige Blühmischung BG80 mit 12 einjährigen Arten und die mehrjährige Blühmischung BG70 mit 25 z.T. einjährigen, zweijährigen und ausdauernden Arten ein. Das Anbaukonzept der mehrjährigen Blühmischung sieht vor, dass im ersten der fünf Nutzungsjahre, also im Jahr der Aussaat, die einjährigen Arten den Bestand bilden und Biomasse liefern. Die zwei- und mehrjährigen Arten entwickeln sich im ersten Standjahr unter 
den einjährigen Arten, um dann ab dem zweiten Standjahr den Bestand zu dominieren (Vollrath 2012). So erfolgt in jedem Jahr eine Ernte. Dies ist ein wesentlicher Unterschied zur Durchwachsenen Silphie, bei der, zumindest bei dem in der vorliegenden Untersuchung angewandten Etablierungsverfahren über Pflanzung von Jungpflanzen, das Jahr nach der Etablierung das erste Erntejahr war.

Am Standort Reinshof entwickelten sich beide Blühmischungen in beiden Versuchsjahren gut. So erbrachte die einjährige Blühmischung hier in beiden Versuchsjahren einen nahezu identischen Trockenmasseertrag von etwa $135 \mathrm{dt} \mathrm{TM} \mathrm{ha-1} \mathrm{(Abbildung} \mathrm{4).} \mathrm{Die} \mathrm{mehrjährige} \mathrm{Blühmi-}$ schung fiel besonders im ersten Versuchsjahr, in dem bei dieser Variante ebenfalls die einjährigen Arten dominierten, auf unter $100 \mathrm{dt} \mathrm{TM} \mathrm{ha}^{-1} \mathrm{ab}$. Wie Vollrath (2012) beschreibt, bilden die einjährigen Arten der mehrjährigen Blühmischung im ersten Standjahr einen lockeren, lichtdurchlässigen Bestand um die Entwicklung der mehrjährigen Arten zu gewährleisten. Dies konnte auch in den vorliegenden Untersuchungen festgestellt werden. Im Vergleich zur einjährigen Blühmischung, die einen sehr üppigen Bestand bildete, war der Bestand im ersten Versuchsjahr bei der mehrjährigen Blühmischung hier eher dünn. Von der, in der einjährigen Blühmischung den Bestand dominierenden, üppig wirkenden Art, Malva sylvestris, war der Anteil am Saatgut in der mehrjährigen Blühmischung auch deutlich geringer. In einer Untersuchung zu verschiedenen Verfahren der Bestandsgründung der mehrjährigen Blühmischung stellten von Cossel et al. (2019) fest, dass bei Etablierung der mehrjährigen Blühmischung als Untersaat in Mais, das erste, ertragsschwache Jahr bei der mehrjährigen Blühmischung umgangen werden kann. Dies würde auch beim hier vorgestellten Vorgehen zu einer deutlichen Verbesserung in der Bewertung der mehrjährigen Blühmischung führen.

Im zweiten Versuchsjahr zeigte sich ein signifikant höherer Trockenmasseertrag der mehrjährigen Blühmischung am Standort Reinshof im Vergleich zum ersten Versuchsjahr. Die dominierende Art war die Schwarze Flockenblume (Centaurea nigra). Gleiches beobachteten auch von Cossel \& Lewandowski (2016), wobei die Schwarze Flockenblume auch in den Folgejahren der fünfjährigen Untersuchung dieser Autoren einen großen Anteil am Aufwuchs ausmachte; ab dem dritten Versuchsjahr überwog dann der Rainfarn (Tanacetum vulgare). Insgesamt fanden die Autoren einen starken Rückgang der Artenanzahl ab dem dritten Standjahr der Blühmischung auf drei bis vier Arten. Mit etwa $130 \mathrm{dt} \mathrm{TM} \mathrm{ha-1} \mathrm{im} \mathrm{zweiten} \mathrm{Versuchsjahr}$ reiht sich der Trockenmasseertrag des Standorts Reinshof gut in die von Vollrath (2012) gefundene Spanne an Trockenmasseerträgen im zweiten Standjahr von 60 bis $190 \mathrm{dt} \mathrm{TM}$ ha $^{-1}$ ein. Von Cossel \& Lewandowski (2016) fanden sogar Trockenmasseerträge von über $220 \mathrm{dt}$ $\mathrm{TM} \mathrm{ha}^{-1}$ im fünften Standjahr an einem der drei Versuchsstandorte. An den anderen Standorten dieser Autoren konnten keine so hohen Erträge erzielt werden. Das zeigt, dass die mehrjährige Blühmischung ein hohes Ertragspotenzial hat, dieses aber auch nicht immer erreicht werden kann. Vollrath (2012) weist darauf hin, dass es sich bei dem Anbausystem der mehrjährigen Blühmischungen zur Biogaserzeugung um ein vergleichsweise neues Konzept handele, das ertraglich noch nicht mit züchterisch stark bearbeiteten und pflanzenbaulich gut verstandenen Feldfrüchten mithalten könne.

Am Standort Schoningen waren die Trockenmasseerträge beider Blühmischungen in beiden Versuchsjahren weitaus geringer als am Standort Reinshof. Die Parzellen der ein- und mehrjährigen Blühmischung waren hier stark verunkrautet - im zweiten Versuchsjahr noch massiver als im ersten. Dies spiegelte sich auch im Wert für den Teilindex Trockenmasseertrag 
wider, der hier bei der einjährigen Blühmischung mit deutlich Abstand den größten negativen Betrag innerhalb dieses Teilindex hatte. Die Leitunkräuter waren Geruchlose Kamille (Matricaria inodora) und Purpurrote Taubnessel (Lamium purpureum; Abbildung 8). Da bei den Blühmischungen, anders als bei den meisten Ackerbaukulturen, bei denen Herbizidanwendungen problemlos möglich sind, eine chemische Unkrautkontrolle nicht und eine mechanische Unkrautkontrolle nur schwer möglich ist, wurde, wenn auch nicht praxisüblich bzw. praxistauglich, mit mäßigem Erfolg von Hand gejätet. Von einer Ertragsreduktion aufgrund der starken Unterdrückung der Blühpflanzen durch das Unkraut kann ausgegangen werden. Dies bedeutet, dass die Standorteignung einer Blühmischungen auch mit dem zu erwartenden Unkrautaufkommen zusammenhängt.

Die Sommerackerbohnen entwickelten sich an beiden Standorten in beiden Versuchsjahren gut, gehörten jedoch zu den Varianten mit den geringsten Trockenmasseerträgen (Abbildung 4, Abbildung 7). Am Standort Reinshof waren sie sogar die, mit Blick auf die Trockenmasseerträge, ungünstigste Variante, wie der Wert für den Teilindex Trockenmasseertrag zeigt (Tabelle 19). Im ersten Versuchsjahr lag der Trockenmasseertrag der Sommerackerbohnen an beiden Standorten mit etwa $20 \mathrm{dt} \mathrm{TM} \mathrm{ha}{ }^{-1}$ sogar deutlich unterhalb dem der Winterackerbohnen. Während den Winterackerbohnen aber noch eine Zweitfrucht und somit auch ein weiterer Ertragsbildner folgte, war dies bei den Sommerackerbohnen hier nicht der Fall. Mit den Sommerackerbohnen als Sommerhauptfrucht war es weder möglich einen konkurrenzfähigen Trockenmasseertrag zu erzielen noch die gesamte Vegetationszeit auszunutzen. Die Sommerackerbohnen wurden im vorliegenden Versuch im Juli geerntet. Dieser Erntetermin ergab sich aus einem Kompromiss zwischen den Trockensubstanzgehalten und dem beginnenden Blattfall bei den Ackerbohnenpflanzen. Zur effektiven Ausnutzung der Vegetationszeit und um die Bewertung dieser Variante im Teilindex Trockenmasseertrag zu verbessern, wäre zusätzlich zu den Sommerackerbohnen als Hauptfrucht der Anbau und die Nutzung einer Sommerzwischenfrucht denkbar. Für Saattermine im Juli kämen, abgesehen von verschiedenen Leguminosen in Reinsaat und Gemenge, z.B. Sonnenblumen, Brassicaceen oder Einjähriges Weidelgras infrage (Karpenstein-Machan 2005). Petersson et al. (2007) untersuchten die Möglichkeit, das Stroh von Sommerackerbohnen zur Vergärung in der Biogasanlage einzusetzen. Diese Art der Doppelnutzung ist von Mais bekannt (Fleschhut 2015). Das Korn wird dabei zur Fütterung eingesetzt und das Stroh in der Biogasanlage vergoren. Petersson et al. (2007) kamen zu dem Ergebnis, dass das Stroh der Ackerbohnen zur Methanerzeugung geeignet ist. Der im Biogasertragstest bestimmte spezifische Methanertrag war bei diesen Autoren mit $0,440 \mathrm{Nm}^{3} \mathrm{CH}_{4} \mathrm{~kg}^{-1}$ oTM sogar erstaunlich hoch. Vor dem Hintergrund der Spurenelementversorgung der Biogasanlage mithilfe von Sommerackerbohnen, wäre auch dies ein ausbaufähiger Ansatz.

Im zweiten Versuchsjahr war der Trockenmasseertrag der Sommerackerbohnen höher als im ersten Versuchsjahr, am Standort Schoningen sogar signifikant. Dies könnte auch mit Unterschieden im Management zwischen den Versuchsjahren zusammenhängen. So fand im zweiten Versuchsjahr an beiden Standorten die Aussaat früher statt als im ersten Versuchsjahr. Ein möglichst früher Saattermin ist bei Sommerackerbohnen für eine sichere Ertragsbildung vorzuziehen (Schäfer 2011). Zudem wurde im ersten Versuchsjahr ein Nachauflaufherbizid und im zweiten Versuchsjahr ein Vorauflaufherbizid eingesetzt. Ghosheh \& El-Shatnawi (2003) untersuchten die Wirkung verschiedener Herbizidstrategien auf die Unkrautkontrolle und die 
Verträglichkeit der Wirkstoffe bei Ackerbohnen. Die Autoren konnten zeigen, dass die Ackerbohnen eine signifikant geringere Gesamtbiomasse sowie einen signifikant geringeren Kornertrag bildeten, wenn sie mit einem Nachauflaufherbizid behandelt wurden im Vergleich zur Vorauflaufbehandlung. Beim Wirkstoff Bentazon, der sowohl bei Ghosheh \& El-Shatnawi (2003) als auch im hier vorliegenden Versuch im Nachauflauf eingesetzt wurde, sprechen die Autoren sogar von gehemmtem Wachstum der Pflanzen, was sich in einer signifikant geringeren Wuchshöhe im Vergleich mit anderen Wirkstoffen zeigte (Ghosheh \& El-Shatnawi 2003). Möglicherweise gab es eine solche Wuchsdepression und geringere Biomasseproduktion aufgrund des eingesetzten Nachauflaufherbizids im ersten Versuchsjahr des hier vorliegenden Versuchs ebenfalls.

\subsubsection{Teilindex Trockensubstanzgehalt}

Ein wichtiges Kriterium für die Eignung einer Feldfrucht als Energiepflanze für die Biogaserzeugung ist der Trockensubstanzgehalt (Förster et al. 2019). Für eine erfolgreiche Silierung werden Trockensubstanzgehalte im Bereich von 28-35\% angestrebt. Bei Trockensubstanzgehalten unterhalb von $28 \%$ kommt es zu Sickersaftverlusten, oberhalb von $35 \%$ zu Schwierigkeiten bei der, zur Verhinderung der Erwärmung, nötigen Verdichtung des Silostapels (Herrmann \& Rath 2012). Sickersaftverluste aufgrund geringer TS-Gehalte bedeuten auch Masseverluste und führen damit wiederum zu einer Abnahme des Methanertrags (Weiland 2010).

In der vorliegenden Untersuchung wies der Teilindex TS-Gehalt auf Schwächen hinsichtlich der Erreichung ausreichend hoher TS-Gehalte bei einigen Varianten hin. An beiden Versuchsstandorten war im Mittel über beide Versuchsjahre bei mehr als der Hälfte der Varianten der Indexwert hier negativ (Tabelle 19, Tabelle 20). Allerdings gab es auch Unterschiede zwischen den Versuchsjahren. Nicht alle Varianten zeigten in beiden Versuchsjahren das gleiche Bild. Bei der Berechnung der Teilindexwerte für den Teilindex TS-Gehalt wurden, wie bei der Berechnung der überwiegenden Zahl der Teilindizes, die einzelnen Varianten ins Verhältnis gesetzt zum Mittelwert aller Varianten. Diese lagen an den Standorten Reinshof und Schoningen bei 24 bzw. $27 \%$ und 23 bzw. $30 \%$ im ersten bzw. zweiten Versuchsjahr. Werden diese Werte eingeordnet in den Bereich der Trockensubstanzgehalte, die für eine verlustfreie Silierung (28$35 \%$ ) nötig sind, so wird deutlich, dass sie am Standort Reinshof in beiden Versuchsjahren und am Standort Schoningen zumindest im ersten Versuchsjahr unterhalb des angestrebten Bereichs liegen. Bei der vorgenommenen Indexberechnung werden nur Varianten mit TS-Gehalten unterhalb des Mittelwerts mit negativen Indexwerten versehen. Ist also der mittlere TSGehalt der Varianten selbst gering, so werden dennoch nur Varianten mit noch geringeren TSGehalten negativ bewertet. Andere Varianten, die ebenfalls unterhalb der Schwelle von $28 \%$ liegen, wiederum nicht. Das bedeutet, dass ein positiver Indexwert nicht unbedingt gleichbedeutend ist mit optimaler Silierfähigkeit. Die Möglichkeit, verlustfrei zu silieren wird in diesen Fällen eher überschätzt. Auf der anderen Seite gab es auch Varianten mit TS-Gehalten größer $35 \%$, also ebenfalls außerhalb des angestrebten Bereichs. Die angewandte Indexberechnung bewertet jedoch umso besser, je höher die Werte sind. Anstatt Varianten mit Trockensubstanzgehalten von über $35 \%$ negativ zu bewerten, da die Biomasse möglicherweise nicht optimal verdichtet werden kann, werden diese Varianten dadurch beim Teilindex TS-Gehalt sogar belohnt. Eine Möglichkeit diesem Problem zu begegnen wäre, nicht den Mittelwert der TS-Gehalte aller Varianten für die Teilindexberechnung zu verwenden, sondern den Bereich mit Trockensubstanzgehalten für verlustfreie Silierung. TS-Gehalte von weniger als $28 \%$ und mehr 
als $35 \%$ würden dabei negativ bewertet. Ziel der vorliegenden Arbeit war es jedoch, die Pflanzenvarianten zu vergleichen und ohne feste Zielwerte zu bewerten.

Bei den Varianten des Zweikulturnutzungssystems wurden die Teilindexwerte unter Annahme einer Mischung der beiden Komponenten, Winterzwischenfrucht und Zweitfrucht und Berücksichtigung der jeweiligen Anteile, berechnet. Trotz dieser Berechnungsweise und zumindest im zweiten Versuchsjahr optimaler TS-Gehalte der Zweitfrucht Mais, waren die Indexwerte für den Teilindex TS-Gehalt bei diesen Varianten überwiegend negativ. Der Erntetermin der Erstfrüchte des Zweikulturnutzungssystems wurde so gewählt, dass die Erstfrüchte möglichst weit in ihrer Entwicklung vorangeschritten waren um sowohl hohe Trockenmasseerträge als auch hohe Trockensubstanzgehalte zu erreichen. Auf der anderen Seite sollte auch dem Mais als Zweitfrucht ausreichend Vegetationszeit zur Verfügung stehen, was einen möglichst frühen Saattermin erfordert. Bei den getreidebetonten Erstfrüchten führte dieses Vorgehen z.T. zu recht hohen TS-Gehalten: der Wickroggen erreichte am Standort Reinshof und die Wintertriticale am Standort Schoningen $28 \%$ TS und waren damit verlustfrei silierbar. Jedoch war dies nicht in beiden Versuchsjahren an beiden Standorten der Fall. Am Standort Reinshof könnten die höheren TS-Gehalte bei den Erstfrüchten im zweiten Versuchsjahr im Vergleich zum ersten Versuchsjahr mit den trockenen Frühjahrsmonaten (März, April, Mai; Abbildung 2) des zweiten Versuchsjahres erklärt werden. Dies unterstützt die Befunde am Standort Schoningen jedoch nicht, da hier die TS-Gehalte im ersten, niederschlagsreicheren Versuchsjahr höher waren als im zweiten, niederschlagsärmeren. Auch wenn die Aussaat der Winterungen am Standort Schoningen im zweiten Versuchsjahr erst spät erfolgte, war hier auch die Ernte später, sodass von einer vergleichbaren Vegetationszeit bei den Versuchsjahren und Versuchsstandorten ausgegangen werden kann.

Dass hohe TS-Gehalte bei sogar noch früheren Ernteterminen erreicht werden können, zeigte Menke (2011) bei einer Untersuchung verschiedener Winterzwischenfrüchte im Zweikulturnutzungssystem mit Mais. Die untersuchten Getreidevarianten und auch der Wickroggen hatten bei diesem Autor bereits im Mai (21. Mai) TS-Gehalte von über $30 \%$. Im zweiten Versuchsjahr wurden solch hohe Werte bei Menke (2011) jedoch ebenfalls nicht erreicht, was hier bedingt durch einen späteren Saattermin im zweiten Versuchsjahr und der damit zusammenhängenden kürzeren Vegetationszeit als im ersten Versuchsjahr begründet sein könnte. Auch Graß et al. (2013) konnte bei Winterroggen und dem Gemenge aus Winterroggen und Wintererbsen als Erstfrüchte im Zweikulturnutzungssystem bei der Biomasseernte Anfang Juni TS-Gehalte von über $30 \%$ finden.

Deutlich geringer als bei den getreidebetonten Erstfrüchten und weit entfernt von den für eine verlustfreie Silierung angestrebten TS-Gehalten waren die TS-Gehalte bei den Winterackerbohnen mit Werten zwischen 13 und $16 \%$ (Tabelle 7, Tabelle 8). Ein späterer Erntetermin und damit einhergehendes vorangeschrittenes Entwicklungsstadium der Winterackerbohnen würde, wie im vorliegenden Versuch bei den Sommerackerbohnen beobachtet, nur zu einer unwesentlichen Verbesserung der TS-Gehalte führen. Die Sommerackerbohnen konnten selbst im Stadium der beginnenden Abreife nur höchstens $19 \%$ TS erreichen. Die Winterackerbohnen waren bei der Ernte in ihrer Entwicklung hingegen erst beim Ende der Blüte bis beginnender Hülsenentwicklung. Zudem ginge ein späterer Erntetermin auf Kosten der Zweitfrucht und der Erreichung hoher TS-Gehalte bei dieser Kultur. Zumindest ein Stück weit Abhilfe 
bei dem Problem sehr geringer TS-Gehalte kann der Anbau der Winterackerbohnen im Gemenge mit Wintertriticale schaffen, wie im vorliegenden Versuch mit TS-Gehalten von 19 bis $22 \%$ des Gemenges gezeigt werden konnte. Während sich dies auch in der Verbesserung des Teilindex TS-Gehalte des Gemenges im Vergleich zur Reinsaat widerspiegelte, war dies bei den Sommerackerbohnen nicht der Fall, im Gegenteil. Die Sommerackerbohnen hatten zwar etwas höhere TS-Gehalte als die Winterackerbohnen, jedoch wurde hier kein Partner für eine Mischsilage, wie bei den Winterackerbohnen der Mais, einbezogen. Daher wurden beim Teilindex TS-Gehalt die Sommerackerbohnen mit dem größten negativen Indexwert als ungünstigste Variante bewertet.

Es wird deutlich, dass die Konstruktion des Teilindex TS-Gehalt bei den Varianten des Zweikulturnutzungssystems unter Aufnahme der beiden Komponenten Erst- und Zweitfrucht vom Problem niedriger TS-Gehalte der Winterzwischenfrüchte ablenkt. Bei der Identifikation von Schwächen, was mit dem Index IrA und hier bei der Diskussion des Teilindex TS-Gehalt hinsichtlich der TS-Gehalte möglich ist, werden diese bei den Winterzwischenfrüchten daher eher unterschätzt. Die Suche nach Problemlösungen wird damit erschwert. Die Sommerackerbohnen hingegen gelangen aufgrund des Wertes für den Teilindex TS-Gehalt unmittelbar in den Fokus.

Borreani et al. (2009) konnten bei einer Untersuchung zur Siliereignung verschiedener Leguminosen als Ganzpflanzen zeigen, dass Ackerbohnen nach einer Anwelkphase und damit einhergehender Erhöhung der TS-Gehalte erfolgreich siliert werden können: Die Silage der angewelkten Ackerbohnen-Biomasse enthielt deutlich weniger Ammoniak und Buttersäure und höhere Gehalte an Milchsäure im Vergleich zur Silage des frischen Materials. Ein weiterer positiver Effekt des Anwelkens ist der Transport von weniger Wasser (Borreani et al. 2009). Das wäre u.a. auch für die ökonomische Bewertung der Ackerbohnen günstig, da dann auch die Transportkosten sinken würden. Franco et al. (2016) kommen in einer Literaturübersicht zu dem Ergebnis, dass Anwelken eine ökonomisch günstige und sehr effektive Methode ist, Biomasse erfolgreich zu silieren. Aber auch der Zusatz von Siliermitteln (Lactobacillus plantarum) konnte bei Borreani et al. (2009) einen unerwünschten hohen Buttersäuregehalt trotz geringer TS-Gehalte bei Ackerbohnen verhindern. Neben dem Anwelken der Biomasse wäre auch eine gemeinsame Silierung der Sommer- wie auch Winterackerbohnen mit Biomassen mit höheren TS-Gehalten denkbar. Auch Pahlow (2003) schätzt Leguminosen u.a. aufgrund hoher Proteingehalte als schlecht silierbar ein und rät z.B. die Zottel- und Saatwicken gemeinsam mit Getreide oder Gras zu silieren. Beim Erntetermin der Sommerackerbohnen würde sich möglicherweise der zweite Schnitt des Weidelgrases oder anderer Grünlandaufwüchse anbieten - vorausgesetzt, hier wären hohe TS-Gehalte möglich. Eine weitere Möglichkeit wäre es, feuchte Biomasse gemeinsam mit Stroh zu silieren. In einer Untersuchung zeigten Ugilt Larsen et al. (2017), dass Blätter von Zuckerrüben mit nur 13,5 \% TS zusammen mit Weizenstroh ohne Sickersaftverluste siliert werden konnten. Darüber hinaus konnten die Autoren synergistische Effekte bei der Methanproduktion der Mischsilage finden: Die Mischsilage aus Stroh und Zuckerrübenblatt hatte eine deutlich höhere Methanausbeute im Batch-Test als aus der Summe der unsilierten Einzelkomponenten zu erwarten war. Neben der Erreichung optimaler TS-Gehalte für die Konservierung führt die Silierung auch zu einem Aufschluss des sonst nur schwer abbaubaren Strohs (Laser 2019). Zu überlegen wäre daher, z.B. eine Mischsilage aus Sommerackerbohnen und dem etwa gleichzeitig anfallenden Gerstenstroh herzustellen. Dies könnte auf der einen Seite die Silierfähigkeit und damit auch die Anbauwürdigkeit 
der Ackerbohnen verbessern und auf der anderen Seite Stroh stärker als Koppelprodukt der Getreideproduktion nutzbar für die Biogaserzeugung machen.

Negative Werte beim Teilindex TS-Gehalt zeigten überwiegend auch die einjährige Blühmischung und der Amarant. Bei diesen Varianten besteht offenbar das Problem, dass bis zum Vegetationsende nur schwierig ausreichend hohe TS-Gehalte erreicht werden können. Im ersten Versuchsjahr wurde die Vegetationszeit durch den ersten Frost Anfang Oktober beendet wobei die Pflanzen abstarben. Zu diesem Zeitpunkt waren die angestrebten TS-Gehalte noch z.T. bei Weitem nicht erreicht. Auch wenn im zweiten Versuchsjahr kein Frostschaden zu verzeichnen war, waren die TS-Gehalte insbesondere am Standort Reinshof trotz eines vergleichsweise späten Erntetermins nicht höher. Am Standort Schoningen wurden im zweiten Versuchsjahr v.a. bei der einjährigen Blühmischung zwar höhere TS-Gehalte erreicht als im ersten, aber hier war, wie angesprochen, auch der Anteil an Unkraut höher, wodurch es möglicherweise zu einer Verfälschung des TS-Gehalts in der Biomasse gekommen sein könnte. Nur geringe Trockensubstanzgehalte beim Amarant mit Werten deutlich unterhalb von $28 \%$ fanden auch Eberl et al. (2014), von Cossel et al. (2017) und Förster et al. (2019). Wie diesen Arbeiten zu entnehmen ist, gibt es Bemühungen, Amarant weiterzuentwickeln und in der Praxis zu etablieren. Ein Zuchtziel für die Nutzung als Energiepflanze für die Biogaserzeugung muss daher die Erhöhung des TS-Gehalts sein. Dass dies bislang jedoch schwierig ist, zeigen Förster et al. (2019) bei der mehrjährigen Untersuchung von insgesamt 308 verschiedenen Amarantsorten und -linien mit TS-Gehalten in einer Spannbreite von 15,7 bis maximal 26,1\% TS bei der Ernte Ende September bis Anfang Oktober. Der maximal erreichte TS-Gehalt genügt also bei keiner, in diesem weit gefassten Sortenscreening untersuchten, Amarantsorte oder -linie den angestrebten $28 \%$.

Während die einjährige Blühmischung sowie das erste, von einjährigen Arten dominierte Standjahr der mehrjährigen Blühmischung nur unzureichend hohe TS-Gehalte aufwiesen, war die Veränderung vom ersten zum zweiten Standjahr bei der mehrjährigen Blühmischung sowohl in den TS-Gehalten als auch beim Teilindex TS-Gehalt zu erkennen. Die mehrjährigen Stauden schaffen es offenbar eher, ausreichend abzureifen und das sogar bei einem vergleichsweise frühen Erntetermin Mitte August, wie im vorliegenden Versuch beobachtet. Aus arbeitswirtschaftlicher Sicht ist dies nicht zuletzt positiv für die Entzerrung von Arbeitsspitzen zu bewerten. Zudem bleibt noch ausreichend Zeit für den Wiederaustrieb. Auch von Cossel \& Lewandowski (2016) fanden niedrige TS-Gehalte im ersten Standjahr bei zwei, aus unterschiedlichen Arten zusammengesetzten mehrjährigen Blühmischungen und genau wie im vorliegenden Versuch, höhere TS-Gehalte in späteren Standjahren. Die Autoren begründeten dies mit der nur geringen Anzahl an bestandsdominierenden Arten, die sich in den späteren Standjahren durchgesetzt haben und der damit einhergehenden einfacheren Ermittlung optimaler TS-Gehalte als bei einer diversen Mischung. Werden hingegen die Blühmischungen als Untersaat in Mais etabliert, werden die einjährigen Arten zusammen mit Mais geerntet. Hier wird der TS-Gehalt vom Mais als dominierende Art bestimmt und so u.a. wirksam dem Problem niedriger TS-Gehalte im ersten Standjahr begegnet (Vollrath 2012, von Cossel et al. 2019).

Einen ebenfalls deutlich negativen Wert beim Teilindex TS-Gehalt zeigte auch die Durchwachsene Silphie am Standort Reinshof aufgrund des geringen TS-Gehalts von nur etwa $22 \%$ bei der Ernte Ende August. Bei keiner anderen Variante war der Unterschied zwischen den Standorten hinsichtlich der TS-Gehalte so groß wie bei der Durchwachsenen Silphie. Denn am 
Standort Schoningen erreichte diese Variante mit einem optimalen TS-Gehalt von $35 \%$ einen deutlich positiven Indexwert. Dass dies höchstwahrscheinlich mit den bereits angesprochenen Trockenheitsschäden am Standort Schoningen und dem damit zusammenhängenden frühen Absterben der Pflanzen zusammenhängt, lassen auch die in der Literatur zu findenden TSGehalte bei der Durchwachsenen Silphie von meist z.T. deutlich unterhalb von $28 \%$ vermuten (Leonard Haag et al. 2015, Hartmann \& Lunenberg 2016). Auch Schoo et al. (2017) fanden TS-Gehalte von $30 \%$ nur bei starkem Trockenstress. Praxiserfahrungen deuten eher darauf hin, die Durchwachsene Silphie besser zeitig bei TS-Gehalten von $25 \%$ zu ernten, da bei späteren Ernteterminen und höheren TS-Gehalten auch der Ligninanteil zunimmt und damit die Methanausbeute sinkt (Karpenstein-Machan 2017). Ob dieser Zusammenhang auch bei der vorliegenden Untersuchung besteht, kann nicht abgeschätzt werden, da nur Ergebnisse für die spezifische Methanausbeute vom Standort Reinshof vorliegen (Tabelle 9). Mast et al. (2014) fanden bei einer Untersuchung verschiedener Erntetermine bereits in der zweiten Augustdekade TS-Gehalte von etwa $30 \%$. Erstaunlich ist dabei, dass dieser auch vier Wochen später nicht weiter anstieg. Das würde eher für eine hohe Flexibilität im Erntetermin sprechen. Auch der spezifische Methanertrag sank bei diesen Autoren mit späterem Erntetermin nicht, sondern stieg sogar noch etwas an. Dass die Konservierung der Durchwachsenen Silphie jedoch auch bei geringeren TS-Gehalten gelingen kann, schlussfolgerten Hartmann \& Lunenberg (2016): Bei TS-Gehalten zwischen 25 und $28 \%$ war die Biomasse gut silierbar und es traten kaum Sickersaftverluste auf.

Hohe positive Indexwerte hatte in beiden Versuchsjahren und an beiden Standorten der Mais und das Gemenge aus Amarant und Mais. Hier trat eher das Problem auf, dass die TS-Gehalte des Maises insbesondere im zweiten Versuchsjahr am Standort Schoningen sehr hoch waren. Mit über $40 \%$ kann möglicherweise mit Schwierigkeiten bei der Verdichtung des Silostapels gerechnet werden. Dennoch schlugen die hohen TS-Gehalte bei der Indexberechnung, wie bereits angesprochen, nicht negativ, sondern positiv zu Buche. Wie die Ergebnisse zeigen, ist jedoch die Erreichung ausreichend hoher TS-Gehalte schwieriger als die Vermeidung zu hoher TS-Gehalte, was letztlich durch den Erntetermin bestimmt wird und somit steuerbar ist. Es wird deutlich, dass derzeit hauptsächlich der Mais bestens an das Biogassystem und die dafür notwendige Silierung angepasst ist.

\subsubsection{Teilindex Methan}

Der in den Teilindex Methan eingehende Methanflächenertrag setzt sich zusammen aus dem Methanbildungsvermögen der Biomasse, also dem spezifischen Methanertrag, und dem Trockenmasseertrag je Flächeneinheit. Da es bei der Biogaserzeugung um den Energieertrag geht, ist der Methanflächenertrag eine wichtige Größe zur Bestimmung der Leistungsfähigkeit je Flächeneinheit einer Feldfrucht. Der Methanflächenertrag wird jedoch stark vom Trockenmasseertrag bestimmt. Mit einem Korrelationskoeffizienten von $r=0,981 \quad(p<0,001$, Tabelle 18) ist dieser Zusammenhang in der vorliegenden Untersuchung trotz der vielfältigen Varianten sehr eng. Gleiches stellten auch Roth (2010) bei Winterzwischenfrüchten (Winterackerbohnen, Wickroggen und Gemengen daraus) und von Cossel (2017) für Amarant und Mais fest. Je größer der Einfluss des Trockenmasseertrags auf den Methanflächenertrag, umso größer ist auch der Einfluss des Trockenmasseertrags auf den Teilindex Methan. So kommt es auch, dass sich die Werte des Teilindex Trockenmasseertrag und des Teilindex Methan sowohl in der Relation zueinander als auch in Bezug auf ihre absoluten Beträge bei den Varianten stark 
ähneln. Das bedeutet, dass der Trockenmasseertrag einmal über den Teilindex Trockenmasseertrag in den Index IrA eingeht und ein weiteres Mal durch sein starkes Gewicht am Teilindex Methan. Zu überlegen wäre daher, einen dieser beiden Teilindizes aus dem Index IrA auszuschließen.

Auch wenn der spezifische Methanertrag beim Methanflächenertrag kaum ins Gewicht fällt, unterscheiden sich die Varianten z.T. deutlich mit Werten zwischen 0,266 und 0,356 $\mathrm{Nm}^{3} \mathrm{CH}_{4}$ $\mathrm{kg}^{-1}$ oTM, wobei sich insbesondere der Mais mit den höchsten Werten auszeichnet. Es gibt jedoch Hinweise darauf, dass es bei Zumischung von Substraten mit geringem spezifischen Methanertrag zu Mais im Biogasfermenter zu Synergieeffekten kommt und der Methanertrag der Mischung höher ist als aus den spezifischen Methanerträgen der Einzelkomponenten zu erwarten wäre (Heidecker 2008, Sauer et al. 2018). So fand Heidecker (2008) einen um $11 \%$ höheren Methanertrag, wenn eine Mischung aus Mais (71\%), Gras (14\%), Triticale (10\%) und Stroh (5\%) vergoren wurde, im Vergleich zu den anteilig aufaddierten Methanerträgen bei Monovergärung der Einzelkomponenten. Demzufolge würden in der vorliegenden Untersuchung die Methanflächenerträge, die aus den spezifischen Methanerträgen unter Annahme der Monovergärung der Energiepflanzenvarianten berechnet wurden, bei Varianten mit geringen spezifischen Methanausbeuten die in der Fermentermischung tatsächlich erzielbaren Methanerträge unterschätzen. Das wäre ein Argument, den Teilindex Methanflächenertrag aus dem Index IrA herauszulassen und stattdessen die Leistungsfähigkeit der Varianten ausschließlich über den Teilindex Trockenmasseertrag abzubilden. Unterschiede in der spezifischen Methanausbeute zwischen den Varianten, die in Fermentermischungen möglicherweise nicht bestehen, würden dann bei der Bewertung auch nicht mehr ins Gewicht fallen.

Werden die einzelnen Feldfrüchte betrachtet, zeigt sich der geringste spezifische Methanertrag in der vorliegenden Untersuchung bei der Durchwachsenen Silphie mit 0,266 $\mathrm{Nm}^{3} \mathrm{CH}_{4}$ $\mathrm{kg}^{-1}$ oTM. Im Vergleich zu Mais, der Variante mit den höchsten spezifischen Methanerträgen, sind die Methanerträge der Durchwachsenen Silphie um $25 \%$ geringer. Biertümpfel \& Conrad (2013) konnten für die Durchwachsene Silphie einen Zusammenhang zwischen der Höhe der spezifischen Methanerträge und dem Erntetermin zeigen. Bei früher Ernte waren bei diesen Autoren bis zu 0,300 Nm${ }^{3} \mathrm{CH}_{4} \mathrm{~kg}^{-1}$ oTM zu finden, was den Abstand zu Mais deutlich verringern würde. Mit fortschreitender Entwicklung der Pflanzen nimmt der Anteil an Lignin zu und der Zuckergehalt ab, was sich negativ auf die spezifischen Methanerträge auswirkt (Biertümpfel \& Conrad 2013). Wenn aber, wie bereits diskutiert, die Methanhektarerträge weniger von den spezifischen Methanerträgen als vielmehr von den Trockenmasseerträgen beeinflusst werden, ist eine frühe Ernte bei hohen spezifischen Methanerträgen aber noch nicht voll ausgebildeten Trockenmasseerträgen eher nicht sinnvoll. So fanden Biertümpfel und Conrad (2013) die höchsten Methanhektarerträge nicht zur Ernte bei den höchsten spezifischen Methanerträgen der Durchwachsenen Silphie sondern zu einem späteren Erntetermin. Bis zu diesem nahm der spezifische Methanertrag durch eine zunehmende Lignifizierung zwar wieder ab, die Zunahme an Trockenmasseertrag konnte dies aber kompensieren bzw. übersteigen. Es gibt Überlegungen, die Silphie zweifach in einer Vegetationsperiode zu ernten, um die negativen Effekte der Lignifizierung zu umgehen. Der Gesamtertrag reicht dabei laut Gansberger et al. (2015) aber nicht an den Ertrag bei einmaliger Ernte zum Ende der Blüte heran. Zudem würden bei zweifacher Ernte in einem frühen Entwicklungsstadium die positiven Effekte der Blüte nicht wirksam werden. 
Vergleichsweise geringe spezifische Methanerträge unterhalb von $0,300 \mathrm{Nm}^{3} \mathrm{CH}_{4} \mathrm{~kg}^{-1}$ oTM zeigten in der vorliegenden Untersuchung neben der Durchwachsenen Silphie auch die Sommer- und Winterackerbohnen und die ein- und mehrjährige Blühmischung. Die geringen spezifischen Methanerträge der Ackerbohnen mit 0,284 $\mathrm{Nm}^{3} \mathrm{CH}_{4} \mathrm{~kg}^{-1}$ oTM bei Winter- und 0,299 $\mathrm{Nm}^{3} \mathrm{CH}_{4} \mathrm{~kg}^{-1}$ oTM bei Sommerackerbohnen können durch die Angaben der BiogasausbeutenDatenbank des LfL bestätigt werden (LfL 2020a). Auch Menke (2011) fand vergleichsweise geringe spezifische Methanerträge bei klein- und großkörnigen Leguminosen, darunter auch Winterackerbohnen. Allerdings wurden die Angaben bei diesem Autor auf die Trockenmasse bezogen und nicht die organische Trockenmasse und sind daher nicht direkt mit den eigenen Befunden vergleichbar. Kaiser \& Gronauer (2007) gehen davon aus, dass das Biogas-Ertragspotenzial proteinreicher Feldfrüchte unterschätzt wird. Laut Schnürer (2016) ist ein C/N-Verhältnis von 15 bis 25 in der Biomasse optimal für den Vergärungsprozess. Kleinere C/N-Verhältnisse führen zur Hemmung des Biogasprozesses bzw. der Mikroorganismen durch Ammoniak. Im hier vorliegenden Versuch hatten z.B. die Sommerackerbohnen ein $\mathrm{C} / \mathrm{N}-$ Verhältnis von 14 (Daten nicht dargestellt) und damit geringer als der von Schnürer (2016) angegebene Grenzwert für optimale Vergärung. Möglicherweise kam es im Biogasertragstest bei den Ackerbohnen des vorliegenden Versuchs zu einer Hemmung der Mikroorganismen durch Ammoniak und daraus resultierend zu geringen spezifischen Methanerträgen.

Neben Ammoniak sind proteinreiche Biogassubstrate häufige auch eine Quelle für die Bildung von Sulfiden, die nicht nur für die Mikroorganismen toxisch werden können, sondern auch Komplexe mit Metallen bilden, was zu einer verminderten Bioverfügbarkeit von Spurenelementen führen und dadurch der Biogasprozess wiederum ebenfalls gestört werden kann (Schnürer 2016). Dass Leguminosen aber offenbar tatsächlich ein höheres Methanbildungsvermögen haben als im vorliegenden Versuch gefunden wurde, zeigen Angaben der BiogasausbeutenDatenbank des LfL für z.B. ein Gemenge aus Erbsen und Wicken im Knospenstadium mit einem spezifischen Methanertrag von $0,346 \mathrm{Nm}^{3} \mathrm{CH}_{4} \mathrm{~kg}^{-1}$ oTM (LfL 2020a). Auch Roth (2010) konnte, möglicherweise durch methodischen Unterschiede bei der Analyse, einen deutlich höheren spezifischen Methanertrag bei Winterackerbohnen mit $0,344 \mathrm{Nm}^{3} \mathrm{CH}_{4} \mathrm{~kg}^{-1}$ oTM finden als in der vorliegenden Untersuchung. Damit sind diese Feldfrüchte gleichauf mit Mais. Kaiser \& Gronauer empfehlen, das C/N-Verhältnis bei der Eingabe der Substrate in den Fermenter zu korrigieren. Die bereits vorgeschlagene Beimischung von Getreidestroh zu Sommerackerbohnen könnte neben der Verbesserung der Silierfähigkeit von Sommerackerbohnen auch zur Erhöhung des C/N-Verhältnisses und damit zu einer Verbesserung bei der Vergärbarkeit beitragen.

\subsubsection{Teilindex Nmin}

Neben den Teilindizes, die die klassischen Schwerpunkte bei der Energiepflanzenproduktion ansprechen, also die Leistungsfähigkeit und Prozessparameter hinsichtlich der Substratkonservierung, sollen in dieser Arbeit aber auch ökologisch bedeutsame Wirkungen des Energiepflanzenanbaus bewertet werden. So soll der Teilindex Nmin Aufschluss darüber geben, inwieweit beim Anbau der untersuchten Varianten, Nmin im Boden zurückbleibt, das u.U. in gröBere Bodenschichten verlagert und ausgewachsen werden kann. Um das gesamte Anbausystem einer Variante abzubilden, gehen dabei sowohl die Nmin-Mengen im Boden im Winter als auch nach der Ernte in die Teilindexberechnung ein. Diese beiden Zeitpunkte wurden erfasst über den Teilindex Nmin (w) für den Nmin im Winter sowie den Teilindex Nmin (s/h) für den 
Nmin nach der Ernte (Werte für Teilindex Nmin (w) und Teilindex Nmin (s/h) im Anhang, Tabelle A4). Der Mittelwert dieser beiden Teilindizes bildet schließlich den Teilindex Nmin (Abschnitt 2.5.1). Da insbesondere die Winterniederschläge die Nitratauswaschung begünstigen (Fraser et al. 2013, Meisinger \& Ricigliano 2017), sind es insbesondere die Zeitpunkte über Winter sowie nach der Ernte im Herbst, in denen niedrige Nmin-Mengen im Boden vorhanden sein sollten. Dies gilt es im Teilindex Nmin zu bewerten. Bei den Varianten des Zweikulturnutzungssystems werden daher die Nmin-Mengen im Winter unter den Erstfrüchten sowie nach der Ernte des Maises als Zweitfrucht einbezogen. Bei den Sommerhauptfrüchten nach Schwarzbrache gehen entsprechend die Nmin-Mengen im Winter bei Schwarzbrache sowie nach der Ernte der Hauptfrüchte ein. Bei den mehrjährigen Varianten gehen wiederum die Werte im Winter, in diesem Fall das erste Jahr als Etablierungsjahr und das zweite Jahr als Dauerkultur, sowie nach der letzten Ernte vor dem Winter ein. Die Vergleichbarkeit der beiden Versuchsjahre ist bei diesen Varianten also nicht unbedingt gegeben.

Da in den Teilindex Nmin sowohl der Zustand im Winter als auch nach der Ernte eingeht, sind die Indexwerte nicht ohne die Nmin-Werte der einzelnen Termine zu verstehen. Auffällig sind zum einen die hohen negativen Indexwerte bei den Winterackerbohnen gefolgt von Mais an beiden Standorten und in beiden Versuchsjahren (Tabelle 19, Tabelle 20). Dies geht hervor aus den im Vergleich zu den anderen Erstfrüchten aber auch zur Schwarzbrache meist hohen Nmin-Mengen im Winter bei den Winterackerbohnen, insbesondere aber aus den hohen NminMengen nach der Ernte der Zweitfrucht Mais nach Winterackerbohnen (Abbildung 16, Abbildung 17, Abbildung 18, Abbildung 19). Das Problem hoher Nmin-Mengen im Winter beim Anbau von Winterackerbohnen stellte auch Menke (2011) fest. Ein negativer Wert für den Teilindex Nmin, wie inn auch dieser Autor beim Vergleich verschiedener Winterzwischenfrüchte fand, konnte bei den Winterackerbohnen in der vorliegenden Untersuchung also erwartet werden. Jedoch waren hier erst die Nmin-Mengen nach der Ernte des Maises als Zweitfrucht nach Winterackerbohnen ausschlaggebend für die recht hohen negativen Teilindexwerte.

Etwas anders, aber ebenfalls mehrheitlich in negativen Werten beim Teilindex Nmin mündend, verhielt es sich bei den übrigen Varianten des Zweikulturnutzungssystems. Diese waren überwiegend mal mehr mal weniger deutlich imstande, den Nmin im Boden bis zum Winter im Vergleich zur Schwarzbrache abzusenken, was den Varianten auch bei der Bewertung, in Form eines positiven Werts für den Teilindex Nmin (w), zugutekam. Die Stärke vieler Winterzwischenfrüchte, Stickstoff über Winter in der Biomasse zu konservieren und damit vor Auswaschung zu schützen, ist, in Abhängigkeit aber von der Pflanzenart, Saatzeit u.a., bekannt (Menke 2011, Fraser et al. 2013, Meisinger \& Ricigliano 2017, Thapa et al. 2018). Durch die Einbeziehung der Nmin-Mengen nach der Ernte der Zweitfrucht Mais jedoch, wurden die Vorteile der Erstfrüchte, bis zum Winter Nmin abzusenken, durch die vergleichsweise hohen Nmin-Mengen nach der Maisernte als Zweitfrucht - sichtbar am negativen Wert für den Teilin$\operatorname{dex} \operatorname{Nmin}(\mathrm{s} / \mathrm{h}$ ) - überdeckt (Tabelle A4). Dies resultierte in größtenteils negativen Werten für den Teilindex Nmin bei den Varianten des Zweikulturnutzungssystems und damit in einer negativen Bewertung des Anbausystems bei dieser Kenngröße.

Laut Stülpnagel et al. (2009) dienen die Nmin-Mengen nach der Ernte als Indikator für eine angepasste N-Düngung. Bis auf die ein- und mehrjährige Blühmischung am Standort Schoningen, bei denen auch aufgrund der bereits beschriebenen Schwierigkeiten in der Bestandsführung wenig Biomasse geerntet werden konnte, nahmen alle untersuchten Feldfrüchte mehr 
Stickstoff auf, als innen über die Düngung zur Verfügung stand (Daten nicht dargestellt) - so auch die Varianten mit negativen Werten beim Teilindex Nmin. Wie stark die Nmin-Mengen im Herbst nach Maisanbau in der Praxis schwanken können, zeigen Ergebnisse von Seitz \& Drewes (2019) für Niedersachsen. Bei der Untersuchung von 57 Praxisschlägen fanden diese Autoren Nmin-Mengen nach der Maisernte zwischen 32 und $219 \mathrm{~kg} \mathrm{~N}^{-1}$ bzw. durchschnitt-

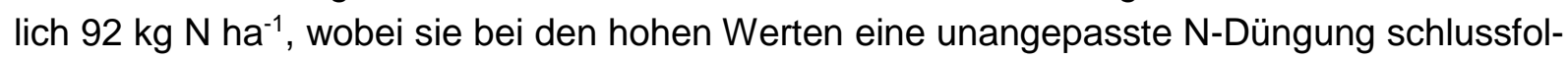
gerten. Wie ein langjähriges Nmin-Monitoring nach der Ernte von Mais in Bayern zeigt, konnten innerhalb von zehn Jahren die Nmin-Mengen nach Mais von über $120 \mathrm{~kg} \mathrm{~N}^{-1}$ Anfang der 1990er Jahre auf etwa $60 \mathrm{~kg} \mathrm{~N} \mathrm{ha}^{-1}$ im Jahr 2000 reduziert werden (LfL 2020b). Damit können, mit Ausnahme des Zweikulturnutzungssystems aus Winterackerbohnen und Mais, die in der vorliegenden Untersuchung nach der Ernte im Boden zurückgebliebenen Nmin-Mengen, insbesondere am Standort Reinshof von etwa $25-50 \mathrm{~kg} \mathrm{~N}^{-1}$ bei den Mais-Varianten (MaisReinsaat in Hauptfruchtstellung, Mais im Gemenge mit Amarant und Mais als Zweitkultur) als recht günstig eingestuft werden (Abbildung 16, Abbildung 17). Am Standort Schoningen traf dies auf Mais in Hauptfruchtstellung, das Gemenge aus Amarant und Mais sowie zumindest überwiegend auch auf den Mais als Zweitfrucht ebenfalls zu. Wie am Standort Reinshof, zeigte auch am Standort Schoningen der Mais nach Winterackerbohnen besonders hohe Nmin-Mengen nach der Ernte. Hier hinterließ jedoch auch der Mais nach Wintertriticale zumindest im zweiten Versuchsjahr mehr Nmin im Boden als am Standort Reinshof bei dieser Varainte.

Die Nmin-Mengen nach der Ernte von Mais nach Winterackerbohnen im Zweikulturnutzungs-

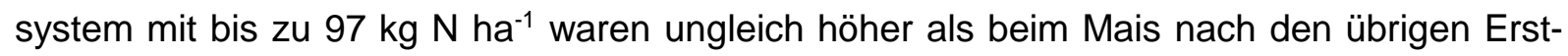
früchten. Der Mais als Zweitfruchtmais wurde nach Praxisempfehlung gedüngt, wobei eine geringere Ertragserwartung im Vergleich zu Mais in Hauptfruchtstellung eine Reduzierung der N-Düngemenge diesem gegenüber nach sich zog (Baumgärtel \& Eiler 2012). Zudem wurde die vorgesehene N-Düngemenge um die Nmin-Menge nach der Ernte der Winterackerbohnen in der jeweiligen Umwelt reduziert. Dennoch war hier die N-Düngung zu Mais nach Winterackerbohnen offenbar überzogen, denn die an den vergleichsweise hohen Nmin-Mengen ersichtliche höhere N-Verfügbarkeit dieser Variante brachte keine Ertragsvorteile in der oberirdischen Biomasse gegenüber dem Mais nach den übrigen Erstfruchtvarianten. KarpensteinMachan \& Stülpnagel (2000) konnten beim Zweikulturnutzungssystem aus Wintererbsen als Erst- und Mais als Zweitfrucht einen vergleichbaren Maisertrag finden, wenn der Mais entweder nicht gedüngt oder mit $75 \mathrm{~kg} \mathrm{~N} \mathrm{ha}^{-1}$ gedüngt wurde. Bei weiter gesteigerter Düngung auf maximal $225 \mathrm{~kg} \mathrm{~N} \mathrm{ha}^{-1}$ konnte sogar der Ertrag der ungedüngten Variante nicht erreicht werden. Allerdings war bei diesen Autoren der Trockenmasseertrag des Maises als Zweitfrucht mit etwa $90 \mathrm{dt} \mathrm{TM} \mathrm{ha}^{-1} \mathrm{z}$.T. deutlich geringer als in der vorliegenden Untersuchung mit etwa

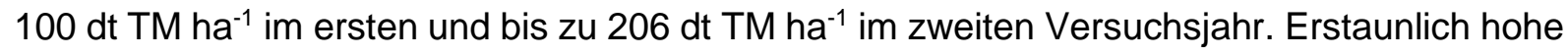
Mais-Zweitfrucht-Erträge erzielten Menke (2011) nach Winterackerbohnen ohne zusätzliche

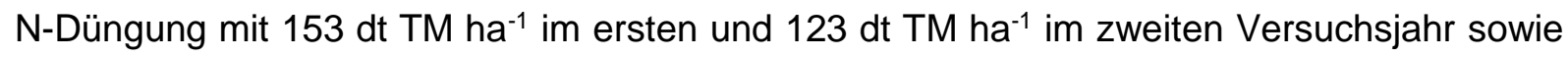
Roth (2010) mit, in Abhängigkeit vom Saattermin der Zweitfrucht, zwischen 110 und 185 dt TM $\mathrm{ha}^{-1}$. Sowohl diese, als auch die eigenen Befunde hinsichtlich der Nmin-Mengen nach Mais als Zweitfrucht nach Winterackerbohnen sprechen dafür, die N-Düngung zu Mais nach Winterackerbohnen weiter zu reduzieren als es in der vorliegenden Untersuchung der Fall war. Dies würde die Bewertung des Zweikulturnutzungssystems aus Winterackerbohnen und Mais sowohl im Teilindex Nmin durch die Reduzierung der Nmin-Mengen nach Mais als auch im Teilindex Ökonomische Aspekte durch die Einsparung von N-Düngemitteln verbessern. Wie Roth (2010) zeigen konnte, hinterließ der ungedüngte Mais als Zweitfrucht nach Winterackerbohnen 
nur geringe Nmin-Mengen im Boden. Anders als in der vorliegenden Untersuchung, in der der Mais als Zweitfrucht nach Winterackerbohnen gedüngt wurde, konnte der von Roth (2010) untersuchte Zweitfruchtmais also den residualen Stickstoff nach Winterackerbohnen gut nutzen, um vergleichsweise hohe Erträge zu erzielen.

Auf eine unzureichende Anrechnung der N-Mengen, die aus eingearbeiteten Zwischenfrüchten frei werden, und die damit zusammenhängende Gefahr der Verschiebung von N-Überschüssen in der Fruchtfolge, weisen auch Flessa et al. (2014) hin. Bedingt durch die späte Ernte des Maises, kann auch die Aussaat der Folgefrucht erst spät erfolgen. Die Aufnahme hoher Nmin-Mengen vor Winter ist daher unwahrscheinlich. Menke (2011) fand bei einem Saattermin Mitte Oktober N-Aufnahmen von Getreide bis zum Ende der Vegetationszeit von höchstens $4 \mathrm{~kg} \mathrm{~N} \mathrm{ha-1.}$

Meinen et al. (2019) untersuchten ein Anbausystem für Winterackerbohnen, das dem Problem hoher Nmin-Mengen über Winter bei Winterackerbohnen entgegenwirken soll: Den Winterackerbohnen wird dabei eine Hafer-Zwischenfrucht vorangestellt, in die die Winterackerbohnen über Direktsaat gesät werden. Dies, so konnten die Autoren zeigen, bewirkt eine Reduzierung der Nmin-Mengen vor Winter im Vergleich zur Variante ohne Hafer-Zwischenfrucht. Dies würde die Bewertung des Zweikulturnutzungssystems aus Winterackerbohnen und Mais im Teilindex Nmin (w) verbessern. Inwieweit sich die Mineralisation des Stickstoffs aus der über Winter abgestorbenen Hafer-Biomasse auf die Nmin-Mengen nach der Ernte der Zweitfrucht Mais auswirken würde, ist nicht bekannt. Bei Mineralisation in der Vegetationszeit des Maises könnte der Stickstoff, der ohne den Anbau der Hafer-Zwischenfrucht möglicherweise der Auswaschung unterlegen hätte, neben der Mineralisation aus den Ackerbohnenrückständen als weitere Stickstoffquelle für den Mais dienen, was wiederum weitere N-Düngemitteleinsparungen zu Mais nach sich ziehen könnte.

Ebenfalls überwiegend negative Werte beim Teilindex Nmin wiesen die Sommerackerbohnen auf. Einmal wurde angenommen, dass die Sommerackerbohnen nach Schwarzbrache über Winter angebaut werden. Der aus dem Winter Nmin bei Schwarzbrache konstruierte Teilindex Nmin (w) ist daher bei den Sommerackerbohnen, wie auch bei den übrigen Sommerhauptfrüchten nach Schwarzbrache, negativ (Tabelle A4). Auch nach der Ernte der Sommerackerbohnen blieb meist deutlich mehr Stickstoff im Boden zurück als bei anderen Varianten - und das bei einem vergleichsweise frühen Erntetermin im Juli. Bis zum Herbst kann hier wahrscheinlich mit noch wesentlich höheren N-Mengen durch Mineralisation der Ernte- und Wurzelrückstände gerechnet werden. Das verhältnismäßig enge $\mathrm{C} / \mathrm{N}-$ Verhältnis der Wurzeln (Tabelle 13, Tabelle 14) und die hohe Menge an in den Wurzeln gespeichertem Stickstoff (Abbildung 14, Abbildung 15) lassen dies erwarten. Würden bei den Sommerackerbohnen die NminMengen zum eigentlich für die Nitratauswaschungsgefahr relevanten Zeitpunkt im Herbst als Grundlage für die Indexberechnung verwendet, würden die Sommerackerbohnen wahrscheinlich im Teilindex Nmin noch deutlich schlechter gestellt. Eine Folgefrucht, die hohe Mengen Stickstoff vor Winter aufnehmen kann, könnte dies allerdings relativieren. Infrage kommt hier der Winterraps, dessen vergleichsweise frühe Saatzeit gut mit der Ganzpflanzenernte der Sommerackerbohnen übereinstimmen würde. Mit N-Aufnahmen bis zum Vegetationsende von 40 bis $60 \mathrm{~kg} \mathrm{~N} \mathrm{ha}^{-1}$ (Menke 2011) wäre auf der einen Seite Winterraps imstande, den Nmin im 
Boden nach Sommerackerbohnen abzusenken. Auf der anderen Seite könnte trotz der Restriktionen bei der N-Düngung zu Winterraps (Hogrefe 2020) eine gute Vorwinterentwicklung gefördert werden, um hohe Rapserträge zu sichern.

Hohe positive Werte beim Teilindex Nmin hatten insbesondere im zweiten Versuchsjahr die mehrjährigen Arten. Sowohl im Winter als auch nach der Ernte wiesen das Deutsche Weidelgras, die mehrjährige Blühmischung und die Durchwachsene Silphie nur geringe Nmin-Mengen von im zweiten Versuchsjahr meist weniger als $10 \mathrm{~kg} \mathrm{~N}^{-1}$ am Standort Reinshof und 20 $\mathrm{kg} \mathrm{N} \mathrm{ha}^{-1}$ am Standort Schoningen im Boden auf. Die Gefahr der Nitratauswaschung war bei diesen Varianten damit gering. So waren die mehrjährigen Arten auch die einzigen Varianten, bei denen im zweiten Versuchsjahr sowohl der Teilindex (w), der den Nmin über Winter bewertet, als auch der Teilindex (s/h), der den Nmin nach der Ernte bewertet, deutlich positiv (Tabelle A4). Im ersten Versuchsjahr, dem Etablierungsjahr, wurden diese Varianten nach Schwarzbrache angebaut, sodass sie hier im Winter im Teilindex (w) negativ bewertet wurden.

Der in der Vegetationszeit applizierte Stickstoff wurde bei den mehrjährigen Arten offenbar vollständig zur Bildung von Biomasse genutzt. Das legt allerdings auch die Frage nahe, ob eine höhere N-Düngung höhere Trockenmasseerträge hätte hervorbringen können. Neben der Düngung fanden die Pflanzen aber auch Stickstoff, der aus der organischen Substanz im Boden mineralisiert wurde, sichtbar an den höheren N-Aufnahmen als über die N-Düngung zur Verfügung stand, vor. Im Verhältnis zur gedüngten N-Menge viel $\mathrm{N}$ aufgenommen (Daten nicht dargestellt, Abbildung 10, Abbildung 11) und damit auch nur wenig Nmin im Boden hinterlassen, hat auch der Amarant. Anders als z.B. das Deutsche Weidelgras oder die Durchwachsene Silphie hat der Amarant den Stickstoff allerdings nicht für die Bildung hoher Trockenmasseerträge genutzt. Das spricht zwar für eine schlechte N-Verwertungseffizienz aber hinsichtlich einer Empfehlung für Wasserschutzgebiete wäre der Amarant demzufolge neben insbesondere den mehrjährigen Arten interessant. Würde der Amarant nicht nach Schwarzbrache angebaut, sondern, wie bereits an anderen Stelle diskutiert, im Zweikulturnutzungssystem nach einer über Winter Nmin absenkenden Erstfrucht, könnte die Bewertung des Amarants im Teilindex Nmin weiter gesteigert werden.

\subsubsection{Teilindex Wurzeltrockenmasse}

Der Teilindex Wurzeltrockenmasse soll eine Einschätzung über die Humusreproduktionsleistung von Energiepflanzen für die Biogaserzeugung ermöglichen. Die Humusreproduktion beim Anbau von Feldfrüchten erfolgt größtenteils über Ernte- und Wurzelrückstände, die auf dem Feld verbleiben (Kasper et al. 2015). Kätterer et al. (2011) konnten anhand eines Dauerfeldversuchs zeigen, dass die durch Wurzeln gebildete Humusfraktion im Boden beständiger ist als die aus der oberirdischen Biomasse stammende. Damit haben die Wurzeln eine große Bedeutung. Die Humusreproduktion aus Wurzeln betrifft ganz besonders den Energiepflanzenanbau zur Biogaserzeugung, da hier der nahezu gesamte oberirdische Aufwuchs der Energiepflanzen abgefahren wird.

Die Werte für den Teilindex Wurzeltrockenmasse hatten am Standort Schoningen in beiden und am Standort Reinshof im zweiten Versuchsjahr die mit Abstand größte Differenz zwischen den Varianten eines Parameters (Tabelle 19, Tabelle 20). Dabei gibt es sowohl große positive 
als auch große negative Indexwerte. Das bedeutet, dass sich die Varianten bei keinem anderen Parameter so stark unterschieden wie bei der Wurzeltrockenmasse. So hinterließen z.B. das Deutsche Weidelgras am Standort Reinshof und die Sommerackerbohnen am Standort Schoningen die 20-fache Menge Wurzeln nach der Ernte im Boden im Vergleich zum Amarant (Abbildung 12, Abbildung 13).

Aufgrund einer unterschiedlichen Tiefe bei der Wurzelprobenahme sind die Daten der beiden hier untersuchten Standorte nicht unmittelbar vergleichbar. Am Standort Schoningen machte der hoher Steingehalt unterhalb von $30 \mathrm{~cm}$ Tiefe die Probenahme unmöglich. Es ist anzunehmen, dass dies auch Auswirkungen auf das Wurzelwachstum an diesem Standort hat, was aber nicht quantifiziert werden kann.

Nur geringe Wurzeltrockenmassen zeigte auch der Mais als dominierende Energiepflanze zur Biogaserzeugung. Damit wird der Mais beim Teilindex Wurzeltrockenmasse deutlich negativ bewertet. Dass der Silomais als stark humuszehrende Feldfrucht eingestuft wird (VDLUFA 2014), braucht angesichts der geringen Rückführung organischer Substanz in den Boden nicht zu wundern. Allerdings werden bei dieser Kategorisierung weitere Faktoren einbezogen, z.B. die mit der Feldfrucht zusammenhängende Intensität der Bodenbearbeitung (VDLUFA 2014). In einer Untersuchung zum Effekt des Zuchtfortschritts bei Mais gingen Herrmann et al. (2018) u.a. der Frage nach, ob sich neben der oberirdischen Biomasse auch die unterirdische Biomasse von Mais im Laufe der Zeit verbessert hat und modernere Maissorten eine günstigere Humusbilanz aufweisen als ältere. Bei den von diesen Autoren untersuchten Sorten, mit Zulassung zwischen 1971 und 2012, konnte dies jedoch nicht bestätigt werden. Die Autoren fanden aber heraus, dass mit zunehmendem Zulassungsjahr die Wurzelseneszenz von der Blüte bis zur Siloreife abnahm, die jüngeren Sorten also weniger Wurzelmasse in dieser Zeit abbauten als die älteren. Diese längere Zeit mit intakten Wurzeln der jüngeren Sorten und die damit verbundene länger mögliche Nährstoffaufnahme kann zum Zuchtfortschritt bei der oberirdischen Biomasse mit zunehmendem Zulassungsjahr von Mais geführt haben. Dies ändert aber nichts an der insgesamt geringen Wurzelmasse des Maises. Interessanterweise waren die bei Herrmann et al. (2018) ermittelten absoluten Mengen an Wurzelrückständen zur Siloreife mit denen der vorliegenden Untersuchung vergleichbar.

Wie die vorliegende Untersuchung anhand der Wurzeltrockenmasseerträge und Werte für den entsprechenden Teilindex zeigt, verbessert sich die Bewertung des Maises aber deutlich, wenn er zusammen mit einer Winterung im Zweikulturnutzungssystem angebaut wird. Denn neben den Wurzelrückständen des Maises werden dann auch die der Erstfrüchte in die Indexberechnung einbezogen. Anders als beim oberirdischen Trockenmasseertrag, bei dem der Mais den Gesamtertrag und damit den Wert für den Teilindex Trockenmasseertrag des Zweikulturnutzungssystems wesentlich beeinflusst, sind es hier überwiegend die Erstfrüchte, die die Verbesserung bewirken. Diese Erhöhung in der Rückführung organischer Substanz durch das Zweikulturnutzungssystem kann zu einer deutlich besseren Einschätzung hinsichtlich der Humuswirksamkeit des Maisanbaus führen. Was aber die Ergebnisse des vorliegenden Versuchs auch zeigen, ist die nicht gleiche Eignung des Zweikulturnutzungssystems an allen Standorten z.B. aufgrund begrenzter Wärmesummen oder unzureichender Wasserversorgung. 
Auffällig ist auch die insbesondere im zweiten Versuchsjahr gefundene Wurzeltrockenmasse bei Mais in den Varianten des Zweikulturnutzungssystems. Im Vergleich zum ersten Versuchsjahr waren diese hier nach Wickroggen, Wintertriticale und dem Gemenge aus Winterackerbohnen und Wintertriticale höher (Abbildung 12). Möglicherweise hängt dies mit dem Wechsel der angebauten Maissorte zusammen. Fraglich wäre dann allerdings, warum der Mais nach Winterackerbohnen dieses Muster nicht zeigt. Möglicherweise könnten aber auch bei der Zweitfrucht Wurzelrückstände der Erstfrüchte miterfasst worden sein. Die Wurzeln der Winterackerbohnen waren durch das enge $\mathrm{C} / \mathrm{N}$-Verhältnis (Tabelle 13, Tabelle 14) nach der Ernte des Maises möglicherweise bereits stärker abgebaut als die Wurzeln der übrigen Erstfrüchte und waren daher nicht mehr in der Probe zu finden. Dies wiederum würde mit den deutlich höheren Nmin-Mengen im Boden nach Mais, der als Zweitfrucht auf Winterackerbohnen folgte im Vergleich mit den anderen Varianten dieses Systems, übereinstimmen. Das Problem dabei wäre, dass im Falle der mehrfachen Erfassung der Wurzelmasse bei der überwiegenden Zahl der untersuchten Varianten des Zweikulturnutzungssystems die Wurzeltrockenmasse überschätzt wäre, was schließlich auch zu ungerechtfertigt günstigen Werten beim Teilindex Wurzeltrockenmasse führen würde. Eine weitere Erklärung für diesen Befund könnte aber auch die Tatsache sein, dass der Mais nach Winterackerbohnen höhere N-Mengen aus der Mineralisation der Wurzelmasse vorfand und daher keine große Wurzelmasse benötigte, um den Boden nach Stickstoff zu erschließen.

Die erfasste Menge an Wurzeln bei den Erstfrüchten des Zweikulturnutzungssystems schwankte zwischen den Versuchsjahren, wobei insbesondere die getreidebetonten Varianten hier im zweiten Versuchsjahr schwächer waren als im ersten. Dies spiegelt auch die vom ersten zum zweiten Versuchsjahr rückläufigen Trockenmasseerträge der oberirdischen Biomasse bei gleicher N-Verfügbarkeit in beiden Jahren wider. Dass die Winterackerbohnen und am Standort Schoningen auch das Gemenge aus Winterackerbohnen und Wintertriticale vom ersten zum zweiten Versuchsjahr an Wurzeltrockenmasse zulegten, kann möglicherweise mit der hohen Unsicherheit, sichtbar an der großen Standardabweichung, erklärt werden (Abbildung 12, Abbildung 13). Auf Schwierigkeiten und Unsicherheiten bei der Wurzelprobenahme weist Anthes (2005) hin. Besonders bei Anbausystemen mit geringer Individuenzahl pro Fläche, wie es bei Ackerbohnen der Fall ist, sei diesem Autor zu Folge in Abhängigkeit des Beprobungsortes mit großen Abweichungen in der Wurzelmasse zu rechnen.

Die in der vorliegenden Untersuchung erfassten Wurzeltrockenmasseerträge der Erstfrüchte, insbesondere die der Winterackerbohnen mit bis zu $43 \mathrm{dt} \mathrm{TM} \mathrm{ha-1}$, sind vergleichsweise hoch, stimmen aber in ihrer Rangfolge zueinander in etwa mit denen von Menke (2011) überein, bei dem zumindest in einem Versuchsjahr die Winterackerbohnen deutlich mehr Wurzelmasse zeigten als der Wickroggen und die Wintertriticale. Streit et al. (2019) fanden Wurzeltrockenmassen bei Gemengen verschiedener Winterackerbohnen-Genotypen und Winterweizen von bis zu etwa $14 \mathrm{dt} \mathrm{TM} \mathrm{ha-1}$. Die Winterackerbohnen-Reinsaaten lagen bei diesen Autoren sogar darunter. Allerdings blieben hier, anders als in der vorliegenden Untersuchung, die Pfahlwurzeln der Ackerbohnen unberücksichtigt. Für ein hohes Potenzial von Ackerbohnen, organische Substanz in den Boden zu bringen, spricht auch der hohe Wurzeltrockenmasseertrag der Sommerackerbohnen. Die bereits in den oberen 0-30 cm mit bis zu $48 \mathrm{dt} \mathrm{TM} \mathrm{ha-1} \mathrm{eingetragene}$ Menge am Standort Schoningen weist darauf hin, dass der Anteil des oberen, kräftig ausgeprägten Teils der Pfahlwurzeln an der erfassten Wurzelmasse recht groß ist und dies die, im Vergleich mit Angaben aus der Literatur, gefundenen hohen Wurzelmassen ausmacht. 
Sehr viel Wurzelbiomasse mit Werten zwischen 42 und $63 \mathrm{dt} \mathrm{TM} \mathrm{ha}{ }^{-1}$ am Standort Reinshof und 30 dt TM ha-1 am Standort Schoningen hinterließ auch das Deutsches Weidelgras im Boden (Abbildung 12, Abbildung 13). Auch wenn das Deutsche Weidelgras hier als mehrjährige Feldfrucht untersucht wurde, wurde die Wurzelmasse zu einem theoretisch möglichen Umbruchszeitpunkt nach dem letzten der drei Schnitte im jeweiligen Jahr erfasst. Deutlich erkennbar ist eine Steigerung der Wurzeltrockenmasse vom ersten zum zweiten Versuchsjahr am Standort Reinshof um mehr als $20 \mathrm{dt} \mathrm{TM} \mathrm{ha-1}$. Am Standort Schoningen, an dem aufgrund einer späteren Aussaat des Weidelgrases nur eine einjährige Beprobung möglich war, war der Wurzeltrockenmasseertrag deutlich geringer. Hier wurden die Wurzeln aber nur bis zu einer Tiefe von $30 \mathrm{~cm}$ anstatt $60 \mathrm{~cm}$ am Standort Reinshof erfasst. Ein gewisser Unterschied ist daher zu erwarten. Vergleichbar mit den hohen Wurzeltrockenmassen des Deutschen Weidelgrases im vorliegenden Versuch waren auch die von Menke (2011) ermittelten Werte mit 46 bzw. $66 \mathrm{dt} \mathrm{TM} \mathrm{ha-1.} \mathrm{Allerdings} \mathrm{wurde} \mathrm{das} \mathrm{Deutsche} \mathrm{Weidelgras} \mathrm{bei} \mathrm{diesem} \mathrm{Autor} \mathrm{als} \mathrm{Winter-}$ zwischenfrucht angebaut und in beiden Versuchsjahren nach Herbstaussaat bereits im Frühjahr wieder umgebrochen bzw. die Wurzeltrockenmasse bestimmt. Auch Streit (2018) ermittelte bei einem einjährigen Bestand des Deutschen Weidelgrases eine Wurzeltrockenmasse von etwa 40 dt TM ha-1. Dabei war die Aussaatstärke des Weidelgrases im vorliegenden Versuch etwa vergleichbar mit der von Streit (2018). Bei Menke (2011) hingehen war die Aussaatstärke nahezu doppelt so hoch, was vermutlich mit der unterschiedlichen Nutzung als Winterzwischenfrucht im Gegensatz zur Nutzung als mehrjährige Kultur zusammenhängt.

Nur mäßig hohe Wurzeltrockenmasseerträge zeigte die Durchwachsene Silphie. Im Vergleich zu Mais brachte diese Variante zwar deutlich mehr Wurzelmasse in den Boden aber zumindest am Standort Reinshof wurde sie im Teilindex Wurzeltrockenmasse negativ bewertet. Die in der Literatur zu findenden hohen Wurzeltrockenmassen der Durchwachsenen Silphie konnten damit in der vorliegenden Untersuchung nicht bestätigt werden. So konnten Schoo et al. (2017) im ersten Jahr der Untersuchung 50 dt TM ha-1 und im zweiten Jahr 75 dt TM ha ${ }^{-1}$ ermitteln. Wie anhand dieser Befunde zu erkennen ist, kommt es offenbar mit zunehmender Standzeit als Dauerkultur zu einer nicht unbeachtlichen Zunahme an Wurzelmasse. Dazu kann aufgrund der einjährigen Ergebnisse des vorliegenden Versuchs keine Aussage getroffen werden. Auch Franzaring et al. (2015) weisen darauf hin, dass die Durchwachsene Silphie erst mit zunehmendem Alter einen ausgeprägten Wurzelstock entwickelt und auch erst dann ihre besondere Eigenschaft, trockenstresstolerant zu sein, entfaltet. Dass sie im ersten Standjahr nicht trockenstresstolerant ist, hat die vorliegende Untersuchung, wie bereits angesprochen, gezeigt. Versuche zu den Eigenschaften der Durchwachsenen Silphie über eine längere Standzeit stehen, wie die Literaturrecherche zeigt, bislang aus. Im Unterschied zu Schoo et al. (2017) wurde die Wurzeltrockenmasse in der hier vorliegenden Untersuchung zudem bei der Ernte zur Siloreife bestimmt und nicht im Stadium beginnender Blüte, in dem Schoo et al. (2017) zufolge das höchste Wurzelwachstum erreicht ist bevor es danach wieder rückläufig ist. Zudem unterschied sich die Methode bei der Trennung von Wurzeln und Boden. Während Schoo et al. (2017) die Wurzeln mit einem Sieb mit einer Maschenweite von 0,25 mm auffingen, wurde im vorliegenden Versuch ein Sieb mit $1 \mathrm{~mm}$ Maschenweite verwendet. Möglicherweise wurde dadurch ein Teil der Wurzelmasse hier nicht erfasst.

Angesichts hoher möglicher Wurzelrückstände bei der Durchwachsenen Silphie ist es überraschend, dass sie offenbar dennoch in ihrer Humusbilanz negativ eingeschätzt wird. Der von 
Brock et al. (2013) modellierte Humusbilanzkoeffizient liegt bei der Durchwachsenen Silphie bei $-588 \mathrm{~kg}$ Humus $\mathrm{C} \mathrm{ha}^{-1} \mathrm{a}^{-1}$ und ist damit nicht günstiger als der von Silomais nach Angaben der VDLUFA (2014) mit Werten zwischen -560 und -1040 Humus $C$ ha $^{-1} \mathrm{a}^{-1}$. Dabei nahmen Brock et al. (2013) mit 2,98 sogar ein deutlich engeres Spross/Wurzel-Verhältnis an als in der vorliegenden Untersuchung ermittelt wurde, gingen also von wesentlich höheren Wurzelmengen im Verhältnis zur oberirdischen Biomasse aus, als es im vorliegenden Versuch der Fall war. Wird aus den Spross- und Wurzeltrockenmasseerträgen (Abbildung 4, Abbildung 7, Abbildung 12, Abbildung 13) der vorliegenden Untersuchung ein Spross/Wurzel-Verhältnis für die Durchwachsene Silphie berechnet, so ergeben sich zum Erntetermin Werte von 9,61 am Standort Reinshof und 7,45 am Standort Schoningen. Erst unter Annahme einer Wurzeltrockenmasse von 75 dt TM ha-1 der Durchwachsenen Silphie, wie sie Schoo et al. (2017) ermittelten, wäre bei gleichbleibendem oberirdischen Trockenmasseertrag des Standorts Reinshof (221 dt TM ha-1), ein Spross/Wurzel-Verhältnis von 2,9, wie es Brock et al. (2013) annehmen, erreicht. Die von der VDLUFA (2014) vorgenommene Bewertung der Humuswirksamkeit beruht auf langjährigen Feldversuchen. Die Feststellung, dass für neue Anbausysteme, zu denen die Energiepflanzen gehören, Forschungsbedarf besteht, kann gut nachvollzogen werden.

Eine Steigerung der Wurzelbiomasse vom ersten zum zweiten Versuchsjahr wurde, ähnlich dem Deutschen Weidelgras und wie es bei der Durchwachsenen Silphie nach Befunden aus der Literatur angenommen werden kann, auch bei der mehrjährigen Blühmischung erwartet. Da die mehrjährigen Stauden in jedem Jahr aus den Reservestoffen der Wurzeln wieder neu austreiben müssen, wäre ein großes Wurzelsystem wahrscheinlich von Vorteil. Am Standort Schoningen gab es zwar einen Jahreseffekt mit höheren Wurzeltrockenmassen im zweiten als im ersten Versuchsjahr, aber insgesamt auf einem vergleichsweise niedrigen Niveau. Der erhebliche Unterschied zwischen den Versuchsjahren und die hohe Standardabweichung bei der einjährigen Blühmischung am Standort Reinshof zeigt, dass offenbar nicht nur die oben angesprochene geringe Individuenzahl je Flächeneinheit große Abweichungen bei den Wurzeldaten hervorbringen kann, sondern auch ein artenreicher Bestand.

Die an beiden Standorten geringste Wurzeltrockenmasse hinterließ der Amarant. Er bildete hier das Schlusslicht in der Bewertung mit einem deutlich negativen Wert für den Teilindex Wurzeltrockenmasse. In einer Untersuchung von Gimplinger \& Kaul (2008) lassen sich Angaben dazu finden, dass Amarant nur halb so viel Wurzeltrockenmasse bildet als Mais. Auch de Leon-Gonzalez et al. (2006) ermittelten Wurzeltrockenmassen mit im Mittel der Versuchsjahre bei Mais von 9,3 und bei Amarant von 4,6 dt TM ha ${ }^{-1}$ und damit genau halb so viel. In der vorliegenden Untersuchung war es z.T. sogar noch weniger als die Hälfte. Umso erstaunlicher sind die Stickstoffaufnahmen beim Amarant von bis zu $225 \mathrm{~kg} \mathrm{~N} \mathrm{ha}^{-1}$, die die vorgesehene

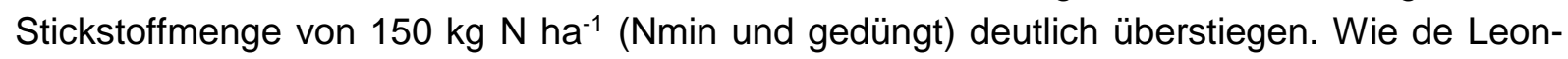
Gonzalez et al. (2006) aber feststellten, haben beim Amarant die Seitenwurzeln einen kleinen (1,3 $\mathrm{mm} \pm 0,4 \mathrm{~mm}$ bei Amarant und 2,9 $\mathrm{mm} \pm 1,0 \mathrm{~mm}$ bei Mais) und die Feinwurzeln einen sehr kleinen $(0,1 \mathrm{~mm} \pm 0,1 \mathrm{~mm}$ bei Amarant und 0,3 $\mathrm{mm} \pm 0,0 \mathrm{~mm}$ bei Mais) Durchmesser. Das spricht auf der einen Seite dafür, dass der Amarant auch mit geringer Wurzelmasse eine hohe Durchwurzelungsintensität hat und den Boden nach Nährstoffen erschließen kann. Auf der anderen Seite könnte es aber auch hier, wie bereits angesprochen, bei der in der vorliegenden Untersuchung angewandten Technik zu Verlusten an Wurzelmasse gekommen sein. 
Um abzuschätzen, ob die Wurzelmasse zum Aufbau von Dauerhumus beitragen kann, also mit einer längerfristigen Humuswirkung gerechnet werden kann oder ob es sich mehr um Nährhumus handelt, der rasch mineralisiert wird, ist die Qualität der Wurzelmasse ein wichtiges Kriterium. So wird Bauer \& Guggenberger (2020) zufolge organische Substanz im Boden mit einem $\mathrm{C} / \mathrm{N}-$ Verhältnis von kleiner 40 schnell zersetzt. Es wird dadurch nur wenig Humus aufgebaut. Aufgrund der, durch die einfach umsetzbare Substanz, gesteigerten mikrobiellen Aktivität, kann zusätzlich zum Abbau der frischen organischen Substanz auch Humus abgebaut werden. Wie die Ergebnisse des vorliegenden Versuchs zeigen, wiesen insbesondere die Wurzeln der Varianten, bei denen Leguminosen in Reinsaat oder Gemenge angebaut wurden aber auch die Durchwachsene Silphie, C/N-Verhältnisse von z.T. deutlich unterhalb von 40 auf (Tabelle 13, Tabelle 14). Dass hier eine schnelle Mineralisation der Wurzelmasse erfolgte, wird, wie bereits angesprochen, an den hohen Nmin-Mengen nach der Ernte des Maises, der als Zweitfrucht nach Winterackerbohnen angebaut wurde, ersichtlich (Abbildung 16, Abbildung 17, Abbildung 18, Abbildung 19). Dafür, dass diese hohen Nmin-Mengen aus der Mineralisation der Winterackerbohnenwurzeln stammen, spricht, dass der Mais als Zweitfrucht nach erstfruchtübergreifender Aufdüngung auf einen Sollwert bei allen Varianten in beiden Versuchsjahren innerhalb der beiden Standorte die gleiche Menge Stickstoff in der oberirdischen Biomasse akkumuliert hat (Abbildung 10, Abbildung 11). Eine höhere Anrechnung der Mineralisation und dementsprechend reduzierte N-Düngung des Maises als Zweitfrucht nach Winterackerbohnen wäre, wie bereits diskutiert, angebracht gewesen. Bei den Sommerackerbohnen ist aufgrund des engen $\mathrm{C} / \mathrm{N}$-Verhältnisses der Wurzelmasse und der hohen, in den Wurzeln gespeicherten N-Mengen (Abbildung 14, Abbildung 15) ebenfalls mit einer hohen N-Freisetzung nach der Ernte zu rechnen. Eine nach den Sommerackerbohnen angebaute Zwischenfrucht könnte diesen wahrscheinlich gut nutzen. Ein weiteres $\mathrm{C} / \mathrm{N}-\mathrm{Verhältnis} \mathrm{der} \mathrm{organischen}$ Substanz hingegen kann zu einer Anreicherung an Humus führen, da im Verhältnis zum Kohlenstoff weniger Stickstoff vorhanden ist, was die Vermehrung der Mikroben verlangsamt und damit den Abbau der kohlenstoffhaltigen Verbindungen verzögert.

Ein weiterer wichtiger Aspekt hinsichtlich des Humusaufbaus ist das Einbringen der organischen Substanz in tiefere Bodenschichten. Hier kann laut Bauer \& Guggenberger (2020) der Kohlenstoff längerfristig gespeichert werden, da mikrobielle Prozesse verlangsamt sind und der Abbau über Mineralisation gebremst ist. Eine längerfristige Humusspeicherung müsste demzufolge durch solche Feldfüchte begünstigt werden, die in der Lage sind, hohe Wurzelmassen auch in tiefen Schichten zu bilden bzw. zu hinterlassen. Die Varianten mit besonders hohen Wurzeltrockenmassen der vorliegenden Untersuchung, Winter- und Sommerackerbohnen, konzentrierten nahezu die gesamte Wurzelmasse in den oberen $30 \mathrm{~cm}$ im Boden (Daten nicht dargestellt). Nur die Durchwachsene Silphie mit etwa $5 \mathrm{dt}$ TM ha $^{-1}$ und das Deutsche Weidelgras mit etwa $4 \mathrm{dt} \mathrm{TM}^{-1}$ hinterließen auch nennenswerte Wurzelmengen in der Bodenschicht von 30-60 cm (Daten nicht dargestellt). Damit hinterließen diese Varianten mehr Wurzelmasse in tieferen Bodenschichten als der Amarant und z.T. der Mais im gesamten untersuchten Bodenraum von 0-60 cm. Schoo et al. (2017) fanden bei der Durchwachsenen Silphie $30 \%$ der Wurzeln unterhalb von $30 \mathrm{~cm}$ im Boden vor. Bei $75 \mathrm{dt} \mathrm{ha}^{-1}$ Wurzeltrockenmasse, wie die Autoren ermittelten, wären das $22,5 \mathrm{dt} \mathrm{TM} \mathrm{ha}^{-1}$ und damit eine beachtliche Wurzelmenge im Unterboden. Das auch von Ackergräsern ausgehende Potenzial, größere Mengen Wurzeln in tieferen Bodenschichten zu hinterlassen, bestätigte auch Menke (2011). Für die organische Substanz in der oberen Bodenschicht ist laut Bauer \& Guggenberger (2020) ein tiefes Einarbeiten in den Boden wichtig. Das betrifft insbesondere Biomasse mit einem 
engen $\mathrm{C} / \mathrm{N}$-Verhältnis, was in der vorliegenden Untersuchung insbesondere für die Wurzeln der Ackerbohnen zutrifft. Inwieweit die Ackerbohnenwurzeln dadurch vor Mineralisation geschützt werden können, bleibt zu untersuchen.

Gerade die beiden zuletzt angesprochenen Kenngrößen, C/N-Verhältnis und Durchwurzelungstiefe, müssten in einem Teilindex, der die Humuswirksamkeit abbilden soll, einbezogen werden. Der Teilindex Wurzeltrockenmasse könnte dahingehend erweitert werden. So könnte z.B. Wurzelmasse mit einem engen $\mathrm{C} / \mathrm{N}-$ Verhältnis mit einem Abschlag und solche mit einem weiten $\mathrm{C} / \mathrm{N}-$ Verhältnis mit einem Aufschlag in die Indexberechnung eingehen. Die Vorzüglichkeit des Deutschen Weidelgrases würde sich auch in einem erweiterten Teilindex zur Humuswirksamkeit zeigen, da sowohl die Wurzelmenge als auch das $\mathrm{C} / \mathrm{N}-$ Verhältnis und die Bodentiefe der Wurzelrückstände für einen guten Humusaufbau sprechen. Berücksichtigt werden könnte in einem erweiterten Teilindex auch die Gärrestrückführung sowie ein Bonus für die mehrjährigen Arten, bei denen keine Bodenbearbeitung stattfindet, was den Abbau von Humus vermindert bzw. den Humusaufbau begünstigt.

\subsubsection{Teilindex Bodenwasser}

Für die landwirtschaftliche Praxis ist es bedeutsam, Kenntnis über den Wasserzustand des Bodens zu haben, den eine Feldfrucht nach der Ernte zurücklässt. Dies hat nicht zuletzt Auswirkungen auf die Keimung und Entwicklung der Folgefrucht. Mithilfe des Teilindex Bodenwasser sollen daher die Varianten hinsichtlich der Beanspruchung des Bodenwasserhaushalts bewertet werden.

Neben der besseren Nutzung der Winterfeuchtigkeit zur Bildung von Biomasse durch das Zweikulturnutzungssystem besteht die Gefahr, dass bei ungünstiger Niederschlagsverteilung der Zweitfrucht nicht ausreichend Wasser zur Verfügung steht (Graß et al. 2013). Renius et al. (1992) zufolge verbrauchen Winterzwischenfrüchte bis Ende März keine größeren Wassermengen als die Brache. Danach jedoch steigt der Wasserverbrauch der Winterzwischenfrüchte stark an. Im hier vorliegenden Versuch wurde der Bodenwasserhaushalt im ersten Versuchsjahr durch die Erstfrüchte bis zur Ernte Anfang Juni deutlich beansprucht (Abbildung 20, Abbildung 21). Am Reinshof wurde der Boden in der Tiefe von 0-60 cm stark entleert, am Standort Schoningen sogar auf der gesamten untersuchten Tiefe von $0-80 \mathrm{~cm}$. Bei der Schwarzbrache dagegen waren zum gleichen Zeitpunkt deutlich höhere Wassermengen zu finden. Insbesondere am Standort Schoningen waren allerdings auch diese wesentlich geringer als - abgeleitet anhand der nutzbaren Feldkapazität - theoretisch möglich. Am Standort Reinshof war die Differenz zwischen der Schwarzbrache und der nutzbaren Feldkapazität nicht so groß wie am Standort Schoningen. Bei ausbleibenden Niederschlägen zur Aussaat der Zweitfrucht, wie es im ersten Versuchsjahr beobachtet wurde (Abbildung 2), ist unter diesen Bedingungen ein zügiger Feldaufgang und eine rasche Jugendentwicklung der Zweitfrucht gefährdet.

Bei einem Bodenwassergehalt von $<30 \%$ der nutzbaren Feldkapazität tritt Trockenstress für die Pflanzen ein (DWD 2016). Die im vorliegenden Versuch gemessenen nutzbaren Feldkapazitäten lagen im ersten Versuchsjahr nach den Erstfrüchten mit $15 \% \mathrm{nFK}$ in den oberen 30 $\mathrm{cm}$ am Standort Reinshof deutlich darunter. Am Standort Schoningen konnte in dieser Tiefe 
sogar überhaupt kein pflanzenverfügbares Wasser ermittelt werden. Hosseini et al. (2009) fanden in einem Topfversuch heraus, dass der Feldaufgang von Kichererbsen (Cicer arietinum L.) bei $25 \%$ Feldkapazität nur etwa $50 \%$ betrug und für das Auflaufen die doppelte Zeit im Vergleich zu besser mit Wasser versorgten Varianten benötigt wurde. Im Auflaufverhalten zwischen den bei 50 und 75 \% Feldkapazität ausgesäten Varianten konnten die Autoren hingegen keine Unterschiede finden. Beide lagen bei etwa $85 \%$ Feldaufgang. Der Mais als Hauptfrucht nach Schwarzbrache in der hier vorliegenden Untersuchung fand $58 \% \mathrm{nFK}$ am Standort Reinshof und $43 \% \mathrm{nFK}$ am Standort Schoningen vor und damit deutlich bessere Bedingungen hinsichtlich der Wasserversorgung als die Zweitfrucht. Die im Vergleich zum zweiten Versuchsjahr aber auch im Vergleich zu Mais als Hauptfrucht geringeren Trockenmasseerträge des Maises als Zweitfrucht im ersten Versuchsjahr liegen möglichweise auch in der mangelhaften Wasserversorgung zur Aussaat begründet.

Im zweiten Versuchsjahr hingegen unterschied sich zumindest am Standort Reinshof der Bodenwassergehalt nach den Erstfrüchten in der oberen Bodenschicht nur unwesentlich von der Schwarzbrache. Am Standort Schoningen war dies nicht ganz so deutlich, aber im Oberboden war auch hier nach den Erstfrüchten mehr Wasser vorhanden als im ersten Versuchsjahr. Anfang Juni des zweiten Versuchsjahres, einige Tage vor der Ernte der Erstfrüchte also, konnten Niederschlagsmengen von etwa $40 \mathrm{~mm}$ am Standort Reinshof und $30 \mathrm{~mm}$ am Standort Schoningen verzeichnet werden. Dies reichte offensichtlich aus, um den Bodenwasservorrat wieder aufzufüllen. Ohne diese Niederschläge wären angesichts des deutlichen Niederschlagsdefizits in den Frühjahrsmonaten nur geringe Bodenwassergehalte zu erwarten gewesen (Abbildung 2).

Ein Effekt der Erstfruchtvariante auf das nach der Ernte hinterlassene Bodenwasser lässt sich nicht eindeutig ausmachen. Am Standort Schoningen hinterließ die Wintertriticale tendenziell weniger Wasser im Boden als die übrigen Erstfrüchte, was hier möglicherweise erklärbar ist über die höheren Trockenmasseerträge dieser Variante im Vergleich mit den übrigen Erstfrüchten (Abbildung 4). Am Standort Reinshof zeigten sich im ersten Versuchsjahr etwas geringere pflanzenverfügbare Wassermengen nach Winterackerbohnen, was offenbar auch bis zur Zweitfruchternte (Mais nach Winterackerbohnen) nicht wieder aufgefüllt werden konnte. Im zweiten Versuchsjahr waren hier jedoch keine Unterschiede zwischen den Erstfrüchten zu erkennen.

Da es sich bei den Bodenwassergehalten zur Ernte der Varianten bzw. den daraus berechneten Mengen an pflanzenverfügbarem Wasser um Momentaufnahmen handelt, können nur Varianten mit gleichen Erntezeitpunkten miteinander verglichen werden. Im vorliegenden Versuch wurden die Varianten jedoch über einen langen Zeitraum geerntet, beginnend mit der Ernte der Erstfrüchte im Juni, über verschiedene Ernten im Laufe der Sommermonate z.B. die mehrjährige Blühmischung im August bis hin zur Ernte der Zweitfrucht und des Deutschen Weidelgrases (3. Schnitt) im Herbst. Neben den Varianten des Zweikulturnutzungssystems, bei denen sowohl die Erst- als auch Zweitfrüchte gleichzeitig geerntet wurden, war dies auch beim Gemenge aus Amarant und Mais und der Mais-Reinsaat sowie der Amarant-Reinsaat und der einjährigen Blühmischung der Fall. Wird das nach der Ernte im Boden zurückgebliebene Wasser von Amarant und der einjährigen Blühmischung verglichen, zeigt sich interessanterweise an beiden Standorten und in beiden Versuchsjahren, dass Amarant höhere Was- 
sermengen zurücklässt als die einjährige Blühmischung. Die Trockenmasseerträge waren dabei am Standort Reinshof bei diesen beiden Varianten etwa gleich und am Standort Schoningen war der Amarant sogar ertragreicher als die einjährige Blühmischung. Das spricht für den bereits angesprochenen geringen Wasserbedarf von Amarant (Kauffman \& Weber 1990). Die Höhe des zurückgebliebenen Wassers innerhalb der verschiedenen Versuchsjahre kann allerdings nicht ohne die Niederschlagsmengen in der Vegetationsperiode betrachtet werden. Im Falle des Amarants und der einjährigen Blühmischung fielen im zweiten Versuchsjahr im Laufe der Vegetationszeit etwa $60 \mathrm{~mm}$ weniger Niederschlag als im ersten Versuchsjahr, was auch die Unterschiede in der Wassermenge im Boden zwischen den Versuchsjahren erklärt.

Diese offenbar starke Abhängigkeit des Bodenwassers nach der Ernte von den Niederschlagsmengen sowie die unterschiedlichen Erntezeitpunkte machen eine Bewertung der Varianten auch anhand des Teilindex Bodenwassergehalt schwierig. So führen die Jahresunterschiede zu gegenläufigen Teilindexwerten. Im ersten Versuchsjahr waren die pflanzenverfügbaren Mengen an Wasser in den Sommerernten höher als zur Erstfruchternte im Frühjahr, was zu negativen Werten beim Teilindex Bodenwassergehalte der Varianten des Zweikulturnutzungssystems führte. Im zweiten Versuchsjahr hingegen waren die Bodenwassergehalte im Frühjahr höher als im Sommer, was wiederum zu positiven Teilindexwerten bei diesen Varianten führte (Tabelle 19, Tabelle 20). Bei keinem anderen Teilindex waren die Jahresunterschiede so stark ausgeprägt wie beim Teilindex Bodenwasser. Auch der Versuch, die Befunde zu verallgemeinern, indem der Index IrA mit den Teilindizes der beiden Versuchsjahre zusammengefasst wird, führt aufgrund der gegenläufigen Werte der einzelnen Jahre beim Teilindex Bodenwasser zur Aufhebung der Indexwerte (Abbildung 23, Abbildung 24). So kommt es auch, dass der Teilindex Bodenwasser am Standort Reinshof am Gesamtindex IrA im Mittel über alle Varianten nur einen Anteil von $3 \%$ ausmacht. Am Standort Schoningen ist es mit $6 \%$ etwas mehr. Aber hier gehen auch der Wickroggen und das Deutsche Weidelgras aufgrund der nur einjährig verfügbaren Daten ohne gemittelten Teilindexwert in IrA ein. Die Aussagekraft des Teilindex Bodenwasser stößt also an ihre Grenzen.

Am Standort Schoningen scheinen die Pflanzen zudem wesentlich stärker auf die Wasserversorgung über Niederschläge angewiesen zu sein als am Standort Reinshof. Die Bodenvorräte waren am Standort Schoningen insbesondere unterhalb von $30 \mathrm{~cm}$ Bodentiefe in beiden Versuchsjahren nur gering bzw. schnell erschöpft. Angesichts der in den Wintermonaten im langjährigen Mittel und teilweise auch im Versuchszeitraum beobachteten deutlich höheren Niederschläge am Standort Schoningen im Vergleich zum Standort Reinshof (Abbildung 2), liegt es nahe, am Standort Schoningen Varianten zu bevorzugen, die die Winterfeuchtigkeit effizient ausnutzen können. Vorteilhaft könnte dabei das in diesem Zusammenhang bereits diskutierte Deutsche Weidelgras sein. Zur Bewertung der Standorteignung einer Feldfrucht hinsichtlich der Wasserversorgung wäre hier eine Bilanz denkbar, die sich ergibt aus der Menge an Wasser, die nötig ist, um das Ertragspotenzial am Standort auszuschöpfen, und der standortspezifischen Niederschlagsmenge.

\subsubsection{Teilindex Spurenelemente}

Ziel des dieser Arbeit zugrundeliegenden Forschungsverbundprojekts war es, die Hypothese zu überprüfen, ob die Zugabe künstlich hergestellter Spurenelementpräparate zum Biogaspro- 
zess durch Zumischung spurenelementreicher Pflanzen ersetzt - oder teilweise ersetzt - werden könnte. Zur Bewertung des Beitrags einer Feldfrucht zur Spurenelementversorgung des Biogasfermenters - hier insbesondere zur Versorgung mit Cobalt- und Nickel - werden daher die untersuchten Varianten auch anhand ihrer Akkumulation der entsprechenden Elemente im Teilindex Spurenelemente abgebildet.

Dass Unterschiede in den akkumulierten Spurenelementmengen zwischen Pflanzenarten bestehen, ist bekannt. Zum Beispiel beschreibt Kabata-Pendias (2011) Pflanzenarten, die aufgrund ihrer sehr hohen Cobalt- und Nickelgehalte als Hyperakkumulatoren bezeichnet werden können. Solche Arten, z.B. Haumaniastrum sp. bei Cobalt oder Alyssum sp. bei Nickel, zeigen trotz tausendfacher und noch wesentlich höherer Spurenelementgehalte als die in der vorliegenden Arbeit gefundenen, keine Anzeichen toxischer Reaktionen. Auch Sauer et al. (2014) konnten Arten und darunter vor allem Arten zur landwirtschaftlichen Nutzung identifizieren, die zwar wesentlich geringere als Hyperakkumulatoren aber im Vergleich mit klassischen Energiepflanzen höhere Cobalt- und Nickelgehalte in ihrer oberirdischen Biomasse aufwiesen. Dazu zählten insbesondere Sommer- und Winterackerbohnen, Amarant, Malven, Buchweizen und Zottelwicken.

Eine artabhängige Cobalt- und Nickelakkumulation in der Biomasse konnte auch in der vorliegenden Untersuchung gefunden werden. Die Unterschiede zwischen den Varianten waren hier so groß, dass die Werte für den Teilindex Spurenelemente im Vergleich zu anderen Teilindexwerten mitunter zu den größten Differenzen zwischen größten und kleinsten Indexwerten führten, die Unterschiede in den Spurenelementaufnahmen also zwischen den untersuchten Varianten größer als die Unterschiede bei anderen erfassten Parametern waren. Die höchsten Gehalte konnten sowohl für Cobalt als auch für Nickel an den beiden Standorten Reinshof und Schoningen und in beiden Versuchsjahren insbesondere bei Sommer- und Winterackerbohnen gefunden werden (Tabelle 11). Diese Varianten lagen überwiegend ein Vielfaches über den klassischen Energiepflanzen Mais und Getreide und wie anhand der hohen positiven Teilindexwerte erkennbar auch deutlich über den mittleren Gehalten aller Varianten (Tabelle 19, Tabelle 20). Aber auch die ein- und mehrjährige Blühmischung sowie der Amarant konnten sich zumindest am Standort Schoningen mit im Vergleich zu Mais und den übrigen gräserbetonten Varianten hohen Cobaltgehalten auszeichnen. In hohen positiven Indexwerten resultierte dies jedoch nicht. Die Gründe hierfür liegen zum einen in der Berechnung der Teilindexwerte unter Verwendung der tatsächlichen Abfuhr von Cobalt und Nickel und der damit zusammenhängenden geringen Mengen aufgrund geringer Trockenmasseerträge dieser Varianten. Zum anderen ist der Teilindex Spurenelemente zusammengesetzt aus der Cobalt- wie auch Nickelabfuhr. Da die Nickelakkumulation in der Biomasse sich zwischen den Varianten nicht so stark unterschied wie die Cobaltakkumulation, hob der durch die Nickelabfuhr berechnete Beitrag zum Teilindex Spurenelemente den positiven Beitrag durch die Cobaltabfuhr bei einigen Varianten z.T. wieder auf.

Ähnlich sind auch die Befunde für das Deutsche Weidelgras zu deuten. Das Deutsche Weidelgras hob sich an beiden Standorten mit vergleichsweise hohen Nickelgehalten bzw. Nickelabfuhren von einigen anderen Varianten ab. Auch beim Teilindex Spurenelemente ordnet es sich hinter den Ackerbohnen mit positiven Werten ein. Beim Cobalt war der Beitrag des Weidelgrases jedoch gering. Mit 0,034 $\mathrm{mg}$ Co $\mathrm{kg}^{-1} \mathrm{TM}$ am Standort Schoningen im zweiten Versuchsjahr hatte z.B. das Deutsche Weidelgras zwar nur etwa ein Siebtel des Cobaltgehalts 
der Sommerackerbohnen mit 0,254 $\mathrm{mg} \mathrm{Co} \mathrm{kg}^{-1} \mathrm{TM}^{-}$akkumuliert, damit aber immer noch über die elffache Menge als der Mais mit $0,003 \mathrm{mg} \mathrm{kg}^{-1} \mathrm{TM}$. Der daraus errechnete Beitrag zum Teilindex Spurenelemente hatte daher einen günstigeren Wert als anhand der ermittelten, doch eher geringen Cobaltgehalte erwartet wurde. Zusammen mit dem Beitrag des Nickels zum Teilindex Spurenelemente kam schließlich ein vergleichsweise hoher positiver Indexwert für das Deutsche Weidelgras zustande.

Es wird deutlich, dass die Konstruktion des Teilindex Spurenelemente, berechnet aus den Aufnahmen von Cobalt und Nickel, letztendlich keine Aussage über beide Elemente zulässt. Zudem ist es mit dem Ansatz, die Feldfrüchte relativ zueinander zu betrachten nicht möglich, den tatsächlichen Beitrag zur Versorgung des Biogasfermenters mit Cobalt und Nickel durch Input der untersuchten Feldfrüchte abzuschätzen. Wie dargestellt, war z.B. der Cobaltgehalt des Deutschen Weidelgrases dem des Maises vielfach überlegen, was sich auch im Teilindexwert widerspiegelte, aber für die Versorgung des Biogasfermenters ist dies dennoch bei Weitem nicht ausreichend. Diese Einschätzung ist möglich, weil selbst die Ackerbohnen mit wesentlich höheren Cobaltgehalten nicht ausreichen. Zumal selbst die Ackerbohnen mit vergleichsweise hohen Cobaltgehalten bei einer Zumischung von $40 \%$ zu Mais den Cobaltbedarf der Mikroorganismen im Biogasfermenter theoretisch nicht decken können (Fahlbusch et al. 2018). Hier stößt der Ansatz an seine Grenzen.

Würden die beiden Elemente Coablt und Nickel in jeweils einem separaten Teilindex erfasst, wäre bei Betrachtung der einzelnen Teilindexwerte eine klarere Bewertung der Varianten hinsichtlich der Spurenelementaufnahmen möglich. Bei der hier vorgeschlagenen Konstruktion des Gesamtindex IrA durch Addition der Teilindexwerte würden sich jedoch die Werte der beiden Teilindizes Cobalt und Nickel, genau wie bei der Vorgehensweise der gemeinsamen Betrachtung beider Elemente in einem Teilindex, kompensieren. Bei einer möglichen Nutzung des Index IrA als Praxistool müsste dies berücksichtigt werden. Um den tatsächlichen Beitrag der Feldfrüchte zur Spurenelementversorgung des Biogasfermenters mithilfe des Teilinidex Spurenelemente bzw. des Index IrA abschätzen zu können, wäre es möglich, sich an Schwellenwerten für einen optimalen Biogasprozess zu orientieren und alle Varianten, anstatt zur mittleren Spurenelementaufnahme aller Varianten, zu diesem ins Verhältnis zu setzen.

Cobalt ist kein essenzielles Nährelement für höhere Pflanzen, für Mikroorganismen im Biogasbildungsprozess (Demirel \& Scherer 2011) aber auch für Mikroorganismen, die an der $\mathrm{N}_{2}$ Fixierung bei Leguminosen beteiligt sind (Marschner 2012), hingegen schon. Hinweise auf eine positive Wirkung geringer Cobaltmengen bestehen aber ebenfalls für Nicht-Leguminosen durch ein Mitwirken bei der Chlorophyllbildung (Kabata-Pendias 2011). Die Cobaltgehalte im Boden sind abhängig vom Ausgangsmaterial der Bodenbildung. Durch Verwitterung cobalthaltiger Minerale wird Cobalt freigesetzt und kann von den Pflanzen aufgenommen werden (Mengel 1991). Die Pflanzen nehmen Cobalt in Form von $\mathrm{Co}^{2+}$ aus der Bodenlösung auf. Wichtig ist die Konzentration des lons in der Bodenlösung. Insbesondere bei hohen pH-Werten ist Cobalt im Boden fest an Mangan- und Eisenmineralien gebunden und damit nicht pflanzenverfügbar (Kabata-Pendias 2011). Li et al. (2004) fanden zudem heraus, dass der organisch gebundene Cobalt im Boden eine wichtige Quelle für die Cobaltaufnahme von Pflanzen ist. Literaturangaben zu Cobalt-Gesamtgehalten in Böden liegen bei 0,5-70 mg Co kg-1 Boden (Kabata-Pendias 2011). Auch unter Vernachlässigung von Hyperakkumulatoren für Cobalt bewegen sich die Cobalt-Gesamtgehalte in der pflanzlichen Biomasse in einer weiten Spanne. 
Selbst innerhalb einer Art kann es große Unterschiede geben. So wurden z.B. Cobaltgehalte im Weizenkorn zwischen 0,005 und 0,27 mg Co kg-1 TM in Anhängigkeit vom Standort gefunden (Kabata-Pendias 2011). Die Aufnahme des Cobalts über die Wurzel bei höheren Pflanzen erfolgt über passive Diffusion und aktiven Transport (Palit \& Sharma 1994, Kabata-Pendias 2011). Innerhalb der Pflanzen folgt es hauptsächlich dem Transpirationsstrom (Mengel 1991). Laut Palit \& Scharma (1994) gibt es einen Gradienten in der Verteilung des Cobalts in der Pflanze. Der höchste Gehalt liegt demnach in der Wurzel vor, gefolgt von den Blättern, den Samen und zuletzt dem Stängel.

Leguminosen, die unter Cobaltmangelbedingungen angebaut werden und gleichzeitig aufgrund von N-Mangelernährung auf $\mathrm{N}_{2}$-Fixierung angewiesen sind, sind im Wachstum und der Entwicklung im Gegensatz zur gut mit Stickstoff versorgten Kontrolle stark gehemmt (Marschner 2012). Cobalt stellt die Metallkomponente im Cobalamin, auch bekannt als Vitamin $B_{12}$, dar. Cobalamin-haltige Enzyme wurden wiederum in Rhizobien gefunden. Sie sind beteiligt an der Leghämoglobinsynthese, im Stoffwechsel der Rhizobien und bei der Methioninsynthese (Mengel 1991). Durch die Symbiose mit Rhizobien und deren cobalthaltigen Bestandteilen gelangen bei Leguminosen offensichtlich auch im Vergleich zu Nicht-Leguminosen höhere Mengen Cobalt in den Spross und werden dort akkumuliert. So sind zumindest die vergleichsweise hohen Cobaltgehalte im Spross bei Winter- und Sommerackerbohnen der vorliegenden Untersuchung erklärbar. Auch für Wiederkäuer ist die Cobaltversorgung über das Grundfutter wichtig um Vitamin $\mathrm{B}_{12}$-Mangel vorzubeugen. Um dies zu gewährleisten erfolgt auf cobaltarmen Böden eine Düngung mit cobalthaltigen Düngemitteln (Marschner 2012).

Genau wie Cobalt ist auch Nickel essenziell für Mikroorganismen im Biogasbildungsprozess (Demirel \& Scherer 2011). Nickel kann im Gegensatz zu Cobalt aufgrund des Auftretens von Nickelmangelsymptomen als essenzieller Pflanzennährstoff aufgefasst werden. Die Funktion des Nickels liegt dabei vor allem in der Beteiligung am Enzym Urease (Marschner 2012). Besondere Bedeutung hat die Urease bei Harnstoffernährung der Pflanzen aber auch bei Leguminosenarten, bei denen der Stickstoff in Ureidform aus den Knöllchen in den Spross transportiert wird. Nickel wird über passive Diffusion und aktiven Transport (Chatzistathis 2018) in Form von $\mathrm{Ni}^{2+}$ aufgenommen (Mengel 1991). Wie bei der Verfügbarkeit von Cobalt spielt auch beim Nickel der $\mathrm{pH}$-Wert im Boden eine entscheidende Rolle sowie die Konzentration in der Bodenlösung. Mit abnehmendem pH-Wert wird die Adsorption an Bodenpartikel verringert und die Pflanzenverfügbarkeit steigt. Die Gesamtgehalte im Boden liegen abhängig von der Bodenart bzw. vom Ausgangsmaterial der Bodenbildung zwischen 4 und $92 \mathrm{mg} \mathrm{Ni} \mathrm{kg}^{-1}$ Boden (Kabata-Pendias 2011). Laut Marschner (2012) liegt der Nickelgehalt im Aufwuchs zwischen 0,01 und $10 \mathrm{mg} \mathrm{kg}^{-1} \mathrm{TM}$, abhängig vor allem von der Pflanzenart.

In der vorliegenden Untersuchung fielen die Sommer- und Winterackerbohnen auf, die mit

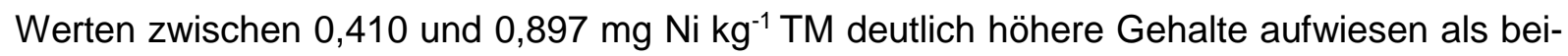
spielweise der Mais mit 0,071 bis $0,144 \mathrm{mg} \mathrm{Ni} \mathrm{kg}^{-1} \mathrm{TM}$. Ackerbohnen weisen Knöllchen des indeterminierten Typs auf, bei denen der fixierte Stickstoff aus den Knöllchen in Amidform und nicht wie bei Sojabohnen mit determinierten Knöllchen als Ureid in den Spross transportiert wird. Daher wird die nickelhaltige Urease vor diesem Hintergrund nicht unbedingt benötigt. Der höhere Nickelgehalt bei den Ackerbohnen im vorliegenden Versuch im Vergleich zu einigen anderen, nicht-legumen Feldfrüchten ist damit also nicht erklärbar. Möglicherweise führte hier die Ansäurung der Rhizosphäre durch die Ackerbohnen zu einer Mobilisierung von Nickel und 
eventuell auch von Cobalt und damit zu einer Förderung der Aufnahme. Solche Mechanismen sind bei Ackerbohnen im Hinblick auf die Phosphorverfügbarmachung bekannt (Li et al. 2007). Auch Phosphat ist wie Cobalt und Nickel bei niedrigen $\mathrm{pH}$-Werten $(<\mathrm{pH} 6)$ besser verfügbar als bei hohen (Gal et al. 2008, Marschner 2012). Li et al. (2007) konnten eine Förderung der Phosphoraufnahme bei Mais zeigen, wenn er im Gemenge mit Ackerbohnen angebaut wurde. Für die Nickel- und Cobaltaufnahme des Gemengepartners Wintertriticale im Gemenge aus Winterackerbohnen und Wintertriticale konnte dies hier jedoch nicht beobachtet werden.

Auffällig sind auch die Unterschiede in den Cobalt- wie auch Nickelgehalten zwischen Sommer- und Winterackerbohnen: Die Sommerackerbohnen an beiden Standorten und in beiden Versuchsjahren wiesen höhere Gehalte auf als die Winterackerbohnen (Tabelle 11). Eine mögliche Erklärung liefert hier die Tatsache, dass es sich bei den Sommer- und Winterackerbohnen um unterschiedliche Genotypen handelt. Auch die verschiedenen Entwicklungsstadien der Varianten bei der Ernte könnten einen Effekt gehabt haben. Da den Sommerackerbohnen eine längere Vegetationszeit zur Verfügung stand als den Winterackerbohnen, stand innen auch eine längere Zeit für den Stoffaustausch im Wurzelraum zur Verfügung. Möglicherweise konnten die Sommerackerbohnen dadurch höhere Cobalt- und Nickelmengen anreichern.

Neben den Ackerbohnen hat in der vorliegenden Untersuchung auch das Deutsche Weidelgras im Vergleich mit den übrigen untersuchten Feldfrüchten eine höhere Nickelakkumulation von bis zu 0,750 $\mathrm{mg} \mathrm{Ni} \mathrm{kg}^{-1}$ TM aufgezeigt. Yang et al. (1997) untersuchten die Nickelaufnahme und Verteilung in den Pflanzen bei Deutschem Weidelgras und Mais unter Steigerung des Nickelangebots im Boden. Deutsches Weidelgras akkumulierte wesentlich mehr Nickel im Spross als Mais. In den Wurzeln hingehen unterschieden sich bei mittlerem Nickelangebot (unterhalb der auf die Wurzel toxisch wirkenden Grenze) die Gehalte zwischen den Pflanzenarten nicht. Das Deutsche Weidelgras hat aber die 7-8fache Menge im Vergleich zu Mais in den Spross transportiert. Der Mechanismus, Nickel nicht in hohen Mengen aus den Wurzeln in den Spross zu transportieren, macht Mais offenbar toleranter gegenüber hohen Nickelgehalten im Boden als Deutsches Weidelgras. Weidelgras hingegen ist sensitiver gegenüber hohen Nickelgehalten. Obwohl die Nickelgehalte in der vorliegenden Untersuchung sowohl im Boden als auch in den Pflanzen wesentlich geringer waren als bei Yang et al. (1997), werden die Unterschiede, möglicherweise beruhend auf unterschiedlichen Mechanismen der Nickelverteilung in den Pflanzen, zwischen den Arten jedoch auch hier deutlich.

Die Erwartung, durch den Anbau von Gemengen aus einer spurenelementakkumulierenden und einer ertragreichen Feldfrucht die Qualität des Gemenges hinsichtlich der Spurenelementgehalte zu verbessern, wurde zumindest beim Gemenge aus Winterackerbohnen und Wintertriticale erreicht. Im Durchschnitt über die beiden Standorte und Versuchsjahre zeigte das Gemenge einen um fast 8-fach höheren Cobaltgehalt sowie einen um fast 3-fach höheren Nickelgehalt auf als die Wintertriticale in Reinsaat (Tabelle 11). Dies macht sich auch beim Teilindex Spurenelemente dieser Varianten bemerkbar. Während die Wintertriticale in Reinsaat hier Indexwerte im deutlich negativen Bereich aufweist, sind sie beim Gemenge stets im positiven Bereich - z.T. auch deutlich (Tabelle 19, Tabelle 20). Beim Gemenge aus Amarant und Mais hingegen wurden im Vergleich zur Mais-Reinsaat keine höheren Spurenelementgehalte $(\mathrm{Co}, \mathrm{Ni})$ gefunden. Zum einen hatte der Amarant im Vergleich zu den Winterackerbohnen 
auch selbst wesentlich geringere Gehalte an Cobalt und Nickel, sodass hier eine Qualitätsverbesserung auch nur schwer möglich war, zum anderen war der Anteil des Amarants am Gemenge mit Mais auch wesentlich geringer als der der Winterackerbohnen als Gemengepartner von Wintertriticale (Daten nicht dargestellt). Die Werte für den Teilindex Spurenelemente des Gemenges aus Amarant und Mais unterschieden sich demzufolge auch nicht von dem der Mais-Reinsaat.

Auffällig sind die Unterschiede in den Gehalten von Cobalt und Nickel bei den untersuchten Feldfrüchten in Abhängigkeit vom Standort (Tabelle 11). Feldfrüchte, die am Standort Reinshof aufwuchsen, hatten überwiegend höhere Nickelgehalte als Feldfüchte am Standort Schoningen. So wiesen z.B. die Winterackerbohnen 0,630 und 0,621 $\mathrm{mg} \mathrm{Ni} \mathrm{kg}^{-1} \mathrm{TM}$ im ersten bzW. zweiten Versuchsjahr am Standort Reinshof auf, am Standort Schoningen hingegen nur 0,287 bzw. 0,410 mg Ni kg${ }^{-1}$. Höhere Nickelgehalte wurden entsprechend den Pflanzengehalten auch im Boden am Standort Reinshof mit 16,5 $\mathrm{mg} \mathrm{Ni} \mathrm{kg}^{-1}$ Boden im Vergleich zum Standort Schoningen mit 11,8 $\mathrm{mg} \mathrm{Ni} \mathrm{kg}^{-1}$ Boden gefunden. Der pH-Wert am Standort Schoningen lag etwas unter dem am Standort Reinshof. Dies müsste theoretisch auch die Nickelaufnahme am Standort Schoningen begünstigen. Der höhere Ni-Gehalt im Boden am Standort Reinshof scheint also für die Aufnahme ausschlaggebend gewesen zu sein.

Bei den Cobaltgehalten trifft genau das Gegenteil zu: Am Standort Schoningen konnten nahezu alle untersuchten Feldfrüchte höhere Cobaltgehalte erreichen als am Standort Reinshof. Wie anhand des Korrelationskoeffizienten von $r=-0,521 \quad(p<0,001$, Tabelle 18) abgeleitet werden kann, besteht ein mittlerer negativer Zusammenhang zwischen dem Cobaltgehalt und dem Trockenmasseertrag: Je höher der Cobaltgehalt umso geringer der Trockenmasseertrag. Dies weist entweder auf einen Verdünnungseffekt des Cobalts in der Biomasse hin oder auf eine Standortabhängigkeit, da auch mit dem Standort Unterschiede in den Trockenmasseerträgen einhergingen. Da das Deutsche Weidelgras als einzige Variante aber am Standort Schoningen gleichauf mit den Trockenmasseerträgen am Standort Reinshof war und dennoch höhere Cobaltgehalte aufwies, spricht dies eher für eine Standortabhängigkeit. Einen Zusammenhang zwischen den Cobaltgehalten und den Trockenmasseerträgen konnten auch Li et al. (2004) bei Weidelgras nicht bestätigen. An den beiden Standorten der vorliegenden Untersuchung unterschieden sich die Cobaltgehalte im Boden, ermittelt im Säuretotalaufschluss, etwas. So waren am Standort Reinshof 7,1 und am Standort Schoningen 8,1 mg Co kg-1 Boden vorhanden (Fahlbusch et al. 2018).

Wie aber Li et al. (2004) herausfanden, wird die Cobaltaufnahme auch stark von den Mangangehalten im Boden beeinflusst: Je höher der Mangangehalt im Boden war, umso geringer war die Aufnahme von Cobalt in die Biomasse von Weidelgras. Als Grund wird die, wie bereits angesprochen, spezifische Sorption von Cobalt an der Oberfläche von Mangan- und Eisenoxiden und der damit zusammenhängenden geringen Pflanzenverfügbarkeit angegeben. Hinsichtlich der Mangangehalte im Boden gab es auch in der vorliegenden Untersuchung deutlichere Unterschiede zwischen den Standorten als bei den anderen Spurenelementen. Mit 704 mg Mn kg-1 Boden am Standort Reinshof (Fahlbusch et al. 2018) liegt der Mangangehalt hier im von Li et al. (2004) identifizierten Bereich starker Hemmung der Cobaltaufnahme. Mit 479 $\mathrm{mg} \mathrm{Mn} \mathrm{kg}{ }^{-1}$ Boden war der Mangangehalt im Boden am Standort Schoningen (Fahlbusch et al. 2018) deutlich unter dem des Standorts Reinshof. In der Untersuchung von Li et al. (2004) stieg die Cobaltaufnahme beim Weidelgras in diesem Bereich tendenziell an. Möglicherweise 
könnte auch im vorliegenden Versuch ein Zusammenhang zwischen der Cobaltaufnahme der Pflanzen und den Mangangehalten im Boden bestanden haben. Da zudem die Cobaltaufnahme stark abhängig vom pH-Wert ist, lässt auch dies Unterschiede zwischen den Standorten und dabei höhere Aufnahmen am Standort Schoningen als am Standort Reinshof erwarten.

Die Jahresunterschiede bei den Cobaltgehalten (Tabelle 11) am Standort Schoningen beim Gemenge aus Winterackerbohnen und Wintertriticale sowie der mehrjährigen Blühmischung lassen sich möglicherweise durch unterschiedliche Zusammensetzungen des Aufwuchses erklären. Bei der mehrjährigen Blühmischung dominierten zum einen, wie schon erwähnt, je nach Standjahr unterschiedliche Arten und zum anderen sind auch Unterschiede in der Probenzusammensetzung zwischen den Jahren bei vielfältigen Beständen nicht auszuschließen. Für eine inhomogene Probenzusammensetzung spricht auch die vergleichsweise hohe Standardabweichung im zweiten Versuchsjahr. Interessant sind die Jahresunterschiede in den Cobaltgehalten bei der Winterackerbohnen-Reinsaat sowie dem Gemenge aus Winterackerbohnen und Wintertriticale am Standort Schoningen. Tendenziell war dieser Jahreseffekt bis auf die Sommerackerbohnen auch bei den anderen Varianten zu beobachten, jedoch meist weniger stark ausgeprägt. Wenn, wie bereits erwähnt, die Cobaltakkumulation bei Leguminosen stark mit der Rhizobiensymbiose zusammenhängt, könnte da möglicherweise ein Grund liegen. Trotz eines sogar höheren Trockenmasseertrags der Winterackerbohnen im ersten im Vergleich zum zweiten Versuchsjahr mit 94 bzw. 85 dt TM ha-1 (Abbildung 7), war die N-Akkumulation im Spross mit $206 \mathrm{~kg} \mathrm{~N} \mathrm{ha}^{-1}$ im ersten Versuchsjahr geringer als im zweiten mit 256 $\mathrm{kg} \mathrm{N} \mathrm{ha}^{-1}$ (Abbildung 11). Beim Gemenge aus Winterackerbohnen und Wintertriticale war dies in abgeschwächter Form ebenfalls zu beobachten. Entweder war im ersten Versuchsjahr die Symbiose der Winterackerbohnen mit den Rhizobien nicht besonders gut ausgeprägt, was auch zu weniger Cobalt im Spross führte, oder im zweiten Versuchsjahr lebten die Pflanzen unter Bedingungen, bei denen Luxuskonsum hinsichtlich der Stickstoffaufnahme möglich war und die unterschiedlichen Cobaltaufnahmen eine andere Ursache hatten. Gegen Letzteres spricht allerdings, dass die zur Verfügung gestandenen Nmin-Mengen im Boden bei Winterackerbohnen im zweiten Versuchsjahr geringer waren als im ersten (Abbildung 18, Abbildung 19) und die $\mathrm{N}$-Düngemenge in beiden Versuchsjahren gleich war. Die $\mathrm{N}_{2}$-Fixierleistung hängt u.a. von den im Boden zur Verfügung stehenden N-Mengen ab: Hohe N-Mengen hemmen die $\mathrm{N}_{2}$-Fixierung, geringerer N-Mengen fördern sie (Jost 2003). Die geringe N-Verfügbarkeit könnte im zweiten Versuchsjahr des vorliegenden Versuchs die $\mathrm{N}_{2}$-Fixierung gefördert haben wobei möglicherweise gleichzeitig höhere Cobaltmengen in den Spross gelangen konnten als im ersten.

Neuere Untersuchungen haben neben der Identifizierung von Cobalt und Nickel als wichtige Spurenelemente um den Biogasprozess stabil zu halten, gezeigt, dass auch Natrium ein in Mangel kommendes Element sein könnte. In Mais sind, Pasold (2020) zufolge, im Vergleich mit anderen Feldfüchten nur sehr geringe Natriummengen enthalten. Auch ein durch hauptsächlichen Maisinput hervorgerufener Mangel dieses Elements könnte zu Instabilität des Biogasprozesses führen. Die könnte in späteren Untersuchungen berücksichtigt werden. 


\subsubsection{Teilindex Ökonomische Aspekte}

Neben dem Trockenmasseertrag der oberirdischen Biomasse und dem Methanflächenertrag ist für die Bewertung der Leistungsfähigkeit von Energiepflanzen für die Biogaserzeugung, wie für Marktfrüchte auch, die Wirtschaftlichkeit der Feldfrüchte von großer Bedeutung. Die ökonomische Bewertung, abgebildet mithilfe von Deckungsbeiträgen, setzt sich aus der variablen Leistung und den variablen Kosten zusammen. Die Leistung ist dabei stark abhängig vom Trockenmasseertrag, der ebenfalls als Teilindex in IrA eingeht. Wichtig für die Berechnung der Deckungsbeiträge sind aber die Kosten, die mit dem Anbau der Feldfrüchte einhergehen. Das bedeutet, dass die Trockenmasseerträge zwar im Teilindex Ökonomische Aspekte indirekt enthalten sind, aber die Varianten eine z.T. deutlich unterschiedliche Kostenstruktur haben, sodass nicht unbedingt direkt von den Trockenmasseerträgen auf die Wirtschaftlichkeit des Anbaus geschlossen werden kann.

Die Leistung setzt sich zusammen aus dem Erlös, den die Biomasse erbringt sowie dem Gärrestwert. Der Gärrest wurde nicht, wie in der Vorgehensweise von z.B. Auerbacher et al. (2018), als ein geschlossener Gärrestkreislauf angenommen, bei dem der anfallende Gärrest mit der Gärrestdüngung verrechnet wird, sondern unter Berücksichtigung der Abbauraten die anfallende Menge monetär als Leistung angerechnet. Insbesondere die Winter- und Sommerackerbohnen akkumulierten viel Stickstoff in der oberirdischen Biomasse (Abbildung 10, Abbildung 11). In Abhängigkeit vom Anteil der Beimischung dieser Varianten in den Fermenter, ist mit einem stickstoffreichen Gärrest zu rechnen. Die Ackerbohnen selbst bedürfen dieser hohen N-Düngemengen nicht. Zu prüfen wäre auch, ob es bei einem Gärrest aus Ackerbohnen bei praxisüblicher Gärrestdüngung von Energiepflanzen zu Konflikten mit der Düngeverordnung kommt. Denkbar wäre auch eine vermehrte organische Düngung von Marktfrüchten mit den im Energiepflanzenanbau nicht benötigten stickstoffreichen Gärresten, was hier eine Einsparung an N-Düngemitteln nach sich ziehen würde.

Die Kosten variieren zwischen den Versuchsjahren und Standorten etwas, da immer die tatsächlich angefallenen Arbeiten, Aufwandmengen an Düngemitteln usw. in die Kalkulation einbezogen wurden (Tabelle 17). Diese waren in beiden Versuchsjahren bzw. an beiden Standorten nicht gleich. Unberücksichtigt in der Kalkulation blieben aber eventuell höher ausfallende Kosten bei Biomasse mit niedrigen TS-Gehalten im Vergleich zu optimalen. Hier können steigende Kosten z.B. durch höhere Aufwendungen für den Transport und zusätzlichen Bedarf an Siliermitteln entstehen.

Wie stark die Kosten den Deckungsbeitrag und damit auch den Teilindex Ökonomische Aspekte bestimmen, wird an den Varianten des Zweikulturnutzungssystems deutlich. Diese Varianten generierten eine mit Abstand höhere Leistung im Vergleich zu Mais als Hauptfrucht, blieben aufgrund der höheren Kosten durch den Anbau von zwei Kulturpflanzen in einer Vegetationsperiode jedoch beim Deckungsbeitrag sowie der ökonomischen Bewertung hinter dem Mais zurück (Tabelle 17, Tabelle 19, Tabelle 20). Deckungsbeiträge zwischen 900 und $1300 €$ ha $^{-1}$ bei den Varianten des Zweikulturnutzungssystems am Standort Reinshof sind dennoch vergleichsweise hoch. Der Mais als Hauptfrucht erzielte mit 1220 und $1340 €$ ha $^{-1}$ an diesem Standort höhere und stabilere Deckungsbeiträge. Am Standort Schoningen waren die Deckungsbeiträge insgesamt auf einem niedrigeren Niveau. Die beim Anbau der Varianten 
des Zweikulturnutzungssystems entstandenen Kosten waren an beiden Standorten vergleichbar, die Trockenmasseerträge jedoch nicht. So wurde am Standort Schoningen die erzielbare Leistung durch die Kosten noch deutlicher aufgezehrt als am Standort Reinshof. Im zweiten Versuchsjahr führte dies hier sogar zu überwiegend negativen Werten für den Teilindex Ökonomische Aspekte. Standort- und Jahresabhängigkeit bei der ökonomischen Bewertung beim Zweikulturnutzungssystem aus Roggen gefolgt von Mais und Mais als Hauptfrucht zeigten auch Toews et al. (2008). Bei diesen Autoren konnte der Gewinn durch das Zweikulturnutzungssystem an zwei der sieben einbezogenen Standorte in beiden Untersuchungsjahren gegenüber Mais als Hauptfrucht gesteigert werden. An den anderen Standorten war das Ergebnis nicht so eindeutig: In einem Versuchsjahr brachte der Mais als Hauptfrucht ein besseres Ergebnis, im anderen das Zweikulturnutzungssystem oder der Mais als Hauptfrucht übertraf dieses in beiden Jahren. Auch der von Toews et al. (2008) ermittelte, tatsächliche Gewinn mit 409 bis $1148 €$ ha $^{-1}$ übersteigt die Ergebnisse des vorliegenden Versuchs am Standort Schoningen z.T. deutlich. Bei den im vorliegenden Versuch an diesem Standort erzielten Deckungsbeiträgen mit 303 bis $944 €$ ha $^{-1}$ müssen für die Gewinnermittlung noch die festen Kosten abgezogen werden (Dabbert \& Braun 2006). Der tatsächliche Gewinn des Landwirts dürfte also noch um einiges geringer sein.

Ein, insbesondere an den Standort Schoningen, besser angepasstes Anbausystem scheint das Deutsche Weidelgras zu sein. Mit einem Deckungsbeitrag von $994 €$ ha $^{-1}$ überstieg es hier alle anderen Varianten, auch den Mais als Hauptfrucht mit $824 € \mathrm{ha}^{-1}$ als zweitwirtschaftlichste Variante. Somit zeichnet sich das Deutsche Weidelgras am Standort Schoningen auch mit einem hohen positiven Wert für den Teilindex Ökonomische Aspekte aus. Am Standort Reinshof ordnete es sich zumindest im ersten Versuchsjahr bei der ökonomischen Bewertung unter den besten Varianten ein. Im Vergleich mit den übrigen hochleistenden Varianten waren die anfallenden Kosten, bei Annahme einer vierjährigen Nutzung als Dauerkultur mit drei Schnitten pro Jahr, beim Deutschen Weidelgras gering.

Ebenfalls vergleichsweise geringe Kosten im Verhältnis zur Leistung zeigte die Durchwachsene Silphie als Dauerkultur. Mit einem Deckungsbeitrag von $1217 €$ ha $^{-1}$ am Standort Reinshof erzielte diese Variante einen mit den Varianten des Zweikulturnutzungssystems vergleichbar hohen Wert beim Teilindex Ökonomische Aspekte. Bei der Kostenkalkulation wurde angenommen, dass die Silphie, wie im vorliegenden Versuch durchgeführt, über Pflanzung etabliert wurde. Die Nutzungsdauer wurde auf 15 Jahre angesetzt. Das Jahr, in dem der Bestand etabliert wird, also Kosten entstehen, aber keine Leistung erzielt wird, sowie das letzte Jahr, im dem der Umbruch zusätzliche Kosten verursacht, wurden auf die Nutzungsjahre umgelegt. Damit belaufen sich die Kosten in jedem der 15 Nutzungsjahre auf $528 €$ ha $^{-1}$ am Standort Reinshof und $492 €$ ha $^{-1}$ am Standort Schoningen. Auf ähnliche jährliche Kosten kommen bei gleicher Nutzungsdauer auch Biertümpfel \& Conrad (2013) bei Etablierung mittels Pflanzung. Ein großer Kostenfaktor ist dabei die Pflanzung selbst mit etwa $5000 €$ ha $^{-1}$ (Biertümpfel \& Conrad 2013). Bei der Etablierung über Aussaat in Form von Blanksaat kommen Biertümpfel \& Conrad (2013) bei gleicher Nutzungsdauer bereits auf deutlich geringere jährliche Kosten von etwa $280 €$ ha $^{-1}$. Für die Etablierung mittels Aussaat wurden dabei $60 \%$ der Kosten der Pflanzvariante, also etwa $3000 € \mathrm{ha}^{-1}$, ermittelt. Bei Annahme der Etablierung über Aussaat im hier vorliegenden Versuch und den von Biertümpfel \& Conrad (2013) ermittelten Kosten, wären am Standort Reinshof Deckungsbeiträge von $1465 €$ ha $^{-1}$ möglich gewesen. Damit wäre die 
Durchwachsene Silphie hier die ökonomisch anbauwürdigste Variante. Ob bei Aussaat im ersten Nutzungsjahr die gleichen Trockenmasseerträge erzielt worden wären, bliebe zu prüfen. Bei Annahme des gleichen Szenarios am Standort Schoningen käme es zwar auch zu einer Verbesserung der Deckungsbeträge bei der Durchwachsenen Silphie, das Deutsche Weidelgras wäre aber dennoch bei Weitem nicht eingeholt.

Eine andere, noch weiter die ökonomische Bewertung verbessernde Methode bei der Bestandsetablierung der Durchwachsenen Silphie ist die Aussaat als Untersaat in Mais. Im ersten Jahr wird dabei der Mais, unter dem die Durchwachsene Silphie aufläuft und eine Rosette bildet, geerntet. So fällt das bei Pflanzung oder Aussaat als Blanksaat vorhandene unproduktive erste Jahr weg und die jährlichen Deckungsbeiträge steigen weiter an (Kahnt-Ralle 2017).

Seit 2018 ist die Durchwachsene Silphie im Rahmen des im Jahr 2015 eingeführten Greenings der Gemeinsamen Agrarpolitik (GAP) anrechenbar. Die Greeningauflagen sehen vor, $5 \%$ der Ackerfläche als ökologische Vorrangfläche vorzuhalten und bestimmte Vorgaben einzuhalten. Der Anbau der Durchwachsenen Silphie kann hier mit dem Faktor 0,7 angesetzt werden (BMEL 2018). Das bedeutet, dass 1 ha Durchwachsene Silphie 0,7 ha ökologischer Vorrangfläche entsprechen. So kann auch auf einer ökologischen Vorrangfläche produktiv gewirtschaftet werden, was bei anderen wählbaren Maßnahmen nicht immer der Fall ist. Es ist z.B. beim Anbau von Leguminosen als ökologische Vorrangfläche die Anwendung von Pflanzenschutzmitteln untersagt (BMEL 2018), was den Anbau u.U. schwierig gestalten kann. Bei der Durchwachsenen Silphie ist die Anwendung von Pflanzenschutzmitteln im ersten Jahr zugelassen, in den Folgejahren nicht (LWK Nordrhein-Westfalen 2020), was dann jedoch auch nicht mehr erforderlich ist (Kahnt-Ralle 2017). Zudem ist auch eine organische Düngung zulässig (LWK Nordrhein-Westfalen 2020). So kann sich auch der Aspekt des Greenings positiv auf die ökonomische Bewertung der Durchwachsenen Silphie auswirken und die Vorzüglichkeit aus dieser Sicht steigern.

Auch bei der mehrjährigen Blühmischung gibt es, vergleichbar mit der Bestandsetablierung der Durchwachsenen Silphie, die Möglichkeit, die Aussaat als Untersaat in Mais vorzunehmen, was von Cossel et al. (2019) zufolge aus ökonomischer Sicht eine Verbesserung gegenüber der Aussaat als Blanksaat darstellt. Dabei laufen die mehrjährigen Arten der Blühmischung im Mais auf, können sich nach der Ernte des Maises entwickeln und bilden in den Folgejahren den Bestand. Gerade die Blühmischungen schnitten in der hier vorliegenden Untersuchung im Vergleich mit den anderen Feldfrüchten bei der ökonomischen Bewertung schlecht ab. Sie zeigten die mitunter größten negativen Werte beim Teilindex Ökonomische Aspekte. Eine Verbesserung der Wirtschaftlichkeit, etwa mithilfe von optimierten Etablierungsmöglichkeiten, ist vonnöten um mit anderen Varianten konkurrieren zu können. Ebenfalls deutlich negativ sind die Werte für den Teilindex Ökonomische Aspekte bei den Sommerackerbohnen. Die vergleichsweise hohen Kosten der Sommerackerbohnen sind v.a. bedingt durch die hohen Saatgutkosten. Der bereits angesprochene Anbau einer Sommerzwischenfrucht zur Biogasnutzung nach den Sommerackerbohnen könnte wahrscheinlich zur Abmilderung dieser Schwachstelle beitragen. Allerdings könnten die Kosten, wie bei den Varianten des Zweikulturnutzungssystems beobachtet, aufgrund der mehrfachen Aussaat stärker steigen als die Leistungen durch die zusätzliche Biomasse der Zwischenfrucht. 
Unberücksichtigt bei der ökonomischen Bewertung bleibt aber der ökonomische Vorfruchteffekt, der gerade bei den Ackerbohnen höher sein dürfte als bei anderen Varianten der Untersuchung. Völkel \& Schmidtke (2019) geben für Ackerbohnen einen N-Saldo von +49 kg N/ha an. Dieser Wert kann ein grober Anhaltspunkt für die stickstoffbedingte Vorfruchtwirkung der Ackerbohnen sein. Die Angaben beziehen sich allerdings auf die Körnernutzung der Ackerbohnen und sind daher nicht ohne Weiteres auf die Nutzung als Ganzpflanzensilage bei Ernte in einem früheren Entwicklungsstadium als zur Kornreife übertragbar. Jost (2003) erfasste die N-Flächenbilanz eines (Sommer-) Ackerbohnen-Hafer-Gemenges zur Nutzung als Ganzpflanzensilage. Selbst bei diesem Gemenge lag die N-Flächenbilanz im Mittel von zwölf Umwelten (vier Standorte, drei Versuchsjahre) bei $+16,6 \mathrm{~kg} \mathrm{~N}^{-1}$ und bei Berücksichtigung der N-Rhizodeposition sogar bei $+32,4 \mathrm{~kg} \mathrm{~N}^{-1}$. Eine möglicherweise ökonomisch interessante Alternative zu Varianten zur Biogaserzeugung nach Sommerackerbohnen könnte auch, wie bereits angesprochen, der Winterraps zur Kornnutzung sein, der die gute Vorfruchtwirkung gerade bei den Restriktionen in der N-Düngung (Hogrefe 2020) wahrscheinlich gut nutzen kann. Die Bewertung der ökonomischen Vorfruchtwirkungen könnte durch einen möglichen Mehrertrag des Rapses gegenüber dem Anbau ohne Sommerackerbohnen-Vorfrucht berechnet werden.

\subsubsection{Teilindex Soziale Aspekte}

Der Teilindex Soziale Aspekte soll die Akzeptanz der untersuchten Pflanzenvarianten als Energiepflanzen für die Biogaserzeugung in der Bevölkerung abbilden. Die zur Datengenerierung durchgeführte Umfrage war so angelegt, dass die Meinungsbildung anhand der äußeren Erscheinung der Pflanzenvarianten erfolgen sollte und weniger anhand ihrer ökologischen oder ökonomischen Eigenschaften. Damit sollte angeknüpft werden an die in der öffentlichen Wahrnehmung mit der Entwicklung des Biogassektors einhergehenden Auswirkungen auf das Landschaftsbild hin zur „Vermaisung der Landschaft“. Die Landschaft hat in der Gesellschaft einen großen Stellenwert, z.B. durch ihre Freizeitfunktion. Dazu kommen nicht zuletzt die Auswirkungen auf die Gesundheit und das Wohlbefinden der Menschen (Bruns \& Münderlein 2018). Deshalb sollte bei der umfassenden Bewertung von Feldfrüchten für die Biogaserzeugung, die zumindest regional einen größeren Flächenumfang einnehmen können, die Akzeptanz nicht unberücksichtigt bleiben. Sogar im Bundesnaturschutzgesetz ist die Erhaltung u.a. der Schönheit und des Erholungswertes der Natur und der Landschaft festgeschrieben (BNatSchG 2009).

Dass es in Deutschland auch tatsächlich zu einer Flächenzunahme des Maises gekommen ist, zeigt die Entwicklung der Maisanbaufläche in den letzten Jahren, insbesondere in der Zeit seit dem Erneuerbare-Energien-Gesetz aus dem Jahr 2004, in dem ein Bonus für die Stromerzeugung aus nachwachsenden Rohstoffen eingeführt wurde (von Bredow 2010). So ist die Anbaufläche von Silomais in den letzten 15 Jahren um über eine Million Hektar angestiegen von 1248000 ha im Jahr 2004 (Statistisches Bundesamt 2005) auf 2223000 ha im Jahr 2019 (Statistisches Bundesamt 2020). Um dieser Entwicklung zu begegnen wurde schließlich der sogenannte Maisdeckel eingeführt, der den Input von Mais als Substrat begrenzt (Herbes et al. 2014). Teilweise konzentriert sich der Maisanbau stark auf bestimmte Regionen mit z.B. etwa 50 \% der Ackerfläche in der Region Weser-Ems im Jahr 2018 (Landesamt für Statistik Niedersachsen 2019). Hier ist der Mais wahrscheinlich stark landschaftsprägend. In anderen Regionen Deutschlands stellt der Mais eher eine Erweiterung der Fruchtfolge als eine Verengung dar (Karpenstein-Machan \& Weber 2010). 
Seit dem vermehrten Anbau von Mais als Energiepflanze für die Biogaserzeugung werden Methoden entwickelt, um die Auswirkungen auf das Landschaftsbild und die Erholungsfunktion der Landschaft untersuchen bzw. bewerten zu können (Wiehe et al. 2009, Huth et al. 2019). In der vorliegenden Untersuchung sollte durch den Teilindex Soziale Aspekte aufgezeigt werden, welche Feldfrüchte aus Sicht der Bevölkerung aufgrund ihrer Erscheinung im Vergleich zu anderen Feldfrüchten besonders befürwortet werden. Durch diese Konzeption könnte hier ein Beitrag geleistet werden, das Landschaftsbild zu bewerten. Neben der Einbeziehung des Teilindex Soziale Aspekte in den Index IrA, als umfassendes Bewertungsinstrument von Feldfrüchten für die Energieerzeugung, wäre denkbar, dass bei einem Genehmigungsverfahren zum Bau einer Biogasanlage IrA die Grundlage für die Erteilung von Auflagen darstellen könnte, bestimmte Feldfrüchte und zwar solche, die von der Bevölkerung akzeptiert werden, in bestimmten Umfängen anzubauen.

Wie zu erwarten, zeigte der Mais, sichtbar am deutlich negativen Indexwert beim Teilindex Soziale Aspekte, bei den Umfrageteilnehmern die geringste Zustimmung (Abbildung 22, Tabelle 21). Aber immerhin $36 \%$ der Befragten befürworteten den Mais und weitere $22 \%$ waren sich unschlüssig. Huth et al. (2019) fanden in einer Untersuchung, in der die Befragten verschiedene Landschaftsbilder, u.a. mit viel Mais-, mit vereinzelt Mais- und ohne Maisanbau, bewerten sollten, heraus, dass Landschaften mit vereinzeltem Maisanbau genauso bewertet werden wie Landschaften ohne Maisanbau. Landschaften mit viel Mais hingegen erhielten diesen Autoren zufolge deutlich weniger Zustimmung. In der hier vorliegenden Untersuchung wurde der Mais interessanterweise von den Ablehnenden nicht aufgrund seiner optischen Wirkung abgelehnt, zumal danach gefragt wurde, sondern überwiegend, weil er als ökologisch nachteilig angesehen wird (Tabelle 16). Diese Einschätzung würde sich möglicherweise relativieren, wenn Mais anstatt landschaftsdominierend, nur vereinzelt angebaut würde. Offenbar ist es aber insgesamt schwierig, eine Meinungsbildung allein anhand des optischen Eindrucks, ohne eine subjektive Einschätzung anderer Kenngrößen, zu erfassen. Vielmehr soll die ökologische Wirkung der Feldfrüchte im Index IrA mithilfe von objektiven Kriterien über andere Teilindizes einbezogen werden. Möglicherweise hängt dies aber mit der Stichprobe zusammen, denn immerhin $68 \%$ der Befragten gaben an, beruflich mit der Natur verbunden zu sein (Tabelle 5). Vor diesem Hintergrund ist sicherlich auch die Einschätzung des Amarants zu werten. Für den Amarant ergab sich ähnlich dem Mais, ein deutlich negativer Wert beim Teilindex Soziale Aspekte. Die von etwa $30 \%$ der Befragten angegebene Begründung, der Amarant sei hier nicht heimisch, weist schon auf ein gewisses Expertenwissen hin. Weiteren $30 \%$ gefällt der Amarant aber. Auch das Gemenge aus Amarant und Mais kann die Akzeptanz der Bevölkerung gegenüber den Reinsaaten nicht wesentlich steigern, obwohl $40 \%$ der Befragten - und damit mehr als bei der Amarant-Reinsaat - angaben, ihnen gefalle das Gemenge (Tabelle 16).

Bei der von Wiehe et al. (2009) vorgeschlagenen Methode zur Bewertung der Auswirkungen des Energiepflanzenanbaus auf das Landschaftsbild und dabei u.a. auf die Erholungsfunktion erfolgt die Bewertung der Feldfrüchte anhand verschiedener Indikatoren: Das sind die Bodenbedeckung, die Bestandeshöhe und der Blühaspekt. Die Bevölkerung selbst, z.B. durch eine Umfrage, wird nicht einbezogen. Als negativ werden Feldfrüchte bewertet, bei denen der Boden lange Zeit unbedeckt ist, die Bestandeshöhen bis oberhalb der Augenhöhe reichen und eine unauffällige Blüte vorhanden ist. 
Die Wichtigkeit der Bestandeshöhe ist auch in der hier vorliegenden Untersuchung von den Umfrageteilnehmern herausgestellt worden. So empfanden ein Viertel der Befragten die Durchwachsene Silphie als zu hoch. Trotz der sonst positiven Bewertung hat diese Variante nur einen Wert beim Teilindex Soziale Aspekte von nahe Null erhalten, hebt sich also nicht besonders von den anderen Varianten ab. Hier hatte die hohe Bestandeshöhe im Vergleich zur Blüheigenschaft ein wohl stärkeres Gewicht. Dass auch dem Blühaspekt - zumindest bei moderater Bestandeshöhe - eine besondere Bedeutung bei der Akzeptanz von Feldfrüchten als Energiepflanzen zukommt, zeigen die deutlich positiven Werte für den Teilindex Soziale Aspekte bei der ein- und mehrjährigen Blühmischung. Das Blühangebot dieser Varianten wird dabei sowohl als ansehnlich als auch ökologisch vorteilhaft abgesehen. Auch der Wickroggen geht in diese Richtung. Schüpbach et al. (2009) konnten zeigen, dass die Bewertung von blühenden Beständen zudem abhängig ist von der Vielfalt an Blütenfarben, wobei bunt blühende Bestände besser bewertet werden als einfarbig blühende. Dies könnte neben der hohen Bestandeshöhe die nur mittlere Akzeptanz der ausschließlich gelb blühenden Durchwachsenen Silphie im Vergleich zur hohen Akzeptanz der bunt blühenden Blühmischungen in der vorliegenden Untersuchung erklären.

Die untersuchten Feldfrüchte wurden in der Umfrage anhand von Fotos der Parzellenversuche gezeigt. So sahen die Umfrageteilnehmer nur kleine Ausschnitte der Bestände in Nahaufnahme und mussten sich vorstellen, wie eine Landschaft mit größerem Flächenumfang dieser Feldfrüchte wirken würde. Zudem zeigten die Fotos lediglich eine Momentaufnahme der Pflanzen in dem meist, nach eigener Einschätzung, attraktivsten Entwicklungsstadium. In einer Untersuchung zur ästhetischen Bewertung landwirtschaftlicher Feldfrüchte in der Schweiz zeigten Schüpbach et al. (2009) in ihrer Umfrage Fotos zum jahreszeitlichen Verlauf der Feldfrüchte, um alle charakteristischen Entwicklungsstadien in die Bewertung einfließen zu lassen. Wie die Autoren feststellen konnten, wurden die Entwicklungsstadien, in denen viel braun zu sehen war, z.B. die Zeit der Aussaat und der Jugendentwicklung des Maises oder ein frisch gemähter Grasbestand, deutlich negativer bewertet als Entwicklungsstadien der gleichen Kultur mit grünem Erscheinungsbild. Dies bestätigt den von Wiehe et al. (2009) genutzten Indikator, die Zeit mit sichtbarem braunen Boden. Ein solches Vorgehen hätte in der vorliegenden Untersuchung möglicherweise gerade bei den Varianten des Zweikulturnutzungssystems die Bewertung negativ beeinflusst. Bei diesen Varianten wurden die Erstfüchte, nicht aber der Mais als Zweitfrucht, in der Umfrage gezeigt. Das Anbausystem wurde nur beschrieben. Die Erstfrüchte mit Ernte Ende Mai oder Anfang Juni bestimmen dabei das Erscheinungsbild nur vergleichsweise kurze Zeit. Zu einer positiveren Bewertung hingegen wäre es vermutlich bei der Durchwachsenen Silphie gekommen. Diese Variante ist durch ihre Eigenschaft als Dauerkultur nach Wiederaustrieb nach der Ernte (Dauber et al. 2016) grün, wenn bei anderen Kulturen unbedeckter Boden zu sehen ist.

Einen Teilindexwert nahe Null zeigt auch das Deutsche Weidelgras. Auffällig ist hier jedoch der Anteil von $20 \%$ der Befragten, die das Deutsche Weidelgras ablehnen. Dabei bewerten nur etwa $5 \%$ der Befragten diese Variante als unansehnlich, jedoch $20 \%$ als ökologisch nachteilig. Als Argumente wurden z.B. genannt, dass es sich beim Weidelgras um eine intensiv geführte Monokultur handele oder dass dieses Anbausystem zur Artenverarmung beitrage. Wenn auch nur etwa $10 \%$, aber im Vergleich zu den anderen Varianten am häufigsten genannt wurde die Eigenschaft des Weidelgrases als traditionelle Feldfrucht. 


\subsection{Gesamtindex der relativen Anbauwürdigkeit - IrA}

\subsubsection{Bewertung der Varianten mit dem Gesamtindex IrA}

An beiden Standorten erwies sich das Deutsche Weidelgras als anbauwürdigste Variante ( $\mathrm{Ab}$ bildung 23, Abbildung 24) mit IrA-Werten von 6,6 am Standort Reinshof und 8,9 am Standort Schoningen. Das als Dauerkultur angebaute Deutsche Weidelgras erreichte an den beiden Standorten, Reinshof und Schoningen, hohe positive Werte bei den Teilindizes Nmin, Wurzeltrockenmasse und Spurenelemente sowie, wenn auch weniger deutlich ausgeprägt, beim Teilindex Bodenwasser. Am Standort Schoningen zeigte diese Variante zudem bei weiteren Teilindizes ihre Stärken. So hatte das Deutsche Weidelgras hier insbesondere einen hohen Wert beim Teilindex Ökonomische Aspekte. Auch die Teilindizes TM-Ertrag und Methan kamen der Gesamtbewertung zugute. So konnten beim Deutschen Weidelgras am Standort Schoningen nahezu alle Teilindizes zu einem hohen positiven Wert für den Index IrA beitragen - der bei dieser Variante damit sogar mehr als doppelt so hoch ausfiel im Vergleich zur zweitanbauwürdigsten Variante. Die hohe Anbauwürdigkeit stützt sich damit auf ein breites Spektrum an Kenngrößen, die für den Energiepflanzenanbau relevant sind. Am Standort Reinshof war das Deutsche Weidelgras bis auf die genannten Teilindizes (Nmin, Wurzeltrockenmasse, Spurenelemente) eher unauffällig. Insbesondere bei den Teilindizes, die auf die Bewertung der Leistungsfähigkeit (z.B. TM-Ertrag, Deckungsbeitrag) abzielen, hob sich diese Variante eher nicht vom Mittel aller Varianten ab. Dennoch konnte es durch die starke Überlegenheit bei den genannten drei Teilindizes auch hier den mit Abstand höchsten IrA-Wert erreichen.

Den, nach Mais, zweitgrößten Umfang an der Anbaufläche von Energiepflanzen für die Biogaserzeugung in Deutschland mit etwa $15 \%$ hatten im Jahr 2019 Gras und Grünlandaufwüchse inne (FNR 2020). Hinsichtlich der Wirkung der ökologischen und z.T. auch ökonomischen Vorteile, wie sie in der vorliegenden Untersuchung für das Deutsche Weidelgras ermittelt werden konnten, ist ein hoher Umfang von Grasanbauflächen günstig zu bewerten. Techow et al. (2011) zufolge wird das Deutsches Weidelgras aber insbesondere auf Grenzstandorten für den Maisanbau, z.B. auf norddeutschen Marschstandorten, bevorzugt angebaut, da hier das Deutsche Weidelgras durch höhere Erträge und eine höhere Ertragsstabilität dem Mais überlegen ist. Eine Überlegenheit des Deutschen Weidelgrases gegenüber anderen Feldfrüchten - auch gegenüber Mais - zeigt sich anhand der hohen IrA-Werte aber auch für weitaus günstiger einzustufende Standorte in Südniedersachsen. Dabei ist aber im Blick zu behalten, dass Weidelgrasarten, auch das Deutsche Weidelgras, weltweit zunehmend als Unkraut in Erscheinung treten und die Ursachen dafür noch nicht ganz verstanden sind (Petersen 2020).

Deutlich höher als die Anbaufläche von Gras zur Biogaserzeugung ist die von Silomais zur Biogaserzeugung mit etwa $63 \%$ an der gesamten Energiepflanzenanbaufläche im Jahr 2019 (FNR 2020). Ob es sich dabei um Mais als Hauptfrucht nach Schwarzbrache, Mais als Hauptfrucht nach einer abfrierenden Zwischenfrucht oder um Mais als Zweitfrucht im Zweikulturnutzungssystem handelt, kann der Statistik nicht entnommen werden. Für eine Aussage zur Bewertung des Anbauumfangs ist diese Information aber unerlässlich. Denn, wie die Werte für den Index IrA zeigen, hat der Mais als Hauptfrucht nur eine geringe Anbauwürdigkeit. Die Schwächen bei den ökologischen Kenngrößen können nicht durch die Stärken bei der Leis- 
tungsfähigkeit kompensiert werden. Ganz anders verhält es sich bei den Varianten des Zweikulturnutzungssystems. Durch die Erstfrüchte in diesem Anbausystem verbessert sich zumindest am Standort Reinshof durch das Zusammenspiel ökologisch aber auch ökonomisch bedeutsamer Kenngrößen die Anbauwürdigkeit gegenüber Mais als Hauptfrucht deutlich. An den Standort Schoningen sind die Varianten des Zweikulturnutzungssystems jedoch offenbar weniger gut angepasst. Einen deutlich positiven IrA-Wert hat hier allein das Gemenge aus Winterackerbohnen und Wintertriticale gefolgt von Mais als Zweitfrucht, wobei dieser Wert verhältnismäßig stark vom Teilindex Wurzeltrockenmasse geprägt wird.

Wenn auch mit einem nicht sehr hohen IrA-Wert bewertet (1,6 gegenüber 8,9 beim Deutschen Weidelgras), sind zumindest am Standort Schoningen auch die Sommerackerbohnen hinsichtlich der Anbauwürdigkeit positiv zu bewerten. Bei Input von Sommerackerbohnen in den Biogasfermenter kann mit dem größten Beitrag zur Spurenelementversorgung aller hier untersuchten Feldfrüchte gerechnet werden, wie am hohen positiven Teilindex Spurenelemente ersichtlich. Auch über den vergleichsweise hohen Eintrag an Wurzeltrockenmasse in den Boden, zeichnet sich diese Variante aus. Auf der anderen Seite stehen diesen Stärken aber auch große Schwächen gegenüber - insbesondere bei den Kenngrößen, die die Leistungsfähigkeit bewerten. Am Standort Reinshof haben diese Schwächen durch die diskutierten sehr hohen Trockenmasse- und Methanflächenerträge der ertraglich hochleistenden Varianten ein noch größeres Gewicht als am Standort Schoningen, sodass am Standort Reinshof der IrA-Wert negativ ist. Möglichkeiten, die zur Behebung der Schwächen bei den Sommerackerbohnen beitragen können, wurden bereits angesprochen (z.B. die gemeinsame Silierung mit Stroh um die geringen TS-Gehalte zu kompensieren oder Raps als Folgefrucht zur Verbesserung der ökonomischen Bewertung). Stinner et al. (2018) sehen durch den Energiepflanzenanbau zur Biogaserzeugung, bei dem auch Leguminosen in Reinsaat und Gemenge eingesetzt werden können, eine neue Chance für den Leguminosenanbau. So gehen die Autoren davon aus, dass bis zu 20 \% Leguminosen und dabei insbesondere Futterleguminosen auf der gesamten Energiepflanzenanbaufläche integriert werden können ohne dabei die Nahrungs- und Futtermittelkonkurrenz weiter zu verschärfen. Dies, so Stinner et al. (2018), sei v.a. möglich durch die mit der $\mathrm{N}_{2}$-Fixierung verbundenen Energieeinsparung, wodurch wiederum der Anbau von anders genutzten Energiepflanzen, etwa zur Herstellung von Biodiesel und Bioethanol, reduziert könnte. Ähnlich dem Nährstoffkreislauf im ökologischen Landbau würden dabei die Leguminosen den energieaufwendigen Prozess bei der Herstellung von synthetischen Düngemitteln ersetzen, gleichzeitig bei der Vergärung der Biomasse z.B. Strom und Wärme erzeugen und mit dem Gärrest die N-Versorgung von Marktfrüchten sicherstellen. Stinner et al. (2018) fordern daher auch, bei der Diskussion um die Bewertung von Feldfrüchten die $\mathrm{N}_{2}$ Fixierleistung bei Leguminosen und den Energieverbrauch beim Anbau von Nicht-Leguminosen einzubeziehen. Dies, so wurde bereits angesprochen, könnte mithilfe eines Teilindex Ressourcennutzung abgebildet werden. Durch einen höheren Anteil an Leguminosen als Biogassubstrat könnte möglicherweise auch ein geschlossener Kreislauf hinsichtlich der Spurenelementversorgung der Mikroorganismen im Biogasprozess ohne die Zugabe von Spurenelementpräparaten erreicht werden.

Die Attraktivität des Leguminosenanbaus zur Biogaserzeugung kann auch über den von Dieckmann et al. (2018) vorgeschlagenen Aspekt gesteigert werden: Diesen Autoren zufolge kann der Biomasseaufwuchs nicht nur zur Biogaserzeugung genutzt werden, sondern zusätz- 
lich auch stofflich. Aufgrund des hohen Proteingehalts wäre bei Leguminosen vorstellbar, zunächst die Proteinfraktion aus der Biomasse abzuziehen und daran anschließend den überbleibenden Rest dem Biogasprozess zuzuführen. Das Protein könnte dann in der Nahrungsoder Futtermittelindustrie als hochwertiger Rohstoff eingesetzt werden. Bei einer Modellrechnung mit Kleegras kamen Dieckmann et al. (2018) zu einer deutlichen Steigerung der Wertschöpfung durch die zusätzliche stoffliche Nutzung des Kleegrases.

An beiden Standorten, Reinshof und Schoningen, zeigt auch die Durchwachsene Silphie einen positiven IrA-Wert. Bis auf den etwas gewichtigeren positiven Teilindex Nmin am Standort Reinshof, ist die Durchwachsenen Silphie aber eher unauffällig. Das bedeutet, dass diese Variante bei nahezu allen untersuchten Kenngrößen etwa der mittleren Ausprägung aller untersuchten Varianten entspricht, sie also keine besonders großen Schwächen, aber auch nur wenig besonders große Stärken hat. Dennoch ist auf der Grundlage des vorliegenden Bewertungsschemas ihre Anbauwürdigkeit dem Mais als Hauptfrucht nach Schwarzbrache gegenüber gleichgestellt (Schoningen) oder sogar deutlich günstiger (Reinshof). Eine Verbesserung bei der ökonomischen Leistungsfähigkeit wurde bereits hinsichtlich der Kostensenkung durch Etablierung als Untersaat in Mais diskutiert. Zum agrarökologischen Potenzial der Durchwachsenen Silphie zählen u.a. die ausbleibende Bodenbearbeitung, der Blühaspekt und die Förderung von Bestäuberinsekten (Schorpp et al. 2016). Diese Vorteile könnten stärker berücksichtigt werden. Die Durchwachsenen Silphie würde so weiter an Vorzüglichkeit gewinnen. Eine Verzehnfachung der Anbaufläche innerhalb von fünf Jahren in Deutschland von 300 ha im Jahr 2013 auf 3000 ha im Jahr 2018 (FNR 2020) weisen in diese Richtung.

Die ein- und mehrjährige Blühmischung sowie der Amarant bilden das Schlusslicht in der Bewertung mit deutlich negativen Werten im Index IrA. Die Schwächen überwiegen bei diesen Varianten ganz deutlich gegenüber den Stärken. IrA und die Teilindizes lassen die Schwächen aber klar erkennen und liefern somit wertvolle Hinweise, bei welchen Merkmalen die Anbauwürdigkeit dieser Varianten in Zukunft verbessert werden kann.

Hinsichtlich der Spurenelementlieferung ist die Durchwachsene Silphie ebenfalls unauffällig. Dies trifft, anders als aufgrund der Ergebnisse von Sauer et al. (2014) erwartet, auch auf die ein- und mehrjährige Blühmischung sowie den Amarant zu. Umso erstaunlicher sind die Ergebnisse des Verbundprojekts, bei dem eine Substitution der Spurenelementpräparate über die Zumischung von $30 \%$ alternativen Energiepflanzen zu $70 \%$ Mais getestet wurden, wobei hier Winterackerbohnen, Amarant, Deutsches Weidelgras und die Durchwachsene Silphie eingesetzt wurden. Auf die Zugabe von Spurenelementpräparaten konnte dabei zwar nicht vollständig verzichtet werden, aber $25 \%$ der üblichen Menge reichten bei allen vier Varianten bereits aus, um einen stabilen Biogasprozess bei vergleichsweise hoher Raumbelastung zu ermöglichen. Bei der Monovergärung von Mais hingegen reichte ein Anteil von $25 \%$ der üblichen Spurenelementmenge nicht aus, den Biogasprozess stabil zu erhalten (Sauer et al. 2018). Da sich die Spurenelementmengen beim Einsatz von Ackerbohnen, Amarant, Deutschem Weidelgras und der Durchwachsenen Silphie, wie gezeigt werden konnte, unterscheiden, legt dies die Erwartung nahe, dass beim Einsatz von Ackerbohnen für einen optimalen Biogasprozess sogar auf noch mehr Spurenelementpräparat verzichtet werden könnte als es bisher der Fall war. 


\subsubsection{Bewertung des Index IrA}

Die Teilindizes im Index IrA sind so konstruiert, dass bei Kenngrößen, bei denen hohe Werte positiv bewertet werden, der Teilindex eine Ausprägung von -1 bis $+\infty$ haben kann. Dies ist z.B. beim Teilindex Trockenmasseertrag der Fall. Bei Kenngrößen, bei denen kleine Werte günstiger eingestuft werden als große, ergibt die Indexberechnung andere Randbedingungen. Hier können die Teilindexwerte eine Ausprägung von $-\infty$ bis +1 haben. Dies ist beim Teilindex Nmin der Fall, bei dem geringe Nmin-Mengen zu den relevanten Zeitpunkten günstiger eingeschätzt werden als höhere. Wie bereits Menke (2011) sowie Menke \& Rauber (2015) anmerkten, führt dies dazu, dass besonders hohe Werte bei den Teilindizes, die einen Wert größer +1 annehmen können, IrA in die positive Richtung beeinflussen. Zum Beispiel wäre dies der Fall bei besonders hohen Trockenmasseerträgen einer Variante. Durch hohe Nmin-Mengen hingegen, also dem Teilindex, dessen Wert kleiner -1 sein kann, wird IrA möglicherweise in die negative Richtung beeinflusst. Daher schlägt Menke (2011) vor, die Berechnung der Teilindizes so zu gestalten, dass sie im positiven wie auch negativen Bereich die gleichen Randbedingungen aufweisen. Dies ist der Fall bei dem hier neu eingeführten Teilindex Ökonomische Aspekte. Bei diesem Teilindex kann die Ausprägung von $-\infty$ bis $+\infty$ reichen. Zustande kommt dies, da neben einem positiven Betriebsergebnis auch ein negatives möglich ist, die in die Teilindexberechnung eingehenden Werte also sowohl positiv wie auch negativ sein können. Neben dem Teilindex ökonomische Aspekte wäre eine solche Teilindexberechnung möglicherweise auch beim Teilindex Nmin denkbar. Dies wäre der Fall, wenn bei der Berechnung anstatt von den Nmin-Mengen selbst, von der Menge an abgesenktem Nmin, z.B. im Winter gegenüber der Schwarzbrache, ausgegangen würde. Hierbei wären größere Werte günstiger einzuschätzen als geringe. Ein so konstruierter Teilindex Nmin hätte, wie auch der Teilindex Ökonomische Aspekte, eine Ausprägung von $-\infty$ bis $+\infty$, da es sowohl zu einer Absenkung des Nmin gegenüber der Schwarzbrache kommen kann, z.B. beim Anbau einer Getreide-Erstfrucht im Zweikulturnutzungssystem, als auch zu einer Zunahme z.B. beim Anbau von Winterackerbohnen als Erstfrucht im Zweikulturnutzungssystem. Bei Kenngrößen, wie z.B. dem Trockenmasseertrag hingegen, bei denen es keine negativen Ausprägungen geben kann, scheint eine solche Berechnungsweise nicht möglich zu sein.

Der Index IrA und die Teilindizes müssen, um eine Schwachstellenanalyse zu ermöglichen, weitgehend einfach, transparent und nachvollziehbar sein. Das ist möglich, wenn in einen Teilindex auch nur genau ein Parameter eingeht. Das ist bei den meisten, in der vorliegenden Untersuchung in IrA einbezogenen Teilindizes der Fall. So geht z.B. der Teilindex TM-Ertrag aus den Trockenmasseerträgen der Feldfrüchte hervor oder der Teilindex Ökonomische Aspekte aus den Deckungsbeiträgen. Anders ist dies jedoch bei Teilindizes, bei denen mehr als ein Parameter oder Daten von mehr als einem Zeitpunkt eingehen. Dann kann es schwierig sein, die Information richtig zu interpretieren, um Handlungsempfehlungen abzuleiten. In der vorliegenden Untersuchung trifft dies auf den Teilindex Nmin zu, in den sowohl die Bewertung der Nmin-Mengen im Winter als auch die nach der Ernte der Feldfrüchte eingehen. So ist z.B. beim Zweikulturnutzungssystem aus Winterackerbohnen und nachfolgendem Mais offensichtlich, dass es hier eine Schwäche beim Nmin gibt (Abbildung 23, Abbildung 24). Unklar bleibt jedoch, an welcher Stellschraube gedreht werden müsste. Denn, wie bereits diskutiert wurde, sind neben den vergleichsweise hohen Nmin-Mengen im Winter bei Winterackerbohnen insbesondere die hohen Nmin-Mengen nach der Ernte der Zweitfrucht Mais ausschlaggebend für 
den großen negativen Teilindexwert. Ähnlich verhält es sich z.B. auch beim Teilindex Spurenelemente. In diesen Teilindex gehen sowohl die Cobalt- als auch die Nickelabfuhren ein. Bei einem hohen Wert für den Teilindex Spurenelemente, wie inn z.B. die Sommerackerbohnen zeigen, kann also keine Aussage darüber getroffen werden, ob, wie gewünscht, beide Elemente in hohen Mengen vorhanden sind oder nur Cobalt oder nur Nickel. Die Überlegung sollte also eher dahingehen, die Teilindizes so zu konstruieren, dass möglichst hohe Transparenz gegeben ist. Der einfachste Weg wäre, die jeweils verschiedenen Kenngrößen, in eigenen Teilindizes darzustellen.

Durch die Vorgehensweise, die Werte der Teilindizes im Gesamtindex IrA aufzuaddieren, können negative Werte durch positive kompensiert werden. So kann es sein, dass trotz vielfältiger Schwächen der Wert für den Gesamtindex IrA positiv ist. Dies kann die Gefahr bergen, Ziele zu verfehlen. Wenn das Ziel ist, eine Feldfrucht v.a. dann positiv zu bewerten, wenn sie sowohl bei ökonomischen als auch ökologischen und sozialen Kenngrößen günstige Ausprägungen hat, dann sind mögliche Kompensationen einer Kenngröße durch eine andere widersprüchlich. Denn durch die Kompensation könnte ein gesamter Schwerpunkt, z.B. die ökologischen Kenngrößen, negativ sein, der Gesamtindex IrA aber dennoch positiv. So ergab sich z.B. am Standort Schoningen ein knapp positiver IrA-Wert für den Mais, obwohl dieser nur bei den KenngröBen zur Leistungsfähigkeit (TM-Ertrag, Methan-Ertrag, Deckungsbeitrag) sowie dem TS-Gehalt positive Teilindexwerte erzielte. Insbesondere bei den ökologisch ausgerichteten Teilindizes wurde diese Variante also entsprechend ungünstig bewertet. Andersherum erzielten aber die Sommerackerbohnen am Standort Schoningen einen positiven IrA-Wert, obwohl bei dieser Variante sämtliche Teilindizes negativ waren, die die Leistungsfähigkeit bewerten. Da der Index IrA mit seinen Teilindizes eine Schwachstellenanalyse ermöglichen soll, auf dessen Grundlage Anbauentscheidungen in der Praxis getroffen werden könnten, ist der IrA-Wert allein ohnehin nur wenig aussagekräftig. Es ist vielmehr zwingend notwendig, auch die Ausprägungen der Teilindizes zu berücksichtigen. Zur Bildung einer Rangfolge zwischen Varianten ist der Index IrA aber dennoch geeignet. Denn wie die Ergebnisse der vorliegenden Untersuchung zeigen, haben die Varianten, die besonders gut bewertet werden, also einen deutlich positiven IrA-Wert aufweisen, auch überwiegend günstige Ausprägungen bei den Teilindizes. Varianten, die eher schlecht bewertet werden, also einen deutlich negativen IrA-Wert besitzen, sind durch überwiegend ungünstige Ausprägungen gekennzeichnet. Varianten, bei denen sich der IrA um den Wert Null bewegt, sind eben die Varianten, bei denen sowohl ausgeprägte Stärken als auch ausgeprägte Schwächen zu finden sind bzw. solche, bei denen weder Stärken noch Schwächen vorliegen. Auch bei der Nachhaltigkeitsbewertung landwirtschaftlicher Betriebe mittels des Bewertungsverfahrens „Kriteriensystem nachhaltige Landwirtschaft“ (KSNL) wird das Analyseergebnis für jeden einzelnen der dabei berücksichtigten großen Vielzahl an Kriterien optisch dargestellt (Breitschuh et al. 2008). Es kann auf einen Blick erkannt werden, wo Stärken liegen und wo Schwächen. Das ist mit den Teilindizes im Index IrA hinsichtlich der Bewertung verschiedener Feldfrüchte und der möglicherweise darauf beruhenden Entscheidungsfindung zum Anbau ebenfalls möglich.

Einen ähnlich dem Index IrA aufgebauten Index nutzten auch Fuchs \& Stockfisch (2009) bei der Bewertung der Effizienz des Faktoreinsatzes im Zuckerrübenanbau auf verschiedenen Schlägen. Dabei leiteten die Autoren zunächst verschiedene Effizienzkriterien aus den Produktionsprozessen (Bodenbearbeitung, Düngung, Pflanzenschutz, Ernte) im Zuckerrübenan- 
bau ab, indem die Prozesse selbst ins Verhältnis zum Bereinigten Zuckerertrag gesetzt wurden. Die so ermittelten Effizienzgrößen drücken z.B. aus, wie viel Energie bei der Bodenbearbeitung im Verhältnis zum Ertrag verbraucht wurde. Zur Teilindexberechnung wurde dann, ähnlich der Berechnung des Index IrA, das Effizienzkriterium jedes Schlages ins Verhältnis zum Mittel aller Schläge gesetzt. Schließlich addierten die Autoren die Werte der Teilindizes für jeden Schlag zur Effizienz-Indexzahl auf. Anders als im Index IrA wurden bei Fuchs \& Stockfisch (2009) mit den Effizienzgrößen indirekte Werte zur Berechnung der Teilindizes verwendet. Neben der Leistung der Zuckerrüben wird dabei auch das Management auf den verschiedenen Schlägen bewertet. Ein solches Vorgehen hilft möglicherweise, die Vergleichbarkeit zwischen Standorten zu erreichen.

Eine Schwachstellenanalyse und daraus abgeleitete Handlungsoptionen sind auch das Ziel des KSNL-Modells. Anders als in der vorliegenden Untersuchung geht es dabei aber um die Bewertung der Nachhaltigkeit landwirtschaftlicher Betriebe auf den Ebenen Wirtschafts-, Umwelt- und Sozialverträglichkeit. Jedes Kriterium wird dabei anhand eines vorher definierten Toleranzbereichs bewertet, der angibt, ob ein Zustand optimal (Boniturnote 1) bis hin zu hoch risikoreich (Boniturnote 11) ist (Breitschuh et al. 2008). Ein so ausgerichtetes Vorgehen setzt allerdings voraus, dass die Toleranzbereiche für die Bewertung bekannt und die Schwellen, ab wann eine bestimmte Note vergeben wird, genau abgegrenzt sein müssen. In der vorliegenden Untersuchung hingegen ergaben sich die Schwellen, welcher Zustand positiv und welcher negativ ist, aus den Feldfrüchten selbst durch den Vergleich mit den anderen Feldfrüchten - den Feldfrüchten wurde also selbst eine Stimme gegeben. Vermutlich ist dies der wesentliche Unterschied zu vielen anderen Bewertungsmodellen.

Das Vorgehen, den Feldfrüchten selbst eine Stimme zu geben, könnte allerdings dann an seine Grenzen stoßen, wenn alle einbezogenen Varianten ungünstig sind. Hier kann es aber helfen, wie es in der vorliegenden Untersuchung vorgenommen wurde, ein großes Spektrum an verschiedenartigen Varianten in den Vergleich einzubeziehen. Anhand der Analyse der Teilindizes des Index IrA können Teilindizes identifiziert werden, die die Grenzen des Ansatzes aufzeigen oder sogar zu keinem zusätzlichen Erkenntnisgewinn führen: So ist der Teilindex TS-Gehalt beim Zweikulturnutzungssystem so konstruiert, dass neben der Erst- auch die Zweitfrucht einbezogen wurde. Damit wurde ein gedanklicher Mittelwert der Biomassen gebildet, obwohl in der Praxis mehrere Monate zwischen der Ernte der jeweiligen Komponente liegen. Damit werden geringe TS-Gehalte der Erstfrucht möglicherweise überdeckt.

Wie die Ergebnisse der vorliegenden Untersuchung zeigen und bereits diskutiert wurde, sind die Werte für den Teilindex Bodenwassergehalte der Feldfrüchte eher geprägt von den Ernteterminen und den im Verlauf der Vegetationszeit vorhandenen Niederschlagsmengen als vom Effekt der Feldfrüchte selbst. Die Bodenwassergehalte sind eher eine Momentaufnahme. Der nur geringe Anteil am Gesamtindex IrA von nur $3 \%$ am Standort Reinshof spiegelt dies wider. Die Beanspruchung des Bodenwasserhaushalts einzuschätzen, insbesondere bei Zweikulturnutzungssystemen, behält aber ihre Wichtigkeit. Alternativ zu den Bodenwassergehalten an den verschiedenen Ernteterminen könnte vielleicht eher der Wasserverbrauch der Feldfrüchte berechnet (Abschnitt 4.1.6) und in den Teilindex Bodenwasser einbezogen werden.

Wie bereits angesprochen, zeigt auch die Einbeziehung der beiden Teilindizes Trockenmasseertrag und Methanflächenertrag in den Index IrA Schwachpunkte. Beide Kenngrößen sind 
wichtig für die landwirtschaftliche Praxis und bestimmen letztlich die Konkurrenzfähigkeit einer Feldfrucht. Da aber der Methanflächenertrag wesentlich stärker vom Trockenmasseertrag abhängt als vom spezifischen Methanertrag, geht der Trockenmasseertrag auch mehrfach in den Index IrA ein. Das damit einhergehende hohe Gewicht des Trockenmasseertrags birgt die Gefahr, dass Varianten, die sich im Trockenmasseertrag deutlich vom Mittel aller Varianten unterscheiden, je nach Ausrichtung besser oder schlechter gestellt werden. Eine Möglichkeit diesem Problem Abhilfe zu schaffen wäre, anstatt des Methanflächenertrags, den spezifischen Methanertrag in den Teilindex Methan einfließen zu lassen. Andererseits gibt es Hinweise auf den geringen Effekt der spezifischen Methanausbeuten der Einzelkomponenten auf den Methanertrag in der Fermentermischung (Abschnitt 4.1.3), sodass auch auf den Teilindex Methan verzichtet werden könnte.

Demgegenüber wäre aber eine Erweiterung des Index IrA um weitere Teilindizes denkbar:

Im Rahmen eines Teilindex Bodenbedeckung könnte der Grad und die Geschwindigkeit der Bodenbedeckung und der Aufbau eines Blätterdachs erfasst werden. Damit könnten die Feldfrüchte letztlich in ihrer Fähigkeit, Erosion zu vermeiden und Unkraut zu unterdrücken, bewertet werden.

Ein Teilindex Behandlungsstärke könnte die Intensität des Einsatzes von Pflanzenschutzmitteln beim Anbau von Energiepflanzen für die Biogaserzeugung abbilden.

Mit einem Teilindex Biodiversität könnte die agrarökologische Leistung der Feldfrüchte, wie bereits im Zusammenhang mit der Durchwachsenen Silphie angesprochen, abgebildet werden. Zu beachten wäre dabei allerdings, dass Untersuchungen des Insektenvorkommens in Parzellenversuchen, wie dem vorliegenden, nur schwer möglich sind. Die Parzellen sind wahrscheinlich zu klein, um z.B. genau festzustellen, von welcher Variante bestimmte Insekten angelockt wurden.

In einem Teilindex Ressourcennutzung könnten z.B. der bereits angesprochenen N-Düngemittelbedarf der Varianten bzw. die N-Düngemitteleinsparungen im Zusammenhang mit dem Anbau von Leguminosen einfließen.

An einem Teilindex Treibhausgas wird derzeit gearbeitet. Es soll herausgefunden werden, inwieweit ein Potenzial besteht, beim Anbau von Winterzwischenfrüchten bzw. Erstfrüchten im Zweikulturnutzungssystem und Dauerkulturen Treibhausgase einzusparen. Analysiert werden dabei z.B. die Minderung der indirekten Lachgasemission durch Reduzierung der Nitratauswaschung oder die Speicherung von Humus durch zusätzliche Wurzelbiomasse bei Zwischenfrüchten bzw. ausbleibende Bodenbearbeitung bei Dauerkulturen (Hey 2020). 


\section{Schlussfolgerungen}

Als besonders anbauwürdige Energiepflanzen für die Biogaserzeugung stellten sich das Deutsche Weidelgras an den beiden Versuchsstandorten, Reinshof und Schoningen, insbesondere am Standort Reinshof die Varianten des Zweikulturnutzungssystems sowie am Standort Schoningen die Sommerackerbohnen heraus. Auch die Durchwachsene Silphie schnitt an beiden Standorten positiv ab. Durch den Kontext des Verbundprojekts, einen Fokus auf Feldfrüchte zu legen, die aufgrund ihrer Spurenelementakkumulation zur Substitution von Spurenelementpräparaten im Biogasprozess geeignet sind, könnte das Artenspektrum innerhalb des Energiepflanzenanbaus deutlich erweitert werden. Damit könnte sowohl dem hohen Einsatz von Spurenelementpräparaten als auch der geringen Biodiversität im Energiepflanzenbau entgegengewirkt werden. Die Teilindizes im Index IrA zeigen, bei welchen Merkmalen die Anbauwürdigkeit dieser Varianten als Energiepflanzen in Zukunft verbessert werden kann. Beim Zweikulturnutzungssystem aus Winterackerbohnen und Mais z.B. betrifft dies die Absenkung der hohen Nmin-Mengen im Boden im Winter und nach der Ernte der Zweitfrucht, bei den Sommerackerbohnen insbesondere die Verbesserung der Kenngrößen, die die Leistungsfähigkeit bestimmen (TM-Ertrag, Deckungsbeitrag). 
In hauptsächlich mit Mais beschickten Biogasanlagen kommt es häufig zu einem Mangel an Spurenelementen, v.a. Cobalt und Nickel. Der Grund dafür ist, dass Mais nur ein geringes Aufnahmevermögen für diese prozessrelevanten Spurenelemente aufweist. Um diesem Mangel zu begegnen, werden in der Praxis künstlich hergestellte Spurenelementpräparate eingesetzt. Dieser Einsatz kann negative Auswirkungen sowohl auf den Anwender als auch die Umwelt haben, da es sich dabei um Schwermetalle handelt. Alternative Energiepflanzen akkumulieren im Vergleich zu Mais erheblich mehr Spurenelemente. Die Hypothese ist, dass durch die Zumischung von Pflanzen, die Spurenelemente akkumulieren, eine ausreichende Versorgung für die Vergärung gewährleistet ist und auf die Zugabe von Spurenelementpräparaten verzichtet werden kann. Das Ziel dieser Untersuchung war es, spurenelementakkumulierende Pflanzenarten auf ihre Eignung für den Anbau als Energiepflanzen für die Biogaserzeugung zu untersuchen und mithilfe des Index der relativen Anbauwürdigkeit (IrA, Menke 2011) zu bewerten.

In zweijährigen Feldversuchen (2014/15 und 2015/16) wurden an zwei Standorten in Südniedersachsen (Göttingen Reinshof, Auenlehm und Schoningen bei Uslar, sandiger Schluff) verschiedene Feldfrüchte angebaut und untersucht: Das waren zum einen die Zweikulturnutzungssysteme aus Wickroggen, Winterackerbohnen, Wintertriticale und ein Gemenge aus Winterackerbohnen und Wintertriticale (Gemenge WABNT) als Erstfrüchte, jeweils gefolgt von Mais als Zweitfrucht, zum anderen die Sommerhauptfrüchte Sommerackerbohnen, eine einjährige Blühmischung, Amarant, Mais und ein Gemenge aus Amarant und Mais. Außerdem gab es die mehrjährigen Arten Deutsches Weidelgras, eine mehrjährige Blühmischung und Durchwachsene Silphie, die als Dauerkulturen angelegt wurden. Aus Vorversuchen war bekannt, dass besonders Winter- und Sommerackerbohnen, die im Wickroggen enthaltene Winterwicke, einzelne, in den Blühmischungen enthaltene Arten, der Amarant und das Weidelgras reich an Cobalt und Nickel sind. Es fand ein praxisüblicher Einsatz von Dünge- und Pflanzenschutzmitteln statt. Die Ernte erfolgte praxisüblich.

Zahlreiche Parameter wurden erhoben: Bei der Ernte zur Siloreife wurde anhand der Biomasseproben der Trockenmasseertrag und der Trockensubstanzgehalt bestimmt. An den getrockneten Biomasseproben wurden im praktischen Gärversuch (Hohenheimer Biogasertragstest) die spezifischen Methanerträge der Feldfrüchte ermittelt sowie nach Säuretotalaufschluss mittels Emissions- sowie Massenspektrometrie die Spurenelementgehalte analysiert - insgesamt 47, von denen Cobalt und Nickel hier betrachtet werden. Zudem wurde der mineralische Stickstoff im Boden in drei Tiefenstufen bis $90 \mathrm{~cm}$ sowohl im Winter (Erstfrüchte, Dauerkulturen, Schwarzbrache) als auch nach der Ernte der Feldfüchte gemessen. Der Bodenwassergehalt wurde in einer Tiefe bis $80 \mathrm{~cm}$ gravimetrisch zu Vegetationsbeginn sowie nach der Ernte der Feldfrüchte bestimmt. Um die Mengen an pflanzenverfügbarem Wasser zu ermitteln, wurde für beide Standorte eine pF-Kurve angefertigt. Außerdem wurden zur Ernte der Feldfrüchte mithilfe von Rammkernsonden Bodenproben zur Ermittlung der Wurzelmasse bis zu einer Tiefe von $60 \mathrm{~cm}$ am Standort Reinshof und $30 \mathrm{~cm}$ am Standort Schoningen aus dem Boden entnommen. Nach Auswaschung der Proben wurde die Wurzeltrockenmasse bestimmt. Weiterhin wurde eine Online-Umfrage mithilfe des Tools "LimeSurvey“ in der Bevölkerung durchgeführt, um die gesellschaftliche Akzeptanz der untersuchten Energiepflanzenvarianten zu erheben. Insgesamt gingen 575 Fragebogen ein, von denen 446 vollständig waren 
und damit auswertbar. Schließlich wurde auf Grundlage der im Versuch gewonnenen Daten eine Teilkostenrechnung erstellt und der Deckungsbeitrag für jede Variante ermittelt.

Zur Bewertung der Varianten wurde der Index der relativen Anbauwürdigkeit (IrA) weiterentwickelt. Der Index IrA setzt sich nun aus neun Teilindizes zusammen:

- Spurenelementgehalt (Cobalt und Nickel)

- Nmin-Menge im Boden über Winter und nach der Ernte

- Trockenmasseertrag (TM-Ertrag)

- Methanflächenertrag

- Trockensubstanzgehalt (TS-Gehalt)

- Pflanzenverfügbares Wasser im Boden nach der Ernte

- Wurzeltrockenmasse

- Soziale Aspekte (Akzeptanz der untersuchten Feldfrüchte in der Bevölkerung)

- Ökonomische Aspekte.

Der Wert eines Teilindex für eine Variante ergibt sich daraus, inwieweit die jeweilige Kenngröße, z.B. der Trockenmasseertrag einer Variante, vom Mittel aller untersuchten Varianten abweicht. Günstige Ausprägungen einer Kenngröße, z.B. ein vergleichsweise hoher Trockenmasseertrag oder eine geringe Nmin-Menge im Boden im Winter, führen zu hohen Werten der Teilindizes, ungünstige Ausprägungen entsprechend zu niedrigen. Die Summe der Teilindizes ergibt den Gesamtindex IrA. Je größer der Gesamtindex IrA ist, umso besser wird eine Variante eingestuft. Bei den Varianten des Zweikulturnutzungssystems gehen sowohl die Erst- als auch die Zweitfrüchte in die Indexberechnungen ein.

Am Standort Reinshof bewegten sich die Trockenmasseerträge zwischen 92 und 302 dt TM ha $^{-1}$. Die höchsten Trockenmasseerträge wurden hier von den Varianten des Zweikulturnutzungssystems erreicht, gefolgt von Mais als Hauptfrucht, dem Deutschen Weidelgras und der Durchwachsenen Silphie. Eine Gruppe mit deutlich geringeren Trockenmasseerträgen bildeten der Amarant, die ein- und mehrjährige Blühmischung sowie die Sommerackerbohnen. Am Standort Schoningen war das Ertragsniveau geringer als am Standort Reinshof und bewegte sich zwischen 41 und $230 \mathrm{dt} \mathrm{TM} \mathrm{ha}{ }^{-1}$. Auch am Standort Schoningen ließen sich Gruppen mit unterschiedlichen Trockenmasseerträgen ausmachen. Hier allerdings zogen der Mais als Hauptfrucht sowie im Gemenge mit Amarant, das Deutsche Weidelgras und die Durchwachsene Silphie gleich mit den Varianten des Zweikulturnutzungssystems. Der Amarant, die einund mehrjährige Blühmischung sowie die Sommerackerbohnen blieben auch am Standort Schoningen weit hinter diesen zurück.

Die für eine verlustfreie Silierung angestrebten Trockensubstanzgehalte zur Biomasseernte von 28-35 \% konnten an beiden Standorten insbesondere von Mais und dem Gemenge aus Amarant und Mais erreicht werden. Am Standort Reinshof traf dies auch auf die mehrjährige Blühmischung sowie am Standort Schoningen auf die Durchwachsene Silphie zu. Nah an diesen Bereich heran kamen teilweise auch die getreidebetonten Erstfrüchte des Zweikulturnutzungssystems. Alle übrigen Varianten blieben z.T. deutlich dahinter zurück. Die Winterackerbohnen wiesen mit etwa $14 \%$ die geringsten TS-Gehalte auf.

Die Methanflächenerträge bewegten sich am Standort Reinshof zwischen $2500 \mathrm{Nm}^{3} \mathrm{CH}_{4} \mathrm{ha}^{-1}$ bei der mehrjährigen Blühmischung des ersten Versuchsjahres und $9600 \mathrm{Nm}^{3} \mathrm{CH}_{4} \mathrm{ha}^{-1}$ beim Zweikulturnutzungssystem aus Wickroggen gefolgt von Mais. Während die Varianten des Zweikulturnutzungssystems im ersten Versuchsjahr am Standort Reinshof mit dem Mais als 
Hauptfrucht gleichzogen, überboten die Zweikulturnutzungsvarianten diesen im zweiten Versuchsjahr deutlich. Das Deutsche Weidelgras und die Durchwachsene Silphie erzielten mittlere Methanflächenerträge von 5200 bis $6000 \mathrm{Nm}^{3} \mathrm{CH}_{4} \mathrm{ha}^{-1}$. Am Standort Schoningen lagen die Methanflächenerträge, wie auch die Trockenmasseerträge, auf einem insgesamt niedrigeren Niveau als am Standort Reinshof. Der Abstand zwischen den Varianten war am Standort Schoningen mit Werten zwischen $1000 \mathrm{Nm}^{3} \mathrm{CH}_{4}$ ha' ${ }^{-1}$ bei der einjährigen Blühmischung und $7300 \mathrm{Nm}^{3} \mathrm{CH}_{4}$ ha-1 beim Zweikulturnutzungssystem aus Wintertriticale gefolgt von Mais noch deutlich größer als am Standort Reinshof. Die Varianten des Zweikulturnutzungssystems unterschieden sich am Standort Schoningen nicht von Mais als Hauptfrucht. Allerdings konnte hier auch das Deutsche Weidelgras in die Gruppe der ertragsstärksten Varianten eingeordnet werden.

Hinsichtlich der Aufnahme an Cobalt und Nickel hoben sich insbesondere die Sommer- und Winterackerbohnen mit hohen Gehalten ab. So hatten die Sommerackerbohnen einen um mehr als 20-fach höheren Cobaltgehalt als der Mais. Beim Nickel traf dies, wenn auch nicht mit ganz so großem Abstand zu den anderen Varianten, ebenfalls auf das Deutsche Weidelgras zu.

Auch hinsichtlich der im Boden zurückgebliebenen Wurzelmassen unterschieden sich die Varianten an beiden Standorten stark. So bildeten der Amarant, der Mais und auch z.T. die Blühmischungen nur sehr geringe Wurzeltrockenmassen von höchstens $10 \mathrm{dt}$ TM ha ${ }^{-1}$. Demgegenüber waren insbesondere die Sommer- und Winterackerbohnen aber auch das Gemenge aus Winterackerbohnen und Wintertriticale sowie das Deutsche Weidelgras imstande, hohe Wurzeltrockenmasseerträge von bis zu $60 \mathrm{dt} \mathrm{TM} \mathrm{ha-1}^{-1} \mathrm{zu}$ bilden.

Der Anbau von nicht-legumen bzw. getreidedominierenden Erstfrüchten im Zweikulturnutzungssystem führte z.T. zu einer Absenkung der Nmin-Mengen im Boden bis zum Winter gegenüber der Schwarzbrache. Insbesondere am Standort Reinshof unterschieden sich die Winterackerbohnen nicht von der Schwarzbrache. Am Standort Schoningen waren die Effekte der Erstfrüchte auf den Nmin über Winter nicht so eindeutig. An beiden Standorten aber waren die mehrjährigen Arten, Deutsches Weidelgras, die mehrjährige Blühmischung und die Durchwachsene Silphie in der Lage, über Winter die Nmin-Mengen im Boden gering zu halten. Nach der Ernte der Feldfrüchte fiel insbesondere der Mais als Zweitfrucht mit den höchsten residualen N-Mengen auf und dabei v.a. der Mais, der nach Winterackerbohnen folgte.

Besonders große Unterschiede zwischen den Versuchsstandorten Reinshof und Schoningen, aber auch zwischen den Versuchsjahren, wurden bei den Bodenwassergehalten nach der Ernte der Feldfrüchte sichtbar. Im ersten Versuchsjahr entleerten die Erstfrüchte im Zweikulturnutzungssystem den Bodenwasservorrat bis zur Ernte Anfang Juni stark, im zweiten Versuchsjahr unterschieden sich zumindest am Standort Reinshof die Bodenwassergehalte hingegen zur gleichen Zeit nicht wesentlich von der Schwarzbrache. Die Varianten, die im Verlauf der Sommermonate geerntet wurden, hinterließen tendenziell weniger Wasser im Boden als die Varianten, die im Herbst geerntet wurden, schwankten aber auch zwischen den Versuchsjahren. Am Standort Schoningen war im ersten Versuchsjahr zeitweise überhaupt kein pflanzenverfügbares Wasser im Boden zu finden. Zudem unterschieden sich hier die Versuchsjahre stark, sodass es schwierig ist, auszumachen, welche Unterschiede im Bodenwassergehalt durch die Feldfrüchte selbst zustande kamen. 
Die Umfrage in der Bevölkerung ergab ein recht eindeutiges Bild, was die große Akzeptanz von Blühmischungen als Energiepflanzen für die Biogaserzeugung angeht. Der weit überwiegende Teil der Befragten stimmt dem Anbau dieser Feldfrüchte zu. Die Durchwachsene Silphie als ebenfalls blühende Variante, schnitt hingegen weniger gut ab. Die geringste Akzeptanz fand der Amarant, das Gemenge aus Amarant und Mais und der Mais in Reinsaat als Hauptfrucht. Jedoch stimmten auch bei diesen Varianten $40 \%$ der Befragten dem Anbau zur Biogaserzeugung zu.

Aus betriebswirtschaftlicher Perspektive stand am Standort Reinshof der Mais mit Deckungsbeiträgen von bis zu knapp $1400 €$ ha $^{-1}$ sehr gut da. Im zweiten Versuchsjahr konnten hier aber auch die Zweikulturnutzungssysteme und die Durchwachsene Silphie mithalten. Beim Zweikulturnutzungssystem stiegen die Kosten durch den Anbau von zwei Feldfrüchten pro Jahr stärker als die Leistung dadurch stieg. Deutlich geringer als bei den angesprochenen Varianten waren die Deckungsbeiträge von Amarant, der ein- und mehrjährigen Blühmischung sowie den Sommerackerbohnen mit Werten zwischen 400 bis höchstens $700 €$ ha $^{-1}$. Am Standort Schoningen lagen die Deckungsbeiträge auf einem insgesamt niedrigeren Niveau. Bei der einjährigen Blühmischung des zweiten Versuchsjahres konnten die Kosten nicht von den Leistungen gedeckt werden und es ergab sich ein negativer Deckungsbeitrag. An diesem Standort führte der Mais zu einem vergleichsweise guten Betriebsergebnis, insbesondere aber das Deutsche Weidelgras, das mit einem Deckungsbeitrag von $994 €$ ha $^{-1}$ den Mais mit $824 €$ ha $^{-1}$ im zweiten Versuchsjahr sogar übertraf.

Mithilfe der aus den vorgestellten Ergebnissen berechneten Teilindizes konnten die Feldfrüchte relativ zueinander bewertet werden. Die Stärken und Schwächen der einzelnen Feldfrüchte wurden sichtbar. Die Aggregation der Teilindizes zum Gesamtindex IrA ermöglichte schließlich die Erstellung einer Rangliste in Abhängigkeit von der relativen Anbauwürdigkeit. Es zeigte sich, dass an beiden Standorten, aufbauend auf dem neunstufigen Bewertungsschema, das Deutsche Weidelgras die anbauwürdigste Variante war. Am Standort Schoningen vereinte diese Variante nahezu alle Teilindizes im positiven Bereich und setzte sich damit weit von den anderen Varianten ab. Am Standort Reinshof war der Unterschied im IrA zwischen dem Deutschen Weidelgras und den ebenfalls positiv bewerteten Varianten, insbesondere den Varianten des Zweikulturnutzungssystems, nicht ganz so groß. Ebenfalls zu einem positiven IrA-Wert kam an beiden Standorten auch die Durchwachsene Silphie. Damit kann sie neben dem Weidelgras und am Standort Reinshof den Zweikulturnutzungssystemen als Energiepflanze für die Biogaserzeugung empfohlen werden. Vor dem Hintergrund einer möglichst guten Spurenelementversorgung des Biogasfermenters können von den Varianten, die einen positiven IrA-Wert erreichten, also überwiegend günstige Ausprägungen bei den relevanten Kenngrößen zeigten, folgende Varianten empfohlen werden: Das Deutsche Weidelgras an beiden Standorten, die Winterackerbohnen gefolgt von Mais am Standort Reinshof sowie, insbesondere, wenn versucht wird, die Schwächen hinsichtlich der Leistungsfähigkeit auszumerzen, am Standort Schoningen auch die Sommerackerbohnen.

Die Untersuchungen haben gezeigt, dass es möglich ist, mit IrA und den zugehörigen Teilindizes die relative Vorzüglichkeit einer Feldfrucht hinsichtlich der Biogaserzeugung adäquat und transparent abzubilden. Der Index IrA ist so konstruiert, dass er ohne Weiteres erweitert bzw. an besondere Bedürfnisse angepasst werden kann. 


\section{$7 \quad$ Summary}

Biogas plants that are mainly fed with maize, often show a lack of trace elements, especially of cobalt and nickel. The reason for this is the low absorption capacity of maize of these process-relevant trace elements. To counter these deficiencies artificially produced trace element additives are used in practice. This use can have negative effects on the environment as it includes heavy metals. Other potential energy crops accumulate significantly more trace elements compared to maize. The hypothesis is, that the addition of crops that accumulate trace elements, ensures a sufficient supply for fermentation and that the artificial trace element additives can be replaced in this way. The aim of this study was to examine trace element accumulating crop species concerning their suitability for cultivation as energy crops and to evaluate them using the index of relative agronomical suitability (IrA, Menke 2011).

In two-year field trials (2014/15 and 2015/16), different crops were grown and examined at two sites in southern Lower Saxony (Göttingen Reinshof, Alluvial loam and Schoningen near Uslar, sandy silt): Winter faba bean, winter triticale, the intercropping of winter faba bean with winter triticale and the winter cover crop mixture of winter rye and hairy vetch were grown in a double cropping system with maize. The summer main crops faba bean, an annual flower mixture, amaranth, maize and the intercrop of amaranth and maize as well as the perennial crops perennial ryegrass, a perennial flower mixture and cup plant were also investigated. It was evident from precedent tests that particularly winter and summer faba beans, but also the hairy vetch contained in rye, individual species of the flowering mixtures, amaranth and ryegrass are rich in cobalt and nickel.

Numerous parameters were collected: Biomass samples were used to determine the dry matter yield and the dry matter content. The specific methane yield of the crops was determined in the Hohenheim biogas yield test, HBT. 47 trace elements were analysed via total acid digestion and emission and mass spectrometry of which cobalt and nickel are considered here. Mineral nitrogen in the soil (Nmin) was measured at three depths down to $90 \mathrm{~cm}$ both in winter (first crops in the double cropping systems, perennial crops, bare fallow) and after harvest of the crops in the summer or autumn. The soil water content was determined gravimetrically at a depth down to $80 \mathrm{~cm}$ at the onset of vegetation and after harvest of the crops. To determine the amount of plant available water, a pF curve was established for both sites. Soil samples were taken to record the root mass down to a depth of $60 \mathrm{~cm}$ at the Reinshof site and $30 \mathrm{~cm}$ at the Schoningen site. Based on these samples the root dry mass was determined.

An online survey was carried out using the "LimeSurvey" tool to measure the acceptance of the examined energy crops. A total of 575 questionnaires were received, of which 446 were complete and thus evaluable. Based on the data obtained a direct cost calculation was performed and the contribution margin for each variant was determined.

The index of relative agronomical suitability (IrA) was refined to assess the crop variants. The IrA index now consists of nine sub-indices:

- Trace element content (cobalt and nickel)

- Nmin in the soil during winter and after harvest 
- Dry matter yield (DM yield)

- Methane yield per hectare

- Dry matter content (DM content)

- Plant-available water in the soil after harvest

- Root dry matter

- Social aspects (acceptance of the examined crops by the human population)

- Economic aspects

The value of a sub-index for a variant results from the extent to which the respective parameter, e.g. the dry matter yield, deviates from the mean value calculated from all the variants examined. Favourable properties of a parameter, e.g. a comparatively high dry matter yield or a low winter Nmin, lead to high sub-index values. Correspondently, unfavourable properties result in low sub-index values. The sum of the sub-indices results in the overall index IrA. The higher the overall index IrA, the better a variant is evaluated. For the variants of the double cropping system, both the first and the second crop are included in the index calculations.

At site Reinshof the dry matter yields ranged between 9,2 and 30,2 t DM ha ${ }^{-1}$. The highest dry matter yields at site Reinshof were achieved by the variants of the double cropping system, followed by maize as a main crop, ryegrass and cup plant. A group with significantly lower dry matter yields consists of amaranth, the annual and perennial flowering mixture and faba bean. The yield level at the Schoningen site was lower than at the Reinshof site and ranged between 4,1 and 23 t DM ha $^{-1}$. Groups with different dry matter yields could also be identified at the Schoningen site. Here, however, ryegrass and cup plant, maize as a main crop as well as maize in intercropping with amaranth came close to the variants of the double cropping system. Amaranth, the annual and perennial flowering mixture and faba bean were far behind these variants at the Schoningen site, similiar to the Reinshof site.

The target dry matter content for loss-free ensiling (28-35\%) could be achieved at both sites, particularly with maize and the intercrop of maize and amaranth. At the Reinshof site, this also applied to the perennial flowering mixture and to the cup plant at the Schoningen site. In some cases, the grain-based first crops of the double cropping system also came close to this range. All other variants remained clearly behind it. The winter faba bean had the lowest DM content at around $14 \%$.

The methane yields at site Reinshof ranged between $2500 \mathrm{Nm}^{3} \mathrm{CH}_{4}$ ha $^{-1}$ with the perennial flowering mixture of the first experimental year and $9600 \mathrm{Nm}^{3} \mathrm{CH}_{4} \mathrm{ha}^{-1}$ with the double cropping system of rye/hairy vetch followed by maize of the second experimental year. In the first experimental year at site Reinshof the variants of the double cropping system were comparable with maize as a main crop. In the second experimental year, the double cropping system clearly outperformed maize as a main crop. Ryegrass and cup plant achieved average methane yields of 5200 to $6000 \mathrm{Nm}^{3} \mathrm{CH}_{4} \mathrm{ha}^{-1}$. At site Schoningen, methane yield per hectare attained lower levels than at site Reinshof. Comparing the two sites, the distance between the variants at site Schoningen was significantly larger with values between $1000 \mathrm{Nm}^{3} \mathrm{CH}_{4} \mathrm{ha}^{-1}$ for the annual flowering mixture and $7300 \mathrm{Nm}^{3} \mathrm{CH}_{4}$ ha $^{-1}$ for the double cropping system of winter triticale followed by maize. The variants of the double cropping system did not differ from maize as a main crop at site Schoningen. However, the ryegrass could also be classified here as one of the highest yielding variants. 
Regarding the accumulation of cobalt and nickel, the summer and winter faba bean variants stood out with very high contents. The summer faba bean showed a cobalt content that was more than 20 times higher than that of maize. In the case of nickel, this was also true for ryegrass, but with less distance to the other variants.

At both sites, the variants differed significantly regarding the root mass remaining in the soil. Amaranth, maize and partly the flowering mixtures had very low root dry masses with no more than $1 \mathrm{t} \mathrm{DM} \mathrm{ha-1}$. In contrast, the summer and winter faba beans but also the intercropping of winter faba bean with winter triticale as well as the ryegrass were able to produce high root dry matter yields of up to $6 \mathrm{t} \mathrm{DM} \mathrm{ha}{ }^{-1}$.

The cultivation of non-legume or grain-dominant first crops in the double cropping system partially led to a decrease in Nmin in winter crops compared to the bare fallow. At site Reinshof, the winter faba bean did not differ from the bare fallow. At site Schoningen, the effects of the first crops on the Nmin were not so clear over winter. At both sites the perennial species, i.e. ryegrass, the perennial flowering mixture and cup plant, were able to keep Nmin low over winter. After harvesting, maize as the second crop showed the highest residual $\mathrm{N}$-quantities. This was eminently true for maize after winter faba beans.

Large differences between the experimental sites Reinshof and Schoningen, but also between the experimental years, became apparent in the soil water content after the crops were harvested. In the first experimental year, the first crops in the double cropping system drained the soil until the harvest in early June, while in the second experimental year, at least at site Reinshof, the soil water content did not differ significantly from the bare fallow. The variants that were harvested during the summer months tended to leave less water in the soil than the variants that were harvested in autumn. At the Schoningen site, temporarily there was no plant available water in the soil at all during the first experimental year. The experimental years differed extremely on this point. It was therefore difficult to determine which differences in the soil water content were caused by the crops themselves.

The survey in the human population submitted a clear picture over the acceptance of energy crops for biogas production. The vast majority agreed to the cultivation of flower mixtures. The cup plant as a flowering variant, however, performed less well. The least accepted variants were amaranth, the intercropping of maize with amaranth and the maize in pure stand as a main crop.

From an economic perspective, the maize at the Reinshof site performed very well with contribution margins of up to $1400 €$ ha $^{-1}$. In the second experimental year, however, the double cropping systems and the cup plant were able to keep up. The contribution margins of amaranth, the annual and perennial flowering mixture and the summer faba bean with values between 400 and a maximum of $700 €$ ha $^{-1}$ were significantly lower. At site Schoningen, the contribution margins were at an overall lower level. In the case of the annual flowering mixture from the second experimental year, the costs could not be covered by the revenues. At this location, maize led to a comparatively good economic result. But the ryegrass crop having a contribution margin of $994 €$ ha $^{-1}$ even exceeded the maize crop with $824 € \mathrm{ha}^{-1}$ in the second experimental year. 
By means of the sub-indices calculated from the presented results, the crops could be evaluated relatively to each other. Advantages and disadvantages of the individual crops became visible. The aggregation of the sub-indices to form the overall index IrA finally enabled the creation of a ranking list depending on the relative suitability. It was shown that the ryegrass crop was the most suitable variant at both sites, based on the nine-stage evaluation scheme. At the site Schoningen, this variant combined almost all sub-indices in the positive range and thus stood out by far from the other variants. At site Reinshof, the difference in the index IrA between the ryegrass and the variants of the double cropping system was not quite as large. The cup plant also achieved a positive IrA value at both sites and can therefore be recommended as an energy crop for biogas production in addition to ryegrass and at site Reinshof to the double cropping systems.

Given the fact of the best possible trace element supply for the biogas fermenter, the following variants, showing a positive IrA value, can be recommended: The ryegrass at both locations, the winter faba bean followed by maize at site Reinshof and the summer faba bean at site Schoningen. However, the yield performance of the summer faba bean must be improved substantially in the future.

The investigations have shown that with IrA and the associated sub-indices it is possible to adequately and transparently illustrate the relative agronomical suitability of a crop regarding biogas production. The IrA index is designed in such a way that it can easily be extended or adapted to special needs. 
Amelung, W., Blume, H.-P., Fleige, H., Horn, R., Kandeler, E., Kögel-Knabner, I., Kretzschmar, R., Stahr, K. \& B.-M. Wilke, 2018: Scheffer/Schachtschabel Lehrbuch der Bodenkunde. Springer Verlag, Berlin, 17. Auflage.

Anthes, J., 2005: Beitrag von Ackerbohne (Vicia faba L.), Luzerne (Medicago sativa L.) und Saatwicke (Vicia sativa L.) zur Selbstregelung der N-Zufuhr in leguminosenbasierten Fruchtfolgen. Dissertation, Georg-August-Universität Göttingen.

Aurbacher, J., Kornatz, P. \& J. Müller, 2018: Entwicklung und Vergleich von optimierten Anbausystemen für die landwirtschaftliche Produktion von Energiepflanzen unter den verschiedenen Standortbedingungen Deutschlands - Phase III. Ökonomische Begleitforschung. Abschlussbericht, Justus-Liebig-Universität Gießen.

Ayodele, O.J. \& O.S. Shittu, 2013: Fertilizer effects on biological efficiency of maize-leaf amaranth intercropping systems. International Research Journal of Agricultural Science and Soil Science 3, 81-87.

Bates, D., Maechler, M., Bolker, B., Walker, S., Christensen, R.H.B., Singmann, H., Dai, B., Scheipl, F. \& J. Fox, 2019: Package 'Ime4'. Linear Mixed-Effects Models using 'Eigen' and S4. Version 1.1-21.

Bauer, B. \& G. Guggenberger, 2020: Humusaufbau. Die Wissenschaft hinter den Legenden. DLG Mitteilungen, Heft 5, Sonderheft, $\mathrm{CO}_{2}$ speichern. Geschäftsmodell Klimawirt. 12-16.

Baumgärtel, G. \& T. Eiler, 2012: Mais bedarfsgerecht düngen. Zeitpunkt des höchsten N-Bedarfs und hoher Bodennachlieferung ist deckungsgleich. Mais, Heft 1, 8-13.

Becker, H., 2011: Pflanzenzüchtung. Verlag Eugen Ulmer, Stuttgart, 2. Auflage.

Biertümpfel, A. \& M. Conrad, 2013: Erhöhung des Leistungspotenzials und der Konkurrenzfähigkeit der Durchwachsenen Silphie als Energiepflanze durch Züchtung und Optimierung des Anbauverfahrens. Teilvorhaben 2: Optimierung des Anbauverfahrens und Bereitstellung von Selektionsmaterial. Abschlussbericht, Thüringer Landesanstalt für Landwirtschaft, Jena.

BMEL, 2018: Änderungen bei den Direktzahlungen ab dem Antragsjahr 2018. https://www.bmel.de/SharedDocs/Downloads/DE/_Landwirtschaft/EU-Agrarpolitik-Foerderung/AendDirektzahlungen2018. pdf?blob=publicationFile\&v=5, besucht am 30. Mai 2020.

BNatSchG, 2009: Bundesnaturschutzgesetz von 2009. Gesetz über Naturschutz und Landschaftspflege. Änderung des BNatSchG vom 19. Juni 2020.

Borreani, G., Revello Chion, A., Colombini, S., Odoardi, M., Paoletti, R. \& E. Tabacco, 2009: Fermentative profiles of field pea (Pisum sativum), faba bean (Vicia faba) and white lupin ( $\mathrm{Lu}$ pinus albus) silages as affected by wilting and inoculation. Animal Feed Science and Technology 151, 316-323.

Breitschuh, G., Eckert, H., Matthes, I. \& J. Strümpfel, 2008: Kriteriensystem nachhaltige Landwirtschaft. KTBL-Schrift 466. 
Brock, C., Bernert, S., Willms, M., Knebl, L \& G. Leithold, 2013: Bewertung von Energiepflanzen in der Humusbilanz - Status quo und Perspektiven. Journal für Kulturpflanzen 65, 227235.

Bruns, D. \& D. Münderlein, 2018: Gesundheitsfördernde Landschaften - Eine Betrachtung von Naturparken. In: Wohin des Weges? Regionalentwicklung in Großschutzgebieten. (Hrsg. F. Weber, F. Weber, C. Jenal), Arbeitsberichte der ARL 21, 250-281.

Büchter, M., Wachendorf, M. \& F. Taube, 2001: Nitratauswaschung unter Silomais in Abhängigkeit von der Bewirtschaftungsform und der N-Düngungsintensität - Ergebnisse aus dem NProjekt Karkendamm. Mitteilungen der Arbeitsgemeinschaft für Grünland und Futterbau 3, 7577.

Bundessortenamt, 2015: Blatt für Sortenwesen. Amtsblatt des Bundessortenamtes 48, Sonderheft, 1-117.

Cardinale, B.J., Duffy, J.E., Gonzalez, A., Hooper, D.U., Perrings, C., Venail, P., Narwani, A., Mace, G.M., Tilman, D., Wardle, D.A., Kinzig, A.P., Daily, G.C., Loreau, M., Grace, J.B., Larigauderie, A., Srivastava, D.S. \& S. Naeem, 2012: Biodiversity loss and its impact on humanity. Nature 489, 59-67.

Chatzistathis, T., 2018: Physiological importance of manganese, cobalt and nickel and the improvement of their uptake and utilization by plants. In: Plant Micronutrient Use Efficiency. (eds. M.A. Hossain, T. Kamiya, D. Burritt, L.-S. Phan Tran \& T. Fujiwara), Academic Press, London, 123-135.

Dabbert, S. \& J. Braun, 2006: Landwirtschaftliche Betriebslehre. Verlag Eugen Ulmer, Stuttgart.

Dauber, J., Muller, A.-L., Schittenhelm, S., Schoo, B., Schorpp, Q., Schrader, S. \& S. Schroetter, 2016: Agrarökologische Bewertung der Durchwachsenen Silphie (Silphium perfoliatum L.) als eine Biomassepflanze der Zukunft. Abschlussbericht, Johann Heinrich von ThünenInstitut und Julius-Kühn-Institut, Braunschweig.

de Leon-Gonzalez, F, Celada-Tornel, E., Hidalgo-Moreno, H.I., Etchevers-Barra, J.D., Gutierrez-Castorena, M.C. \& A. Flores-Macias, 2006: Root-soil adhesion as affected by crop species in a volcanic sandy soil of Mexico. Soil \& Tillage Research 90, 77-83.

Demirel, B. \& P. Scherer, 2011: Trace element requirements of agricultural biogas digesters during biological conversion of renewable biomass to methane. Biomass and Bioenergy 35, 992-998.

Dieckmann, C., Lamp, A., Schmidt, L.-M., Andersen, L., Baetge, S. \& M. Kaltschmitt, 2018: Von der Biogasanlage zur Bioraffinerie - Perspektiven für zukünftige Biogasanlagenkonzepte. Zeitschrift für Energiewirtschaft 42, 235-256.

DWD Climate Data Center (CDC), 2019: Tägliche Stationsmittelwerte für Temperatur und Niederschlag an den Wetterstationen Göttingen, Uslar und Wahlburg-Lippoldsberg. https://cdc.dwd.de/portal/, besucht am 5. September 2019. 
DWD, 2016: Dokumentation Bodenfeuchte. Deutscher Wetterdienst. https://www.dwd.de/DE/ fachnutzer/landwirtschaft/dokumentationen/allgemein/bf_erlaeuterungen.pdf?_blob=publicationFile\&v=7, besucht am 23. Juni 2020.

Eberl, V., Fahlbusch, W., Fritz, M. \& B. Sauer, 2014: Screening und Selektion von Amarantsorten und -linien als spurenelementreiches Biogassubstrat. Berichte aus dem Technologieund Förderzentrum (TFZ), Straubing, 37.

Eckner, J., Lindner, J. \& K. Winter, 2018: Entwicklung und Vergleich von optimierten Anbausystemen für die landwirtschaftliche Produktion von Energiepflanzen unter den verschiedenen Standortbedingungen Deutschlands - Phase III. Entwicklung und Optimierung von standortangepassten Anbausystemen für Energiepflanzen im Fruchtfolgeregime. Abschlussbericht, Thüringer Landesanstalt für Landwirtschaft, Jena.

Fahlbusch, W., Hey, K., Sauer, B. \& H. Ruppert, 2018: Trace element delivery for biogasproduction enhanced by alternative energy crops: results from two-year field trials. Energy, Sustainability and Society 8 , Article 38.

Fleschhut, M., 2015: Doppelernte beim Körnermais. Korn und Maisstroh. Innovation 4, 4-5.

Flessa, H., Greef, M., Hofmeier, M., Dittert, K., Ruser, R., Osterburg, B., Poddey, E., Wulf, S. \& A. Pacholski, 2014: Minderungen von Stickstoff-Emissionen aus der Landwirtschaft. Empfehlungen für die Praxis und aktuelle Fragen an die Wissenschaft. Forschung Themenheft 1, 2014.

FNR, Fachagentur Nachwachsende Rohstoffe, 2019: Basisdaten Bioenergie 2019. Gülzow.

FNR, Fachagentur Nachwachsende Rohstoffe, 2020: Anbau und Verwendung nachwachsender Rohstoffe in Deutschland. https://www.fnr-server.de/ftp/pdf/berichte/22004416.pdf, besucht am 29. Juli 2020.

Förster, L., Trauner, A., Eberl, V., Brunner, S., Neumann, L., Sauer, B. \& M. Fritz, 2019: Amarant als spurenelementreiches Biogassubstrat: Selektion zur Erarbeitung praxistauglicher Amarantlinien für bayerische Standorte. Abschlussbericht, Technologie und Förderzentrum im Kompetenzzentrum für Nachwachsende Rohstoffe, Straubing.

Franco, R.T., Buffiere, P \& R. Bayard, 2016: Ensiling for biogas production: Critical parameters. A review. Biomass and Bioenergy 94, 94-104.

Franzaring, J., Holz, I., Kauf, Z. \& A: Fangmeier; 2015: Responses of the novel bioenergy plant species Sida hermaphrodita (L.) Rusby and Silphium perfoliatum L. to $\mathrm{CO}_{2}$ fertilization at different temperatures and water supply. Biomass and Bioenergy 81, 574-583.

Fraser, P.M., Curtin, D., Harrison-Kirk, T., Meenken, E.D., Beare, M.H., Tabley, F., Gillespie, R.N. \& G.S. Francis, 2013: Winter nitrate leaching under different tillage and winter cover crop management practices. Soil Science Society of America Journal 77, 1391-1401.

Fuchs, J. \& N. Stockfisch, 2009: Effizienzentwicklung im Zuckerrübenanbau am Beispiel der N-Düngung. Zuckerindustrie 134, 33-41. 
Gál, J. Hursthouse, A., Tatner, P., Stewart, F. \& R. Welton, 2008: Cobalt and secondary poisoning in the terrestrial food chain: Data review and research gaps to support risk assessment. Environment International 34, 821-838.

Gansberger, M., Montgomery, L.F.R. \& P. Liebhard, 2015: Botanical characteristics, crop management and potential of Silphium perfoliatum $\mathrm{L}$. as a renewable resource for biogas production: A review. Industrial Crops and Products 63, 362-372.

Gensch, N., 2019: Die Mischung machts. DLG-Mitteilungen, Heft 7/2019, Sonderheft SaatgutMagazin.

Ghosheh, H.Z. \& M.K. El-Shatnawi, 2003: Broadleaf weed control in chickpea (Cicer arietinum), faba beans (Vicia faba) and lentils (Lens culinaris). Acta Agronomica Hungarica 51, 437-444.

Gimplinger, D.M. \& H.-P. Kaul, 2008: Calibration and validation of the crop growth model LINTUL for grain amaranth (Amaranthus sp.). Journal of Applied Botany and Food Quality 82, 183-192.

Graß, R., Heuser, F., Stülpnagel, R., Piepho, H.-P. \& M. Wachendorf, 2013: Energy crop production in double-cropping systems: Results from an experiment at seven sites. European Journal of Agronomy 51, 120-129.

Hartmann, A. \& T. Lunenberg, 2016: Ertragspotenzial der Durchwachsenen Silphie unter bayerischen Anbaubedingungen. Journal für Kulturpflanzen 68, 385-388.

Heidecker, B., 2008: Grundlagenerarbeitung zur Berechnung und Optimierung von Substratmischungen durch gezielte Nutzung von Synergieeffekten einzelner Einsatzstoffe. Abschlussbericht, Entwicklungs-, Beratungs- und Anwendungszentrum für die verstärkte Nutzung von Biomasse, Triesdorf.

Helffrich, D. \& H. Oechsner, 2003: Hohenheimer Biogasertragstest. Agrartechnische Forschung 9, Heft 3, 27-30.

Herbes, C., Jirka, E., Braun, J.P., Pukall. K., 2014: Der gesellschaftliche Diskurs um den „Maisdeckel" vor und nach der Novelle des Erneuerbare-Energien-Gesetzes (EEG) 2012. GAIA Ecological Perspectives for Science and Society 23, 100-108.

Herrmann, A., Messner, H. \& J. Rath, 2011: Mais (Zea mays). In: Lehrbuch des Pflanzenbaues. Band 2: Kulturpflanzen (Hrsg. N. Lütke Entrup \& B.C. Schäfer). AgroConcept Bonn, 457-488.

Herrmann, A. \& J. Rath, 2012: Biogas production from maize: Current state, challenges, and prospects. 1. Methane yield potential. Bioenergy Research 5, 1027-1042.

Herrmann, A., Budde, K., Kluß, C. \& F. Taube, 2018: Bringt der Zuchtfortschritt bei Silomais auch Vorteile für die C-Einträge in den Boden? 68. Öffentliche Hochschultagung "Landwirtschaft und Ernährung im Spannungsfeld zwischen Umwelt, Gesellschaft und Politik“, Tagungsband, 107-112. 
Hey, K., 2020: Treibhausgasminderung beim Anbau von Winterzwischenfrüchten und Dauerkulturen im Energiepflanzenbau. Forschungsprojekt (FKZ 22026317, Fachagentur Nachwachsende Rohstoffe), Georg-August-Universität Göttingen.

Hogrefe, G., 2020: Raps im Herbst - Jetzt wird es ernst. Land \& Forst, Heft 30, 21-22.

Hosseini, N.M., Palta, J.A., Berger, J.D. \& K.H.M. Siddique, 2009: Sowing soil water content effects on chickpea (Cicer arietinum L.): Seedling emergence and early growth interaction with genotype and seed size. Agricultural Water Management 96, 1732-1736.

Huth, E., Paltrinieria, S. \& J. Thiele, 2019: Bioenergy and its effects on landscape aesthetics A survey contrasting conventional and wild crop biomass production. Biomass and Bioenergy 122, 313-321.

IPCC, Intergovernmental Panel on Climate Change, 2019: Climate Change and Land. An IPCC special report on climate change, desertification, land degradation, sustainable land management, food security, and greenhouse gas fluxes in terrestrial ecosystems.

lqbal, N., Hussain, S., Ahmed, Z., Yang, F., Wang, X., Liu, W., Yong, T., Du, J., Shu, K., Yang, W. \& J. Liu, 2019: Comparative analysis of maize-soybean strip intercropping systems: a review. Plant Production Science 22, 131-142.

Johnson, B.L. \& T.L. Henderson, 2002: Water use patterns of grain amaranth in the Northern Great Plains. Agronomy Journal 94, 1437-1443.

Jost, B., 2003: Untersuchungen und Kalkulationstabellen zur Schätzung der $\mathrm{N}_{2}$-Fixierleistung und der N-Flächenbilanz beim Anbau von Lupinus albus und Lupinus luteus in Reinsaat und von Vicia faba und Pisum sativum in Reinsaat und Gemenge mit Avena sativa. Dissertation, Georg-August-Universität Göttingen.

Kabata-Pendias, A., 2011: Trace elements in soils and plants. CRC Press Taylor \& Francis, Boca Raton, 4. Auflage.

Kahnt-Ralle, E., 2017: Eine neue Chance für die Silphie? Land \& Forst, Heft 41, 28-29.

Kaiser, F. \& A. Gronauer, 2007: Methanproduktivität nachwachsender Rohstoffe in Biogasanlagen. Bayerische Landesanstalt für Landwirtschaft (LfL), Lerchl Druck, Freising.

Karpenstein-Machan, M. \& R. Stülpnagel, 2000: Biomass yield and nitrogen fixation of legumes monocropped and intercropped with rye and rotation effects on a subsequent maize crop. Plant and Soil 218, 215-232.

Karpenstein-Machan, M., 2005: Energiepflanzenbau für Biogasanlagenbetreiber. DLG Verlag, Frankfurt am Main.

Karpenstein-Machan, M. \& C. Weber, 2010: Energiepflanzenanbau für Biogasanlagen. Veränderungen in der Fruchtfolge und der Bewirtschaftung von Ackerflächen in Niedersachsen. Naturschutz und Landschaftsplanung 42, 312-320.

Karpenstein-Machan, M., 2017: Silphie: Mais der Zukunft? Energie aus Pflanzen 5, 56-59. 
Karsten, H.D. \& J.W. McAdam, 2001: Effect of drought on growth, carbohydrates, and soil water use by perennial ryegrass, tall fescue, and white clover. Crop Science 41, 156-166.

Kasper, M., Freyer, B., Hülsbergen, K.-J., Schmid, J., \& J.K. Friedel, 2015: Humus balances of different farm production systems in main production areas in Austria. Journal of Plant Nutrition and Soil Science 178, 25-34.

Kätterer, T., Anders Bolinder, M., Andrén, O., Kirchmann, H. \& L. Menichetti, 2011: Roots contribute more to refractory soil organic matter than above-ground crop residues, as revealed by a long-term field experiment. Agriculture, Ecosystems and Environment 141, 184-192.

Kauffman, C.S., \& L.E. Weber, 1990: Grain amaranth. In: Advances in new crops. (eds. J. Janick \& J.E. Simon), Timber Press, Portland, 127-139.

Kaul, H.-P., Aufhammer, W., Laible, B., Nalborczyk, E., Pirog, S. \& K. Wasiak, 1996: The suitability of amaranth genotypes for grain and fodder use in Central Europe. Die Bodenkultur 47, 173-181.

Koch, K., Gepperth, S., Andrade, D., Ebertseder, F. \& A. Gronauer, 2011: Hilfsmittel bei der Biogaserzeugung - Überblick und Erfahrungen aus Labor und Praxis. In: Einsatz von Hilfsmitteln zur Steigerung der Effizienz und Stabilität des Biogasprozesses. (Hrsg. Fachagentur Nachwachsende Rohstoffe), Gülzower Fachgespräche Band 35, 16-31.

Köpke, U. \& T. Nemecek, 2010: Ecological services of faba bean, Field Crops Research 115, 217-233.

KTBL, 2009: Faustzahlen für die Landwirtschaft. Kuratorium für Technik und Bauwesen in der Landwirtschaft, Darmstadt, 14. Auflage.

KTBL, 2012: Energiepflanzen. Daten für die Planung des Energiepflanzenanbaus. Darmstadt, 2. Auflage.

KTBL, 2013: Faustzahlen Biogas. Kuratorium für Technik und Bauwesen in der Landwirtschaft, Darmstadt.

Landesamt für Bergbau, Energie und Geologie, 2019: Bodenkarten für Niedersachsen. https://nibis.lbeg.de/cardomap3/, besucht am 20. August 2019.

Landesamt für Statistik Niedersachsen, 2019: Statistische Berichte. Bodennutzung und Ernte 2018.

Laser, H., 2019: Biomasseaufwertung und Silierung lignocellulosereicher Koppelprodukte zur Optimierung der Methanausbeute (BASiliKOM). Abschlussbericht, Fachhochschule Südwestfalen, Soest.

Laurenz, L., 2000: Mais: Mit Wärmesummen den Erntetermin bestimmen. top agrar, Heft 9, 70-73.

Lenth, R., Singmann, H., Love, J., Buerkner, P. \& M. Herve, 2019: Package 'emmeans'. Estimated Marginal Means, aka Least-Square Means. Version 1.3.3. 
Leonard Haag, N., Nägele, H-J, Reiss, K., Biertümpfel, A. \& H. Oechsner, 2015: Methane formation potential of cup plant (Silphium perfoliatum). Biomass and Bioenergy 75, 126-133.

LfL, Bayerische Landesanstalt für Landwirtschaft, 2020a: Biogasausbeuten-Datenbank. https://www.Ifl.bayern.de/iba/energie/049711/?sel_list=3\%2Cb\&anker0=substratanker\#substratanker, besucht am 27. Juli 2020.

LfL, Bayerische Landesanstalt für Landwirtschaft, 2020b: Zehn Jahre Stickstoff-Monitoring https://www.Ifl.bayern.de/iab/duengung/032228/index.php, besucht am 29. Juli 2020.

Li, L., Sun, J., Zhang, F., Li, X., Yang, S. \& Z. Rengel, 2001: Wheat/maize or wheat/soybean strip intercropping I. Yield advantage and interspecific interactions on nutrients. Field Crops Research 71, 123-137.

Li, L., Li, S.-M., Sun, J.-H., Zhou, L.-L., Bao, X.-G., Zhang, H.-G. \& F.-S. Zhang, 2007: Diversity enhances agricultural productivity via rhizosphere phosphorus facilitation on phosphorus-deficient soils. PNAS 104, 11192-11196.

Li, Z., McLaren, R.G. \& A.K. Metherell, 2004: The availability of native and applied soil cobalt to ryegrass in relation to soil cobalt and manganese status and other soil properties. New Zealand Journal of Agricultural Research 47, 33-43.

Lütke Entrup, N., 2011: Feldfutterbau und Ackerbegrünung. In: Lehrbuch des Pflanzenbaues. Band 2: Kulturpflanzen (Hrsg. N. Lütke Entrup \& B.C. Schäfer). AgroConcept Bonn, 657-760.

LWK Nordrhein-Westfalen, 2020: Übersicht ökologische Vorrangflächen. https://www.landwirtschaftskammer.de/foerderung/pdf/uebersicht-oekologische-vorrangflaechen.pdf, besucht am 30. Mai 2020.

Marschner, P., 2012: Marschner's Mineral Nutrition of Higher Plants. Academic Press, London, 3rd Edition.

Mast, B., Lemmer, A., Oechsner, H., Reinhardt-Hanisch, A., Claupein, W \& S. Graeff-Hönninger, 2014: Methane yield potential of novel perennial biogas crops influenced by harvest date. Industrial Crops and Products 58, 194-203.

Mau, K., 2019: Elektroautos: Dreckige Rohstoffe für saubere Autos. Zeit online. https://www.zeit.de/mobilitaet/2019-11/elektroautos-kobalt-lithium-batterie-akkus-rohstoffeumweltschutz, besucht am 11. August 2020.

Mayring, P., 2010: Qualitative Inhaltsanalyse. Belz Verlag, Weinheim, Basel, 12. Auflage.

Meinen, C., Rauber, R. \& S. Siebert, 2019: Hafer als Zwischenfrucht vermindert Nitratauswaschung im Winterackerbohnenanbau. Mitteilungen Gesellschaft für Pflanzenbauwissenschaften $31,81-82$.

Meisinger, J.J. \& K.A. Ricigliano, 2017: Nitrate leaching from winter cereal cover crops using undisturbed soil-column lysimeters. Journal of Environmental Quality 46, 576-584.

Mengel, K., 1991: Ernährung und Stoffwechsel der Pflanze. Gustav Fischer Verlag, Jena, 7. Auflage. 
Menke, C.A., 2011: Evaluierung von Winterzwischenfrüchten in einem Zweikultur-Nutzungssystem mit Mais für die Biogaserzeugung. Dissertation, Georg-August-Universität Göttingen, Cuvillier Verlag, Göttingen.

Menke, C.A. \& R. Rauber, 2015: Evaluierung von Winterzwischenfrüchten vor Mais zur Biogaserzeugung. Berichte über Landwirtschaft 93, Ausgabe 3.

Nassab, A.D.M., Amon, T. \& H.-P. Kaul, 2011: Competition and yield in intercrops of maize and sunflower for biogas. Industrial Crops and Products 34, 1203-1211.

Neugschwandtner, R., Ziegler, K., Kriegner, S., Wagentristl, H. \& H.-P. Kaul, 2015: Nitrogen yield and nitrogen fixation of winter faba beans. Acta Agriculturae Scandinavica, Section B Soil \& Plant Science 65, 658-666.

New Holland Agriculture, 2020: https://agriculture.newholland.com/eu/en-uk/about-us/whatson/news-events/2019/new-holland-unveils-the-first-t6-methan-power-tractor, besucht am 30 . Juli 2020.

Pahlow, G., 2003: Konservierung von Futterleguminosen. Mitteilungen Arbeitsgemeinschaft Grünland und Futterbau 5, 23-29.

Palit, S. \& A. Scharma, 1994: Effects of cobalt on plants. The Botanical Review 60, 149-181.

Pasold, T., 2020: Charakterisierung minimaler und maximaler Hemmkonzentrationen verschiedener Spurenelemente in Biogasprozessen und Entwicklung eines vereinfachten Bestimmungsverfahrens. Dissertation, Georg-August-Universität Göttingen.

Petersen, J., 2020: Weidelgras - der schlimmere Ackerfuchsschwanz. Getreide-Magazin, Heft 4, 24-27.

Petersson, A., Thomsen, M.H., Hauggaard-Nielsen, H. \& A.-B. Thomsen, 2007: Potential bioethanol and biogas production using lignocellulosic biomass from winter rye, oilseed rape and faba bean. Biomass and Bioenergy 31, 812-819.

Pichard, G., 2012: Management, production, and nutritional characteristics of cup-plant (Silphium perfoliatum) in temperate climates of southern Chile. Ciencia e Investigacion Agraria 39, 61-77.

Pobeheim, H., Munk, B., Lindorfer, H. \& G. Guebitz, 2011: Impact of nickel and cobalt on biogas production and process stability during semi-continuous anaerobic fermentation of a model substrate for maize silage. Water Research 45, 781-787.

R Core Team, 2019: R: A language and environment for statistical computing. R Foundation for Statistical Computing. Vienna, Austria. Version 3.5.3.

Raseduzzaman, M. \& E.S. Jensen, 2017: Does intercropping enhance yield stability in arable crop production? A meta-analysis. European Journal of Agronomy 91, 25-33.

Rauber, R., Schmidtke, K. \& H. Kimpel-Freund, 2001: The performance of pea (Pisum sativum L.) and its role in determining yield advantages in mixed stands of pea and oat (Avena sativa L.) Journal of Agronomy \& Crop Science 187, 137-144. 
Renius, W., Lütke Entrup, E. \& N. Lütke Entrup, 1992: Zwischenfruchtbau zur Futtergewinnung und Gründüngung. DLG Verlag, Frankfurt am Main, 3. Auflage.

Roth, F., 2010: Evaluierung von Winterackerbohnen als Zwischenfrucht für eine ökologische Biogasproduktion. Dissertation, Georg-August-Universität Göttingen.

Sauer, B., 2010: Elementgehalte und Stoffströme bei der Strom- und Wärmegewinnung im Bioenergiedorf Jühnde. Dissertation Georg-August-Universität Göttingen. Schriftenreihe Fortschritt neu denken, Heft 2, Göttingen.

Sauer, B., Fahlbusch, W. \& H. Ruppert, 2014: Möglichkeiten einer ausgeglichenen Spurenelementversorgung von Biogasanlagen durch Mischungen unterschiedlicher Energiepflanzen. Abschlussbericht, Interdisziplinäres Zentrum für Nachhaltige Entwicklung Universität Göttingen.

Sauer, B., Sträuber, H., Fahlbusch, W, Hey, K., Pasold, T., Pröter, J., Rauber, R, Ruppert, H. \& Zechendorf, M, 2018: Spurenelemente durch Energiepflanzen - Stoffströme und Handlungsempfehlungen für eine optimierte Prozessbiologie in Biogasanlagen (NiCo). Abschlussbericht, Georg-August-Universität, Göttingen.

Sauerbeck, G., 2004: Amaranth - Ergänzungspflanze für Mischsilagen mit Mais? Mitteilungen Gesellschaft für Pflanzenbauwissenschaften 16, 35-36.

Schäfer, B.C., 2011: Körnerleguminosen. In: Lehrbuch des Pflanzenbaues. Band 2: Kulturpflanzen (Hrsg. N. Lütke Entrup \& B.C. Schäfer). AgroConcept Bonn, 626-656.

Schittenhelm, S., Reus, D., Kruse, S. \& J. Hufnagel, 2011: Assessment of productivity and profitability of sole and double-cropping for agricultural biomass production. Journal für Kulturpflanzen 63, 387-395.

Schnürer, A., 2016: Biogas production: microbiology and technology. In: Anaerobes in Biotechnology. Advances in Biochemical Engineering/Biotechnology 256. (eds. R. Hatti-Kaul, G. Mamo \& B. Mattiasson), Springer, Cham, 195-234.

Schoo, B., Schroetter, S., Kage, H. \& S. Schittenhelm, 2017: Root traits of cup plant, maize and lucerne grass grown under different soil and soil moisture conditions. Journal of Agronomy and Crop Science 203, 345-359.

Schoo, B., Wittich, K.P., Böttcher, U., Kage, H. \& S. Schittenhelm, 2017: Drought tolerance and water-use efficiency of biogas crops: A comparison of cup plant, maize and lucerne-grass. Journal of Agronomy and Crop Science 203, 117-130.

Schorpp, Q., Müller, A.L., Schrader, S. \& J. Dauber, 2016: Agrarökologisches Potential der Durchwachsenen Silphie (Silphium perfoliatum L.) aus Sicht biologischer Vielfalt. Journal für Kulturpflanzen 68, 412-422.

Schüpbach, B., Junge, X., Briegel, R., Lindemann-Matthies, P. \& T. Walter, 2009: Ästhetische Bewertung landwirtschaftlicher Kulturen durch die Bevölkerung. Schriftenreihe Forschungsanstalt Agroscope Reckenholz-Tänikon 10, 1-67. 
Seitz, D. \& C. Drewes, 2019: Kooperation Trinkwasserschutz. Herbst-Nmin-Gehalte. Geries Ingenieure, Information 5/2019.

Siaudinis, G., Jasinskas, A., Sarauskis, E., Steponavicius, D., Karcauskiene, D. \& I. Liaudanskiene, 2017: The assessment of Virginia mallow (Sida hermaphrodita Rusby) and cup plant (Silphium perfoliatum L.) productivity, physico-mechanical properties and energy expenses. Energy 93, 606-612.

Siebrecht-Schöll, D.J., 2019: Züchterische Analyse von acht Winterackerbohnengenotypen für den Gemengeanbau mit Winterweizen. Dissertation, Georg-August-Universität Göttingen.

Sieling, K., Herrmann, A., Wienforth, B., Taube, F., Ohl, S., Hartung, E. \& H. Kage, 2013: Biogas cropping systems: Short term response of yield performance and $\mathrm{N}$ use efficiency to biogas residue application. European Journal of Agronomy 47, 44-54.

Statistisches Bundesamt, 2005: Land- und Forstwirtschaft, Fischerei. Wachstum und Ernte Feldfrüchte 2004. Destatis Fachserie 3, Reihe 3.2.1.

Statistisches Bundesamt, 2020: Land- und Forstwirtschaft, Fischerei. Wachstum und Ernte Feldfrüchte 2019. Destatis Fachserie 3, Reihe 3.2.1.

Stinner, P.W., Deuker, A., Schmalfuß, T., Brock, C., Rensberg, N., Denysenko, V., Trainer, P., Möller, K., Zang, J., Janke, L., Mozena Leandro, W., Oehmichen, K., Popp, D. \& J. DanielGromke, 2018: Perennial and intercrop legumes as energy crops for biogas production. In: Legumes for Soil Health and Sustainable Management. (ed. R.S. Meena), Springer Nature, Singapore, 139-170.

Streit, J., 2018: Biomass, root distribution and overyielding potential of faba bean/wheat and white clover/ryegrass mixtures. Dissertation, Georg-August-Universität Göttingen.

Streit, J., Meinen, C., Nelson, W.C.D., Siebrecht-Schöll D.J. \& R. Rauber, 2019: Above- and belowground biomass in a mixed cropping system with eight novel winter faba bean genotypes and winter wheat using FTIR spectroscopy for root species discrimination. Plant and Soil 436, $141-158$.

Stülpnagel, R., von Buttlar, C., Heuser, F. \& M. Wachendorf, 2009: Entwicklung und Vergleich von optimierten Anbausystemen für die landwirtschaftliche Produktion von Energiepflanzen unter den verschiedenen Standortbedingungen Deutschlands. Systemversuch zum Zweikultur-Nutzungssystem auf sechs Standorten im Bundesgebiet. Abschlussbericht, Universität Kassel.

Techow, A., Quakernack, R., Pacholski, A., Kage, H., Taube, F., \& A. Herrmann, 2011: Perennial ryegrass for biogas production: How do cutting frequency and cultivar maturity influence methane yield? Grassland Science in Europe 16, 377-379.

Thapa, R., Mirsky, S.B. \& K.L. Tully, 2018: Cover crops reduce nitrate leaching in agroecosystems: A global meta-analysis. Journal of Environmental Quality 47, 1400-1411.

Tobi, D., Herrmann, A., Loges, R., Hasler, M., Gierus, M. \& F. Taube, 2011: Effect of heading date, ploidy level and cutting regime on yield and feed quality of perennial ryegrass (Lolium perenne L.). Grassland Science in Europe 16, 184-186. 
Toews, T., Stülpnagel, R. \& C. von Buttlar, 2008: Mit dem Zweifrucht-System den Gewinn erhöhen. top agrar, Heft 6, 88-60.

Ugilt Larsen, S., Hjort-Gregersen, K., Heidarzadeh Vazifehkhoran, A. \& J.M. Triolo, 2017: Coensiling of straw with sugar beet leaves increases the methane yield from straw. Bioresource Technology 245, 106-115.

VDLUFA, 2014: Standpunkt Humusbilanzierung. Eine Methode zur Analyse und Bewertung der Humusversorgung von Ackerland. Verband Deutscher Landwirtschaftlicher Untersuchungs- und Forschungsanstalten, Speyer.

Vetter, A., 2009: Anbausysteme für Energiepflanzen. In: Anbausysteme für Energiepflanzen optimierte Fruchtfolgen und effiziente Lösungen. (Hrsg. A. Vetter, M. Heiermann, T. Toews), DLG-Verlag, 109-187.

Völkel, G. \& K. Schmidtke, 2019: Bodenbearbeitung zur Körnerleguminose und zur Folgefrucht. In: Körnerleguminosen anbauen und verwerten (Hrsg. Kuratorium für Technik und Bauwesen in der Landwirtschaft, KTBL). KTBL-Heft 100, 10-12.

Vollrath, B., 2012: Energetische Verwertung von kräuterreichen Ansaaten in der Agrarlandschaft und im Siedlungsbereich - eine ökologische und wirtschaftliche Alternative bei der Biogasproduktion. Abschlussbericht, Bayerische Landesanstalt für Weinbau und Gartenbau, Veitshöchheim.

von Bredow, H., 2010: Rechtliche und administrative Rahmenbedingungen. In: Leitfaden Biogas. (Hrsg. Fachagentur Nachwachsende Rohstoffe, FNR), 144-171.

von Cossel, M. \& I. Lewandowski, 2016: Perennial wild plant mixtures for biomass production: Impact of species composition dynamics on yield performance over a five-year cultivation period in southwest Germany. European Journal of Agronomy 79, 74-89.

von Cossel, M., Möhring, J., Kiesel, A. \& I. Lewandowski, 2017: Methane yield performance of amaranth (Amaranthus hypochondriacus L.) and its suitability for legume intercropping in comparison to maize (Zea mays L.). Industrial Crops \& Products 103, 107-121.

von Cossel, M., Steberl, K., Hartung, J., Pereira, L.A., Kiesel, A. \& I. Lewandowski, 2019: Methane yield and species diversity dynamics of perennial wild plant mixtures established alone, under cover crop maize (Zea mays L.), and after spring barley (Hordeum vulgare L.). GCB Bioenergy 11, 1376-1391.

Weiland, P, 2010: Biogas production: current state and perspectives. Applied Microbiology and Biotechnology 85, 849-860.

Wiehe, J., von Ruschkowski, E., Rode, M., Kanning, H. \& C. von Haaren, 2009: Auswirkungen des Energiepflanzenanbaus auf die Landschaft - Am Beispiel des Maisanbaus für die Biogasproduktion in Niedersachsen. Naturschutz und Landschaftsplanung 41, 107-113.

Yang, X.E., Baligar, V.C., Foster, J.C. \& D.C. Martens, 1997: Accumulation and transport of nickel in relation to organic acids in ryegrass and maize grown with different nickel levels. Plant and Soil 196, 271-276. 
Yu, Y., Stomph, T.-J., Makowski, D., Zhang, L. \& W. van der Werf, 2016: A meta-analysis of relative crop yields in cereal/legume mixtures suggests options for management. Field Crops Research 198, 269-279.

Zander, D., Eberl, V. Fritz, M., Lohr, H \& K. Deiglmayr, 2018: Wickroggen als Biogassubstrat. Biogas Forum Bayern. https://www.biogas-forum-bayern.de/media/files/0004/wickroggen-alsbiogassubstrat.pdf, besucht am 15. Dezember 2019. 


\section{Anhang}

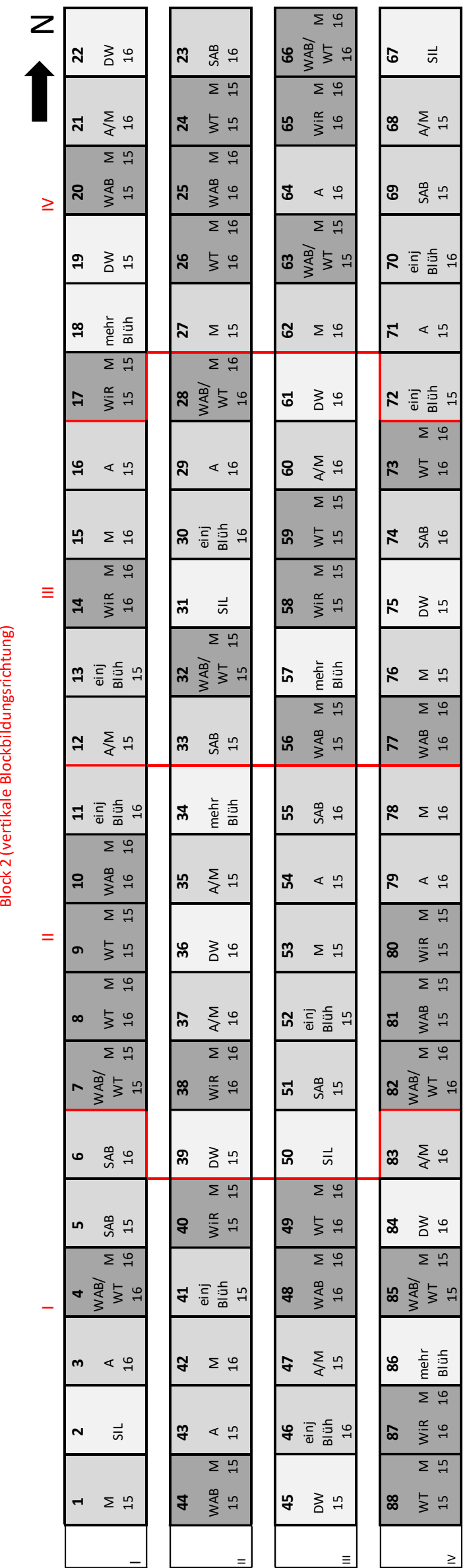

Abbildung A1: Versuchsplan Standort Reinshof für die beiden Versuchsjahre 2015 und 2016.

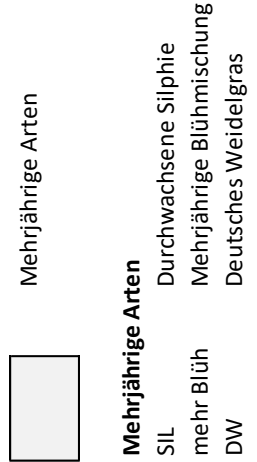

ह
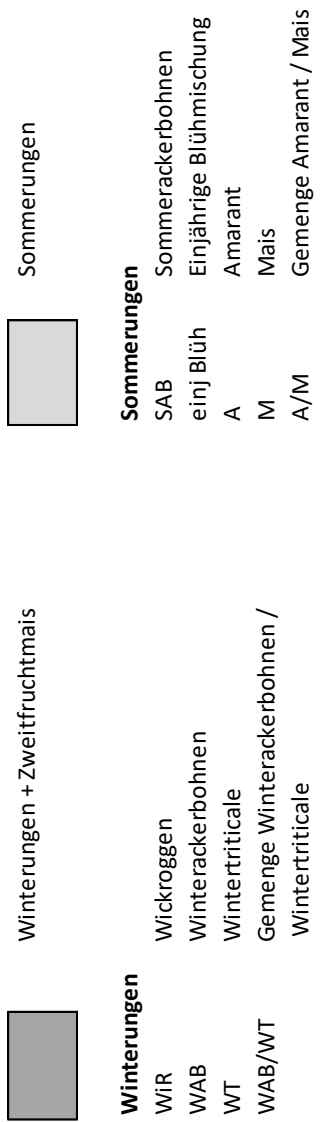


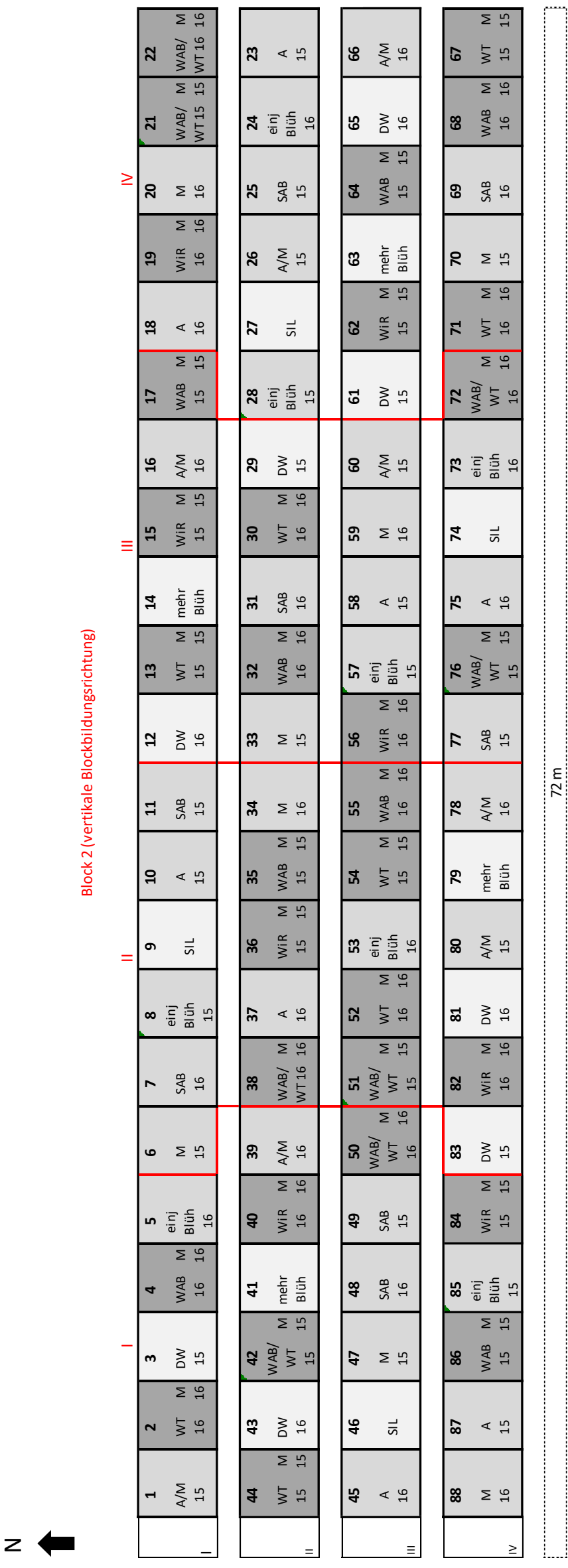

Abbildung A2: Versuchsplan Standort Schoningen für die beiden Versuchsjahre 2015 und 2016.
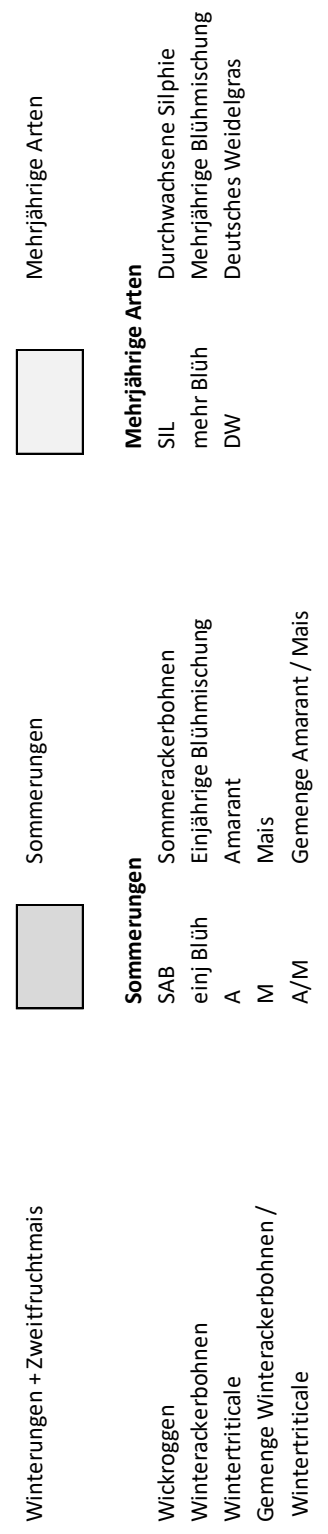

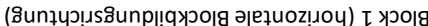


Tabelle A1: Zusammensetzung des Saatgutes der mehrjährigen Blühmischung (BG70) mit ein- und mehrjährigen Arten, sortiert nach Gewichtsanteil der Arten in der Mischung.

\begin{tabular}{llc}
\hline Deutsche Bezeichnung & Botanische Bezeichnung & $\begin{array}{c}\text { Anteil } \\
\text { (Gew.-\%) }\end{array}$ \\
\hline Gelber Steinklee & Melilotus officinalis & 10,0 \\
Buchweizen & Fagopyrum esculentum & 8,0 \\
Sonnenblume (einköpfig) & Helianthus annuus & 8,0 \\
Esparsette & Onobrychis viciifolia & 8,0 \\
Quirlmalve & Malva verticillata & 7,5 \\
Eibisch & Althaea officinalis & 7,5 \\
Flockenblume & Centaurea jacea/nigra & 7,0 \\
Weißer Steinklee (Wildform, 2-jährig) & Melilotus albus & 6,5 \\
Futtermalve & Malva sylvestris & 6,0 \\
Weißer Steinklee (Sorte Krajova, 2-jährig) & Melilotus albus & 5,0 \\
Weißer Steinklee (Sorte Adela, 1-jährig) & Melilotus albus & 5,0 \\
Rainfarn & Tanacetum vulgare & 5,0 \\
Fenchel & Foeniculum vulgare & 3,5 \\
Alant & Inula helenium & 3,5 \\
Luzerne & Medicago sativa & 2,0 \\
Färberkamille & Anthemis tinctoria & 1,5 \\
Wegwarte & Cichorium intybus & 1,5 \\
Weiße Lichtnelke & Silene latifolia & 1,0 \\
Rote Lichtnelke & Silene dioica & 1,0 \\
Beifuß & Artemisia vulgaris & 0,5 \\
Wilde Möhre & Daucus carota & 0,5 \\
Wilde Karde & Dipsacus fullonum & 0,5 \\
Natternkopf & Echium vulgare & 0,5 \\
Färber-Wau & Reseda luteola & 0,3 \\
Königskerze & Verbascum spec. & 0,2 \\
\hline &
\end{tabular}


Tabelle A2: Zusammensetzung des Saatgutes der einjährigen Blühmischung (BG80), sortiert nach Gewichtsanteil der Arten in der Mischung.

\begin{tabular}{llc}
\hline Deutsche Bezeichnung & Botanische Bezeichnung & $\begin{array}{c}\text { Anteil } \\
\text { (Gew.-\%) }\end{array}$ \\
\hline Weißer Steinklee & Melilotus albus & 20,0 \\
Sonnenblume & Helianthus annuus & 15,0 \\
Quirlmalve & Malva verticillata & 15,0 \\
Wilde Malve & Malva sylvestris & 11,0 \\
Gelber Steinklee & Melilotus officinalis & 10,0 \\
Ramtillkraut & Guizotia abyssinica & 8,0 \\
Öllein & Linum usitatissimum & 5,0 \\
Buchweizen & Fagopyrum esculentum & 4,0 \\
Schmuckkörbchen & Cosmos bipinnatus & 4,0 \\
Ringelblume & Calendula officinalis & 4,0 \\
Bischofskraut & Ammi visnaga & 2,0 \\
Bechermalve & Lavatera trimestris & 2,0 \\
\hline
\end{tabular}


Tabelle A3: Deckungsbeitragsrechnung am Beispiel von Mais am Standort Reinshof im Versuchsjahr 2016.

\begin{tabular}{|c|c|c|c|c|}
\hline Leistungen & Me & nge & Preis & Betrag \\
\hline Mais GP: FM 69 t/ha, TS 34,4 \% & 23,8 & t/ha TM & $75,0 € / \mathrm{t} \mathrm{TM}$ & $1785,0 € / \mathrm{ha}$ \\
\hline spez. Methanertrag & 0,356 & $\begin{array}{l}\mathrm{Nm}^{3} \mathrm{CH} 4 / \\
\mathrm{kg} \text { oTS }\end{array}$ & & \\
\hline Wert Gärrest & 51,0 & $\mathrm{~m}^{3}$ & $7,0 € / \mathrm{m}^{3}$ & $357,0 € /$ ha \\
\hline Summe Leistungen & & & & $2142,0 € /$ ha \\
\hline Variable Kosten & Me & nge & Preis & Betrag \\
\hline Mais, Hybrid-Saatgut & 2,04 & U/ha & $106,0 € / U$ & $216,2 € /$ ha \\
\hline KAS $(27 \% \mathrm{~N})$, lose, $103 \mathrm{~kg} \mathrm{~N} / \mathrm{ha}$ & 381,5 & $\mathrm{~kg} / \mathrm{ha}$ & $0,29 € / \mathrm{kg}$ & $110,6 € /$ ha \\
\hline Kohlensaurer Kalk & 1,0 & t/ha & $27,0 € / \mathrm{t}$ & $27,0 € /$ ha \\
\hline Gärrest Biogasanlage & 20,8 & $\mathrm{~m}^{3} / \mathrm{ha}$ & $2,0 € / \mathrm{m}^{3}$ & $41,7 € /$ ha \\
\hline Herbizid, Intensitätsstufe 1 & 300 & l/ha & & $78,0 € /$ ha \\
\hline Wasser & 0,3 & $\mathrm{~m}^{3} / \mathrm{ha}$ & $1,8 € / \mathrm{m}^{3}$ & $0,5 € /$ ha \\
\hline Hagelversicherung* & 1920,0 & $€ /$ ha & $7,4 € / 1000 €$ & $14,2 € /$ ha \\
\hline Zinskosten (3 Monate) & 122,1 & $€ /$ ha & $0,03 € / €$ & $3,7 € /$ ha \\
\hline Grubber, $102 \mathrm{KW} \mathrm{AB} \mathrm{3-4,5} \mathrm{m}$ & & & & $20,0 € /$ ha \\
\hline Kreiselegge, $67 \mathrm{KW}, 3 \mathrm{~m}$ & & & & $19,0 € /$ ha \\
\hline Einzelkorn-Sämaschine, 54 KW, 6 m & 8,0 & Reihen & & $11,8 € /$ ha \\
\hline Düngen Anbauschl.-streuer $1,5 \mathrm{~m}^{3}, 24 \mathrm{~m}$ & 381,5 & $\mathrm{~kg} / \mathrm{ha}$ & & $1,2 € /$ ha \\
\hline Gärrestausbringung $15 \mathrm{~m}$ & 19,0 & $\mathrm{~m}^{3} / \mathrm{ha}$ & $4,5 € / \mathrm{m}^{3}$ & $85,5 € /$ ha \\
\hline Hacken $6 \mathrm{~m}$ & 8,0 & Reihen & & $8,4 € /$ ha \\
\hline PSM-Spritze angehängt 3000 I, 24 m & 300 & I/ha & $2,0 € /$ ha & $2,0 € /$ ha \\
\hline Ernte Häckseln/Transport & & & & $104,2 € /$ ha \\
\hline Kalkstreuer & & & & $6,0 € /$ ha \\
\hline Stoppelbearbeitung & & & & $12,9 € /$ ha \\
\hline Zinskosten (3 Monate) & 67,8 & $€ /$ ha & $0,03 € / €$ & $2,0 \in /$ ha \\
\hline Summe variable Kosten & & & & $765,0 € /$ ha \\
\hline Deckungsbeitrag I & & & & $1377,0 € / \mathrm{ha}$ \\
\hline
\end{tabular}

Annahme: Silomais (Biogasproduktion), wendend, gezogene Saatbettbereitung, Saat, konventionell/integriert, Schlaggröße 20 ha, Ertragsniveau hoch, mittlerer Boden, 102-kW-Mechanisierung (eigen), Hof-Feld-Entfernung $2 \mathrm{~km}$. $U=$ Einheit (Unit), $A B=$ Arbeitsbreite. Deckungsbeitrag $\mathrm{I}=$ Leistung abzüglich variable Kosten. * Hagelversicherung: Versicherungssumme je Hektar von $1920 €$ bei Totalausfall, Versicherungsprämie von $7,40 €$ je $1000 €$ Versicherungssumme. 
Tabelle A4: Zusammensetzung des Teilindex Nmin als Mittelwert aus Nmin (w), erhoben im Winter und Nmin (s/h), erhoben im Sommer bzw. Herbst nach der Ernte in den Versuchsjahren 2015 und 2016 a) am Standort Reinshof und b) am Standort Schoningen. Bei den Varianten des Zweikulturnutzungssystems (Wickroggen, Winterackerbohnen, Wintertriticale und Gemenge Winterackerbohnen/Wintertriticale) wurde der Nmin-Wert im Winter bei Winterungen und nach der Ernte nach der Zweitfrucht Mais ermittelt. Bei den Sommerhauptfrüchten (Sommerackerbohnen, Einjährige Blühmischung, Amarant, Mais und Gemenge Amarant/Mais) wurde der Nmin-Wert im Winter bei Schwarzbrache erhoben. Bei den mehrjährigen Arten (Deutsches Weidelgras, Mehrjährige Blühmischung, Durchwachsene Silphie) stellt das erste Versuchsjahr das Ansaat- bzw. Pflanzjahr dar.

\begin{tabular}{ccccccc}
\hline & 2015 & & \multicolumn{2}{c}{2016} \\
\hline & $\begin{array}{c}\text { Nmin } \\
(\mathrm{w})\end{array}$ & $\begin{array}{c}\text { Nmin } \\
(\mathrm{s} / \mathrm{h})\end{array}$ & $\begin{array}{c}\text { Teilindex } \\
\text { Nmin }\end{array}$ & $\begin{array}{c}\text { Nmin } \\
\text { (w) }\end{array}$ & $\begin{array}{c}\text { Nmin } \\
(\mathrm{s} / \mathrm{h})\end{array}$ & $\begin{array}{c}\text { Teilindex } \\
\text { Nmin }\end{array}$ \\
\hline
\end{tabular}

a)

Reinshof

\begin{tabular}{lcccccc}
\hline Wickroggen + Mais & 0,706 & $-0,186$ & 0,260 & 0,003 & $-0,514$ & $-0,256$ \\
Winterackerbohnen (WAB) + Mais & $-0,080$ & $-1,568$ & $-0,824$ & $-0,300$ & $-1,017$ & $-0,659$ \\
Wintertriticale (WT) + Mais & 0,756 & $-0,359$ & 0,198 & 0,033 & $-0,336$ & $-0,152$ \\
Gemenge WAB/WT + Mais & 0,466 & $-0,359$ & 0,053 & 0,078 & $-0,433$ & $-0,177$ \\
Sommerackerbohnen & $-0,306$ & $-0,476$ & $-0,391$ & $-0,323$ & $-0,139$ & $-0,231$ \\
Einjährige Blühmischung & $-0,306$ & 0,729 & 0,212 & $-0,323$ & 0,664 & 0,170 \\
Amarant & $-0,306$ & 0,708 & 0,201 & $-0,323$ & 0,720 & 0,199 \\
Mais & $-0,306$ & $-0,027$ & $-0,166$ & $-0,323$ & $-0,631$ & $-0,477$ \\
Gemenge Amarant/Mais & $-0,306$ & 0,292 & $-0,007$ & $-0,323$ & $-0,577$ & $-0,450$ \\
Deutsches Weidelgras & $-0,011$ & 0,804 & 0,397 & 0,733 & 0,867 & 0,800 \\
Mehrjährige Blühmischung & $-0,306$ & 0,441 & 0,068 & 0,530 & 0,680 & 0,605 \\
Durchwachsene Silphie & n.v. & n.v. & n.v. & 0,538 & 0,714 & 0,626
\end{tabular}

b)

\begin{tabular}{lcccccc}
\hline Wickroggen + Mais & n.v. & n.v. & n.v. & -0.203 & -0.307 & -0.255 \\
Winterackerbohnen (WAB) + Mais & $-0,091$ & $-1,249$ & $-0,670$ & 0,043 & $-1,616$ & $-0,786$ \\
Wintertriticale (WT) + Mais & 0,084 & $-0,383$ & $-0,149$ & 0,171 & $-0,897$ & $-0,363$ \\
Gemenge WAB/WT + Mais & 0,020 & $-0,345$ & $-0,162$ & 0,147 & $-0,642$ & $-0,247$ \\
Sommerackerbohnen & $-0,002$ & $-0,411$ & $-0,207$ & $-0,254$ & 0,372 & 0,059 \\
Einjährige Blühmischung & $-0,002$ & 0,553 & 0,275 & $-0,254$ & 0,591 & 0,168 \\
Amarant & $-0,002$ & 0,636 & 0,317 & $-0,254$ & 0,527 & 0,136 \\
Mais & $-0,002$ & $-0,135$ & $-0,069$ & $-0,254$ & 0,073 & $-0,091$ \\
Gemenge Amarant/Mais & $-0,002$ & 0,255 & 0,127 & $-0,254$ & 0,121 & $-0,067$ \\
Deutsches Weidelgras & n.v. & n.v. & n.v. & 0,372 & 0,786 & 0,579 \\
Mehrjährige Blühmischung & $-0,002$ & 0,377 & 0,187 & 0,406 & 0,594 & 0,500 \\
Durchwachsene Silphie & n.v. & n.v. & n.v. & 0,335 & 0,399 & 0,367 \\
\hline n.v. = Werte nicht verfügbar. & & & & & &
\end{tabular}




\section{Danksagung}

Mein besonderer Dank gilt Herrn Prof. Dr. Rolf Rauber, in dessen Händen die Betreuung dieser Dissertation lag. Vielen Dank für manche Anregung während der wissenschaftlichen Begleitung dieses Forschungsprojektes und für die vertrauensvolle und hilfreiche Unterstützung beim Verfassen der Dissertation.

Danken möchte ich auch Herrn Prof. Dr. Klaus Dittert für die Übernahme des Korreferats.

Gleichfalls gilt mein Dank allen Personen, die in dieses Projekt involviert waren: Besonders danke ich Herrn Thomas Seibold und Frau Christiane Münter für die großartige Durchführung der Feldversuche, Frau Gabi Kolle und Frau Kerstin Jespersen für die Durchführung der Laborarbeiten aber auch dem Feldpersonal für die Unterstützung bei der Datenerhebung. Herrn Dr. Benedikt Sauer und der Arbeitsgruppe der Abteilung Sedimentologie und Umweltgeochemie danke ich für die gute, konstruktive und freundschaftliche Zusammenarbeit im Verbundprojekt „Spurenelemente durch Energiepflanzen - Stoffströme und Handlungsempfehlungen für eine optimierte Prozessbiologie in Biogasanlagen“.

Nicht zustande gekommen wäre dieses Projekt ohne die finanzielle Förderung durch die Fachagentur Nachwachsende Rohstoffe e.V., Gülzow als Projektträger des Bundesministeriums für Ernährung und Landwirtschaft. Dafür gebührt ebenfalls mein großer Dank.

Nicht zuletzt geht mein Dank für Ihr Verständnis an meine Familie. Ihr habt mir während dieser aufwendigen Arbeit uneingeschränkt den Rücken freigehalten und rücksichtsvoll jegliche Entbehrungen und Anstrengungen mitgetragen. 